Ana B. Varas de la Fuente

\title{
PROGRAMAS DE REHABILITACIÓN RESPIRATORIA EN PACIENTES CON ENFERMEDAD PULMONAR OBSTRUCTIVA CRÓNICA
}

EFICACIA DE DISTINTAS MODALIDADES DE ENTRENAMIENTO EN EL MANTENIMIENTO DE LOS BENEFICIOS A LARGO PLAZO Y LA MODIFICACIÓN DEL NIVEL DE ACTIVIDAD FÍSICA

TESIS DOCTORAL

$2012 \cdot 2017$

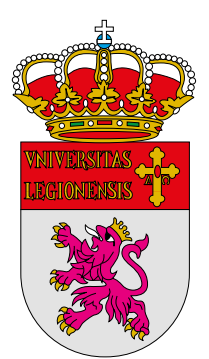

universidad deleón

Departamento de Ciencias Biomédicas

Programa de Doctorado en Ciencias de la Salud 


\section{ANA BEATRIZ VARAS DE LA FUENTE}

Graduada en Fisioterapia por la Universidad Autónoma de Madrid.

Máster en Fisioterapia Respiratoria y Cardiaca por la Universidad Autónoma de Madrid.

Máster en Fisioterapia Manual del Aparato Locomotor por la Universidad de Alcalá.

Profesora Titular de Fisioterapia Respiratoria y Cardiovascular. Escuela Universitaria de Fisioterapia de la ONC. Universidad Autónoma de Madrid.

Directores:

JULIÁN JOSÉ SUSPERREGUI LESACA

Doctor en Ciencias Matemáticas por la Universidad de Valladolid.

Profesor Titular de Universidad del Área de Matemática Aplicada.

Departamento de Matemáticas. Universidad de León.

JORDI VILARÓ CASAMITJANA

Doctor en Ciencias de la Salud y de la Vida por la Universidad Pompeeu Fabra.

Vicedecano de Investigación, Postgrado y Relaciones Internacionales.

Facultad de Ciencias de la Salud, Blanquerna. Universidad Ramón Llull. 
Índice

Agradecimientos 6

Presentación

PARTE I. MARCO TEÓRICO Y OBJETIVOS DE LA TESIS 8 MARCO TEÓRICO

1. La Enfermedad Pulmonar Obstructiva Crónica.

Mucho más que un problema respiratorio 8

1.1. Definición y etiopatogenia 8

1.2 Prevalencia e impacto de la enfermedad 9

1.3 Características clínicas e historia natural de la EPOC 10

1.4 La disfunción muscular periférica como factor de influencia en la limitación al ejercicio de los pacientes EPOC

2. La Rehabilitación Respiratoria en los pacientes con EPOC.

Un elemento básico en el abordaje terapéutico

2.1. Consideraciones generales acerca de la Rehabilitación

Respiratoria en pacientes con EPOC

2.2. Prevalencia de la Rehabilitación Respiratoria

2.3. Los programas de Rehabilitación Respiratoria autoadministrados

3. El mantenimiento de los beneficios de la Rehabilitación Respiratoria a largo plazo. Un reto por alcanzar

3.1. La duración de los efectos tras la Rehabilitación Respiratoria y el deterioro funcional de los pacientes con EPOC

3.2. Las intervenciones de mantenimiento tras los programas ambbulatorios de Rehabilitación Respiratoria

3.3. El mantenimiento de los efectos tras la Rehabilitación

Respiratoria autoadministrada 20

4. Actividad física y EPOC. Un problema por resolver

4.1. Actividad física: algunos datos generales

4.2. Nivel de actividad física en los pacientes EPOC

4.3. Factores que influyen en el bajo nivel de actividad física en los pacientes con EPOC

4.4. Efectos de la inactividad en la EPOC

4.5. Estrategias para aumentar el nivel de actividad física

en los pacientes EPOC

5. Los podómetros. Una herramienta útil en el entrenamiento y la modificación del nivel de actividad

5.1. El empleo de podómetros en diversos grupos poblacionales

5.2. El empleo de podómetros en la EPOC 


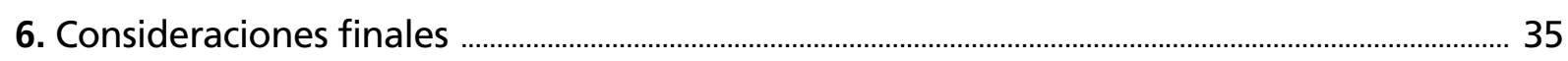

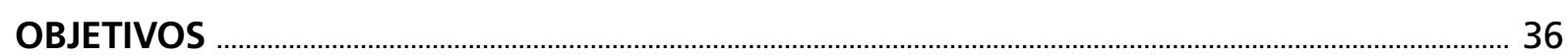

PARTE II. ESTUDIO 1: EFECTOS A CORTO Y LARGO PLAZO

DE TRES MODALIDADES DE PROGRAMAS CLÍNICOS

DE REHABILITACIÓN RESPIRATORIA EN PACIENTES EPOC ……………….................................. 39

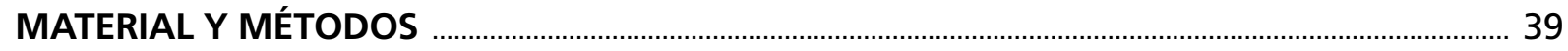

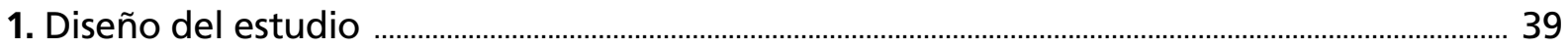

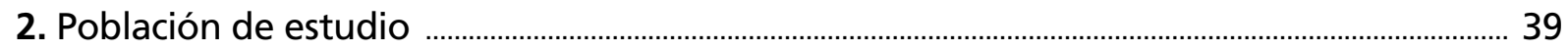

3. Muestreo y cálculo del tamaño muestral .................................................................................................. 40

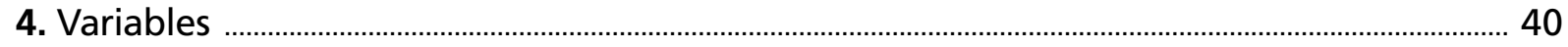

5. Intervención: Programas de Rehabilitación Respiratoria aplicados ............................................... 43

6. Almacenamiento y análisis de los datos ............................................................................................ 50

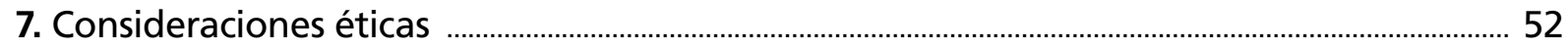

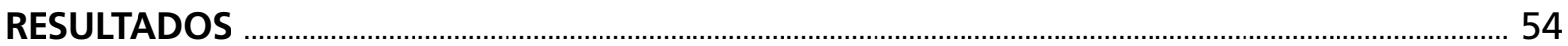

1. Características de la población de estudio ……………………………………………………………...... 54

2. Descripción de los resultados obtenidos en la muestra completa y segmentada según el tipo de entrenamiento ............................................................................................... 54

2.1. Resultados en la muestra completa ....................................................................................... 54

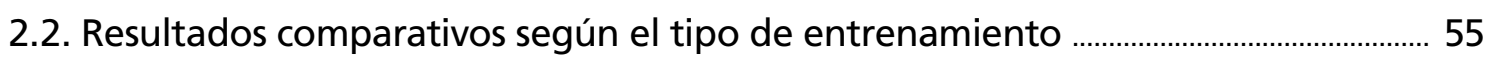

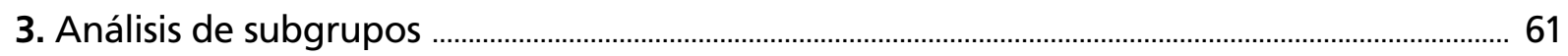

4. Factores de influencia sobre el deterioro en la tolerancia al ejercicio

tras el período de seguimiento ....................................................................................................................... 65

PARTE III. ESTUDIO 2: EFICACIA DE UN PROGRAMA

DE ENTRENAMIENTO AUTOADMINISTRADO E INCREMENTO

DEL NIVEL DE ACTIVIDAD GUIADO MEDIANTE PODÓMETRO EN PACIENTES CON EPOC

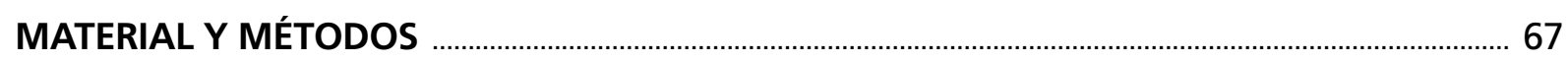

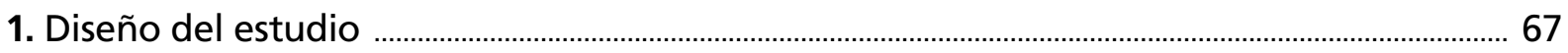

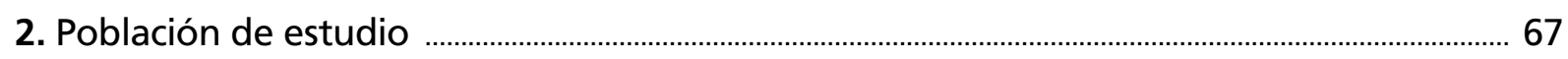

3. Muestreo y cálculo del tamaño muestral ........................................................................................... 67

4. Asignación de los sujetos a los grupos de estudio ............................................................................. 68

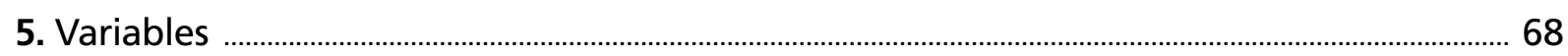

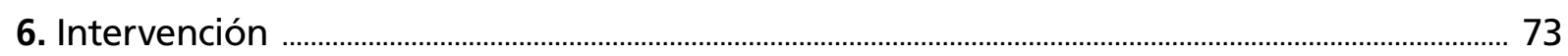

7. Almacenamiento de los datos …………………................................................................................. 79

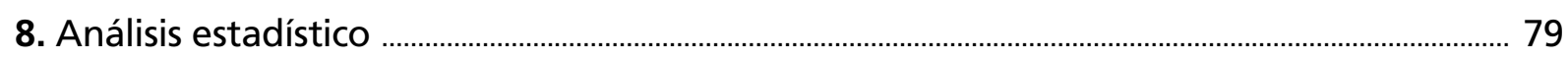

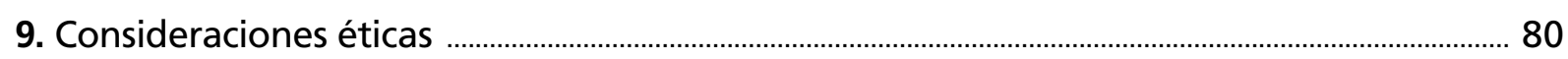

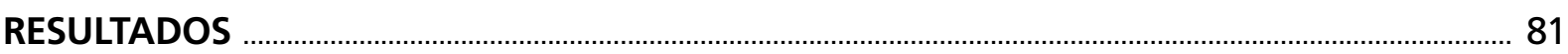




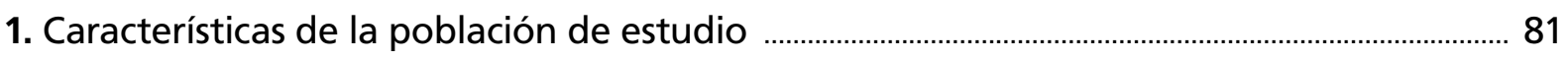

2. Efectos de las intervenciones a corto, medio y largo plazo ............................................................. 81

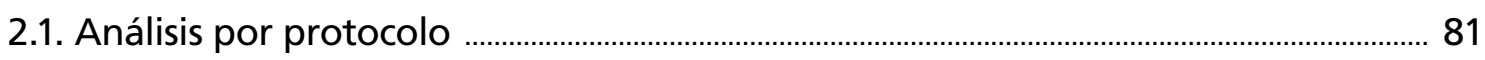

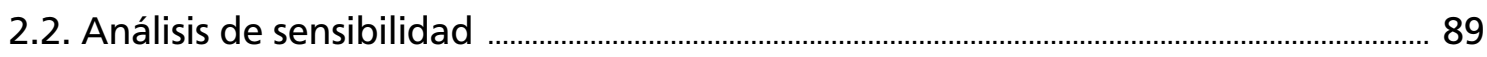

3. Diferencias en los resultados según distintos factores .................................................................. 92

4. Asociación entre la modificación en el nivel de actividad física y la tolerancia al ejercicio, la calidad de vida, la disnea basal y las exacerbaciones

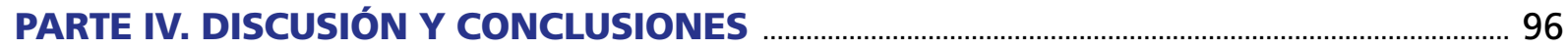

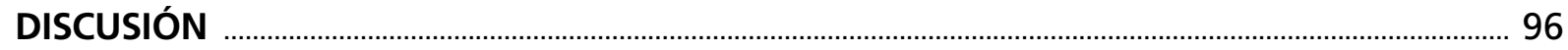

1. Efectos de la aplicación de programas ambulatorios de Rehabilitación

Respiratoria: Su duración y los factores influyentes ..................................................................................... 96

2. Eficacia del entrenamiento domiciliario en los pacientes EPOC ................................................. 98

3. La modificación del nivel de actividad física en la EPOC y su influencia sobre el estado funcional de los pacientes ................................................................. 102

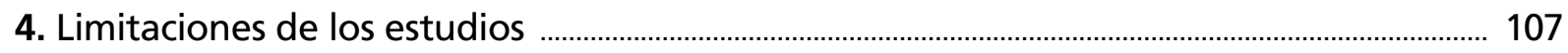

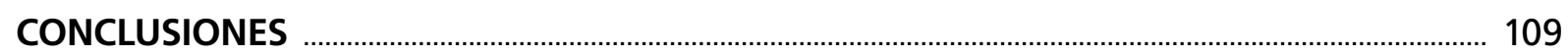

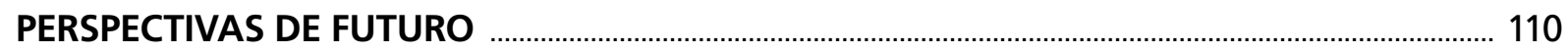

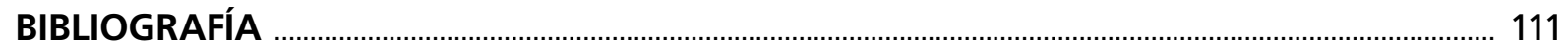

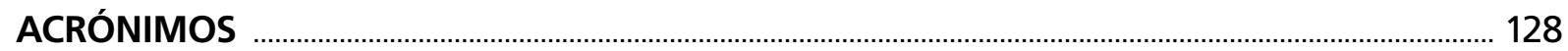

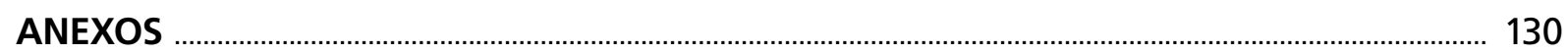

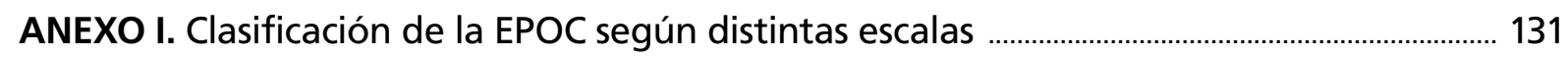

ANEXO II. Hoja informativa sobre la realización de Programas

de Rehabilitación Respiratoria en la Escuela Universitaria

de Fisioterapia de la ONCE y declaración de consentimiento

ANEXO III. Consentimiento informado para la utilización de datos personales vinculados a estudios de investigación en la Escuela Universitaria de Fisioterapia de la ONCE

ANEXO IV. Hoja de información y documento de consentimiento

del estudio: Eficacia de un programa de entrenamiento

autoadministrado e incremento del nivel de actividad guiado

mediante podómetro en pacientes con EPOC

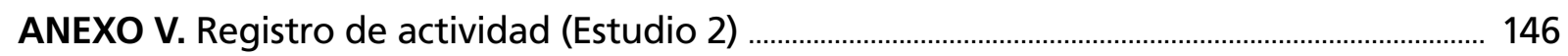

ANEXO VI. Hoja de recogida de datos - Entrevista inicial (Estudio 2) ........................................ 149

ANEXO VII. Hoja de recogida de variables (Estudio 2) ................................................................. 152 


\section{AGRADECIMIENTOS}

A mis directores de tesis, el Dr. Jordi Vilaró Casamitjana y el Dr. Julián José Susperregui Lesaca, por acompañarme en este largo proceso, ofreciéndome su ayuda desinteresada. Sus aportaciones han sido claves para la culminación del proyecto.

A la Escuela Universitaria de Fisioterapia de la ONCE, como institución que me ha ofrecido la infraestructura para llevar a cabo mi investigación. Así como al personal de administración y servicios del Centro, por su colaboración directa e indirecta.

A mis compañeras, Silvia Córdoba, Rocío Rueda e Irene Rodríguez, por su participación en la evaluación y tratamiento de los pacientes objeto de estudio. Quiero destacar su profesionalidad y agradecer especialmente su apoyo personal en los momentos complicados.

A José Luis Valero, responsable de Biblioteca en la Escuela Universitaria de Fisioterapia de la ONCE, por haberme atendido siempre con paciencia y buen carácter, a pesar de las circunstancias.

A mi familia y amigos, por estar ahí cuando se les necesita.

Y sobre todo, a Néstor, mi marido, por su comprensión, respeto y apoyo incondicional hacia mis decisiones profesionales. Su soporte me ha dado fuerzas y sus consejos me han aportado serenidad. Espero recuperar el tiempo que le he robado para dedicarlo a este proyecto. 


\section{PRESENTACIÓN}

El planteamiento temático de esta tesis tiene su origen en la experiencia clínica de más de diez años en el campo de la Fisioterapia Respiratoria y la Rehabilitación Respiratoria, fundamentalmente en la atención sanitaria de pacientes respiratorios crónicos adultos.

Durante todo este tiempo he participado en el diseño y la aplicación de distintos programas terapéuticos basados siempre en tres pilares fundamentales, la educación al paciente, la realización de técnicas específicas de Fisioterapia Respiratoria y el entrenamiento físico. Estos programas, que se efectuaban en la Escuela Universitaria de Fisioterapia de la ONCE (Madrid), han sido eficaces para mejorar el estado y la capacidad funcional de los pacientes. Sin embargo, la baja adhesión a las recomendaciones y pautas aportadas, así como la pérdida de los beneficios a medio-largo plazo fue la tónica general, tal y como pudimos comprobar en los pacientes que acudían nuevamente al Centro por cualquier motivo.

Estas observaciones empíricas apoyan las conclusiones de publicaciones que han ido surgiendo sobre este tema. En la Enfermedad Pulmonar Obstructiva Crónica (EPOC), ya contamos con una importante y fuerte evidencia de la efectividad de la Rehabilitación Respiratoria en general y el entrenamiento físico en particular, pero en los últimos años, la preocupación se ha centrado en el mantenimiento de estos efectos y la adhesión al ejercicio de nuestros pacientes.

Por otro lado, sabemos del alto porcentaje de pacientes EPOC que no tienen acceso a un programa ambulatorio en un centro sanitario, bien por la situación del propio paciente, bien por la insuficiente cobertura del sistema asistencial. Esto añade la necesidad de desarrollar programas eficaces que reduzcan la presencialidad en los centros, es decir, que acerquen la Rehabilitación Respiratoria a la comunidad.

En definitiva, hay que apostar por intervenciones que tiendan a dar autonomía al paciente y fomenten su implicación, que consigan una amplia duración de los efectos y alcancen una modificación de los hábitos para conseguir un estilo de vida más saludable.

Bajo esta motivación se desarrolla la línea argumental de esta tesis, en la que partiendo de una revisión de la literatura que se plasma en el marco teórico, se incluyen dos investigaciones: 1) Un estudio observacional que evalúa la duración de los efectos en diversos programas de Rehabilitación Respiratoria aplicados en nuestro Centro, así como la influencia de diversos factores en el mantenimiento. 2) Un ensayo clínico aleatorizado en el que se evalúa un programa de entrenamiento autoadministrado asociado a una estrategia de cambio de comportamiento en pacientes EPOC sedentarios. En la parte final dela tesis se incorpora un apartado de discusión y conclusiones comunes a ambos estudios. 


\section{PARTE I \\ MARCO TEÓRICO Y OBJETIVOS DE LA TESIS}

\section{MARCO TEÓRICO}

\section{La Enfermedad Pulmonar Obstructiva Crónica. Mucho más que un problema respiratorio}

\subsection{Definición y etiopatogenia}

Según la última actualización de la Global Iniciative for Lung Ostructive Disease (GOLD) de 2016, la EPOC se define como "A common preventable and treatable disease, is characterized by persistent airflow limitation that is usually progressive and associated with an enhanced chronic inflammatory response in the airways and the lung to noxious particles or gases. Exacerbations and comorbidities contribute to the overall severity in individual patients" (1).

Por su parte, la Guía Española para el Manejo de la EPOC (GesEPOC) de 2012, establece la siguiente definición: “La EPOC se define como una enfermedad respiratoria caracterizada esencialmente por una limitación crónica al flujo aéreo que no es totalmente reversible. Esta limitación al flujo aéreo se suele manifestar en forma de disnea y, por lo general, es progresiva. La limitación al flujo aéreo se asocia a una respuesta inflamatoria anormal de los pulmones a partículas nocivas y gases, principalmente derivados del humo de tabaco, que pueden producir otros síntomas como tos crónica, acompañada o no de expectoración. La EPOC se caracteriza también por la presencia de agudizaciones y por la frecuente presencia de comorbilidades que pueden contribuir a la gravedad en algunos pacientes" (2). Esta definición está de acuerdo y extiende las propuestas por la American Thoracic Society (ATS) y la European Respiratory Society (ERS) (3).

La principal causa para el desarrollo de la enfermedad es la inhalación de humo de tabaco (4). Varios estudios de cohortes han demostrado la asociación entre tabaquismo y obstrucción bronquial (con un declive acelerado del volumen espiratorio forzado en el primer segundo $\left.\left(\mathrm{FEV}_{1}\right)\right)(5,6)$ y se estima que el riesgo de desarrollar EPOC en fumadores activos es del $25 \%(7)$. Del mismo modo, la exposición pasiva al humo del tabaco también aumenta la incidenncia de la EPOC, según demostró un estudio de cohortes realizado sobre más de 6.000 mujeres chinas mayores de 50 años (8).

Sin embargo, no todos los individuos fumadores presentan EPOC, por lo que se piensa que el desarrollo de la enfermedad está condicionado por el estilo de vida de los sujetos y la concurrencia de otros factores (1).

En relación a otros determinantes, aún son necesarios más estudios, ya que los actuales se basan en cohortes de larga duración, pero que en ningún caso abarcan el curso evolutivo total de la enfermedad y no analizan las condiciones prenatales y peri-natales (1). No obstante, podemos destacar:

- Factores genéticos. No se ha determinado un genotipo específico, excepto el déficit de alfa-1-antitripsina, pero sí una mayor susceptibilidad en pacientes con antecedentes familiares de la enfermedad (9). 
- El sexo. La prevalencia de la EPOC es mayor en varones, sin embargo, las diferencias se están reduciendo a medida que el consumo de tabaco por parte de las mujeres aumenta (9). De hecho, se considera que las mujeres presentan un mayor riesgo de desarrollo precoz de la enfermedad ante exposiciones menores al tabaco y un más rápido declive en la función pulmonar (10).

- El desarrollo del aparato respiratorio en la etapa embrionaria y en la infancia. Se ha demostrado una relación entre el bajo peso al nacer (11) y unas peores condiciones de vida durante la infancia (12) con un declive prematuro en la función pulmonar.

- Las infecciones respiratorias de repetición en la infancia. Aumentan el riesgo de deterioro precoz en la función pulmonar en la edad adulta y por tanto, de desarrollar EPOC (13).

- La inhalación de humos procedentes de la combustión de biomasa también constituye un factor relevante en el desarrollo de la EPOC, independiente del sexo o del consumo tabáquico (14).

- La exposición laboral a polvos, humos o gases tóxicos contribuye también al desarrollo de la EPOC en pacientes susceptibles (15) y a una peor evolución de la enfermedad en pacientes ya diagnosticados (16).

- La contaminación ambiental no se ha demostrado como agente causal, pero sí un factor influyente en el empeoramiento de los síntomas (15).

- Factores socioeconómicos. Existe una relación inversa entre el nivel socioeconómico y el riesgo de desarrollar EPOC, independientemente del sexo y el hábito tabáquico (17).

- El asma y los antecedentes familiares de asma son factores predisponentes para el desarrollo de la EPOC, independientes del tabaco $(13,18)$.

Al margen de la causa o factor predisponente, el mecanismo fisiopatológico de la EPOC se fundamenta en la presencia de una reacción inflamatoria en el pulmón (vías aéreas y parénquima) acompañada de cambios estructurales, consecuencia de los procesos de destrucción y reparación tisular. Estos procesos se ven potenciados por el incremento del estrés oxidativo observado en estos pacientes y por un disbalance proteasas-antiproteasas en el pulmón (1).

Por tanto, la limitación crónica al flujo aéreo de los pacientes EPOC se produce como consecuencia de una mezcla de afectación en las vías aéreas de pequeño calibre (bronquiolitis) y la destrucción del parénquima (enfisema). El estrechamiento en las vías aéreas provocado por los procesos inflamatorios y los cambios estructurales, junto con la pérdida de tracción radial del tejido alveolar circundante, son los mecanismos que justifican la obstrucción al flujo aéreo espiratorio en estos pacientes (1).

\subsection{Prevalencia e impacto de la enfermedad}

La EPOC constituye una de las enfermedades que causan mayor morbimortalidad en la población adulta. Según el Global Burden of Disease Study de 2010 (19), el número de casos diagnosticados en el mundo ha pasado de 210 millones en 1996 a casi 329 millones en 2010 . Su prevalencia en 1996 era del $10 \%$ (11,8\% en varones y 5,8\% en mujeres) (20) y según una revisión realizada hasta 2004, ésta se mantenía entre el 9-10\%, si bien existía una gran variabilidad entre los países (21). 
En una revisión sobre datos epidemiológicos en población europea hasta 2010, se encontró una gran diferencia en la prevalencia de la EPOC entre los distintos países (del 2,1\% al 26,1\%), observándose la necesidad de establecer criterios comunes de diagnóstico y clasificación de la enfermedad, así como de estrategias similares de registro epidemiológico (22).

En España, según el Estudio EPI-SCAN, su prevalencia en 2007 era del 10,2\% (15,1\% en varones y $5,7 \%$ en mujeres), alcanzándose cifras de más de 2 millones de pacientes diagnosticados (23). No obstante, se considera que existe un alto nivel de infradiagnóstico (en torno al 73\%), por lo que podría haber más de 1,5 millones de españoles sin diagnosticar y por tanto, sin tratamiento (24).

La mortalidad por EPOC en el mundo se está reduciendo ligeramente. Atendiendo a tasas de mortalidad corregidas por edad, ésta ha pasado de 18,5 muertes por cada 100.000 habitantes en 2006 a 15,9 por 100.000 en 2013 (25). Sin embargo, como causa de muerte está tomando mayor relevancia. Según la Organización Mundial de la Salud (OMS), la EPOC causó más de 3 millones de fallecimientos en el mundo en el 2005 y pasará de ser la quinta causa de muerte en 2002 a convertirse en la tercera en 2030 (25). Incluso, algunos estudios ya han determinado que esta enfermedad ha pasado a ser la tercera causa de muerte en el mundo en el $2010(19,26)$.

En Europa también hay una gran variabilidad entre los países en cuanto a la tasa de mortalidad por EPOC, oscilando entre 7,6 y 36,1 fallecimientos por cada 100.000 habitantes (22). En España, según datos de 2013, constituye la cuarta causa de muerte después del cáncer, las enfermedades cardiacas y las lesiones cerebrovasculares (27). En 2014 la tasa de mortalidad fue de 18,9/100.000 habitantes (28).

Por otro lado, cabe indicar que la EPOC supone un alto coste económico para las administraciones sanitarias. Según la ERS, en un documento de consenso publicado en 2003 (29), las enfermedades respiratorias constituyen el $6 \%$ del gasto sanitario total en Europa, sobre todo a consecuencia de la EPOC ( $56 \%$ del coste total de las enfermedades respiratorias), lo que se traduce en alrededor de 38,9 billones de euros al año entre gastos directos e indirectos.

En España, según la "Estrategia en EPOC" del Sistema Nacional de Salud publicada por el Ministerio de Sanidad y Consumo en 2009 (30), los costes de esta enfermedad se estiman entre 750 y 1.000 millones de euros al año (entre 1.712 y 3.238 euros/año por paciente).

\subsection{Características clínicas e historia natural de la EPOC}

La EPOC es una enfermedad compleja, multicomponente, crónica y progresiva (2), lo que hace necesario establecer una clasificación clínica de la enfermedad basada en fenotipos (31). La Guía GesEPOC describe cuatro fenotipos clínicos: No agudizador, mixto EPOC-asma, agudizador con enfisema y agudizador con bronquitis crónica (2).

La historia natural de la EPOC conduce a un declive progresivo del FEV ${ }_{1}$, fundamentalmente mientras se mantenga el hábito tabáquico (6), si bien, esta evolución es heterogénea, observándose en muchos pacientes, estabilidad en su función pulmonar durante varios años $(32,33)$. La obstrucción al flujo aéreo espiratorio conduce a una hiperinsuflación con limitación de la capacidad inspiratoria, especialmente durante el esfuerzo físico (hiperinsuflación dinámica) (34). Así mismo, la hipersecreción mucosa que presentan muchos pacientes con EPOC aumenta la incidencia de exacerbaciones que a su vez, constituyen un factor relevante en el deterioro acelerado del FEV 1 (35). 
Por otro lado, el componente extrapulmonar de la EPOC ha sido descrito ampliamente, lo que conduce a definir a la EPOC como una enfermedad sistémica $(36,37)$. La caquexia, la disfunción muscular periférica y las alteraciones del sistema nervioso como la depresión y la ansiedad, son las implicaciones sistémicas más características y cuya presencia puede estar asociada a la inflamación sistémica, la hipoxia tisular, el estrés oxidativo aumentado y la inactividad (37). La inflamación sistémica también puede producir síndrome metabólico (38).

Además, la presencia de comorbilidades asociadas es altamente frecuente (39). En un estudio observacional realizado sobre más de 200 pacientes EPOC se encontró una prevalencia de comorbilidades del 97\% (41) y en otro, realizado en la Comunidad de Madrid, el porcentaje era del $90 \%$, con una media de dos comorbilidades por paciente (42). Entre las enfermedades asociadas más frecuentes se encuentran las cardiovasculares (hipertensión arterial, cardiopatía isquémica, tromboembolismo), la diabetes, la insuficiencia renal, el cáncer de pulmón, la osteoporosis, la obesidad y el síndrome de apnea del sueño (34).

Toda esta contextualización de la EPOC como enfermedad multifactorial ha hecho que la clasificación de la gravedad de la enfermedad según el $\mathrm{FEV}_{1}(\%)$ descrita por la Iniciativa GOLD (43), resultara incompleta como factor pronóstico. De este modo, se han desarrollado índices multicomponente como el Body Mass Index, Obstruction, Dyspnea and Execercise (BODE) (44), el Body Mass Index. Obstruction, Dyspnea and Exacerbations (BODEX) (45) e incluso, la nueva versión de la clasificación GOLD de 2011 en cuatro grupos( 46) (en el Anexo I se muestran estas escalas de clasificación).

\subsection{La disfunción muscular periférica como factor de influencia en la limitación al ejercicio de los pacientes EPOC}

La disfunción muscular periférica es una de las principales consecuencias sistémicas de la EPOC, considerándose uno de los factores fundamentales de influencia en la intolerancia al ejercicio de estos pacientes (47-49). Además, puede relacionarse con una peor calidad de vida (49), una mayor mortalidad $(49,50)$ y morbilidad, al alterar la realización de actividades cotidianas (49).

Se ha descrito la presencia de diferentes factores en el desarrollo de la disfunción muscular periférica en la EPOC (Figura 1):

- Atrofia muscular. Los pacientes EPOC sufren un proceso de atrofia muscular que se manifiesta por un menor índice de masa magra que los individuos sanos de la misma edad, incluso manteniendo el índice de masa corporal (IMC), que afecta fundamentalmente a los miembros inferiores (MMII) (51).

- Cambios estructurales en el músculo. En los pacientes EPOC se produce una transformación de fibras tipo I en fibras tipo Ilx (52-54), observándose una proporción de fibras tipo I inversamente proporcional a la severidad de la enfermedad (52). Así mismo, presentan menor densidad capilar en la fibra muscular periférica (54).

- Cambios en la función mitocondrial y en la bioenergética muscular. En los pacientes EPOC se produce una reducción en la densidad (55) y alteraciones en la función mitocondrial de la fibra muscular $(56,57)$, así como cambios en la bioenergética, con un disbalance entre metabolismo oxidativo y glicolítico (58).

A pesar de la amplia investigación en este campo, aún no se conocen exactamente los mecanismos que dan lugar a la disfunción muscular periférica en la EPOC. Un debate habitual es la 
inactividad como agente causal frente a la presencia de miopatías específicas, si bien, parece que es más probable la influencia del desuso (49), puesto que los cambios en la musculatura en la EPOC son similares a los que se producen tras un período de inactividad y además, esta disfunción responde positivamente al entrenamiento. La Actualización sobre Disfunción Muscular Periférica en EPOC publicada en 2014 por la ATS/ERS (49) afirma que otras causas están implicadas, tales como:

- La inflamación sistémica que genera proteólisis y atrofia muscular (59).

- El estrés oxidativo en la fibra muscular que conduce a oxidación de las proteínas contráctiles (60), y que está aumentado en los pacientes EPOC en reposo y tras el ejercicio $(61,62)$, especialmente en aquellos con hipoxia crónica $(62,63)$.

Figura 1. Cambios morfológicos y estructurales en la musculatura de los miembros inferiores en pacientes EPOC.

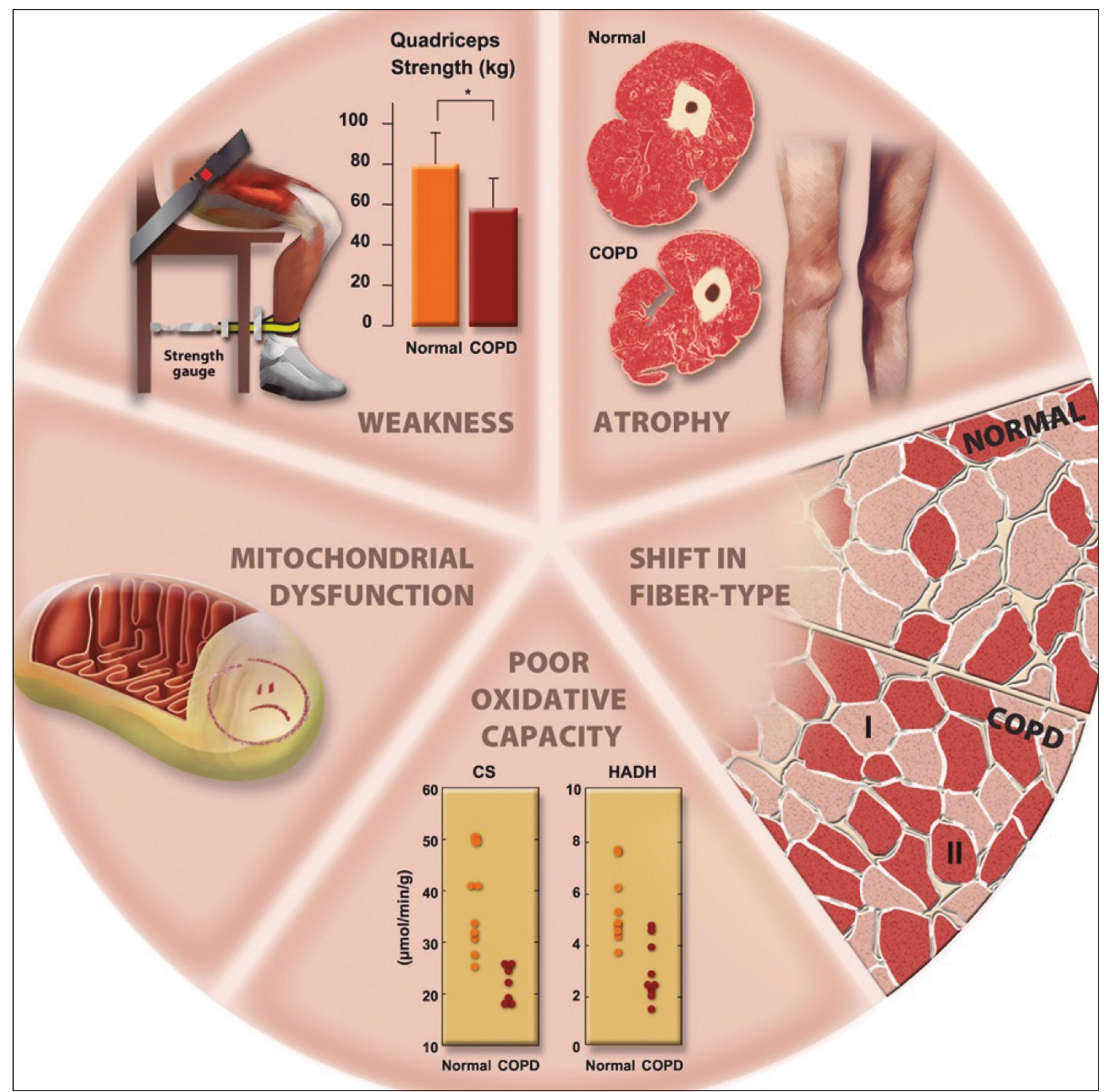

Figura extraída de la Actualización sobre Disfunción Muscular Periférica en EPOC publicada por la ATS/ERS en 2014 (49).

CS: Citrato sintetasa.

HDHA: 3-Hdroxacil CoA Deshidrogenasa. 
- El consumo de corticoides, ya que éstos generan una inhibición de la síntesis proteica

y un incremento de los mecanismos de proteólisis en la fibra muscular (64).

Fundamentalmente, la disfunción muscular periférica en la EPOC se ha analizado en el cuádriceps, objetivándose una reducción en la fuerza contráctil en el 20-30\% de los pacientes (49), evaluada tanto de forma activa (65) como mediante la estimulación magnética del nervio femoral (66), con un declive progresivo de ésta, independiente de factores como la función pulmonar y las exacerbaciones (67).

Por otro lado, también se ha objetivado una reducción en la resistencia muscular, fundamentalmente del cuádriceps, dando lugar a fatiga, mientras esto no se ha descrito para la musculatura de los miembros superiores (MMSS) (49). La reducción en la resistencia muscular del cuádriceps se ha encontrado incluso en EPOC leves-moderados sin pérdida ni en la masa magra ni en la fuerza, con una correlación inversa y significativa entre resistencia muscular y estrés oxidativo (68) e hipoxia (60).

Si bien la hiperinsuflación dinámica que desarrollan los individuos EPOC durante el ejercicio limita la capacidad de esfuerzo (34), no puede afirmarse que sea el factor principal. De hecho, la capacidad de ejercicio no mejora significativamente tras la broncodilatación (63), lo que pone de manifiesto que la función pulmonar no es el principal limitante. Por su parte, la mayor fatigabilidad producida por la disfunción muscular tiene una implicación más relevante $(69,70)$. Además, un efecto de la disfunción muscular periférica es la producción precoz de lactato durante el ejercicio, lo que contribuye al incremento en las demandas ventilatorias y a la aparición de disnea (71) que constituye un factor adicional e indirecto a la reducción en la capacidad de ejercicio.

\section{La Rehabilitación Respiratoria en los pacientes con EPOC. Un elemento básico en el abordaje terapéutico}

\subsection{Consideraciones generales acerca de la Rehabilitación Respiratoria en los pacientes con EPOC}

La Rehabilitación Respiratoria (RR) constituye una alternativa terapéutica muy interesante en el abordaje integral de los pacientes EPOC, orientada a aumentar la tolerancia al ejercicio a través de optimizar su función muscular. La ATS y la ERS la definen del siguiente modo: "Pulmonary rehabilitation is a comprehensive intervention based on a thorough patient assessment followed by patienttailored therapies that include, but are not limited to, exercise training, education, and behavior change, designed to improve the physical and psychological condition of people with chronic respiratory disease and to promote the long-term adherence to health-enhancing behaviors" (72).

La RR mejora la tolerancia al ejercicio, la disnea y la calidad de vida (73-75), así como la capacidad funcional (76). También se ha demostrado que reduce el número de exacerbaciones $(73,74)$, lo que conduce a una disminución en el consumo de recursos sanitarios (47). Además, algunos estudios muestran que programas adaptados de RR aplicados de forma temprana tras una exacerbación, reducen la mortalidad y las recidivas (77), por lo que se recomienda el inicio inmediato de la RR tras una exacerbación $(78,79)$. No obstante, en la última Revisión Cochrane sobre el tema se concluye que la evidencia del efecto de la RR tras exacerbación en EPOC sobre los reingresos y la mortalidad es modesta y heterogénea, y son necesarios más estudios al respecto (80). 
Actualmente, todas las guías de manejo de la EPOC, incluyendo la Iniciativa GOLD desde su versión de 2001(43) hasta su última versión de 2016 (1), la Sociedad Española de Neumología y Cirugía Torácica (SEPAR) $(48,81,82)$, la ATS y la ERS $(47,49,72)$ han determinado la eficacia de estos programas en la disminución de la disnea, la mejoría en la capacidad de esfuerzo y en la calidad de vida, así como en la reducción de las exacerbaciones de los pacientes EPOC. Del mismo modo, la "Estrategia en EPOC" del Sistema Nacional de Salud de 2009 (30) recoge como objetivo la implementación de estos programas en los centros sanitarios del Sistema Nacional de Salud Español. Así mismo, la Guía GesEPOC también reconoce a la RR como un importante elemento en el tratamiento de los pacientes EPOC, tanto en su versión de 2012 como en la actualización de 2014 (2,34).

Entre los componentes de los programas de Rehabilitación Respiratoria (PRR), el entrenamiento físico es uno de los más importantes, gracias a los beneficios que reporta, con un nivel de evidencia 1++ y un grado de recomendación A en los pacientes EPOC (83). Se ha demostrado que el entrenamiento físico produce unos cambios morfológicos en la fibra muscular que contrarrestan los efectos de la disfunción muscular periférica (84). Tras un programa de entrenamiento, se observa un incremento en la sección transversal del cuádriceps (85), así como un remodelado muscular con transformación de fibras tipo II en tipo I y un incremento en la densidad capilar por fibra $(85,86)$.

Aunque el gold standard es el ejercicio aeróbico, la inclusión de trabajo de fuerza potencia sus beneficios (87). El entrenamiento de fuerza de miembros superiores no aumenta la capacidad aeróbica y realizado de forma aislada tampoco tiene impacto en disnea o calidad de vida, pero sí mejora la funcionalidad para las actividades de la vida diaria (72).

Según las guías de práctica clínica, $(49,81)$ la duración de los programas debe ser de al menos seis semanas, incluyendo entrenamiento aeróbico, mínimo tres días a la semana durante 20 minutos, a una intensidad igual o superior al $60 \%$ de su carga máxima de trabajo tolerada (Wmax) (72), puesto que intensidades menores no alcanzan adaptaciones cardiovasculares y musculares (88). Puede realizarse a carga constante o de forma interválica, no habiéndose evidenciado la mayor eficacia de una modalidad sobre la otra, si bien el entrenamiento interválico es más tolerable por los pacientes, especialmente por aquellos con mayor disnea asociada al ejercicio (72).

\subsection{Prevalencia de la Rehabilitación Respiratoria}

La RR, a pesar de sus múltiples beneficios, está infrautilizada y es inaccesible para muchos pacientes $(89,90)$. Se considera que en el mundo sólo entre el 3 y el $16 \%$ de los sujetos susceptibles de recibir esta terapia, son referidos a programas específicos, de los cuales sólo consiguen acceder entre un $1 \%$ y un $2 \%$, y lo terminan un 0,7\% (Figura 2) (90). En España, la implementación de estos programas es aún muy baja y desigual según las distintas comunidades autónomas $(91,92)$.

En un estudio reciente en el que se analizaron más de 400 programas de RR en todo el mundo (87\% en países europeos y Estados Unidos), también concluyeron que la implementación de la RR era desigual según los países, no sólo en cuanto a centros disponibles o pacientes tratados, sino a la constitución de los equipos, las características de los programas y los criterios utilizados para evaluar la calidad de los resultados (93).

Las causas fundamentales del bajo acceso de los pacientes EPOC a los programas de RR son los problemas de desplazamiento (94), la insuficiente inversión, la mala distribución de los 
Figura 2. Prevalencia de la Rehabilitación Respiratoria en la EPOC.

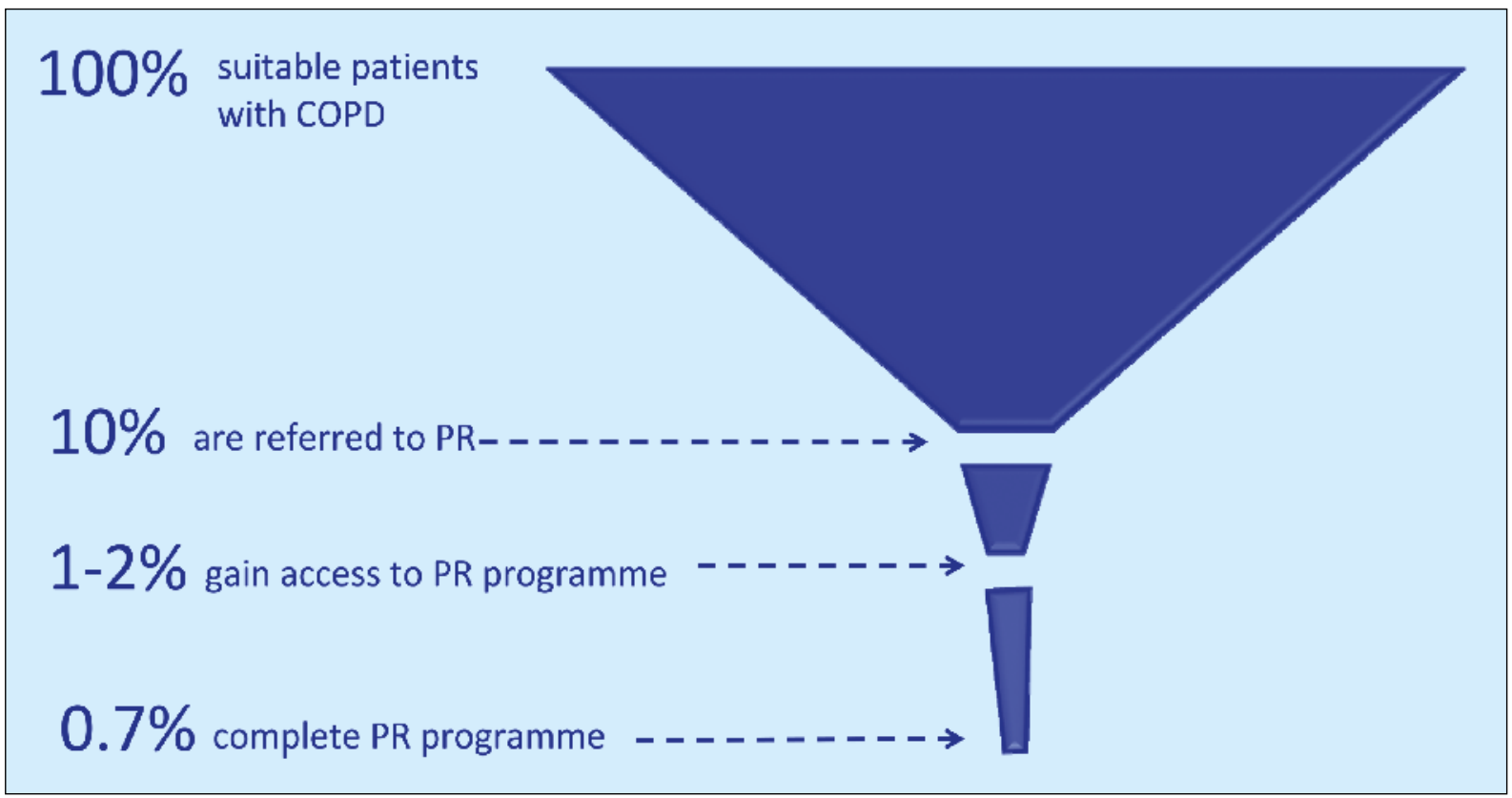

Figura extraída del estudio de Johnston y col., 2010 (90).

Representa el porcentaje de pacientes con EPOC a nivel mundial que son referidos a un PRR, acceden a él y lo finalizan.

centros sanitarios y el desconocimiento por parte de los pacientes, las entidades financiadoras y los propios profesionales sanitarios (89).

Por otro lado, Sabit y col. (95) determinaron las causas de abandono en los programas de $\mathrm{RR}$, hallando que la disnea basal, el número de exacerbaciones previas y la distancia de su domicilio al centro sanitario, eran factores independientes asociados con el incumplimiento de los pacientes. Por su parte, Fischer y col. (96) , en una investigación cualitativa realizada en 2007, identifican como causas de abandono en los programas, la alta intensidad del entrenamiento que conduce a fatiga posterior, la falta de beneficios percibidos por los pacientes, las dificultades de transporte, la incompatibilidad con otras actividades y la presencia de enfermedades asociada. Del mismo modo, una personalidad más escéptica parece más proclive al abandono (96). Así mismo, una revisión efectuada en 2012 coincide con las conclusiones de los estudios anteriores y añade otras causas relevantes como la duración de los programas y la insuficiente información aportada por los profesionales sanitarios sobre los beneficios del ejercicio (97).

\subsection{Los programas de Rehabilitación Respiratoria autoadministrados}

Una opción interesante para incrementar la cobertura de la RR son los programas autoadministrados o domiciliarios. Éstos requieren una menor infraestructura, ya que el paciente efectúa el entrenamiento en su domicilio o en la comunidad, siguiendo las pautas aportadas por el equipo sanitario, evitando así además, desplazamientos costosos. Sin embargo, es importante que estén bien planificados, que los pacientes se comprometan a efectuarlo, que sean adecuadamente adiestrados y que se lleve a cabo un seguimiento estricto.

Según una revisión que analizaba estudios publicados hasta 2009, la RR autoadministrada consigue beneficios similares en capacidad de ejercicio y calidad de vida a la modalidad ambulatoria, por lo que puede ser una alternativa útil para aquellos pacientes con dificultad de acceso a los programas ambulatorios (98). Por su parte, investigaciones efectuadas en los 
últimos 10 años corroboran esta afirmación $(99,100)$. Sin embargo, aún no hay datos concluyentes sobre su efecto en las exacerbaciones $(98,99)$.

Así mismo, al comparar la RR domiciliaria con un grupo control al que se le aplican los cuidados habituales, también se observa mejoría en la capacidad de ejercicio, la calidad de vida $(101-105)$, en reducir la disnea basal $(101,104,105)$ y los marcadores inflamatorios $(102)$.

Sin embargo, a pesar de estos beneficios, la implementación de programas autoadministrados es aún escasa $(89,93)$. La ATS y la ERS establecen como un objetivo prioritario, una mayor inversión en programas de este tipo, que permitan el acceso a un mayor número de pacientes (89).

\section{Características de los programas}

Los programas autoadministrados intentan replicar los programas ambulatorios, modificando el entorno y los mecanismos de control de los pacientes. De hecho, la mayoría de ellos tienen una duración entre 8 y 12 semanas, aunque se han encontrado algunos de hasta 16 semanas (99).

La mayor parte de los programas incluyen unas sesiones educativas previas en las que se aportan estrategias de autocuidados, control de la enfermedad, consejos nutricionales y sobre actividad física, técnicas de limpieza bronquial y de ahorro de energía,etc $(99,100,104,106,107)$. Así mismo, algunos programas contemplan el entrenamiento específico de músculos respiratorios $(101,107,108)$ y más habitualmente, ejercicios de fuerza de miembros $(99-101,103,104,106,107)$.

En cuanto al entrenamiento aeróbico, los estudios publicados en los últimos años describen varias modalidades:

- Entrenamiento en cicloergómetro, de forma similar a como se realizaría en el centro sanitario (101) o con intensidades ligeramente inferiores (100).

- Entrenamiento mediante marcha libre (102,103,105-109).

- Entrenamiento combinado cicloergómetro/marcha libre $(99,104)$.

El entrenamiento mediante caminata parece ser el más utilizado, dada su facilidad de aplicación fuera del contexto hospitalario, aunque las estrategias empleadas han sido diferentes, tanto en la duración/frecuencia como en la intensidad. Se han encontrado programas que pautaban caminar tres días por semana durante 40 minutos (103), cuatro días durante 20 minutos (105), cinco días por semana aumentando el tiempo de 15 a 45 minutos (107) e incluso, recomendación de caminar a diario durante un mínimo de 15 minutos (108) o el tiempo que pudieran $(102,106,109)$. En cuanto a la intensidad, se han descrito desde programas sin pautas concretas (106), basados en frecuencia cardiaca (caminar al $60-70 \%$ de la frecuencia cardiaca máxima teórica) (103), según la disnea percibida (108) o según una velocidad predeterminada, que podía ser fija (4 Km/h) (107) o basada en una prueba incremental previa $(102,105,109)$.

En los programas que empleaban la velocidad de marcha para establecer la intensidad del entrenamiento, se han utilizado distintos sistemas de feed-back para que los pacientes tuvieran conocimiento del nivel al que estaban entrenando. Desde el uso de podómetros para controlar la velocidad (107) hasta sistemas más complejos $(102,105,109)$. Liu y col. (102) diseñaron un programa musical con distintas melodías que diferían en el ritmo, correspondiendo cada una a diversas velocidades de marcha. Los pacientes debían entrenar escuchando y siguiendo el ritmo de la melodía que correspondía a su nivel de entrenamiento. Por otro lado, Pomidori y col. (105) utilizaron dos sistemas de feed-back, un programa sonoro que marcaba el ritmo 
y alcanzar una distancia en un tiempo, no encontrándose diferencias entre ambos sistemas, tras finalizar los programas.

En relación al control de los pacientes durante los programas domiciliarios, se han descrito varios mecanismos:

- Visita en domicilio periódica para verificar el cumplimiento de lo pautado, detectar problemas y establecer la progresión. Esas visitas pueden hacerse semanalmente $(99,101)$, cada dos semanas (104) o incluso, de forma sorpresiva (108).

- Revisión en el centro sanitario cada dos semanas (102) o cada cuatro semanas $(108,109)$.

- Control telefónico semanal $(100,101,103,104,106)$ o según necesidades (cuando se detectaba que los pacientes no cumplían el programa) $(102,109)$.

- Cumplimentación de un diario de actividad que debían reportar a los responsables sanitarios periódicamente para el seguimiento de la realización del programa y la detección de problemas $(101,103)$.

\section{El mantenimiento de los beneficios de la Rehabilitación Respiratoria a largo plazo. Un reto por alcanzar}

\subsection{La duración de los efectos tras la Rehabilitación Respiratoria y el deterioro funcional de los pacientes con EPOC}

Los efectos de la RR tienen una duración limitada $(47,81,110)$ influyendo tanto la evolución de la propia enfermedad (111) como las características del programa de RR llevado a cabo (duración e intensidad) (112). Se estima que los efectos tras la RR se pierden entre los 6 y los 12 mese (72), aunque programas de larga duración (seis meses de entrenamiento) pueden mantener sus efectos sobre la capacidad de ejercicio y la calidad de vida hasta 18 meses (113).

En una revisión sobre el mantenimiento de los efectos tras RR en pacientes respiratorios crónicos, que incluye 15 estudios sobre EPOC, concluye que independientemente del tipo de programa utilizado, se alcanzan beneficios en la capacidad de ejercicio, la calidad de vida y el consumo de recursos sanitarios, pero no hay evidencias suficientes sobre su permanencia a largo plazo. Los autores afirman que esto puede deberse a que los programas ambulatorios de RR no incluyen actuaciones dirigidas a modificar los hábitos de los pacientes (114).

La pérdida de los beneficios de la RR es especialmente importante en cuanto a tolerancia al esfuerzo, puesto que se sabe que la capacidad de ejercicio constituye un predictor de mortalidad, independiente de la edad, el IMC y el FEV . Esta asociación se ha evaluado fundamentalmente con la Prueba de Marcha de Seis Minutos (Six Minutes Walking Test, 6MWT), la cual tiene un alto valor predictivo de supervivencia (115-118), independiente de la función pulmonar (116).

Además, la capacidad de ejercicio de los pacientes EPOC decrece a lo largo del tiempo $(119,120)$, especialmente en los individuos más severos $(117,118)$ y de forma precoz desde el comienzo de los primeros síntomas (34). El deterioro en la capacidad de ejercicio se asocia al declive en la función pulmonar, pero no de forma proporcional, de tal modo que la pérdida

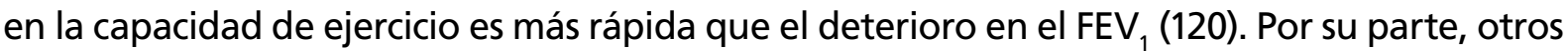
estudios describen su independencia con el FEV $(115,121)$.

Las exacerbaciones son un elemento a tener en cuenta, puesto que el incremento en la inflamación sistémica asociado a los episodios de reagudización, conduce a un deterioro en la 
capacidad de ejercicio posterior a cada episodio (122). Parece que la duración de los episodios de exacerbación tiene una asociación directa con la pérdida en la capacidad de ejercicio evaluada mediante el 6MWT, mayor incluso que la frecuencia de éstas (123). Así mismo, los ingresos hospitalarios por cualquier causa influyen en el deterioro funcional de estos pacientes (123).

\subsection{Las intervenciones de mantenimiento tras los programas ambulatorios de Rehabilitación Respiratoria}

Todo lo expuesto hace pensar que el empleo de intervenciones que redunden en una mayor capacidad de ejercicio de estos pacientes, permitirá alcanzar un pronóstico más favorable en la enfermedad. Por tanto, surge la necesidad de implementar estrategias de mantenimiento tras la RR (124), aunque actualmente no existen consensos al respecto y los estudios publicados en este campo no son muy abundantes (81). De hecho, en un documento de consenso de la ATS/ERS de 2015 sobre necesidades de investigación en la EPOC, se incluyen las estrategias de mantenimiento tras RR como una línea básica para investigaciones futuras (125).

En una revisión publicada por Troosters y col. en 2005 (112), se concluye que los programas de mantenimiento basados en seguimiento telefónico no son efectivos para alcanzar la durabilidad de los efectos tras RR y recomienda la realización periódica de sesiones presenciales. Por su parte, estudios más recientes no han aportado demasiada luz sobre cuál es la estrategia mejor.

Otra revisión publicada en 2014, que incluye seis estudios sobre pacientes EPOC, concluye que las intervenciones de mantenimiento son muy heterogéneas en cuanto a su duración (iban de 8 a 64 semanas) y características (utilizaban control telefónico, sesiones presenciales o una combinación de ambos), pero que en todos los casos conseguían efectos modestos tras la finalización de los programas, que no se mantenían en el tiempo (126). Así mismo, en un metanálisis publicado en 2013, que evaluaba sólo estudios que hubieran analizado estrategias tras RR de al menos seis meses de duración, se reportó un mantenimiento de la capacidad de ejercicio sólo tras la finalización de los programas, que se perdía a losseis meses. No se hallaron diferencias ni a los 6 ni a los 12 meses, en otras variables como calidad de vida, el consumo de recursos sanitarios o el nivel de actividad física (AF) (127). Ambas publicaciones coinciden en que los resultados se deben por un lado, a la heterogeneidad de las intervenciones y por otro, a la no inclusión de actuaciones dirigidas a modificar los hábitos de vida de los pacientes.

\section{Estrategias de control durante las intervenciones de mantenimiento}

Se han publicado estudios que analizan estrategias diversas para el control de los pacientes en el mantenimiento tras la RR, tales como:

- Sesiones presenciales periódicas (128-132).

- Control telefónico (133-136).

- Apoyo en la comunidad (137).

- Telemedicina (138).

\section{Realización de sesiones presenciales periódicas}

En relación al empleo de sesiones presenciales periódicas en las que se incluye educación, pautas de automanejo y recomendación y orientación en la práctica de ejercicio físico, junto con la realización de entrenamiento, asemejando una sesión del programa de RR previo, 
su eficacia radica fundamentalmente en la duración del programa y la frecuencia de estas sesiones.

Programas de mantenimiento de corta duración o con pocas sesiones presenciales, no encontraron diferencias en la pérdida de los efectos tras la RR sobre la capacidad de ejercicio y la calidad de vida entre los sujetos sometidos al mantenimiento y los controles $(130,131)$ En este sentido, Wilson y col. (131) no hallaron diferencias al aplicar un mantenimiento de un año con una sesión presencial cada tres meses, así como tampoco lo hicieron Linneberg y col. (130), al aplicar seis sesiones durante un año (las tres primeras con una periodicidad quincenal y las siguientes, más distanciadas).

En contraposición, Ringbaek y col. (128) demostraron que un programa de mantenimiento prolongado (un año) en el que se realizaban sesiones presenciales semanales durante los primeros seis meses y quincenales en los seis meses siguientes, consiguió mantener los efectos sobre la capacidad de ejercicio en el grupo experimental, incluso hasta seis meses después de finalizar el programa. Sin embargo, esta estrategia intensiva no obtuvo diferencias entre los grupos en cuanto a la calidad de vida o las exacerbaciones. Por su parte, Effing y col. (132), aplicando una sesión presencial semanal durante cinco meses, también encontraron beneficios en el mantenimiento de la capacidad de ejercicio y el número de pasos semanales, así como en la disnea, aunque no hallaron diferencias en cuanto a calidad de vida.

El empleo de materiales de apoyo como folletos explicativos de cuánto y cómo deben realizarse los ejercicios de mantenimiento, acompañados de la cumplimentación de un diario que recoja los logros alcanzados, parece ser interesante a corto plazo (129). La aplicación de esta herramienta en un programa de mantenimiento de tres meses de duración en el que se incluía una sesión presencial semanal en el grupo experimental, consiguió mantener los resultados sobre la capacidad y ejercicio alcanzados tras el programa de RR. No se observaron diferencias entre grupo control y experimental en estos resultados, ya que el nivel de utilización del documento aportado y la cumplimentación del diario asociado, fue similar en todos los pacientes, lo que hace pensar que el añadir la sesión presencial no tuvo efecto adicional (129).

\section{Control telefónico}

El establecimiento de pautas para continuar con el entrenamiento de forma autónoma tras la finalización de los programas, asociado a control telefónico periódico para evaluar el nivel de adhesión, detectar y resolver problemas, así como motivar a los pacientes, es otra de las estrategias utilizadas, con resultados variables entre diferentes estudios.

Un programa basado en pautas de marcha semanal, con control telefónico cada cuatro semanas, consiguió mejorar la capacidad de ejercicio y la calidad de vida tras la finalización del programa, aunque no se evaluó su efecto a más largo plazo (133). Sin embargo, otro programa de mantenimiento también basado en la recomendación de caminar, pero sin pautas concretas, aunque con control telefónico semanal, no reportó efectos positivos sobre la capacidad de ejercicio, la calidad de vida o las exacerbaciones, si bien, sí mejoró el resultado en la disnea percibida o la calidad de vida en aquellos sujetos que indicaban caminar más tiempo diario (134). Por tanto, en este caso parece que fue más beneficioso el aporte de pautas precisas sobre la práctica de caminata diaria que el control telefónico en sí mismo. Otro estudio basado en la realización de marcha con control telefónico semanal durante tres meses obtuvo beneficios en el mantenimiento de la capacidad de ejercicio al finalizar el 
programa, pero no a los 12 meses, puesto que además, no se observaron cambios en el nivel de actividad física de los pacientes (135).

Por su parte, un programa de mayor duración (seis meses) con control telefónico mensual, pero en el que los sujetos debían realizar un plan de ejercicio, no marcha, no reportó beneficios ni a los $6 \mathrm{ni}$ a los 12 meses (136).

\section{Apoyo en la comunidad}

En un estudio piloto tras RR de cuatro semanas, realizaron un programa de mantenimiento basado en apoyo social con grupos colaborativos, que ofertaban sesiones de ejercicio semanal, educación y soporte psicológico durante 12 meses. No se establecían pautas concretas, pero los sujetos del grupo experimental tenían la posibilidad de acudir a este servicio cuando quisieran. Se obtuvo una duración de los efectos de la RR durante los 12 meses, tanto en la capacidad de ejercicio, como en la calidad de vida. Los sujetos que alcanzaron estos resultados fueron los que además, habían modificado su nivel de actividad física en relación a la situación previa a (137).

\section{Telemedicina}

El uso de herramientas de telemedicina está teniendo auge en el mantenimiento de los efectos tras RR (72).

En un estudio realizado en el Hospital Clinic (Barcelona) (138), se analizaron los efectos de un programa de mantenimiento mediante telemedicina, tras un programa ambulatorio de RR de ocho semanas, basado en una aplicación para dispositivos móviles, con feed-back personalizado y refuerzo mediante SMS tres veces por semana. Tras un seguimiento de casi dos años, los pacientes con adhesión a la herramienta consiguieron aumentar su nivel de actividad física y mantuvieron los efectos sobre la capacidad de ejercicio.

Tipo de actividad efectuada en los programas de mantenimiento

La actividad pautada en la fase de mantenimiento puede dirigirse hacia la realización de tablas de fuerza/resistencia en domicilio, replicando las sesiones de $\operatorname{RR}(128,130,131,136,137)$, ejercicio mediante caminar siguiendo pautas más o menos precisas $(133,134)$ o una combinación de ambos $(129,132,135,138)$.

En general, se observa un mayor mantenimiento de los efectos tras RR cuando se utilizan programas de marcha pautados (133) o en aquellos que consigue modificar el nivel de actividad física de los pacientes $(134,137,138)$, lo que concuerda con las conclusiones de las últimas revisiones sobre el tema $(126,127)$.

\subsection{El mantenimiento de los efectos tras la Rehabilitación Respiratoria autoadministrada}

En la revisión publicada por Vieira y col. en 2010 (98) concluyen que son necesarios más estudios para determinar el mantenimiento de los efectos tras la RR autoadministrada.

Posteriormente, varios estudios han reportado resultados positivos al comparar los sujetos sometidos al programa autoadministrado con un grupo control $(101,102,109)$. Resqueti y col. (101) encontraron una duración de los efectos sobre la capacidad de ejercicio y la calidad de vida en EPOC severos y muy severos, tras seis meses de la finalización de un programa domiciliario, con una intervención mínima de mantenimiento (llamada telefónica mensual). Por otro lado, el programa musical diseñado por Liu y col. (102), utilizado en un estudio posterior (109), 
consiguió una alta adherencia de los pacientes al programa (90\%) durante un seguimiento de 12 meses sin apenas feed-back (sólo una sesión de supervisión cada tres meses en consulta), lo que permitió un mantenimiento de los efectos sobre la capacidad de ejercicio, la calidad de vida (102) y la reducción en los niveles de inflamación sistémica (109).

Por otro lado, no se han encontrado diferencias en la duración de los efectos entre RR ambulatoria y domiciliaria, tanto al incorporar un programa de mantenimiento $(99,136)$ como al no incorporarlo $(100,107)$.

\section{Actividad física y EPOC. Un problema por resolver}

\subsection{Actividad física: algunos datos generales}

El American College of Sport Medicine (ACSM) define la actividad física como el "conjunto de movimientos corporales, realizados mediante la contracción de músculos esqueléticos, que origina un consumo energético" (139). A su vez, clasifica las actividades según su intensidad en cuatro categorías, basadas en el consumo energético necesario para realizarlas (Tabla 1): actividades de muy baja intensidad, baja intensidad, intensidad moderada y actividades vigorosas (139).

En el consenso del ACSM y la American Heart Association (AHA) publicado en 2007, sobre recomendaciones de actividad física para mantener una vida saludable en ancianos y enfermos crónicos, aconsejan la realización de 30 minutos de ejercicio moderado, cinco días a la semana, o bien, 20 minutos de ejercicio intenso, tres días a la semana (140). Esto es aplicable a los individuos sanos de más de 65 años y a enfermos crónicos de más de 50 años. De este modo, se consideran individuos sedentarios a aquellos que no alcanzan estos objetivos de actividad semanal (140).

Otro parámetro para las recomendaciones de actividad física diaria es en base al número de pasos caminados, puesto que el ejercicio más fácil, accesible y practicado es caminar (141). En este sentido, lo aconsejable es alcanzar entre 7.000 y 10.000 pasos diarios (88). Tudor-Locke y col. establecieron una clasificación de los individuos en base al número de pasos diarios que caminaban: sedentarios ( $<5.000$ pasos/día), individuos con baja actividad (5.000-7.499 pasos/ día), individuos con actividad moderada (7.500-9.999 pasos/día), activos (10.000-12.499 pasos/ día) y muy activos (>12.500 pasos/día) (142).

No obstante, a pesar de estas recomendaciones ampliamente extendidas, el nivel de AF de la población general no alcanza los valores mínimos en un alto porcentaje. Hallal y col. (143),

Tabla 1. Clasificación de las actividades según su intensidad.

\begin{tabular}{|c|c|c|}
\hline \multirow{2}{*}{ Tipo de actividad } & \multicolumn{2}{c|}{ Consumo energético } \\
\cline { 2 - 3 } & $\% \mathrm{VO}_{2} \max$ & METS \\
\hline Muy baja intensidad (sentado sin movimiento) & $<20 \%$ & $<1,5$ \\
\hline Baja intensidad (sentado con movimiento o de pie sin movimiento) & $20-40 \%$ & $1,5-3$ \\
\hline Intensidad moderada (de pie con movimiento ligero o desplazamiento) & $41-60 \%$ & $3,1-6$ \\
\hline Actividades vigorosas (actividades deportivas, tareas domésticas pesadas, \\
soporte de pesos) & $>60 \%$ & $>6$ \\
\hline
\end{tabular}

Clasificación establecida por el American College of Sport Medicine de las actividades, según el consumo energético requerido para realizarlas, medido como porcentaje del consumo máximo de oxígeno $\left(\% \mathrm{VO}_{2} \max \right)$ y por los equivalentes metabólicos (METS) (139). 
en un estudio poblacional que evaluaba el nivel de AF de la población de 122 países, hallaron que el porcentaje de individuos inactivos era del 31\% y que este valor ascendía al $43 \%$ en los países mediterráneos. Por su parte, Bohamon y col. (144) publicaron en 2007 un metanálisis en el que incluían 42 estudios que evaluaban el nivel de AF de la población mediante el número de pasos diarios. El análisis se llevó a cabo sobre una muestra total de 6.000 sujetos, encontrando que la media de pasos/día fue de 9.484, descendiendo hasta 6.565 en los mayores de 65 años, con lo que concluyen que especialmente en este grupo poblacional, no se alcanzan los niveles de pasos recomendados (144).

En España, los niveles de sedentarismo son aún elevados, aunque parece que se está mejorando. Según el Informe Anual del Ministerio de Sanidad y Política Social de 2015, el 44,4\% de los españoles mayores de 16 años se declaraban sedentarios, si bien, este dato era del $57,7 \%$ en 1993 (145).

Por otro lado, además del nivel de AF, hay que tener en cuenta los hábitos de vida cotidianos de los individuos, diferenciando éstos en comportamientos sedentarios y no sedentarios. Se considera que un individuo presenta un comportamiento sedentario si pasa el mayor tiempo del día efectuando actividades que supongan un coste energético inferior a 1,5 METS (tumbado o sentado sin movimiento) (146), independientemente de que realice la cantidad de ejercicio recomendada. Se ha reportado que entre el 46 y el $59 \%$ de la población general presenta un comportamiento sedentario (146).

Un parámetro objetivo que integra nivel de AF y comportamiento de los individuos es el denominado Physical Activity Level (PAL), que se obtiene como el cociente entre el consumo energético total en un día y el consumo energético durante el reposo nocturno (147). La OMS clasifica a los sujetos según su PAL en muy inactivos $(P A L<1,4)$, sedentarios $(1,4<P A L<1,7)$ y activos (PAL>1,7) (147).

\subsection{Nivel de actividad física en los pacientes EPOC}

En la mayoría de los pacientes, la EPOC comienza a manifestarse y por tanto, se diagnostica a partir de la cuarta década de la vida. En muchos de estos casos, desde este momento, el individuo entra en un "círculo vicioso" de disnea, sedentarismo y desacondicionamiento físico (97,148-150) (Figura 3). De este modo, el paciente EPOC tiende a evitar la realización de tareas que produzcan disnea, disminuyendo su nivel global de actividad. Esto conduce a una reducción progresiva en la fuerza y resistencia musculares y consecuentemente, a mayor disnea. Así, entran en este "círculo vicioso" que culmina en ocasiones, con aislamiento social y depresión (151).

El nivel de AF en la EPOC se ha evaluado mediante herramientas objetivas (acelerómetros y podómetros) y subjetivas (cuestionarios y diarios de actividad). Aunque en esencia, los resultados finales de baja actividad en los pacientes se han obtenido con ambos tipos de herramientas, existen diferencias en cuanto a la cuantificación. En una revisión sistemática publicada en 2011, que incluía 47 estudios que analizaban AF en pacientes EPOC mediante cualquier herramienta de evaluación, hallaron que el nivel de actividad era un $70 \%$ menor en los pacientes que en controles sanos si se medía mediante herramientas objetivas y un $57 \%$ si se empleaban herramientas subjetivas (152). Se considera que el empleo de cuestionarios y diarios de actividad tiende a sobrestimar los datos (153) y además, la información puede verse afectada por un sesgo cognitivo, influido por la memoria y la percepción de los pacientes (152). Sin embargo, el empleo combinado de dispositivos objetivos y cuestionarios o diarios de 
Figura 3. Círculo vicioso de disnea-sedentarismo-desacondicionamiento muscular en el paciente EPOC.

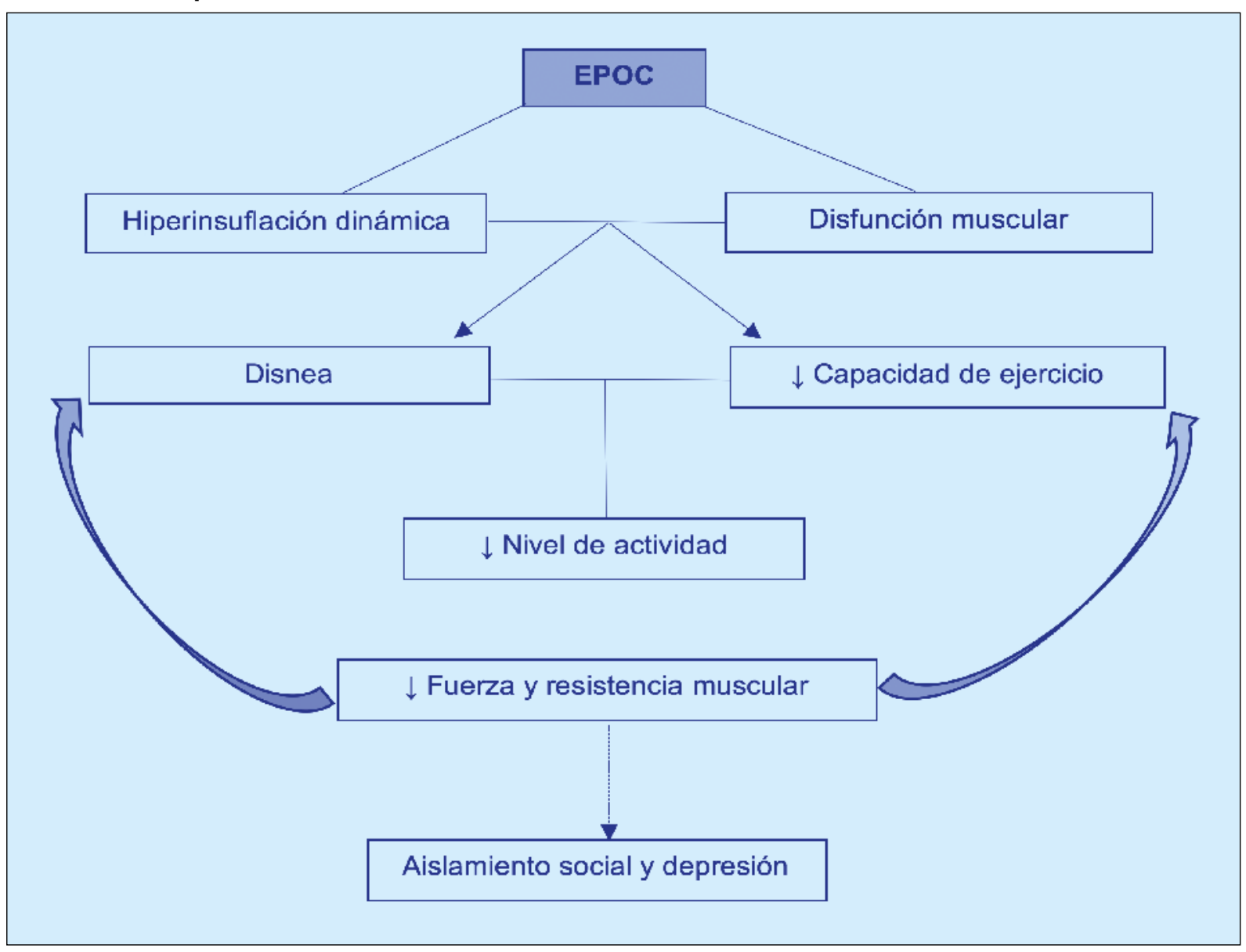

actividad aporta una información más completa $(153,154)$ y permite un mayor conocimiento individualizado de las características y las barreras de los pacientes al ejercicio (155).

Entre los estudios que emplearon herramientas objetivas para evaluar el nivel de AF en EPOC, hay un consenso en cuanto a que la duración y la intensidad de las actividades que realizan los pacientes son menores que en controles sanos de la misma edad (155-159), incluso en EPOC leves y moderados recién diagnosticados (160), así como en el gasto energético total $(157,159)$. Pitta y col. (159) encontraron que el nivel de AF en individuos EPOC era un $55 \%$ más bajo que en controles sanos, pasando más horas sentados y tumbados al día y efectuando actividades un $75 \%$ menos intensas. Por su parte, Eliason y col. (158) observaron también un menor tiempo e intensidad de las actividades de 75 sujetos con EPOC estable, si bien, las diferencias con los controles no fueron significativas con los EPOC leves. En este estudio, concluyeron que sólo el $27 \%$ de los EPOC leves realizaban la cantidad mínima de actividad recomendada semanal (150 minutos de ejercicio moderado), mientras que en el grupo de EPOC moderados y severos, este porcentaje se reducía aún más (por debajo del $20 \%$ ), frente al $40 \%$ que presentaban los controles. Por otro lado, en otro estudio se reportó que el $80 \%$ de los sujetos EPOC de la muestra caminaban al menos 30 minutos al día, pero que sólo el $29 \%$ lo realizaban a la intensidad adecuada, lo que indicaba que podían cumplir las recomendaciones de tiempo de ejercicio pero no de intensidad (161). No obstante, parece haber una asociación directa entre el tiempo que caminan al día estos pacientes y la velocidad de marcha (162).

También se ha observado un menor número de pasos/día de los pacientes EPOC en relación a controles sanos de la misma edad $(157,163,164)$ y a otros enfermos crónicos $(165)$, con una 
diferencia más acentuada en pacientes severos y muy severos (163). En el estudio multicéntrico de Troosters y col. (157), que incluía pacientes de Bélgica, Italia y Estados Unidos, encontraron que el número de pasos/día era de un $60 \%$ en relación a los controles sanos. Así mismo, en la mayoría de estos estudios $(163,164)$, la media de pasos/día de los sujetos no alcanzaba los 5.000 pasos, valor de corte que determina el nivel de sedentarismo (142).

Por su parte, utilizando herramientas no objetivas de evaluación de AF, en un estudio epidemiológico realizado en Suecia sobre más de 600 pacientes EPOC, se encontró que el nivel de AF era menor que en los individuos sanos, así como en relación a otras enfermedades crónicas como la artritis o la diabetes (166). En este estudio, hallaron que el $80 \%$ de los pacientes realizaban menos de dos horas semanales de ejercicio físico moderado.

Un dato interesante, reportado por Gouzi y col. (167) es que el declive en el nivel de AF en la EPOC comienza antes que en sanos y puede preceder el diagnóstico de la enfermedad e incluso a la aparición de disnea. En 2011 evaluaron la evolución del nivel de AF a lo largo de los años de 129 pacientes EPOC, mediante un sistema indirecto basado en entrevista y la cumplimentación de un cuestionario (QUANTAP System) y hallaron que el declive en el nivel de AF comenzaba de media a los 45 años en los pacientes, frente a los 55 años en sanos. Justifican este hallazgo indicando que probablemente el desarrollo de una disfunción muscular periférica asociada al tabaquismo sea la responsable del cese en la actividad de estos sujetos, incluso antes de comenzar con los primeros síntomas respiratorios (167).

\subsection{Factores que influyen en el bajo nivel de actividad física en los pacientes con EPOC}

\section{Función pulmonar y severidad de la enfermedad}

La asociación entre el nivel de AF y la unción pulmonar se ha analizado en diversos estudios. Muchos hallaron una correlación significativa y directa con el FEV $(152,161,163,164,168-173)$, mientras que en otros no se demostró asociación alguna (161,174-177). García-Río y col. (178) no hallaron asociación entre los cambios en el nivel de AF y el deterioro en la función pulmonar en una cohorte de 173 pacientes con EPOC españoles, a los que se les siguió entre cinco y ocho años. Troosters y col. (157) encontraron asociación entre el FEV y los pasos caminados, pero no con el consumo energético ni con el tiempo dedicado a actividades moderadas-intensas.

También se ha analizado la relación con otras variables que caracterizan la función pulmonar, hallando asociación significativa con la capacidad de difusión pulmonar $(160,179)$ y la máxima ventilación voluntaria (180). Así mismo, García-Río y col. (181) encontraron en una muestra de 89 pacientes con EPOC, que todos los individuos que presentaban hiperinsuflación dinámica tenían un bajo nivel de AF, manteniéndose una correlación significativa entre ambas variables.

Gimeno-Santos y col. (182), en una exhaustiva revisión sistemática publicada en 2014, analizaron más de 50 estudios y concluyeron que no hay datos consistentes para determinar la influencia del $\mathrm{FEV}_{1}$ en el nivel de AF de los pacientes EPOC y que la evidencia es más potente en cuanto a hiperinsuflación dinámica e intercambio de gases.

Por otro lado, atendiendo a la severidad de la EPOC según la clasificación clásica GOLD (43), se ha demostrado que el nivel de AF disminuye a partir del estadio II (157,183), observándose mayor inactividad en los pacientes más severos (151,157,168,183-185) (Gráfica 1). Watz y col. (183) encontraron que la mayor proporción de individuos muy inactivos (PAL $<1,4 \mathrm{METS}$ ) se observaba en los pacientes con GOLD III y IV. Por su parte, Jehm y col. (184), en una muestra 
Grafica 1. Nivel de actividad física de los pacientes con EPOC según la clasificación GOLD.

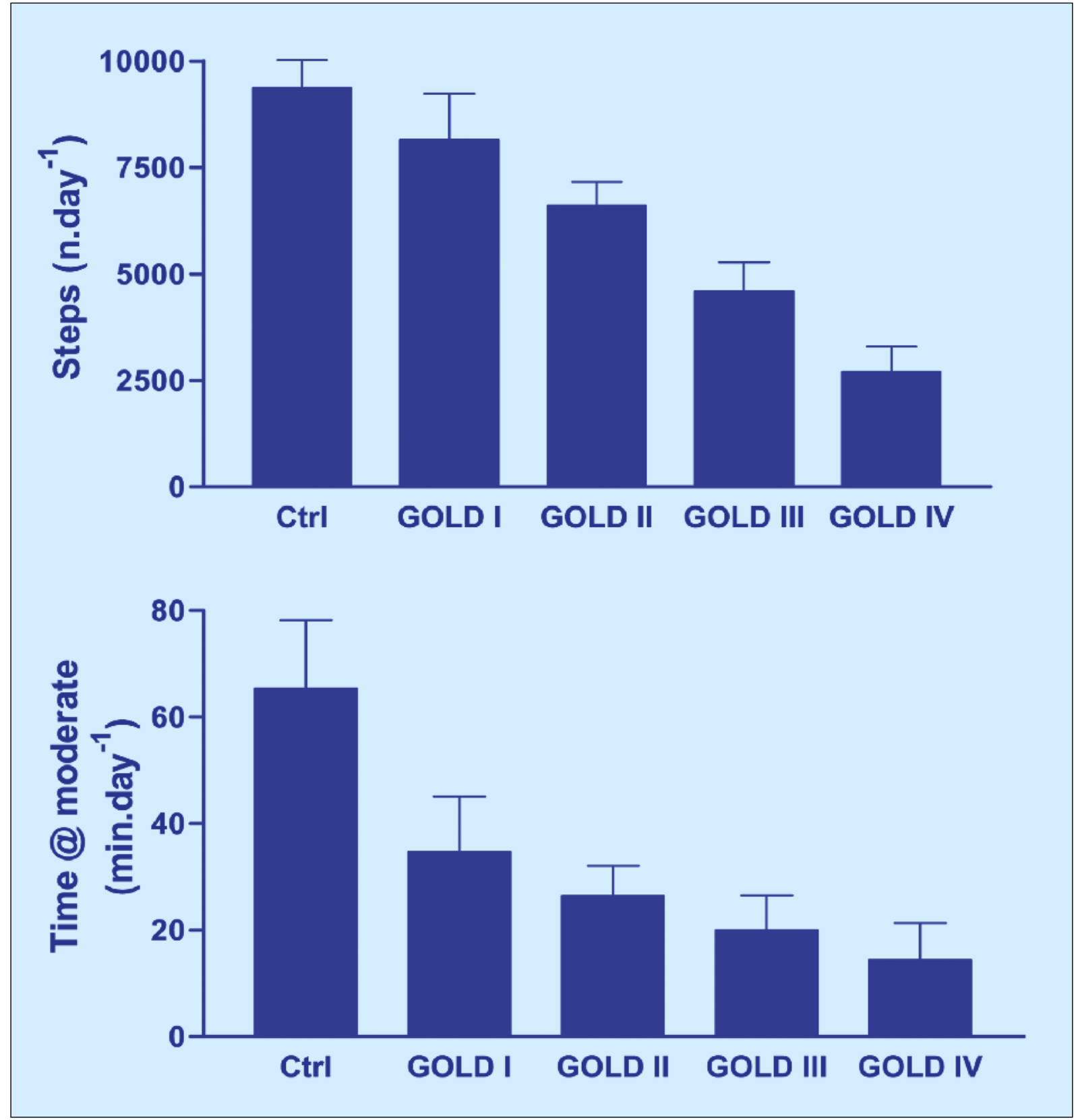

Gráfica extraída del estudio de Troosters y col., 2010 (157).

Representa los pasos/día (gráfica superior) y el tiempo diario dedicado a actividades de intensidad moderada (gráfica inferior) en sujetos EPOC según su severidad (clasificación de la Global Obstructive LUng Disease Iniciative), frente a sujetos controles sanos. Se observa que ambas variables son significativamente inferiores en los sujetos EPOC frente a los controles y a su vez, menor en individuos más severos.

(*) $p<0,05$

de 107 individuos con EPOC, hallaron que los sujetos con GOLD II caminaban significativamente más tiempo y a mayor velocidad que los pacientes con GOLD III y IV. Así mismo, según la clasificación GOLD de 2011 (46), Durheim y col. (186) observaron un menor nivel de AF en individuos en la categoría D.

Según el Índice BODE (44), también se ha demostrado asociación entre mayor severidad de la enfermedad y menor nivel de AF $(183,185,187)$. Mantoani y col. (187), no hallaron correlación significativa éntre la puntuación del Índice BODE y el nivel de AF, pero al dividir la muestra en pacientes leves-moderados (cuartiles 1 y 2 del Índice BODE) y severos-muy severos (cuartiles 3 
y 4), sí observaron que los sujetos más graves tenían menor gasto energético total y dedicaban menor tiempo a realizar actividades moderadas-intensas.

Sin embargo, a pesar de que la evidencia parece declinarse a una relación entre severidad de la enfermedad y nivel de AF en la EPOC, algunos estudios no han encontrado esta asociación ni con la estratificación según GOLD $(180)$ ni con el Índice BODE $(161,174)$.

\section{Capacidad de ejercicio}

Todos los estudios analizados hallaron una asociación significativa y directa entre el nivel de AF y la capacidad de ejercicio en los pacientes EPOC, especialmente evaluada mediante el 6MWT $(124,169,175-177,179,180,187-190,196,198,201,206-, 208)$. Hill y col. (190) encontraron una correlación significativa entre el nivel de AF evaluado mediante acelerometría y la distancia alcanzada tanto en un 6MWT como en una Prueba de Lanzadera Incremental (Incremental Shuttle Test, IST), más potente si se multiplicaba por el peso corporal, pero no con el $\mathrm{VO}_{2}$ pico. Además, observaron que los individuos que realizaban al menos 30 minutos de ejercicio moderado al día, alcanzaban una distancia en el IST superior a 450 metros, por lo que concluyen que el resultado de esta prueba podría ser un factor predictivo del nivel de AF de los individuos.

Por su parte, la revisión efectuada por Gimeno-Santos y col. (182) también concluye que la capacidad de ejercicio está relacionada con el nivel de AF en la EPOC. Por tanto, parece que la evidencia se inclina hacia una asociación entre nivel de AF y capacidad de ejercicio, pero no podemos determinar si la inactividad es causa o consecuencia de una menor tolerancia al esfuerzo.

\section{Disnea y exacerbaciones}

Varios estudios han reportado una asociación significativa e inversa entre la disnea basal y el nivel de AF en la EPOC $(162,163,168,171,184,189)$, mientras que en otros no observaron correlación alguna $(161,170,174,175,179)$. Polkey y col. (191) indicaron que la hipotrofia del cuádriceps asociada a la inactividad generaba una producción precoz de lactato durante el ejercicio, lo que podía ser un factor influyente en la aparición de disnea y contribuir así al "círculo vicioso" de disnea-sedentarismo observado en estos pacientes.

Por otro lado, parece que tras una exacerbación se observa una reducción del nivel de AF (192-194). En un estudio reciente, Alahmari y col. (193) encontraron que tras una exacerbación, la pérdida en pasos/día y tiempo fuera de casa era mayor en los individuos que habían tenido más de dos reagudizaciones en los 12 meses previos. Así mismo, Pitta y col. (192) observaron que tras un mes de sufrir un ingreso hospitalario por exacerbación, a pesar de que el tiempo y la intensidad de las actividades que realizaban los pacientes iban aumentando, aún no se alcanzaban los valores previos. Sin embargo, García-Aymerich y col. (176) no hallaron asociación entre el nivel de AF en pacientes EPOC severos y muy severos y las exacerbaciones previas.

En la revisión de Gimeno-Santos y col. (182) concluyen que las evidencias se decantan hacia la asociación entre disnea basal y exacerbaciones previas con el nivel de AF en la EPOC.

Edad e índice de masa corporal

Diversos estudios han reportado una asociación significativa e inversa entre el nivel de AF y la edad (157,172-176). Moy y col. (168) observaron que el nivel de AF en los pacientes EPOC se reducía con el tiempo, así como el $\mathrm{FEV}_{1}$, pero el ritmo del deterioro en la actividad fue mayor que en la función pulmonar. 
En cuanto al IMC, no hay muchos estudios que hayan analizado su asociación con el nivel de AF en pacientes EPOC y los resultados no son concluyentes. Algunos determinaron una asociación significativa y directa $(161,195)$, mientras que en otros no hallaron relación alguna $(170,175,176)$.

En la revisión de Gimeno-Santos y col. (182) concluyen que no hay evidencias suficientes para determinar la asociación entre edad e IMC y nivel de AF en la EPOC.

Inflamación sistémica y comorbilidades

La presencia de comorbilidades puede ser un factor influyente en el bajo nivel de AF de los pacientes EPOC, especialmente la diabetes (176), las enfermedades cardiacas (185) y el síndrome metabólico (196). Esteban y col. (197) encontraron, en una cohorte de más de 500 pacientes EPOC, que los individuos con menor nivel de $A F$, evaluado mediante el tiempo que dedicaban a caminar a la semana, eran los que presentaban una mayor puntuación en el Índice de Charlson. Así mismo, Sievi y col. (198), en un estudio reciente, analizaron 250 pacientes EPOC y hallaron que los que tenían alguna comorbilidad asociada presentaban significativamente menor PAL, pasos/día y tiempo dedicado a actividades moderadas-intensas. Concluyen que la presencia de comorbilidades en la EPOC es un factor predictor de deterioro en el nivel de $A F$, independientemente de su número o tipología.

Así mismo, la inflamación sistémica se asocia significativamente y de forma inversa al nivel de AF en la EPOC $(152,179,182)$, independientemente de la severidad de la enfermedad (185). Ahora bien, no se sabe si el incremento en los marcadores inflamatorios es causa o consecuencia de la inactividad (152).

\section{Otros factores}

La necesidad de oxigenoterapia continua domiciliaria (OCD) es un factor que puede tener influencia en la actividad de los pacientes (171), especialmente fuera de casa (176).

Los factores psicológicos no han sido muy evaluados. Sin embargo, pueden influir en el nivel de AF de los pacientes EPOC, especialmente en relación a criterios de autoeficacia, automanejo, depresión-ansiedad y apoyo social (171). Altenburg y col. (169) hallaron que estos factores influían más en el nivel de AF de los pacientes leves-moderados que la función pulmonar o la capacidad de ejercicio, mientras que en los más severos se producía a la inversa. Últimamente, se han validado herramientas para conocer las dificultades y las adaptaciones de los pacientes ante la actividad física, al considerarse aspectos a tener en cuenta en la evaluación del nivel de AF en la EPOC (199).

Por otro lado, según una revisión publicada en 2012 que analiza las barreras autorreportadas por los pacientes para la práctica de ejercicio, se concluye que los factores principales que se identifican como limitantes son: la ansiedad y el miedo a la práctica deportiva; la aparición de disnea, fatiga o malestar durante y tras el ejercicio; la falta de tiempo; la presencia de otras enfermedades asociadas; las malas condiciones climatológicas; la falta de apoyo social y la desinformación acerca de los beneficios del ejercicio (97).

\subsection{Efectos de la inactividad en la EPOC}

Actualmente, los efectos nocivos de la inactividad en la EPOC están completamente demostrados (154). De este modo, las principales guías de práctica clínica de manejo de estos pacientes y los documentos de consenso, recomiendan realizar estrategias para incrementar su nivel de $\operatorname{AF}(72,81,83,155,200)$, incluso de forma precoz tras una exacerbación (34). La 
Iniciativa GOLD también incorpora esta recomendación desde 2007 (151) y la mantiene en sus posteriores versiones hasta la última actualización de 2016 (1).

Incremento en el riesgo de exacerbaciones y muerte

El valor pronóstico del nivel de actividad física en la EPOC se ha demostrado en diversos estudios $(170,177,178,186,192,197,201-203)$. En la revisión publicada por Gimeno-Santos y col. en 2014, concluyeron que son consistentes las evidencias de que la AF reduce las exacerbaciones y la mortalidad en la EPOC (182). Así mismo, en la Guía GesEPOC de 2012, se introduce el nivel de actividad física diario como un criterio más a tener en cuenta para establecer la gravedad de la enfermedad, según el número de minutos en los que llevan a cabo actividades moderadas (2).

García-Rio y col. (178) demostraron en una cohorte de 188 pacientes con EPOC estable que el nivel de AF, evaluado mediante acelerometría, fue el único predictor independiente de exacerbaciones y éste junto con las comorbilidades, de mortalidad. Así mismo, García-Aymerich y col. (203) hallaron, en una cohorte de más de 2.000 sujetos con EPOC, que el nivel de AF correlacionaba con las hospitalizaciones y la mortalidad, independientemente de factores como la edad, el IMC, la presencia de comorbilidades y la severidad de la enfermedad (Gráfica 2). Estos resultados son coherentes con los de Benzo y col. (202), quienes tras analizar a 177 enfermos de EPOC severa durante un año, concluyeron que el riesgo de exacerbaciones fue significativamente mayor en aquellos que realizaban menos de dos horas de ejercicio semanal. En un estudio reciente, también encontraron esta asociación entre el nivel de AF y las exacerbaciones y la mortalidad en los distintos estadios GOLD, corregido por edad y exacerbaciones previas (186).

Por su parte, Waschki y col. (177) demostraron, en una cohorte de 170 pacientes con EPOC leve-moderada a los que se les siguió durante cuatro años, que un menor nivel de actividad física diario conducía a un mayor porcentaje de mortalidad. Clasificaron a los sujetos según su PAL y hallaron que tras un periodo de seguimiento de cuatro años, habían fallecido el 31\% de los individuos muy inactivos, el $9 \%$ de los sedentarios y no se produjeron muertes entre los clasificados como activos. Además, este estudio muestra cómo la actividad física en pacientes EPOC podría ser un factor pronóstico de mortalidad más potente que el Índice BODE, el Índice ADO (Age, Dysnea and Obstruction) o el FEV 1 (177).

En esta misma línea, Moy y col. (188), basándose en que el nivel de AF y la inflamación sistémica son factores muy relevantes de pronóstico en la EPOC, diseñaron un índice combinado de nivel de AF y niveles inflamatorios (pasos/ proteína C reactiva y pasos/ interleucina), hallando una asociación significativa entre ambos con exacerbaciones y mortalidad, independientemente del $\mathrm{FEV}_{1}$. Concluyen que estos índices pueden ser predictores tan importantes como el Índice BODE.

Una de las actividades más evaluadas y recomendadas es caminar. García-Aymerich y col. (204) observaron que caminar cinco días por semana durante una hora reducía las exacerbaciones en individuos EPOC. Durheim y col. (186) afirmaron que los pacientes que caminaban al menos 4.000 pasos/día reducían el riesgo de mortalidad en los años siguientes, mientras que aquellos que caminaban menos de 200 pasos/día incrementaban significativamente el riesgo de muerte (Gráfica 3).

Por último, indicar que la modificación en los niveles de actividad también es un factor pronóstico de evolución $(186,197)$ y mortalidad en la EPOC (205). Esteban y col. (197) encontraron 
Gráfica 2. Asociación entre el nivel de actividad física en la EPOC y las hospitalizaciones/mortalidad.

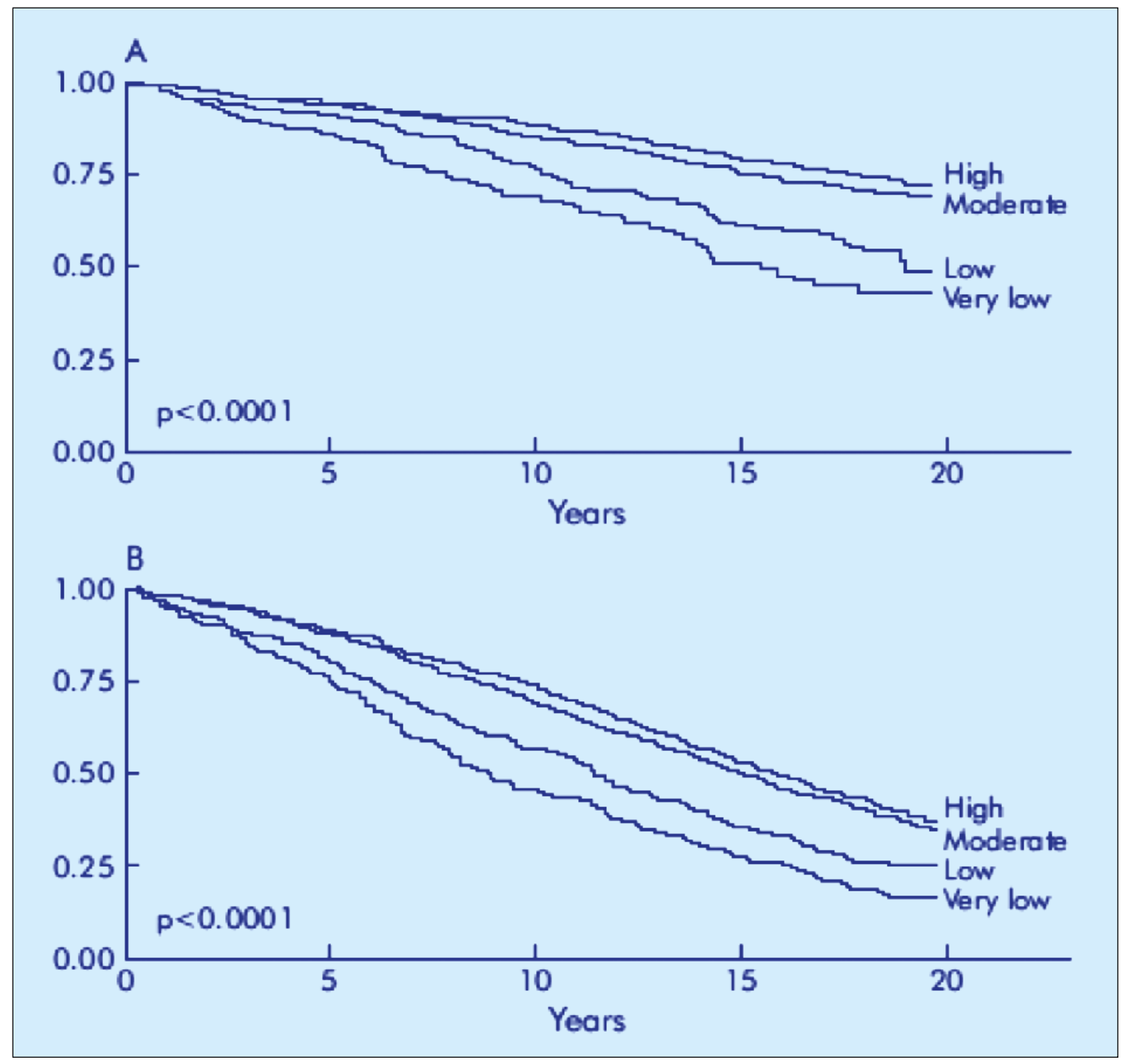

Gráfica extraída del estudio de García-Aymerich y caol, 2006 (203).

Asociación entre hospitalizaciones (A) y mortalidad (B) con el nivel de AF en la EPOC durante 20 años de seguimiento. Se observa que los individuos con alto nivel de actividad (High) son los que tienen menos hospitalizaciones y mortalidad, y que los individuos muy inactivos (Very low) son los que presentan peores resultados.

Very low: nada de actividad; Low: AF<2 horas semanales; Moderate: AF entre 2-4 horas semanales; High: AF>4 horas semanales.

que los individuos que habían incrementado su nivel de AF durante dos años, tenían menor riesgo de padecer exacerbaciones en los tres años siguientes, independientemente de la edad, el FEV 1 y las exacerbaciones previas.

Reducción en la calidad de vida

Diversos estudios encontraron una asociación significativa entre calidad de vida y pasos/día $(164,168,169)$ o el tiempo dedicado a caminar deprisa al día (206). Esteban y col. (197) han reportado que los individuos que caminaban más de $6 \mathrm{Km}$, al menos tres días por semana, tenían mejores puntuaciones en el St. George's Respiratory Questionaire (SGRQ). Este mismo grupo, en un estudio anterior, encontró que los pacientes que caminaban menos de dos 
horas al día presentaban una menor calidad de vida y en aquellos que incrementó el tiempo dedicado a caminar a la semana, alcanzaron significativamente mejores puntuaciones en el cuestionario, independientemente del $\mathrm{FEV}_{1}$ y las comorbilidades (207).

\subsection{Estrategias para aumentar el nivel de actividad física en los pacientes EPOC}

\section{La Rehabilitación Respiratoria como estrategia para modificar el nivel de actividad física}

A pesar de la mejora en la capacidad de ejercicio tras la RR, los programas clásicos no modifican el nivel de AF de los pacientes $\operatorname{EPOC}(155,208)$ o lo modifican mínimamente $(150,209)$, incluso aunque se incorporen recomendaciones específicas (210). Se ha reportado que la RR puede reducir el miedo a la práctica de ejercicio, pero esto no parece ser suficiente para influir en sus hábitos (211).

Las últimas publicaciones afirman que existe cierta controversia en este tema $(212,213)$ y que los estudios publicados presentan muy baja calidad metodológica, por lo que la evidencia no es suficiente para determinar el impacto de la RR en el nivel de AF en la EPOC $(182,212,213)$. En la revisión de Mantoani y col, sobre intervenciones para modificar el nivel de AF en la EPOC (212), hallaron que los estudios que mostraban un aumento en el nivel de AF de los pacientes utilizaban herramientas subjetivas de evaluación, concluyendo que la RR podía haber provocado un cambio en la percepción de actividad de los pacientes.

En ausencia de estrategias específicas, no se han encontrado diferencias entre programas ambulatorios y domiciliarios sobre el impacto en el nivel de AF de los pacientes (214), mientras

Gráfica 3. Riesgo de muerte en pacientes EPOC en función del número de pasos/día.

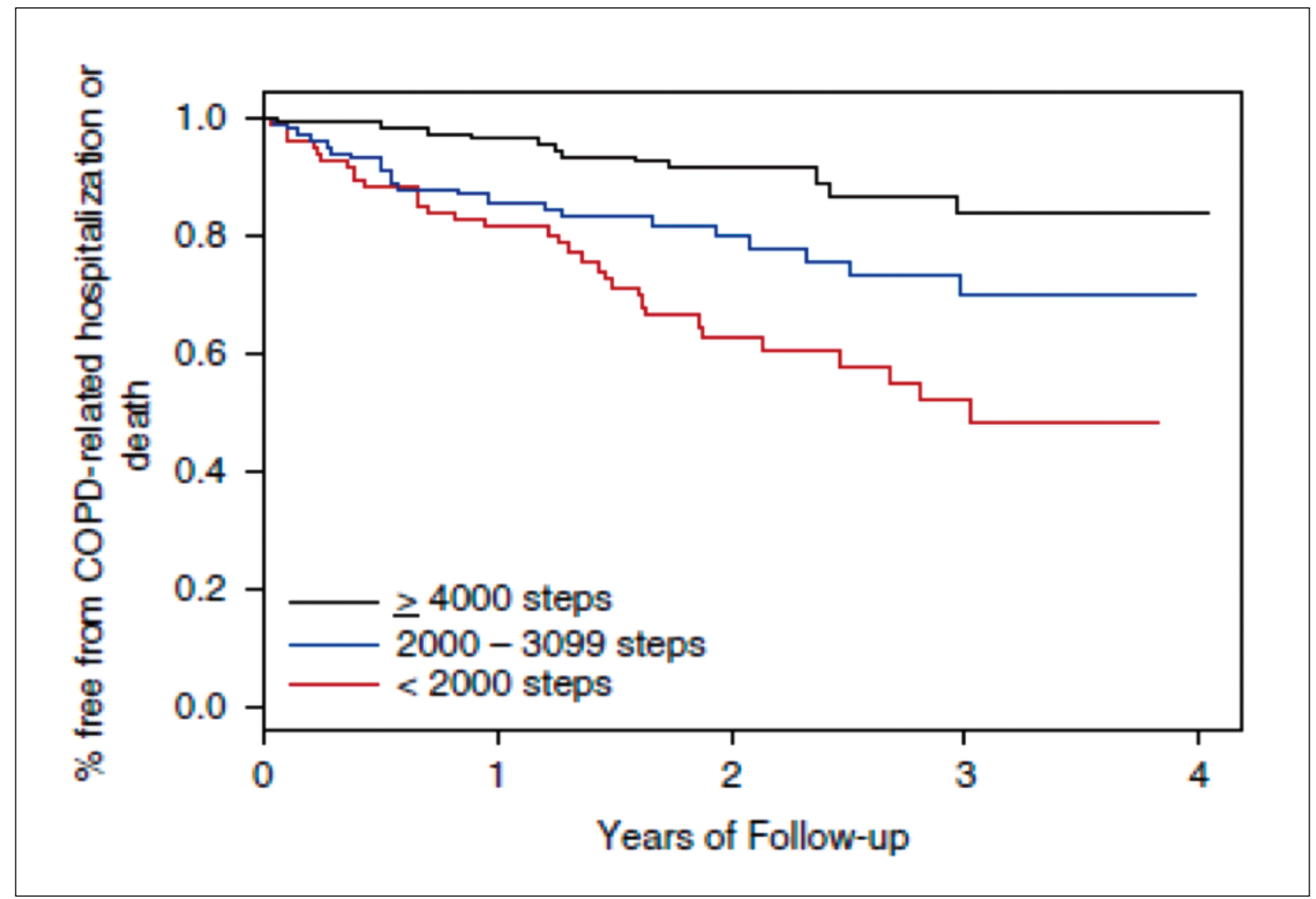

Grafica extraída del estudio de Durheim y col., 2015 (186).

Se observa que los pacientes que caminaban al menos 4.000 pasos/día tenían un menor riesgo de mortalidad en los cuatro años de seguimiento. Así mismo, aquellos que caminaban menos de 200 pasos/día presentaron un riesgo elevado. 
que su duración puede ser un factor influyente $(209,212,215)$. Pitta y col $(215)$, tras un programa ambulatorio de tres meses, que se prolongó otros tres meses para los individuos del grupo experimental, hallaron que los cambios en el tiempo y la velocidad de caminata diaria no se conseguían al finalizar la primera fase, pero sí en el grupo experimental tras el segundo período. Por tanto, concluyen que somos capaces de entrenar el músculo con intervenciones relativamente cortas, pero son necesarios programas más largos para conseguir modificar el comportamiento de los pacientes.

La realización de programas de RR de larga duración no es viable en la mayoría de los casos, por lo que es necesario buscar alternativas para incrementar el nivel de AF en los pacientes EPOC. Las principales guías de práctica clínica recomiendan la incorporación de estrategias específicas tras la RR $(72,81,83,155)$ aunque no hay consenso acerca de qué intervención es mejor (155). Por tanto, es necesaria una mayor investigación sobre el tema $(182,212,213)$, considerándose una línea de desarrollo prioritaria por la ATS y la ERS (125).

\section{Estrategias específicas asociadas a la Rehabilitación Respiratoria}

Se han publicado diversos estudios que evaluaban intervenciones tras un programa de RR para aumentar el nivel de AF en pacientes EPOC. Entre ellas, la incorporación de llamadas telefónicas periódicas $(135,214,216,217)$, el empleo de herramientas de telemedicina (138) y el apoyo en la comunidad (216).

Parece que las llamadas telefónicas periódicas para incentivar y motivar, como estrategia aislada, no consiguen modificar los hábitos de los pacientes $(78,135,214,217)$. Soicher y col. (214) reportaron que tras un programa de 12 semanas al que se incluyeron recomendaciones de continuar realizando el entrenamiento en domicilio y se efectuaban llamadas telefónicas motivacionales cada dos meses, el nivel de AF no se modificó, observando que sólo el $30 \%$ de los individuos realizaban más de dos horas de ejercicio moderado semanal. Por su parte, aumentar la frecuencia de las llamadas telefónicas a cada dos semanas tampoco ofreció resultados positivos en el estudio de Donesky-Cuenco y col. (217). En éste se observó que durante 12 meses, a partir del quinto mes se producía una reducción en el cumplimiento de la recomendación de caminar al menos 20 minutos, cuatro días a la semana.

Por otro lado, Zwerink y col. (218) incluyeron un programa de seis meses con una sesión de entrenamiento presencial semanal. Hallaron que el número de pasos/día había aumentado tras el programa completo y que perduraba en los 12 meses siguientes.

Así mismo, en el estudio de Barberán-García y col., referenciado en el apartado 3.2., consiguieron que los individuos que tenían adherencia a la aplicación móvil realizaran mayor número de horas de actividad al día durante un período de seguimiento de casi dos años tras un programa ambulatoRio de ocho semanas (138).

Por último, mencionar el estudio de Pleguezuelos y col. (216), que diseñaron un programa de circuitos urbanos en colaboración con el Ayuntamiento de Barcelona y evaluaron su efecto en modificar los niveles de actividad en pacientes EPOC severos y muy severos que habían participado en un programa ambulatorio de RR de 12 semanas. Tras 12 meses de seguimiento, se observó que aumentó significativamente el tiempo dedicado a caminar a la semana e incluso, este resultado se asoció con un mayor mantenimiento de la distancia alcanzada en un 6MWT.

\section{Estrategias independientes de la Rehabilitación Respiratoria}

En una revisión publicada en 2014 sobre estrategias independientes a la RR, dirigidas a aumentar el nivel de AF en pacientes EPOC, se concluye que parece que hay una cierta evidencia 
sobre la efectividad de las intervenciones, a pesar de su gran variabilidad. Se han realizado actuaciones que iban desde sesiones presenciales de educación y práctica de ejercicio, Ilamadas telefónicas, uso de diarios de actividad y herramientas de telemedicina. También se han empleado estrategias de cambio comportamental basadas en el aporte de información, el planteamiento de logros, los sistemas de feed-back como los monitores de actividad e intervenciones psicológicas específicas. La prescripción más habitual es la de practicar ejercicio, al menos tres días en semana durante 20 minutos, fundamentalmente caminando. Los autores afirman que son más útiles las intervenciones basadas en caminar y las que utilizan actuaciones de cambio comportamental (219).

En la población general, las estrategias dirigidas a incorporar cambios del comportamiento son eficaces para aumentar el nivel de AF de los individuos (220). Así mismo, en los pacientes EPOC el nivel de AF también está muy condicionado por el comportamiento y no sólo por su capacidad o posibilidades, es decir, en muchos casos no se muestran más activos porque no quieren y no porque no puedan (150). Por tanto, las intervenciones tienen que ir orientadas a incorporar un cambio de hábitos (150). En esta misma línea, Hill y col. (154), en una reciente revisión, afirman que las actuaciones dirigidas a aumentar el nivel de AF en la EPOC deben tener un doble enfoque, práctica de ejercicio semanal y modificación de las rutinas diarias. Estas conclusiones se ven reforzadas por Mantoani y col. (212), en la revisión mencionada anteriormente.

En relación a intervenciones para modificar el comportamiento, en otra revisión publicada en 2014 sobre patologías crónicas, concluyen que las más eficaces son las educacionales, las basadas en la consecución de logros, los sistemas de retroalimentación con seguimiento del personal sanitario y el soporte social (221).

En cuanto al empleo de monitores de actividad como sistemas de feed-back en los pacientes EPOC, hasta 2012 no había suficiente evidencia de su eficacia (222). No obstante, en la revisión de Wilson y col. (219) afirman que los podómetros son dispositivos útiles y más eficaces que los acelerómetros, como sistemas de retroalimentación en los programas de incremento del nivel de AF en individuos con EPOC. Por su parte, Mantoani y col. (212) también afirman que los podómetros se han mostrado eficaces como elementos de incentivo y adhesión a la actividad.

\section{Los podómetros. Una herramienta útil en el entrenamiento y la modificación del nivel de actividad}

\subsection{El empleo de podómetros en diversos grupos poblacionales}

Los podómetros son dispositivos simples y baratos que miden los pasos mediante la detección de movimiento en un plano vertical, utilizando un mecanismo de resorte (Figura 4). Son de fácil manejo y transporte, fiables en el conteo de pasos, y pueden proporcionar datos sobre la velocidad de caminata y el consumo calórico total, si bien, su fiabilidad en el reporte de esta información es menor (155). Además, su sensibilidad se reduce cuando la velocidad de caminata es baja $(155,223)$. Los podómetros pueden infraestimar el conteo de pasos cuando se camina a velocidades inferiores a $3,79 \mathrm{Km} / \mathrm{h}$, por lo que hay que utilizarlos con cautela como sistemas de registro de actividad en individuos que caminan lento (223). No obstante, Ewald y col. (224) demostraron su validez para evaluar el nivel de AF en ancianos sanos.

Por otro lado, los podómetros son herramientas muy útiles como sistemas de feed-back en programas de incremento de actividad en distintos grupos poblacionales $(222,225)$. Bravata y col. (225), en un metanálisis, encontraron que el uso de podómetros en adultos sanos 
Figura 4. Distintos modelos de podómetros digitales.

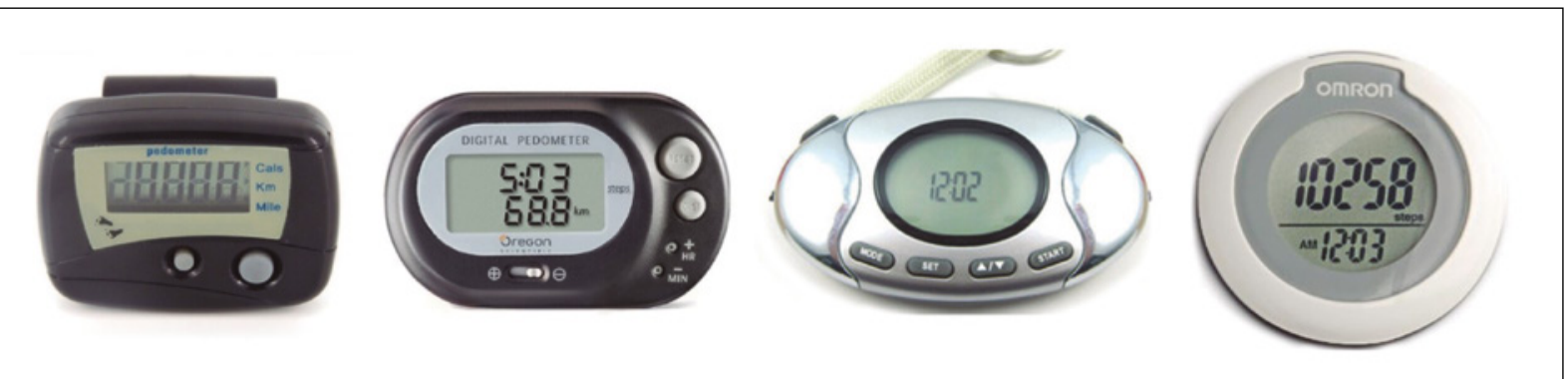

conducía a un incremento en el número de pasos por día. Por su parte, en la revisión de Vaes y col. (222) determinaron que el uso de podómetros es eficaz para aumentar AF y reducir el IMC en diabéticos.

Puesto que caminar es la actividad más sencilla y accesible para que los individuos realicen ejercicio y modifiquen sus hábitos (146), muchos programas de incremento de actividad se basan en aumentar el número de pasos por día. Por ello, la utilización de los podómetros ha sido descrita como sistema de registro y autocontrol en programas de incremento de actividad física en diversos grupos poblacionales (226-233). Su eficacia se ha demostrado en la población general (230), en ancianos sanos (227), en diabéticos (226), en cardiópatas (229), en pacientes con cáncer (228) y en individuos con arteriopatía periférica $(232,233)$, etc.

Algunos estudios asocian el empleo del podómetro a estrategias de cambio comportamental según distintas teorías, como la "Teoría de establecimiento de metas u objetivos" descrita por Locke (234) (Figura 5). Aplicando esta teoría y utilizando podómetros como sistema de retroalimentación y seguimiento telefónico semanal durante un programa de 12 semanas, Chan y col. (230) reportaron un incremento en el número de pasos/día en adultos sanos tras el programa y a los 12 meses.

\subsection{El empleo de podómetros en la EPOC}

Como herramienta para evaluar el nivel de actividad física

Como se ha expuesto en el apartado anterior, los podómetros pueden infraestimar el conteo de pasos cuando se camina a velocidad lenta. Sin embargo, se han utilizado en numerosos estudios en pacientes con EPOC para evaluar el nivel de AF habitual y tras una intervención $(132,148,164,169,170,172,174,188,235-242)$, así como durante y tras una exacerbación (193).

Troosters y col. (150), en una revisión de 2013, concluyen que los podómetros son una herramienta fiable en estos pacientes. Así mismo, en otra revisión anterior, Kocks y col. (243) afirman que los podómetros son dispositivos fáciles de utilizar, válidos, reproducibles y sensibles para evaluar AF en pacientes con EPOC.

En la literatura se pueden encontrar diversos estudios que demuestran la validez de los podómetros para cuantificar el número de pasos $(244,245)$, especialmente en caminata continua (246). Depew y col. (244) hallaron una correlación entre el número medio de pasos mediante un podómetro y el PAL en pacientes EPOC. Así mismo, encontraron una asociación entre un $\mathrm{PAL}<1,4$ y 4.580 pasos/día, por lo que concluyen que los individuos que caminan menos de 4.580 pasos diarios pueden considerarse inactivos. Por otro lado, Furlanetto y col. (245), en un estudio de validación de un podómetro en individuos con EPOC, reportaron una mayor fiabilidad en el conteo de pasos que con un acelerómetro cuando los individuos caminaban al 30\%, 60\% y $100 \%$ de la velocidad media alcanzada en 
un 6MWT, si bien, el registro podométrico sólo fue completamente fiable a la velocidad más alta.

Como sistemas de monitorización en programas de entrenamiento y de incremento del nivel de actividad física

Los podómetros se han utilizado como sistemas de monitorización y feed-back en programas de $R R$ autoadministrados $(107,108,247)$ y de mantenimiento tras RR $(133,135)$. Así mismo, como ya se ha indicado antes, los podómetros son útiles para aumentar el nivel de AF en pacientes con EPOC (212), más aún que los acelerómetros (219). De hecho, algunas guías de práctica clínica y consensos de expertos recomiendan el uso de podómetros y diarios de actividad para modificar los hábitos de vida en este tipo de pacientes $(78,155)$, así como herramienta complementaria en la monitorización y el seguimiento (200).

En varios estudios se han utilizado los podómetros como sistema de feed-back y registro asociados a las nuevas tecnologías (237-239). Moy y col. (239) desarrollaron un programa que almacenaba el registro de pasos semanales y reportaba retroalimentación individualizada. Este sistema fue modificándose y aplicando en estudios posteriores $(237,238)$.

Por otro lado, al igual que en otros grupos poblacionales, los podómetros se han utilizado en estrategias específicas de cambio comportamental para pacientes EPOC, como la "Teoría de establecimiento de metas u objetivos" de Locke (234) (Figura 5). En este tipo de intervenciones, los podómetros son empleados para aportar retroalimentación constante de la consecución de los logros del paciente, una de las fases clave de la intervención. Con esta finalidad se han utilizado en programas aislados de incremento de actividad $(148,236)$ y asociados a programas de $\operatorname{RR}(241,248)$. Se ha demostrado que programas de incremento de AF basados en intervenciones de cambio comportamental con podómetro, de 12 semanas de duración, consiguen mejorar la capacidad de ejercicio, la calidad de vida y el número de pasos semanales $(148,236)$.

Figura 5. Principios de la Teoría de establecimiento de metas u objetivos.

\section{Primera fase: La claridad}

Establecer una meta clara y concreta, centrada en el objetivo que se pretende conseguir.

\section{Segunda fase: Establecer retos}

Especialmente en el caso de metas a largo plazo, el hecho de fijar retos parciales aumenta la motivación.

\section{Tercera fase: El compromiso}

Para alcanzar la meta es necesario el compromiso de los implicados. Para conseguir éste, es preciso dialogar y consensuar acuerdos.

\section{Cuarta fase: La retroalimentación}

Aportar información acerca del grado de consecución de los logros para poder orientar los esfuerzos en la dirección adecuada.

\section{Quinta fase: La complejidad de las tareas}

En el caso de metas de complicado alcance será preciso establecer metas parciales, división y secuenciación de las actividades. 
Por su parte, asociar un programa de incremento de actividad basado en cambio comportamental con podómetro a un programa ambulatorio de RR de 12 semanas, consigue aumentar el nivel de actividad tras el programa, pero no añade efectos adicionales sobre la capacidad de ejercicio o la calidad de vida $(148,248)$.

Por último, indicar que a pesar de que los podómetros han demostrado ser herramientas útiles y se recomienda su uso, no hay directrices generales de cómo establecer la progresión en cuanto al incremento de pasos. Algunos estudios fijan un incremento concreto, de 400 pasos/día de media semanal (238), de 600 pasos/día (237) u 800 pasos/día (239). Otros estudios no establecen una progresión fija, sino en función de cada paciente $(148,236)$. Además, la evidencia sobre la duración del incremento en el nivel de actividad conseguido con estos programas es aún escasa y sería necesaria una mayor investigación (212).

\section{Consideraciones finales}

La EPOC es una enfermedad crónica que cursa con disnea e intolerancia al ejercicio, aspectos que se benefician en gran medida, de la RR. Sin embargo, la duración de sus efectos es limitada y a pesar de que la evidencia al respecto es amplia, la influencia de distintos tipos de entrenamiento, así como la concurrencia de otros factores en la duración de los efectos, no está clara. No obstante, lo que sí parece evidente, es que es necesario incorporar estrategias de mantenimiento tras la RR, aunque tampoco hay consenso acerca de qué intervenciones son más eficaces.

Por otro lado, el acceso de los pacientes a la RR es insuficiente y desigual. Además, los mayores beneficios los reportan programas de larga duración, inviables en la mayoría de los casos. Por tanto, se hace necesario buscar otras alternativas que reduzcan los costes y acerquen la RR a la comunidad. En este sentido, los programas autoadministrados cobran relevancia. Sin embargo, también se requieren más investigaciones para establecer directrices de actuación.

Otro aspecto a tener en cuenta es el nivel de AF de los pacientes EPOC, que es bajo y no se ve modificado tras la RR. Puesto que la inactividad influye negativamente en la evolución y el estado funcional de estos pacientes, es preciso incorporar estrategias específicas que modifiquen sus hábitos cotidianos, si bien, se requieren más estudios sobre intervenciones eficaces.

Los podómetros pueden ser herramientas útiles en la monitorización de los pacientes durante programas de incremento de actividad. Sin embargo, aún la evidencia disponible es insuficiente y en especial, hacen falta más estudios que permitan establecer pautas precisas de actuación en cuanto al uso de estos dispositivos. Además, son aún más escasos los estudios que utilizan el podómetro para la monitorización de programas de entrenamiento autoadministrado, con la consiguiente ausencia de consensos en cuanto a la metodología a seguir, tanto durante el desarrollo del programa como en la fase de mantenimiento. 


\section{OBJETIVOS}

En base al planteamiento anterior, se establecen dos objetivos principales para el desarrollo de esta tesis doctoral.

\section{Objetivo 1}

Describir los efectos de varios programas de Rehabilitación Respiratoria aplicados a pacientes EPOC y evaluar su evolución tras un período de seguimiento sin estrategias de mantenimiento. Analizar los factores influyentes en la pérdida de los efectos durante el seguimiento.

Este objetivo responde a la necesidad de aportar evidencia acerca de los factores que influyen en el mantenimiento de los beneficios obtenidos tras programas ambulatorios de RR, especialmente en relación a determinar estrategias de entrenamiento con beneficios más duraderos.

Para la consecución de este objetivo se ha llevado a cabo un estudio observacional, cuya metodología y resultados se describen en la Parte II de esta tesis. Aquí se indican sus objetivos.

\section{Estudio 1}

Efectos a corto y largo plazo de tres modalidades de programas clínicos de Rehabilitación Respiratoria en pacientes EPOC

\section{Objetivo principal}

Describir los efectos sobre la tolerancia al ejercicio, de tres programas de Rehabilitación Respiratoria aplicados a pacientes EPOC y evaluar su evolución tras un período de seguimiento sin estrategias de mantenimiento.

\section{Objetivos secundarios}

- Describir el efecto de los programas sobre la disnea basal y su evolución durante el período de seguimiento.

- Observar los cambios producidos durante el período de seguimiento, en el número de exacerbaciones y las horas de ejercicio físico semanal que realizaban los sujetos.

- Evaluar los efectos sobre la fuerza muscular respiratoria en los sujetos que recibieron un programa que incluía entrenamiento específico de los músculos respiratorios, así como su evolución durante el período de seguimiento.

- Determinar las diferencias en los resultados observados sobre la tolerancia al ejercicio en función del sexo, la edad, la severidad de la EPOC, el IMC, el nivel de actividad física habitual, el número de exacerbaciones, el hábito tabáquico, el uso de $\mathrm{OCD}$, los cambios en la función pulmonar durante el período de seguimiento, el nivel de incremento en la distancia de un 6MWT alcanzado tras el entrenamiento (clínicamente significativo o no) y el tiempo transcurrido en el período de seguimiento.

- Analizar el grado de influencia de distintos factores sobre el deterioro en la tolerancia al ejercicio tras el período de seguimiento.

\section{Objetivo 2}

Evaluar los efectos a corto, medio y largo plazo, de un programa de entrenamiento autoadministrado con incremento progresivo de los niveles de actividad física guiado mediante la 
utilización de un podómetro, en pacientes EPOC. Analizar la influencia de la modificación en el nivel de AF de estos pacientes sobre su estado funcional.

Este objetivo responde a la necesidad de aportar evidencia al conocimiento actual sobre los programas de RR autoadministrada y las estrategias para aumentar el nivel de AF en EPOC. Como se ha indicado anteriormente, es necesario establecer estrategias sencillas y eficaces, de fácil implementación y que consigan la adhesión de los pacientes para prolongar la duración de los efectos.

Para la consecución de este objetivo se ha diseñado un ensayo clínico aleatorizado (ECA) en el que se analiza la eficacia de un programa de entrenamiento autónomo sencillo, al que se le añade un plan de incremento de actividad. De tal modo que la intervención contempla los dos aspectos básicos que se han descrito para aumentar el nivel de AF, ejercicio físico y modificación de los hábitos diarios.

El plan de incremento de actividad se basó en un programa de modificación de los hábitos de los pacientes, puesto que como se ha referido anteriormente, parece que las intervenciones dirigidas a provocar cambios comportamentales son las más eficaces, tanto para los pacientes EPOC como para otras enfermedades crónicas e incluso, para la población general. En el momento del diseño del programa experimental no se había publicado esta evidencia relativa a la EPOC, por lo que nuestro planteamiento se basó en las publicaciones relativas a los individuos sanos.

En el programa se emplea como herramienta básica de retroalimentación y control, un podómetro, puesto que son dispositivos de fácil manejo, especialmente para individuos de edad avanzada como es el grueso de la población EPOC, y cuya efectividad se ha demostrado en estudios anteriores. No obstante, como se ha indicado, la evidencia al respecto es insuficiente, especialmente en cuanto a la duración de los efectos.

Además, en el estudio se analiza la influencia de la modificación del nivel de AF en pacientes EPOC sobre determinadas variables funcionales, puesto que es un aspecto también que requiere mayor investigación.

La metodología y resultados de este estudio se describen en la Parte III de esta tesis. Aquí se indican su hipótesis y objetivos.

\section{Estudio 2}

Eficacia de un programa de entrenamiento autoadministrado e incremento de los niveles de actividad física guiado mediante podómetro en pacientes con EPOC

\section{Hipótesis}

Un programa de entrenamiento autoadministrado e incremento de la actividad física guiado mediante podómetro, aplicado a sujetos con EPOC estable y sedentarios, aumenta la tolerncia alesfuerzo y los niveles de actividad física, a corto y largo plazo, en mayor medida que las recomendaciones aisladas de ejercicio.

\section{Objetivo principal}

Comparar la eficacia a corto, medio y largo plazo, de un programa de entrenamiento autoadministrado e incremento de la actividad física guiado mediante podómetro, frente a recomendaciones aisladas de ejercicio, sobre la tolerancia al esfuerzo y los niveles de actividad en sujetos con EPOC estable y sedentarios. 


\section{Objetivos secundarios}

- Comparar los efectos de ambas intervenciones (programa experimental frente a recomendaciones) a corto, medio y largo plazo, sobre la disnea basal y la calidad de vida.

- Comparar el efecto de ambas intervenciones sobre el número de exacerbaciones aparecidas durante los doce meses siguientes a la finalización del programa.

- Evaluar las diferencias en los resultados en función del sexo, la edad, la severidad, el uso de OCD y el período estacional en el que los sujetos participaron en el estudio.

- Analizar la asociación entre la modificación del nivel de actividad física y los cambios en la capacidad de ejercicio, la calidad de vida, la disnea basal y las exacerbaciones. 
PARTE II

ESTUDIO 1

\section{EFECTOS A CORTO Y LARGO PLAZO DE TRES MODALIDADES DE PROGRAMAS CLÍNICOS DE REHABILITACIÓN RESPIRATORIA EN PACIENTES EPOC}

\section{MATERIAL Y MÉTODOS}

\section{Diseño del estudio}

Estudio observacional longitudinal retrospectivo, en el que se analizan los efectos de tres programas clínicos de Rehabilitación Respiratoria que fueron aplicados a pacientes EPOC en la Escuela Universitaria de Fisioterapia de la ONCE (Universidad Autónoma de Madrid), y la duración de estos efectos a lo largo del tiempo.

\section{Población de estudio}

La muestra se ha obtenido del grupo de pacientes que realizaron un PRR en la Escuela Universitaria de Fisioterapia de la ONCE, entre octubre de 2010 y mayo de 2012, y que acudieron nuevamente al Centro para repetir el programa, tras un período de seguimiento sin intervención, de entre 9 y 18 meses.

Dentro de este grupo se han analizado exclusivamente a los pacientes que presentaban

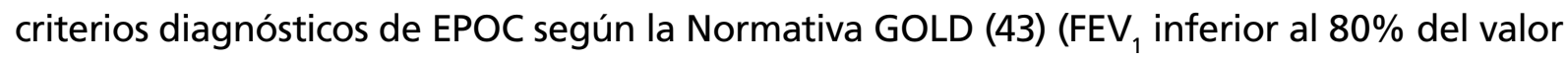
predictivo, así como una relación $\mathrm{FEV}_{1} / \mathrm{FVC}$ menor a 0,7 , evaluados en espirometría forzada). Todos los sujetos debían encontrarse en una situación estable de su enfermedad, con un tratamiento farmacológico adecuadamente pautado y controlado, independientemente de su edad o nivel de gravedad.

Con el fin de reducir los posibles factores de confusión, se han excluido a los sujetos que:

- Presentasen enfermedades neurológicas y/o del aparato locomotor que supusieran la necesidad de incluir cualquier tipo de modificación del programa de entrenamiento en relación al programa general.

- Estuvieran diagnosticados de enfermedad cardiovascular concomitante, a excepción de la hipertensión arterial.

- No cumplieran todas las pautas del programa de entrenamiento (número de sesiones, la duración de las mismas, la progresión de la intensidad del entrenamiento o las características del ejercicio), independientemente de cuál fuera su causa.

- Interrumpieran el programa de entrenamiento.

- Recibieran otro PRR en el período de seguimiento. 


\section{Muestreo y cálculo del tamaño muestral}

Para la conformación de la muestra se ha utilizado un muestreo no probabilístico, de tipo intencional (incluyendo en el análisis a la totalidad de los pacientes que cumplieran los criterios antes descritos), alcanzando de este modo un tamaño muestral de 35 sujetos.

Se realizó un cálculo del tamaño muestral para la estimación de medias en muestras finitas, acotando a la población EPOC residente en Madrid, tomando como variable principal la tolerancia al ejercicio evaluada mediante la distancia alcanzada en una Prueba de Marcha de Seis Minutos y considerando 30 metros como la diferencia mínima clínicamente importante (MCID) (249), con un nivel de confianza del 95\%, una precisión de \pm 3 unidades y con un porcentaje de reposición del $0 \%$. Se obtuvo que una muestra de 255 sujetos hubiera sido suficiente.

Al tratarse de un estudio realizado a posteriori de la obtención de los datos que se analizan y limitado a una población muy pequeña y acotada, la muestra no se ajusta al tamaño muestral óptimo.

\section{Variables}

A todos los pacientes tratados en los PRR de la Escuela Universitaria de Fisioterapia de la ONCE se les realizaba una exploración físico-funcional previa al inicio del tratamiento y otra al finalizar el mismo. Esta evaluación era llevada a cabo por los fisioterapeutas responsables de los programas, siguiendo un protocolo de evaluación y registro consensuado previamente por todo el equipo. Los datos recogidos se almacenaban en la historia clínica de cada paciente, en formato electrónico.

Por otro lado, durante el desarrollo de los programas de entrenamiento se anotaron datos relativos a la evaluación individual de los pacientes en cada sesión, que se archivaron igualmente como anexos a su historia clínica electrónica. Este registro nos ha permitido conocer qué pacientes habían cumplido en su totalidad los PRR y cuáles no, siendo de gran utilidad para la selección de los sujetos de la muestra.

Las variables que se han utilizado en el análisis se han extraído a partir de las historias clínicas de los sujetos de estudio. Los períodos de registro y la distribución de variables en cada período se muestran en la Tabla 2. A continuación, se describe el procedimiento de medición.

\subsection{Variables de caracterización de la muestra}

Para la caracterización de la muestra, se han analizado las siguientes variables sociodemográficas y clínicas:

- Sexo.

- Edad.

- Evolución de la enfermedad: años transcurridos desde el diagnóstico.

- IMC, en $\mathrm{Kg} / \mathrm{m}^{2}$.

- Hábito tabáquico (exfumador/no fumador), años sin fumar y valor de paquetes/año en los sujetos exfumadores.

- Uso de OCD (Sí/No).

- Nivel de severidad según clasificación GOLD (43) (Anexo I).

- Puntuación en el Índice BODE (44) (Anexo I). 
Tabla 2. Distribución temporal de las variables analizadas.

\begin{tabular}{|c|c|c|}
\hline Registro & Temporalidad & Variables \\
\hline Pre-Intervención & $\begin{array}{c}\text { Semana previa al inicio del } \\
\text { primer PRR }\end{array}$ & $\begin{array}{l}\text { Variables de caracterización de la muestra. } \\
\text { Función pulmonar (espirometría forzada). } \\
\text { Número de exacerbaciones en los } 12 \text { meses anteriores. } \\
\text { Tolerancia al ejercicio (Prueba de Marcha de Seis Minutos) } \\
\text { Disnea basal (Escala Modificada del Medica Research Council). } \\
\text { Fuerza muscular respiratoria (Presiones inspiratoria y espiratoria } \\
\text { máximas).. } \\
\text { Nivel de actividad física (horas de ejercicio físico semanal). }\end{array}$ \\
\hline Post-Intervención & $\begin{array}{l}\text { Semanas posterior a la } \\
\text { finalización del PRR (10 } \\
\text { semas tras la medición } \\
\text { pre-intervención) }\end{array}$ & $\begin{array}{l}\text { Tolerancia al ejercicio. } \\
\text { Disnea basal. } \\
\text { Fuerza muscular respiratoria. }\end{array}$ \\
\hline Post-Seguimiento & $\begin{array}{l}\text { Semana previa al inicio } \\
\text { del segundo PRR (tras un } \\
\text { período sin intervención } \\
\text { de entre } 9 \text { y } 18 \text { meses tras } \\
\text { la finalización del primer } \\
\text { PRR) }\end{array}$ & $\begin{array}{l}\text { Duración del período de seguimiento. } \\
\text { Función pulmonar. } \\
\text { Número de exacerbaciones en el período de seguimiento.. } \\
\text { Tolerancia al ejercicio. } \\
\text { Disnea basal. } \\
\text { Fuerza muscular respiratoria. } \\
\text { Nivel de actividad física. }\end{array}$ \\
\hline
\end{tabular}

La tabla muestra las variables analizadas en cada uno de los tres momentos de registro.

\subsection{Función pulmonar y número de exacerbaciones ${ }^{1}$}

A todos los pacientes se les realizó una espirometría forzada, previamente a comenzar los programas y tras el período de seguimiento, mediante el espirómetro modelo MicroLab (Micromedical; Kent, UK). La prueba se efectuó siguiendo los principios de aplicación descritos en el Manual de Procedimientos SEPAR publicado en 2002 (250) y coincidentes con la Normativa SEPAR publicada en 2013, que constituye una revisión del procedimiento de espirometría (251).

Para este estudio se analizaron las siguientes variables relativas a la función pulmonar: $\mathrm{FEV}_{1}$, $\mathrm{FEV}_{1}(\%), \mathrm{FVC}$ (capacidad vital forzada), FVC (\%) y relación $\mathrm{FEV}_{1} / \mathrm{FVC}$.

En cuanto a exacerbaciones, se anotaron el número de episodios sufridos en los 12 meses previos al inicio del primer programa y durante el período de seguimiento. Se consideraba exacerbación al empeoramiento en al menos dos de los síntomas habituales (disnea, cantidad de expectoración y color del esputo) durante más de 24 horas (252), con necesidad de tratamiento antibiótico y/o ingreso hospitalario.

\subsection{Tolerancia al ejercicio}

La tolerancia al ejercicio fue evaluada mediante la Prueba de Marcha de Seis Minutos. Ésta es una prueba de esfuerzo submáxima que "nos permite evaluar de forma global e integrada las diferentes funciones (cardíaca, respiratoria, transporte periférico de oxígeno, bioenergética muscular e integración neuromuscular) que determinan la capacidad aeróbica del

(1) El análisis pre-intervención de estas variables se utilizó también para la caracterización de la muestra.

El registro post-seguimiento se analizó para descartar el posible deterioro en la función pulmonar

como factor de influencia en los cambios en las variables resultado tras el seguimiento. 
paciente" (48). Es de fácil realización y está ampliamente estandarizada. La Normativa SEPAR sobre Disfunción Muscular en EPOC de 2015 la ha definido como: "el protocolo simple de ejercicio de referencia en la EPOC, de gran utilidad para determinar el estado funcional de los sujetos y medir el efecto de las intervenciones, así como la herramienta más empleada para evaluar la capacidad aeróbica en la práctica clínica" (48).

En la prueba, el sujeto debe caminar en un circuito recto y sin irregularidades, de al menos 30 metros, durante un tiempo de 6 minutos, con el objetivo de alcanzar la máxima distancia posible en dicho período (se debe caminar lo más deprisa posible, sin llegar a correr). A lo largo de la prueba, el individuo puede modificar su ritmo e incluso pararse, en cuyo caso no se detiene el cronómetro, pero se contabiliza el tiempo total de descanso.

En los sujetos de la muestra, esta prueba se realizó siguiendo el protocolo establecido por la ATS en 2002 (115), utilizando un pasillo de 30 metros de longitud. Este protocolo es coherente con la última actualización de la ATS publicada en 2014 (249).

En cada prueba se recogieron las siguientes variables:

- Distancia total recorrida en metros.

- Variación durante la prueba (post vs pre) en la disnea percibida y en la fatiga deMMII, según la Escala de Borg Modificada (253).

- Variación en la frecuencia cardiaca (FC) durante la prueba (post vs pre).

- Variación en la saturación de oxígeno $\left(\mathrm{SaO}_{2}\right)$ durante la prueba (post vs pre). Este parámetro y el anterior se midieron mediante un pulsioxímetro modelo Nonin-8500 (Nonin Medical; Minesota, EEUU).

Cada sujeto debía efectuar dos pruebas con 30 minutos de descanso entre ambas, quedando registrados en la historia clínica, sólo los datos del test con mejores resultados.

\subsection{Disnea basal}

Para evaluarla se utilizó la Escala Modificada del Medical Research Council (mMRC) (254). Se trata de una escala numérica de cinco niveles (del 0 al 4), cada uno de los cuales se corresponde con una definición breve de la sensación de disnea percibida. El sujeto debe indicar al entrevistador, a qué nivel se asemeja más su sensación de disnea habitual.

\subsection{Fuerza muscular respiratoria}

Esta variable se evaluó sólo en los sujetos que recibieron el programa que incluía entrenamiento de los músculos respiratorios. Para ello se midió la presión inspiratoria máxima (PIM) y la presión espiratoria máxima (PEM), utilizando el dispositivo medidor de presiones respiratorias MicroRPM (CareFusion; Kent, UK), calibrado en $\mathrm{cmH}_{2} \mathrm{O}$.

El procedimiento de medición utilizado seguía las directrices descritas por SEPAR en su Manual de Procedimientos de Función Pulmonar II de 2004 (255).

Para el registro de la PIM, los pacientes debían realizar una inspiración máxima y a alto flujo a través del medidor, desde volumen residual. Para el registro de la PEM, los pacientes debían realizar una espiración máxima y a alto flujo, partiendo de capacidad pulmonar total.

Cada maniobra de PIM o PEM se repetía tres veces, registrándose el valor más alto. Los resultados obtenidos se expresaron en valores absolutos y en porcentaje del valor teórico, según la ecuación descrita por Morales (256). 


\subsection{Nivel de actividad física}

El nivel de actividad física de cada sujeto se evaluó recogiendo el número de horas semanales que indicaban realizar ejercicio al menos, de intensidad moderada (caminar, bicicleta, nadar, bailar, gimnasia de mantenimiento, correr, deporte de tipo aeróbico, actividades de jardinería, etc.) y siempre que se llevase a cabo de forma habitual (al menos todas las semanas durante tres meses consecutivos). No se contempló el tiempo que los sujetos indicaban que dedicaban a pasear o a realizar actividades domésticas suaves.

\section{Intervención: Programas de Rehabilitación Respiratoria aplicados}

Los sujetos incluidos en el estudio recibieron uno de los tres PRR que se estaban desarrollando en la Escuela Universitaria de Fisioterapia de la ONCE en el período analizado. El que cada sujeto recibiera un programa u otro dependió de la época de ingreso, puesto que cada programa se utilizó en el Centro para el tratamiento de los pacientes respiratorios crónicos en un momento determinado, sustituyendo cada protocolo al anterior. Estos PRR fueron:

- PRR 1: Entrenamiento de resistencia global.

- PRR 2: Entrenamiento de resistencia global + entrenamiento de fuerza de miembros superiores e inferiores.

- PRR 3: Entrenamiento de resistencia global + entrenamiento de fuerza de miembros superiores e inferiores + entrenamiento de músculos respiratorios.

Cada uno de estos tres programas tuvieron una duración de ocho semanas consecutivas y fueron dirigidos y supervisados por fisioterapeutas especializados en el campo de la Rehabilitación Respiratoria, que seguían un protocolo general previamente diseñado y consensuado por el equipo, en cuanto a modalidad de ejercicio, progresión del entrenamiento en tiempo e intensidad y monitorización de los pacientes. Estos protocolos, si bien, presentaban unas directrices generales, permitían la adaptación a las necesidades individuales de cada paciente, a criterio del fisioterapeuta responsable.

Para el entrenamiento físico se siguieron en todos los programas, las pautas generales establecidas por la Guía de Práctica Clínica para Fisioterapeutas publicada por Langer y col. en 2009 (78) y la de ATS/ERS de 2006 (47). Además, se ha comprobado que los protocolos fueron acordes con las actualizaciones efectuadas por estas Sociedades en 2013 (72) y 2014 (49), así como con la Guía de la British Thoracic Society (BTS) (83) y las recientes Normativas SEPAR $(48,81)$.

Todos los programas se ejecutaron de manera grupal, en grupos de cuatro pacientes, con características similares en su enfermedad y edad. No se tuvo en cuenta el sexo para organizar los grupos de entrenamiento, de tal modo que hubo grupos mixtos y otros sólo de hombres o de mujeres.

A continuación, se describen los protocolos de forma detallada. En la Tabla 3 se presenta un esquema general de los mismos.

\subsection{Descripción de los programas de entrenamiento: PRR 1}

Se efectuaban tres sesiones semanales, de una duración aproximada de 1 a 1,5 horas. Cada sesión se dividía en dos partes:

- Primera parte: técnicas de Fisioterapia Rrspiratoria y educación sanitaria.

- Segunda parte: entrenamiento de resistencia global. 


\section{Primera parte}

Se llevaban a cabo técnicas de Fisioterapia Respiratoria, tales como:

- Reeducación ventilatoria (Figuras 6, 7, 8, 9 y 10): Ventilación dirigida diafragmática y costal (257), ventilación con labios fruncidos (258), etc. El objetivo era optimizar la ventilación de los pacientes e instruirles en el empleo habitual de un patrón ventilatorio eficaz, reduciendo el atrapamiento aéreo (259). Las técnicas empleadas en cada caso dependían de la situación clínica de cada paciente, evitando el adiestramiento diafragmático en pacientes con hiperinsuflación importante, tal y como indican las guías de práctica clínica $(78,81,260)$. No se consideró la influencia de estas técnicas en los resultados de los programas, ya que no se ha demostrado que su aplicación en el contexto de la RR produzca ningún efecto sobre la capacidad de ejercicio, la disnea o la calidad de vida (261).

- Drenaje bronquial (Figuras 11, 12, 13 y 14): Se llevaban a cabo técnicas como la Espiración Lenta Total con Glotis Abierta en Decúbito Lateral (ELTGOL) (262) y las Técnicas de Espiración Forzada (TEF) (263), así como el adiestramiento de una tos

Tabla 3. Esquema-resumen de los programas de Rehabilitación Respiratoria aplicados.

\begin{tabular}{|c|c|c|}
\hline \multicolumn{3}{|c|}{ Aspectos generales } \\
\hline \multirow{3}{*}{\multicolumn{3}{|c|}{$\begin{array}{l}8 \text { semanas } \\
1 \mathrm{~h} \text {-1h30min por sesión } \\
\text { Grupos de } 4 \text { pacientes }\end{array}$}} \\
\hline & & \\
\hline & & \\
\hline PRR 1 & PRR2 & PRR3 \\
\hline $\begin{array}{l}\text { - } 3 \text { sesiones semanales. } \\
\text { - Educación sanitaria. } \\
\text { - Fisioterapia Respiratoria: } \\
\text { técnicas de ventilación, manejo de } \\
\text { secreciones, cinesiterapia del tórax. }\end{array}$ & \multirow[t]{2}{*}{$\begin{array}{c}\text { PRR } 1 \\
+\end{array}$} & \multirow{6}{*}{$\begin{array}{c}\text { PRR } 2 \\
+\end{array}$} \\
\hline \multirow{6}{*}{$\begin{array}{l}\text { - Entrenamiento de resistencia } \\
\text { global de tipo interválico en } \\
\text { cicloergómetro y bicicleta estática } \\
\text { (ver Tabla 4a). }\end{array}$} & & \\
\hline & - 2 sesiones semanales. & \\
\hline & & \\
\hline & $\begin{array}{l}\text { Entrenamiento de MMII: } \\
\text { cuádriceps e isquiotibiales (banco } \\
\text { de cuádriceps). }\end{array}$ & \\
\hline & $\begin{array}{l}\text { - Entrenamiento de MMSS: } \\
\text { bíceps braquial, tríceps braquial y } \\
\text { musculatura del hombro mediante } \\
\text { mancuernas. }\end{array}$ & \\
\hline & & $\begin{array}{l}\text { - } 5 \text { sesiones semanales en domicilio. } \\
\text { - Entrenamiento específico de los } \\
\text { músculos respiratorios mediante } \\
\text { válvula de tipo umbral (ver Tabla 4c). }\end{array}$ \\
\hline
\end{tabular}

Esta tabla muestra los componentes generales de los tres programas de Rehabilitación Respiratoria aplicados a los sujetos de estudio. 

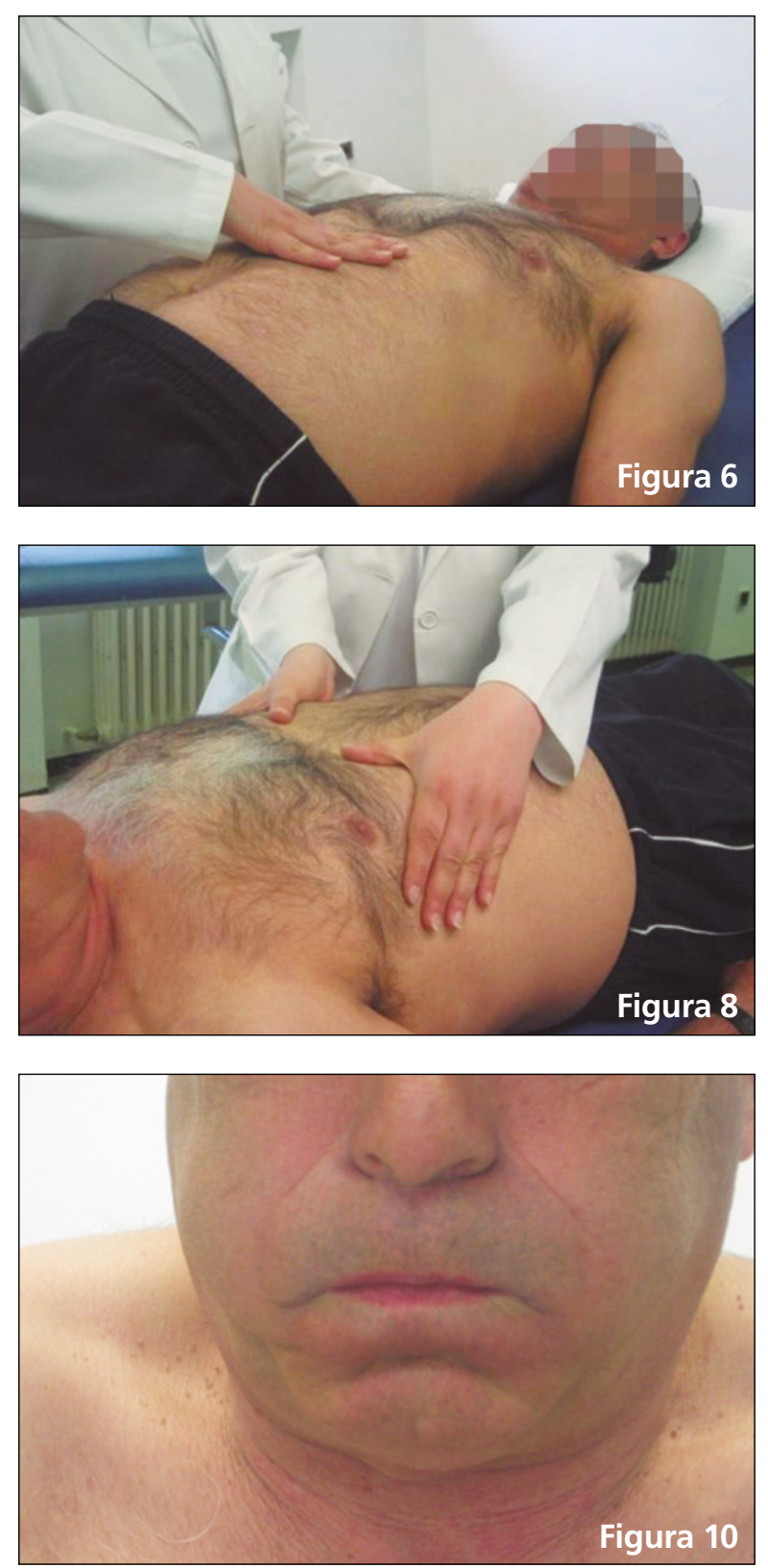
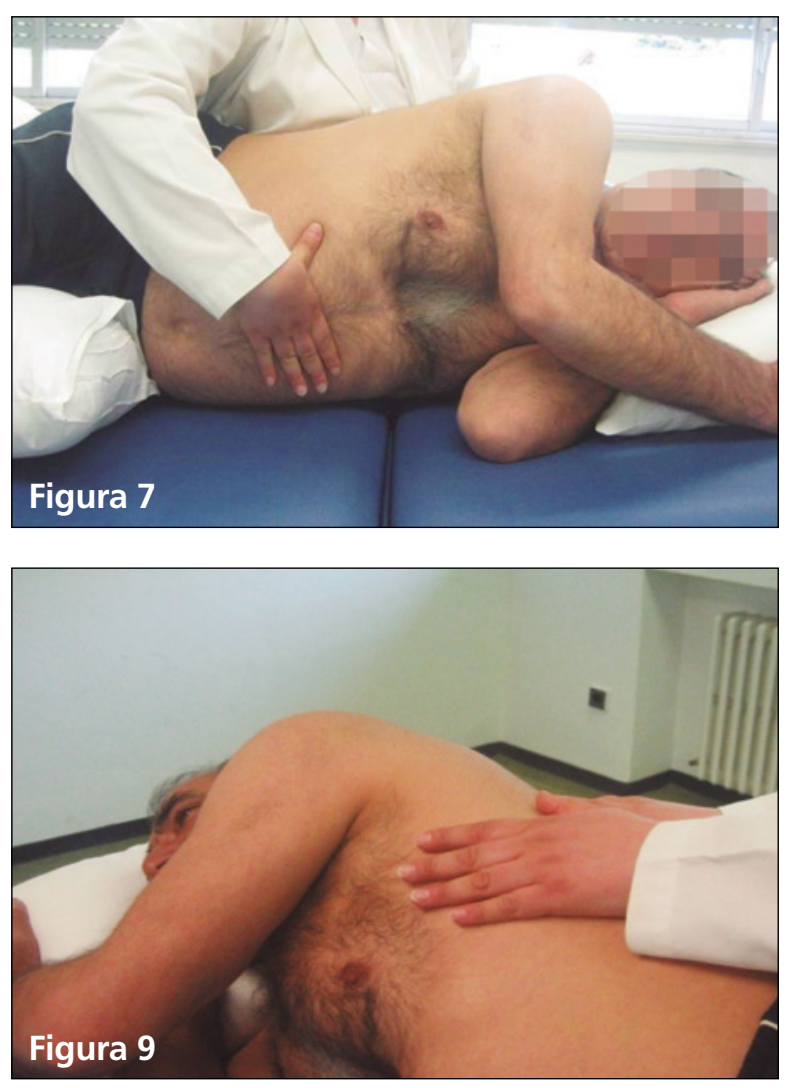

Figura 6. Técnica de ventilación dirigida diafragmática en posición de decúbito supino.

Figura 7. Técnica de ventilación dirigida diafragmática en posición de decúbito lateral.

Figura 8. Técnica de ventilación dirigida costal basal bilateral.

Figura 9. Técnica de ventilación dirigida costal basal unilateral.

Figura 10. Ventilación con "labios fruncidos".

eficaz. Si era preciso, se aplicaba nebulización con suero salino e incluso, en pacientes hipersecretantes se les adiestraba en el empleo de ayudas técnicas para domicilio, como el Flutter® VRP1 (Medtronic GmbH; Berlín, Alemania) y la A-Capella® (Smitchs MedicalADS, Inc.; Rockland, EEUU) $(264,265)$. La decisión de emplear unas técnicas u otras o ninguna de ellas, venía determinada por los hallazgos de una auscultación pulmonar previa, que se practicaba al inicio de la sesión a todos los pacientes.

A pesar de que esta intervención es previa al año 2013, todas las técnicas seguían los principios de aplicación que se describen en el Manual de Procedimientos publicado por SEPAR en 2013 sobre técnicas manuales e instrumentales para el manejo de secreciones bronquiales en el adulto (266).

- Movilización del tórax: Ejercicios de cinesiterapia activa libre del tórax combinados con respiración, con el objetivo de flexibilizar la caja torácica (Figuras 15, 16 y 17).

Así mismo, a lo largo del programa, se intercaló educación sanitaria durante esta primera parte de la sesión. En este sentido, se realizaban charlas dirigidas al manejo de la enfermedad, 

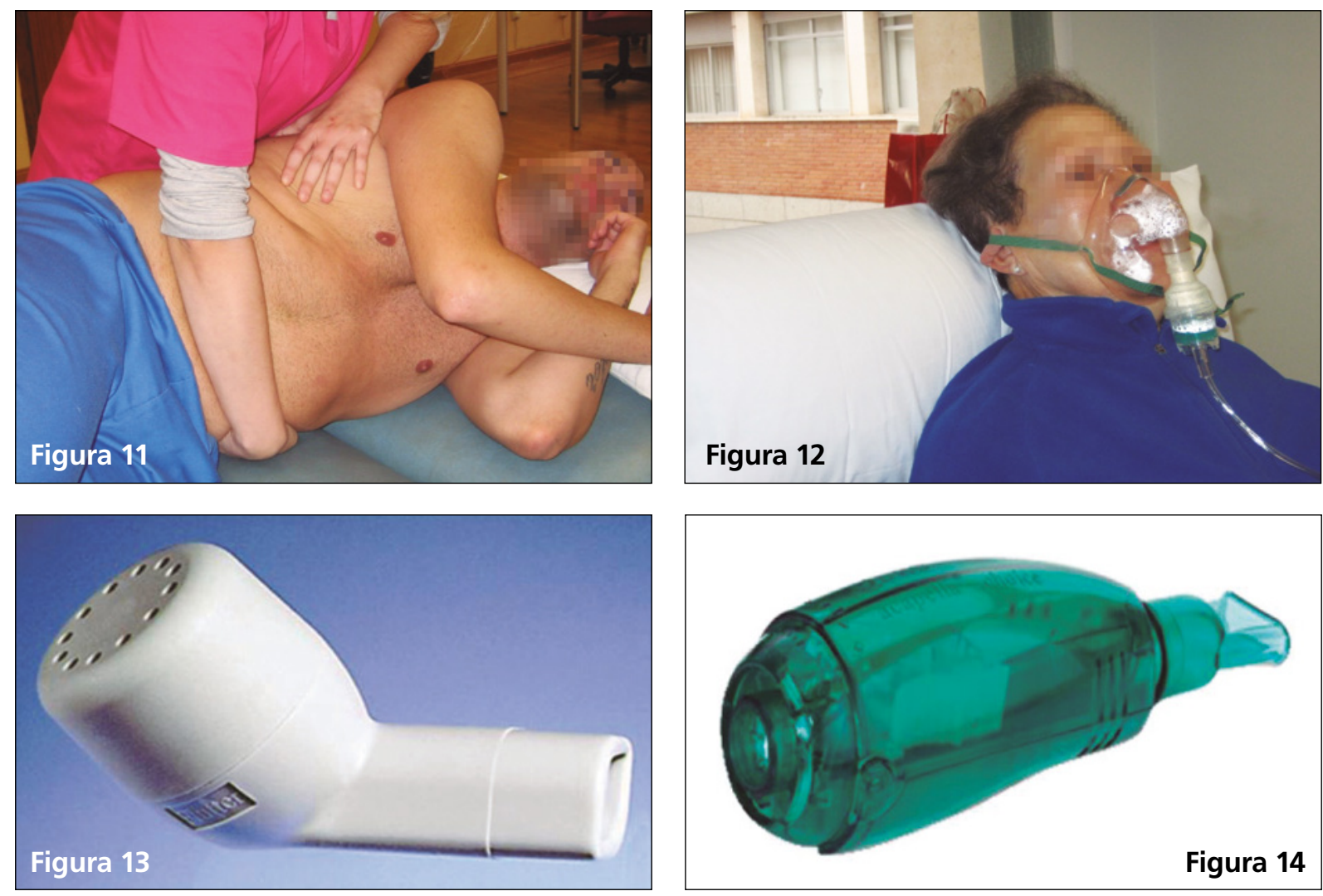

Figura 11. Técnica de ELTGOL. Figura 12. Aplicación de nebulización con suero salino.

Figura 13. Flutter® VRP1. Figura 14. A-Capella®.

identificación de síntomas, uso correcto de los dispositivos de inhalación, estrategias para el control de la disnea, técnicas de ahorro de energía (267), pautas y recomendaciones de vida activa y saludable, etc.

La educación sanitaria efectuada durante los programas siguió las recomendaciones de las principales guías de práctica clínica sobre RR $(47,72,81,83)$.

\section{Segunda parte}

En la segunda parte de la sesión se llevaba a cabo el programa de entrenamiento, que incluía:

- Registro de constantes iniciales de los pacientes.

- Calentamiento.

- Entrenamiento propiamente dicho.

- Enfriamiento.

- Registro de constantes finales de cada paciente.

El registro inicial y final de constantes permitía conocer la evolución de cada paciente y el impacto del ejercicio. Se medía:

- Tensión arterial (TA).

- FC.

- $\mathrm{SaO}_{2}$.

- Disnea percibida, según la Escala de Borg Modificada (253).

- Fatiga en los miembros inferiores según la Escala de Borg Modificada (253). 
Del mismo modo, las medidas iniciales permitían determinar si el paciente podía entrenar ese día y en qué condiciones. Por ejemplo, una TA superior a $180-120 \mathrm{mmHg}$ y una FC superior a 120 latidos por minuto (lpm), determinaban que ese paciente no pudiera entrenar.

En el calentamiento se dedicaban unos pocos minutos a realizar una tabla general de flexibilización y estiramiento de los MMII (especialmente cuádriceps e isquiotibiales). Por su parte, al final del entrenamiento, en el enfriamiento, se repetían los ejercicios de estiramiento muscular (Figuras 18 y 19).

En cuanto al programa de entrenamiento de resistencia, se aplicó un protocolo de tipo interválico ya que, según las principales guías de práctica clínica (47$49,72,78,81,83)$, esta modalidad reduce la disnea y la fatiga y por tanto, es más tolerable por los pacientes. Durante las tres primeras semanas del programa, se utilizaba un cicloergómetro y durante el resto, se combinaba el ejercicio en cicloergómetro con entrenamiento en bicicleta elíptica (Figuras 20 y 21).

A lo largo de las ocho semanas se iban incrementando tanto el tiempo como la intensidad del entrenamiento, según un protocolo preestablecido. No obstante, como ya se ha indicado antes, este protocolo se podía modificar ligeramente para adaptarse a las necesidades de cada paciente, en el caso de que éstos no pudieran seguir el ritmo de la progresión. En la Tabla 4a se resume la progresión del programa.

Para la determinación de la carga de trabajo durante el entrenamiento, puesto que todos los pacientes carecían de una prueba de esfuerzo cardiopulmonar previa, se utilizaron los resultados obtenidos en un IST que se realizaba antes de comenzar el programa. Ésta es una prueba de marcha, que ha sido validada para población EPOC (268) y que presenta una fuerte correlación con el $\mathrm{VO}_{2} \max (269)$. En 2006 se publicó una ecuación que permite calcular el trabajo máximo alcanzado (Wmax) en función del resul-
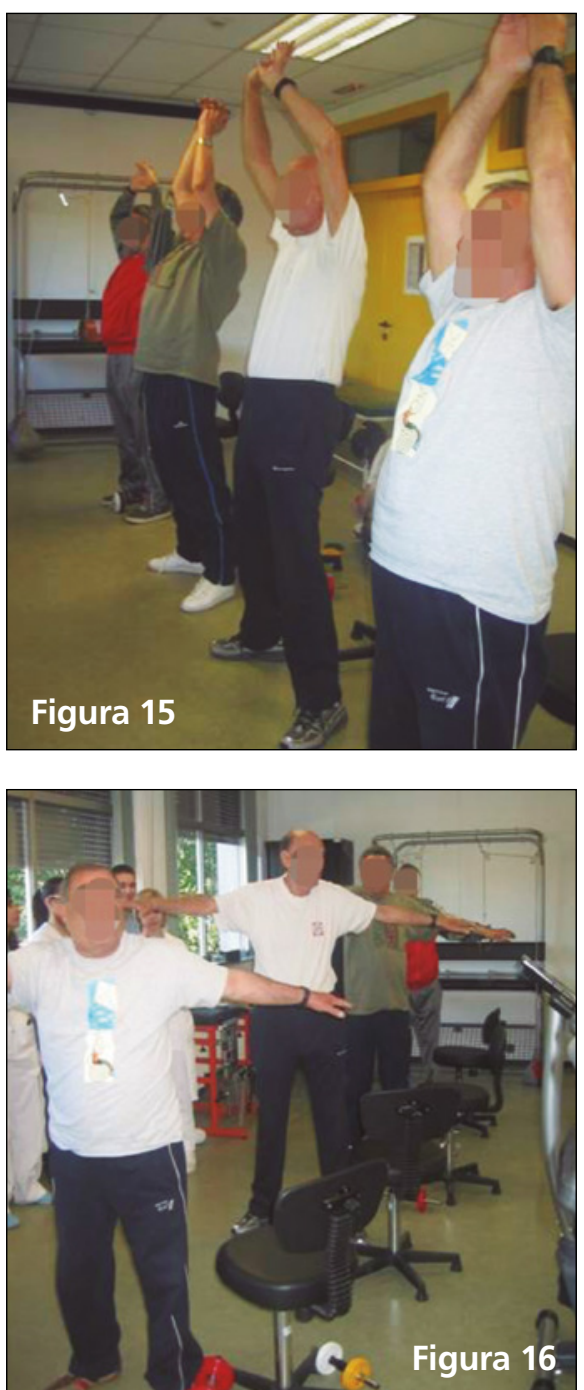
tado de un IST (270). Esta ecuación, que se utilizaba para obtener el Wmax de cada paciente y a partir de ahí, determinar las cargas de trabajo a aplicar en cada semana del entrenamiento, es la siguiente:

Watts $=(0,0025 \times$ distancia $(\mathrm{m}) \times$ peso $(\mathrm{Kg}))+10,19$

Durante el entrenamiento, a los pacientes se les monitorizaba la $\mathrm{FC}$ y la $\mathrm{SaO}_{2}$ mediante un pulsioxímetro

Figura 15. Ejercicios de flexibilización del tórax en flexo-extensión.

Figura 16. Ejercicios de flexibilización del tórax en rotación.

Figura 17. Ejercicios de flexibilización del tórax en lateralidad.

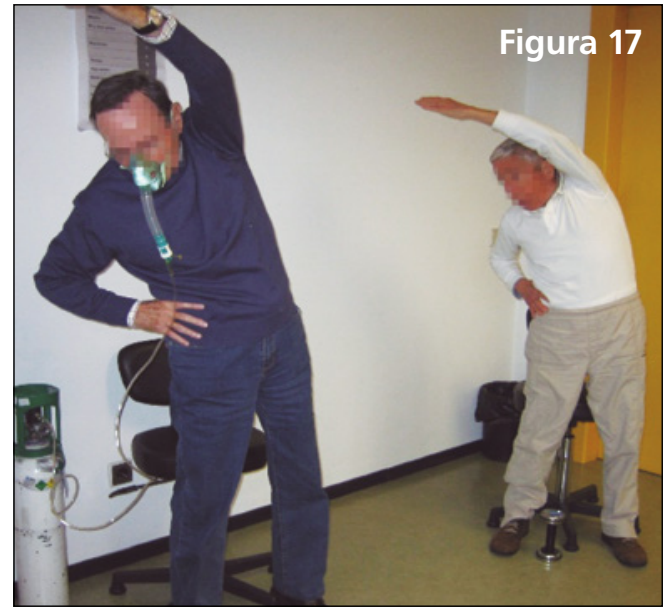



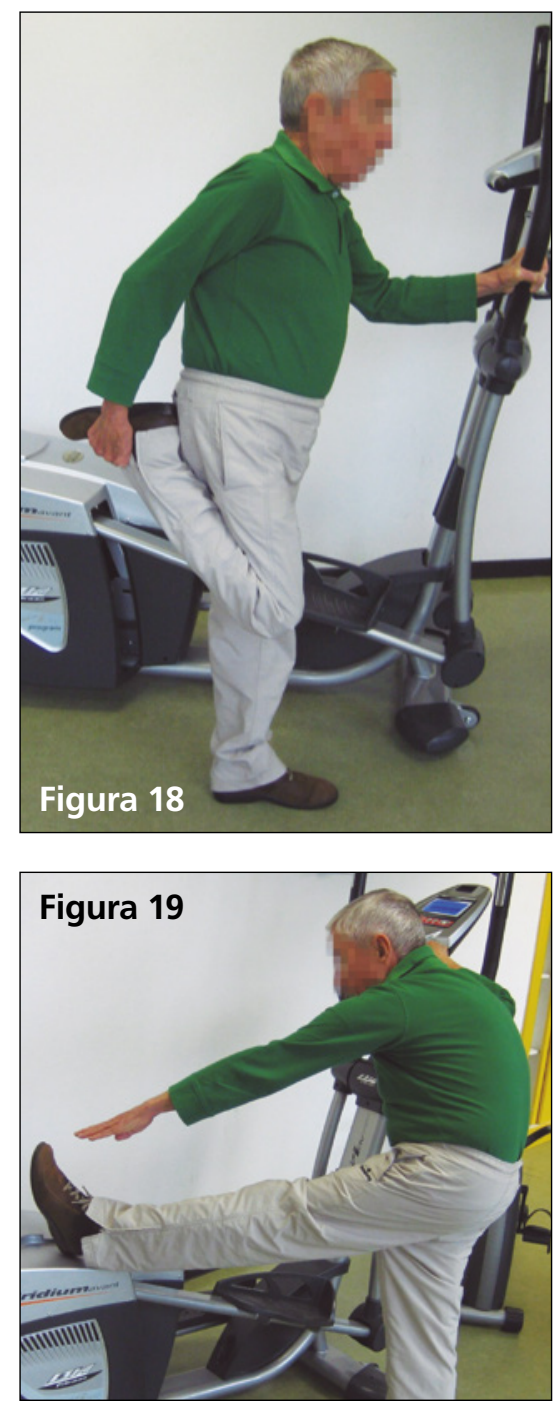

Figura 18. Ejercicios de autoestiramiento del músculo cuádriceps.

Figura 19. Ejercicios de autoestiramiento de los músculos isquiotibiales.

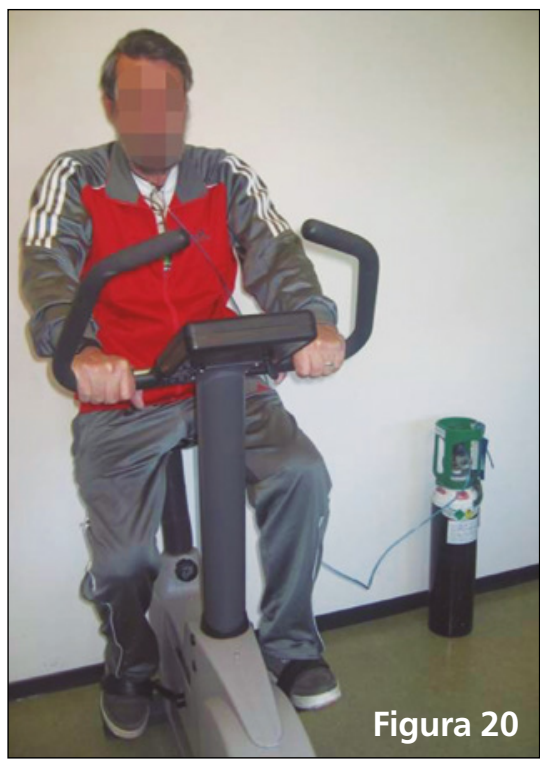

que debían llevar constantemente. Del mismo modo, se vigilaba la aparición de reacciones de tipo vegetativo, dolor precordial, sensación de disnea intensa, etc.

Con el objetivo de optimizar el entrenamiento, éste se llevaba a cabo con oxígeno suplementario en el caso de pacientes con prescripción de oxigenoterapia continua, así como en aquellos en que la $\mathrm{SaO}_{2}$ inicial o durante el ejercicio bajara por debajo del $90 \%$, tal y como recomiendan las guías de práctica clínica $(48,72,78,81,83)$. La concentración aplicada se ajustaba para mantener una $\mathrm{SaO}_{2}$ entre $90-94 \%$.

\subsection{Descripción de los programas de entrenamiento: PRR 2}

En este programa, los pacientes efectuaban cinco sesiones semanales. En tres de ellas realizaban todo exactamente igual a lo descrito para el PRR 1 y en las dos sesiones restantes, se introducía un programa de entrenamiento de fuerza de MMSS y MMII. En estas sesiones de entrenamiento de fuerza se llevaba a cabo:

- Registro inicial de constantes.

- Calentamiento.

- Entrenamiento de fuerza propiamente dicho.

- Enfriamiento.

- Registro final de constantes.

El registro inicial y final de constantes en cada paciente tenía la misma finalidad y se ejecutaba igual que en las sesiones de entrenamiento aeróbico. Así mismo, en el calentamiento inicial se realizaba una pequeña tabla de flexibilización general de los miembros y técnicas de estiramiento de la musculatura a entrenar. Estos estiramientos se repetían al finalizar, en la fase de enfriamiento.

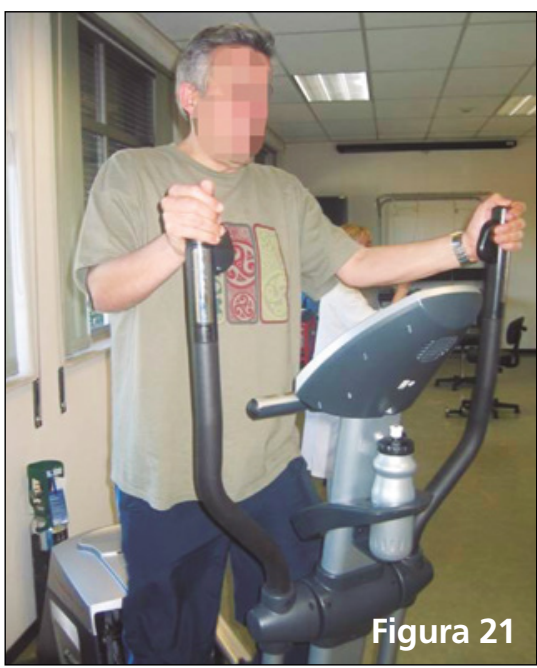

Figura 20. Entrenamiento aeróbico en cicloergómetro. Figura 21. Entrenamiento aeróbico en bicicleta elíptica. 
En el entrenamiento de fuerza se trabajaban específicamente:

- MMII: cuádriceps e isquiotibiales, mediante cargas indirectas en bancos de cuádriceps (Figuras 22 y 23).

- MMSS: bíceps braquial y tríceps braquial de forma analítica. Para la musculatura del hombro se utilizaron patrones globales, siguiendo los principios de la técnica de Facilitación Neuromuscular Propioceptiva (FNP) descrita por Kabat (271), en concreto a través de los patrones de movimiento de primera y segunda diagonal (D1 y D2). La potenciación de MMSS se llevó a cabo mediante cargas directas a través de sistemas de mancuernas de pesos intercambiables (Figuras 24, 25, 26 y 27).

Al igual que en el entrenamiento aeróbico, se estableció previamente un protocolo para la progresión del programa, tanto en número de repeticiones como en carga de trabajo. En la Tabla $4 \mathrm{~b}$ se puede observar este protocolo.

Las cargas de trabajo en cada semana se aplicaron como un porcentaje del 1RM (resistencia máxima que un músculo puede vencer en una única contracción isotónica concéntrica), que se evaluó para cada músculo o grupo muscular a entrenar, previo al inicio del programa de entrenamiento.

Los ejercicios se efectuaban de forma unilateral, alternando uno y otro miembro hasta completar el número de series. A continuación, se pasaba a realizar el entrenamiento de otro músculo o grupo muscular, dejando un período de descanso de 2-3 minutos (hasta la recuperación del paciente), período que se dedicaba a realizar ventilación dirigida.

En cuanto a los aspectos relacionados con el control de los pacientes durante el ejercicio y la aplicación de oxígeno suplementario, se seguían las mismas pautas que para el entrenamiento aeróbico.

\subsection{Descripción de los programas de entrenamiento: PRR 3}

El PRR 3 tenía una estructura y características idénticas a las descritas para el PRR 2, pero añadiendo entrenamiento específico de los músculos respiratorios.

Tabla 4a. Protocolo de entrenamiento de resistencia global de tipo interválico.

\begin{tabular}{|c|c|c|c|c|c|c|}
\hline \multirow{2}{*}{ Semana } & \multicolumn{2}{|c|}{ Períodos pico } & \multicolumn{2}{c|}{ Períodos recuperación } & \multicolumn{2}{c|}{$N^{\circ}$ ciclos } \\
\cline { 2 - 7 } & $\mathrm{T}(\mathbf{m i n})$ & $\%$ Wmax & $\mathrm{T}$ (min) & $\%$ Wmax & Cicloerg. & Elíptica \\
\hline $\mathbf{1}$ & 1 & 70 & 2 & 20 & 15 & 0 \\
\hline $\mathbf{2}$ & 1,5 & 75 & 2 & 30 & 12 & 0 \\
\hline $\mathbf{3}$ & 2 & 80 & 2 & 40 & 11 & 0 \\
\hline $\mathbf{4}$ & 2 & 85 & 1 & 40 & 10 & 5 \\
\hline $\mathbf{5}$ & 2,5 & 90 & 1 & 40 & 8 & 4 \\
\hline $\mathbf{6}$ & 3 & 95 & 1 & 50 & 7 & 4 \\
\hline $\mathbf{7}$ & 3,5 & 100 & 1 & 50 & 6 & 4 \\
\hline $\mathbf{8}$ & 4 & 110 & 1 & 60 & 5 & 5 \\
\hline
\end{tabular}

Esta tabla muestra el protocolo de progresión semana a semana, de los tiempos ( $\mathrm{T}(\mathrm{min})$ ) y las cargas (\% Wmax). Así mismo, se detalla el número de ciclos completos establecidos para cada equipo de entrenamiento (cicloergómetro y bicicleta elíptica). Cada ciclo incluye un período de alta intensidad (período pico) y otro de baja intensidad (período de recuperación). 
En este programa se incluyeron sólo a pacientes que presentaban debilidad en los músculos inspiratorios y/o espiratorios, entrenando un grupo muscular u otro o ambos, dependiendo de dónde presentaran el déficit. Se consideraba que tenían debilidad si el valor de la PIM - la PEM obtenido presentaba una reducción superior al $20 \%$ en relación al valor teórico, calculado mediante la ecuación de Morales (256).

El entrenamiento se realizó con una válvula de tipo umbral que el Centro proporcionó a los pacientes, la válvula Orygendual@ (Forumed, SL; Pals, España) (Figura 28). Este dispositivo permite el trabajo tanto de músculos inspiratorios como espiratorios, ofreciendo una resistencia al esfuerzo ventilatorio del paciente. Esta resistencia puede graduarse en $\mathrm{cmH}_{2} \mathrm{O}$ para establecer y objetivar la progresión del entrenamiento.

Los pacientes debían entrenar con el dispositivo en su domicilio, cinco días en semana en dos períodos cada día. El entrenamiento consistía en alternar un minuto de trabajo ventilatorio (o diez respiraciones) a través de la válvula y un minuto de descanso con ventilación libre, hasta completar 15 minutos de entrenamiento en cada período (30 minutos al día en total). En una de las sesiones semanales en el Centro, se llevaba a cabo la supervisión y adaptación de nuevas cargas.

Básicamente se realizó un entrenamiento submáximo de resistencia, que se combinó con un entrenamiento de fuerza a partir de la tercera semana. En la Tabla 4c se muestra la progresión de las cargas de entrenamiento.

\section{Almacenamiento y análisis de los datos}

Todos los datos extraídos a partir de las historias clínicas de los sujetos de estudio fueron archivados en una hoja de cálculo del programa Microsoft $\circledast$ Excel 2010, elaborada para tal fin y posteriormente, transcritos al programa de análisis estadístico (paquete informático SPSS $®$ Statistics versión 20.0 (SPSS Incorporation; Chicago, EEUU)).

Se llevó a cabo un análisis descriptivo para todas las variables en la muestra completa y segmentada según el tipo de entrenamiento. Para las cualitativas se calcularon las frecuencias absolutas y relativas de cada una de sus categorías. Para las cuantitativas se obtuvieron medidas de tendencia central (media) y de dispersión (desviación estándar), así como el rango. Se analizó la homogeneidad de los grupos al inicio del estudio mediante la prueba Exacta de Fisher (variables cualitativas), ANOVA (o prueba de Welch si $p<0,05$

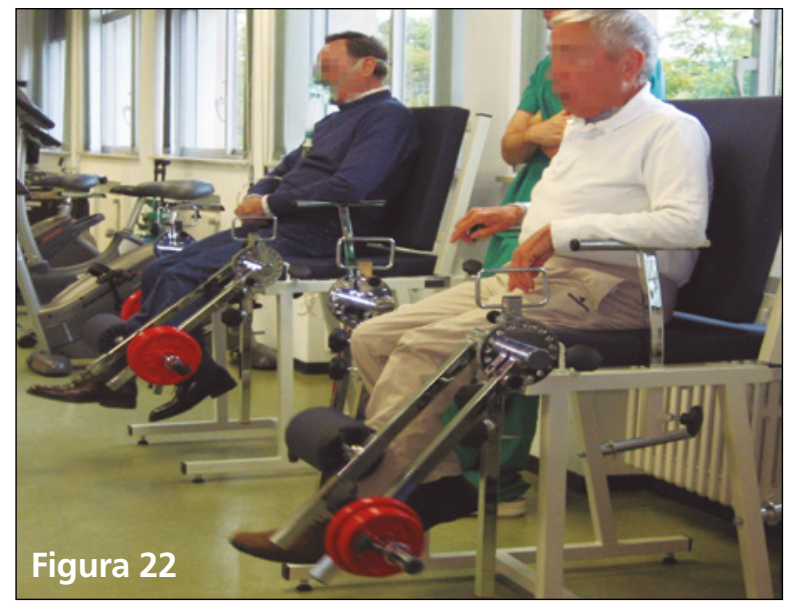

Figura 22. Entrenamiento de fuerza del cuádriceps en el banco de cuádriceps.

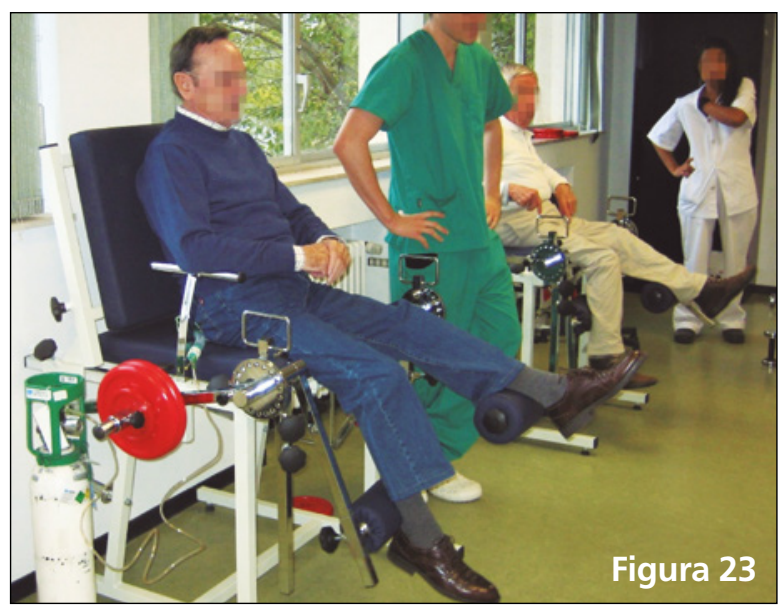

Figura 23. Entrenamiento de fuerza de los músculos isquiotibiales en el banco de cuádriceps. 

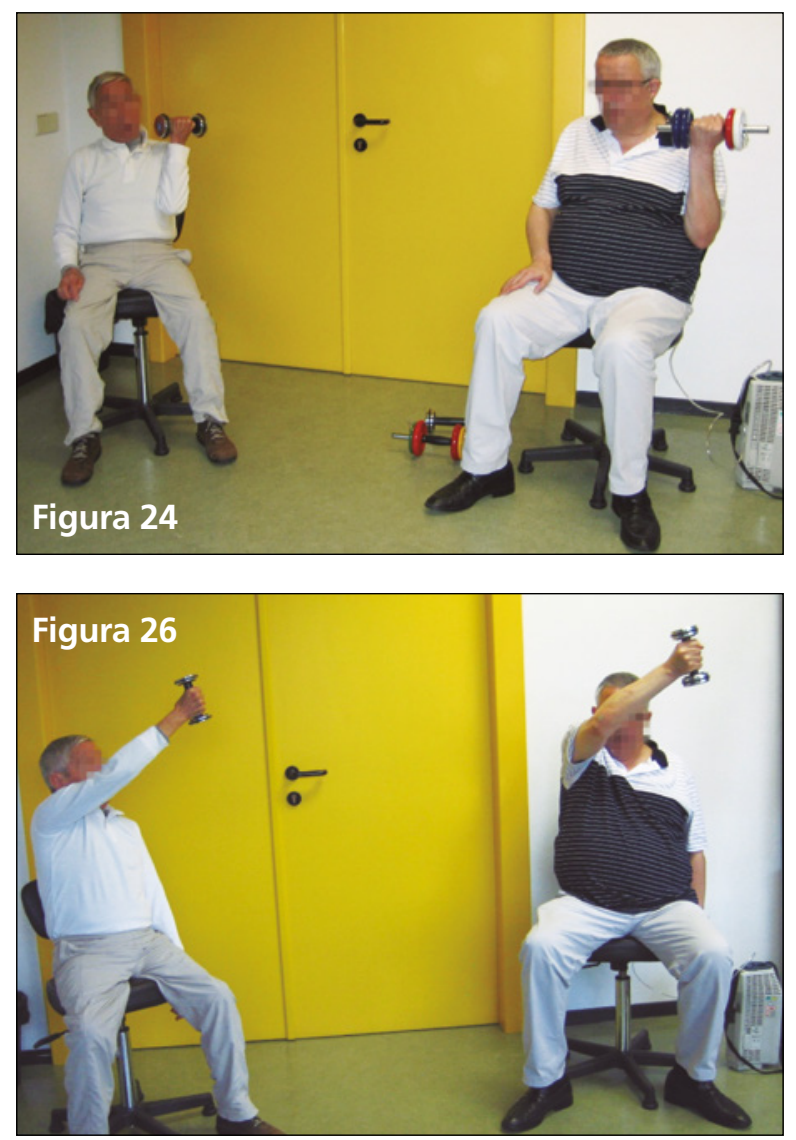

Figura 24. Entrenamiento de fuerza del músculo bíceps braquial.

Figura 26. Ejecución de la primera diagonal de FNP.
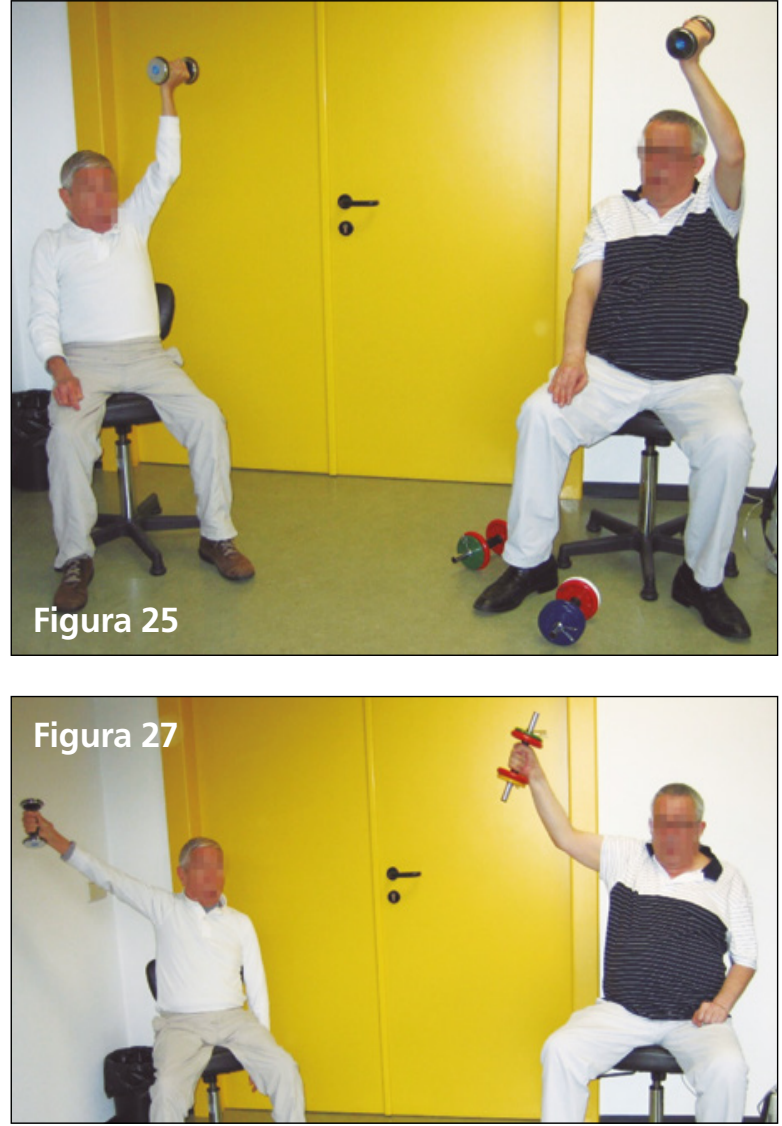

Figura 25. Entrenamiento de fuerza del músculo tríceps braquial.

Figura 27. Ejecución de la segunda diagonal de FNP.

en la prueba de Levene) y de Kruskal-Wallis (variables cuantitativas, según distribución normal o no).

Se realizó un análisis comparativo de los resultados post-entrenamiento vs pre-entrenamiento y post-seguimiento vs post-entrenamiento, para la muestra completa y segmentada según el grupo de PRR, reportando la media, la desviación estándar, el intervalo de confianza al 95\% y el porcentaje de variación observado, así como el valor de $\mathrm{p}$ calculado a partir de pruebas paramétricas ( $T$ para datos pareados) y no paramétricas (Wilcoxon). Así mismo, se analizó la significación estadística de las diferencias intergrupo mediante ANOVA (o prueba de Welch) y la prueba de KruskalWallis, según distribución de la muestra.

Del mismo modo, se realizó un análisis de subgrupos para determinar la existencia de diferencias en los resultados observados en la variable principal (tolerancia al ejercicio) para la muestra completa, según distintos niveles de segmentación: sexo, severidad de la EPOC según GOLD (43) (Grados I-II y Grados III-IV), grupo de edad, IMC (272),

Tabla 4b. Protocolo de entrenamiento de fuerza.

\begin{tabular}{|c|c|c|c|}
\hline Semana & $\%$ 1RM & $\mathbf{N}^{\circ}$ repetic. & $\mathbf{N}^{\circ}$ series \\
\hline $\mathbf{1}$ & 50 & 10 & 2 \\
\hline $\mathbf{2}$ & 60 & 10 & 2 \\
\hline $\mathbf{3}$ & 70 & 10 & 2 \\
\hline $\mathbf{4}$ & 70 & 10 & 3 \\
\hline $\mathbf{5}$ & 75 & 10 & 3 \\
\hline $\mathbf{6}$ & 80 & 10 & 3 \\
\hline $\mathbf{7}$ & 80 & 12 & 3 \\
\hline $\mathbf{8}$ & 85 & 12 & 3 \\
\hline
\end{tabular}

Esta tabla muestra el protocolo de progresión en las cargas (\% 1RM), así como en el número de repeticiones y series de cada ejercicio efectuado en el entrenamiento de fuerza de los pacientes analizados. 


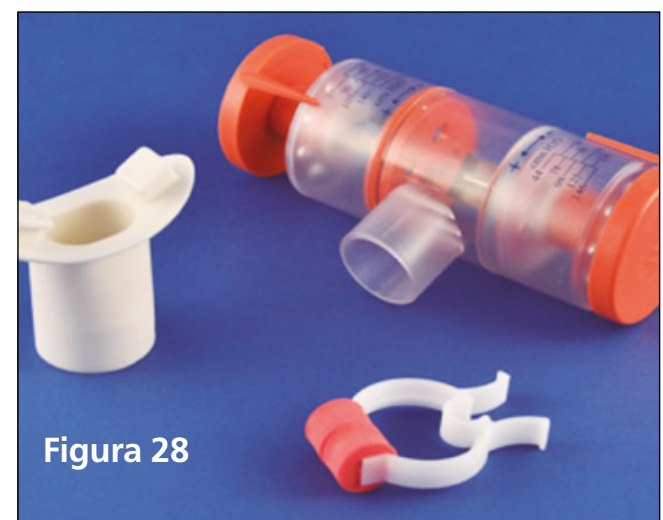

Figura 28. Válvula de entrenamiento de músculos respiratorios de tipo umbral, Orygendual $\circledast$. hábito tabáquico, uso de $O C D$, nivel de actividad física habitual (2), número de exacerbaciones (45), variación en la función pulmonar durante el período de seguimiento, incremento en la distancia alcanzada en el 6MWT (clínicamente importante o no) $(249,273-276)$ y período de seguimiento. Para la comparación intergrupo en todos los niveles de segmentación se utilizaron pruebas paramétricas ( $T$ para 2 muestras, ANOVA y prueba de Welch) y no paramétricas (U de Mann-Whitney y Kruskal-Wallis), según la distribución de la muestra. Se evaluaron las condiciones de aplicabilidad para un análisis de covarianza, mediante la evaluación de la normalidad de los residuos del modelo de regresión (utilizando la prueba de Shapiro-Wilk), pero no se cumplían para ninguna de los variables independientes.

Por último, se analizó la influencia de distintas variables (edad, IMC, FEV $(\%)$, número de exacerbaciones e incremento en la distancia del 6MWT post-tratamiento) sobre el deterioro en la tolerancia al ejercicio tras el período de seguimiento, a través de los coeficientes de correlación de Pearson y Spearman (según distribución de la muestra), controlando por factores de confusión. Así mismo, se aplicó un modelo de regresión lineal para determinar el impacto de la asociación entre las variables con una correlación significativa.

Para el análisis de normalidad de todas las variables incluidas en el estudio se utilizó la prueba de Shapiro-Wilk. En todos los análisis se asumió un nivel de confianza $(1-\alpha)$ de 0,95 (nivel de significación $\alpha=0,05$ ). Así mismo, se especifica cuando se producen resultados muy significativos (nivel de significación $\alpha=0,01$ ).

\section{Consideraciones éticas}

Se trata de un estudio observacional, en el que no se llevó a cabo ninguna intervención adicional sobre los sujetos de la muestra, sino que sólo se ha recopilado y analizado información

Tabla 4c. Protocolo de entrenamiento específico de músculos respiratorios.

\begin{tabular}{|c|c|c|c|c|c|c|}
\hline \multirow{2}{*}{ Semana } & \multicolumn{2}{|c|}{ \% PIM-PEM } & \multicolumn{2}{|c|}{$\mathrm{N}^{\circ}$ repeticiones por ciclo } & \multirow{2}{*}{$\mathrm{N}^{\circ} \operatorname{ciclos}^{(1)}$} & \multirow{2}{*}{$\begin{array}{c}\text { Reposo } \\
\text { (min) }\end{array}$} \\
\hline & Ent. Resistencia & Ent. Fuerza & Ent. Resistencia & Ent. Fuerza & & \\
\hline 1 & 30 & - & 10 & 0 & 15 & 1 \\
\hline 2 & 40 & - & 10 & 0 & 15 & 1 \\
\hline 3 & 50 & 70 & 7 & 3 & 15 & 1 \\
\hline 4 & 50 & 80 & 7 & 3 & 15 & 1 \\
\hline 5 & 60 & 90 & 7 & 3 & 15 & 1 \\
\hline 6 & 60 & 90 & 7 & 3 & 15 & 1 \\
\hline 7 & 70 & 100 & 7 & 3 & 15 & 1 \\
\hline 8 & 70 & 100 & 7 & 3 & 15 & 1 \\
\hline
\end{tabular}

Esta tabla muestra el protocolo de progresión en las cargas según el porcentaje de las presiones inspiratoria y espiratoria máximas evaluadas al inicio del programa (\% PIM-PEM), así como en el número de repeticiones que constituye cada ciclo de trabajo, el número de ciclos completos y el tiempo de reposo entre ciclos.

(1) Número de ciclos en cada período de entrenamiento (dos períodos al día) 
sobre unas actuaciones terapéuticas ya ejecutadas. Además, el tratamiento aplicado en los tres grupos no tuvo implicaciones nocivas o riesgos en ningún caso, más bien al contrario.

En cuanto al tratamiento de los datos personales y clínicos, se ha seguido lo establecido en la Ley 15/1999 de Protección de Datos de Carácter General y en la Ley 41/2000, reguladora de la autonomía del paciente y de derechos y obligaciones en materia de información y documentación clínica, garantizando en todo momento el derecho a la intimidad y a la privacidad de los pacientes. Además, se han utilizado códigos numéricos asignados a cada sujeto, empleando éstos para su identificación en lugar de sus datos personales.

Cabe destacar que a todos los pacientes tratados en la Escuela Universitaria de Fisioterapia de la ONCE se les aportaba una hoja informativa acerca de la terapia que iban a recibir (características, objetivos, beneficios y riesgos), y previo al inicio de las sesiones, debían prestar su conformidad mediante la firma del consentimiento informado (en el Anexo II se muestra la hoja de información específica para pacientes que reciben un PRR y el formulario de consentimiento Informado del Centro). En este documento, además de prestar su conformidad para someterse al tratamiento en cuestión, también aceptan que sus datos sean utilizados con fines docentes o de investigación. No obstante a esto, a los pacientes seleccionados para formar parte de la muestra de estudio, se les informó del proyecto y se les pidió nuevamente la conformidad para la utilización de sus datos (Anexo III). 


\section{RESULTADOS}

\section{Características de la población de estudio}

Se ha analizado a un total de 35 pacientes diagnosticados de EPOC ( 28 hombres y 7 mujeres), con una edad comprendida entre 41 y 79 años, de los cuales, 8 recibieron el PRR 1, 16 el PRR 2 y 11 el PRR 3. En la Tabla 5 se detallan las características generales de la muestra completa y segmentada según el tipo de entrenamiento, al inicio del estudio.

\section{Descripción de los resultados obtenidos en la muestra completa y segmentada según el tipo de entrenamiento}

\subsection{Resultados en la muestra completa (Tabla 6a)}

\section{Post-tratamiento}

Tras el entrenamiento, se produjo un incremento en la distancia caminada en el 6MWT $(59,1 \pm 27,3 \mathrm{~m} ; 95 \% \mathrm{IC}$. $(49,7 ; 68,5) ; \mathrm{p}=0,000)$. Esta mejoría supuso un aumento de un $14,5 \%$ en relación a los registros previos al entrenamiento. En cuanto al resto de variables recogidas

Tabla 5. Características basales de la muestra completa y segmentada según el programa de entrenamiento aplicado.

\begin{tabular}{|c|c|c|c|c|c|c|c|c|}
\hline & \multicolumn{2}{|c|}{$\begin{array}{l}\text { Total } \\
n=35\end{array}$} & \multicolumn{2}{|c|}{$\begin{array}{l}\text { Ent. Resistencia } \\
\qquad n=8\end{array}$} & \multicolumn{2}{|c|}{$\begin{array}{c}\text { Ent. } \\
\text { Resistencia-Fuerza } \\
n=16\end{array}$} & \multicolumn{2}{|c|}{$\begin{array}{l}\text { Ent. Resistencia-Fuerza- } \\
\text { Músculos Respiratorios } \\
\qquad n=11\end{array}$} \\
\hline Sexo & \multicolumn{2}{|c|}{27 hombres (77\%) } & \multicolumn{2}{|c|}{6 hombres $(75 \%)$} & \multicolumn{2}{|c|}{13 hombres (81\%) } & \multicolumn{2}{|c|}{8 hombres $(73 \%)$} \\
\hline $\begin{array}{l}\text { Clasificación } \\
\text { EPOC (GOLD) }\end{array}$ & \multicolumn{2}{|c|}{$\begin{array}{c}5 \text { Grado I }(13,5 \%) \\
8 \text { Grado II }(21,6 \%) \\
4 \text { Grado III }(10,8 \%) \\
18 \text { Grado IV }(48,6 \%)\end{array}$} & \multicolumn{2}{|c|}{$\begin{array}{l}2 \text { Grado I }(25 \%) \\
3 \text { Grado II }(37,5 \%) \\
1 \text { Grado III }(12,5 \%) \\
2 \text { Grado IV }(2,5 \%)\end{array}$} & \multicolumn{2}{|c|}{$\begin{array}{c}1 \text { Grado I }(6,3 \%) \\
3 \text { Grado II }(18,8 \%) \\
0 \text { Grado III }(0 \%) \\
12 \text { Grado IV }(75 \%)\end{array}$} & \multicolumn{2}{|c|}{$\begin{array}{l}2 \text { Grado I }(18,2 \%) \\
2 \text { Grado II }(18,2 \%) \\
3 \text { Grado III }(27,3 \%) \\
4 \text { Grado IV }(36,4 \%)\end{array}$} \\
\hline $\begin{array}{l}\text { Hábito } \\
\text { tabáquico }\end{array}$ & \multicolumn{2}{|c|}{31 exfumadores $(88,8 \%)$} & \multicolumn{2}{|c|}{7 exfumadores $(87,5 \%)$} & \multicolumn{2}{|c|}{14 exfumadores $(87,5 \%)$} & \multicolumn{2}{|c|}{10 exfumadores $(90,9 \%)$} \\
\hline \multirow[t]{2}{*}{ Uso de OCD } & \multicolumn{2}{|c|}{5 con OCD $(14,3 \%)$} & \multicolumn{2}{|c|}{0 con $0 C D(0 \%)$} & \multicolumn{2}{|c|}{4 con OCD $(25 \%)$} & \multicolumn{2}{|c|}{1 con OCD $(9,1 \%)$} \\
\hline & Media $\pm D E$ & Mín-Max & Media $\pm D E$ & Mín-Max & Media $\pm D E$ & Mín-Max & Media $\pm D E$ & Mín-Max \\
\hline Edad & $61,4 \pm 11,2$ & $41-79$ & $54,5 \pm 9,8$ & $41-69$ & $64,5 \pm 11,2$ & $43-78$ & $62 \pm 10,9$ & $45-79$ \\
\hline $\begin{array}{l}\text { Evol. } \\
\text { enfermedad } \\
\text { (años) }\end{array}$ & $6,1 \pm 4,9$ & $0-18$ & $4,1 \pm 3,3$ & $0-8$ & $7,8 \pm 5,2$ & $1-18$ & $5,2 \pm 5,3$ & $1-17$ \\
\hline $\begin{array}{l}\text { Período sin } \\
\text { fumar (años) }\end{array}$ & $6,7 \pm 4,9$ & $1-18$ & $4,1 \pm 2,9$ & $1-8$ & $8,3 \pm 4,5$ & $1-15$ & $6,2 \pm 6,1$ & $1-18$ \\
\hline Paq/año & $44,1 \pm 15,7$ & $18-77$ & $38,5 \pm 16,2$ & $18-62$ & $45,8 \pm 17,8$ & $20,5-77$ & $45,5 \pm 12,4$ & $20,5-61$ \\
\hline IMC $\left(\mathrm{Kg} / \mathrm{m}^{2}\right)$ & $26,7 \pm 4,3$ & $21,3-38,3$ & $29,3 \pm 4,9$ & $23,5-38,3$ & $25,6 \pm 3,8$ & $21,4-33,3$ & $26,4 \pm 4,2$ & $21,3-35,5$ \\
\hline $\mathrm{FEV}_{1}(\mathrm{~L})$ & $1,21 \pm 0,48$ & $0,68-2,44$ & $1,54 \pm 0,56$ & $0,95-2,22$ & $1,02 \pm 0,42$ & $0,68-2,44$ & $1,23 \pm 0,42$ & $0,71-1,97$ \\
\hline $\mathrm{FEV}_{1}(\%)$ & $41 \pm 19$ & $18,5-77,5$ & $51,7 \pm 17,6$ & $28,2-75,8$ & $34,3 \pm 17,7$ & $18,6-70,1$ & $42,9 \pm 19,5$ & $18,5-77,5$ \\
\hline FVC (L) & $2,52 \pm 0,68$ & $0,9-3,69$ & $2,95 \pm 0,73$ & $1,85-3,67$ & $2,24 \pm 0,63$ & $0,9-3,51$ & $2,53 \pm 0,52$ & $1,71-3,22$ \\
\hline FVC $(\%)$ & $50,8 \pm 11,6$ & $31,9-71,8$ & $58,4 \pm 8,7$ & $46,6-70,8$ & $43,1 \pm 11,4$ & $31,9-70,1$ & $52,4 \pm 11,3$ & $37,7-70,6$ \\
\hline $\mathrm{FEV}_{1} / \mathrm{FVC}(\%)$ & $46,1 \pm 10,9$ & $27,4-78,5$ & $51,4 \pm 8,2$ & $38,8-60,2$ & $43,1 \pm 10,3$ & $27,4-69,5$ & $46,5 \pm 12,5$ & $29,7-78,5$ \\
\hline Índice BODE & $3,1 \pm 1,5$ & $0-6$ & $2,2 \pm 1,4$ & $0-4$ & $3,3 \pm 1,1$ & $1-5$ & $3,5 \pm 1,8$ & $0-6$ \\
\hline
\end{tabular}


Pie tabla 5. Los datos se presentan para la muestra completa y cada grupo de entrenamiento: entrenamiento de resistencia aislado (Ent. Resistencia); entrenamiento de resistencia y fuerza de miembros superiores e inferiores (Ent. Resistencia-Fuerza); entrenamiento de resistencia y fuerza de los miembros más entrenamiento específico de los músculos respiratorios (Ent. Resistencia-Fuerza-Músculos Respiratorios).

Las variables cualitativas sexo, clasificación de la EPOC según los criterios de la Iniciativa GOLD (Global Obstructive Lung Disease) de 2001, hábito tabáquico (exfumadores / no fumadores) y la utilización o no de oxigenoterapia continua domiciliaria (OCD), se expresan con sus frecuencias absolutas y relativas (\%).

Para las variables cuantitativas, los datos se exponen como media y desviación estándar (DE) y con el rango (Mín-Máx): Evol. de la enfermedad: años de evolución de la enfermedad desde su diagnóstico; Período sin fumar: años transcurridos desde el cese del hábito tabáquico en los sujetos exfumadores; Paq/año: valor de paquetes/año para los sujetos exfumadores; IMC: índice de masa corporal; FEV en el primer segundo; FVC: capacidad vital forzada; FEV (\%) y FVC (\%)): porcentaje del FEV y de la FVC en relación al valor teórico.

Se verifica la homogeneidad intergrupo en todas las variables, evaluada mediante (a) Prueba Exacta de Fisher, (b) ANOVA y (c) Kruskal-Wallis ( $p>0,05)$.

en la prueba, se obtuvo una reducción significativa en la disnea, la fatiga muscular y en la desaturación, mientras que no se observaron cambios significativos en la frecuencia cardiaca (ver detalle en Tabla 6a).

En relación a la disnea basal, se redujo la puntuación de la Escala mMRC en un 36,8\% respecto a los datos pre-intervención $(-0,7 \pm 0,4 ; 95 \%$ IC. $(-0,9 ;-0,5) ; p=0,000)$.

\section{Post-seguimiento}

El seguimiento osciló entre 9 y 18 meses (media de 12,7士2,9). Durante este período no se recogieron incidencias relevantes y la función pulmonar en los sujetos se mantuvo estable (como muestra la Tabla 6a, no se encontraron diferencias significativas en la variación del FEV ${ }_{1} \mathrm{FEV}_{1}$ (\%), FVC, FVC (\%) y FEV/FVC).

Del mismo modo, el número de exacerbaciones fue similar al que presentaron en los 12 meses anteriores (media de exacerbaciones previa de 0,6 (rango, 0-4) frente a 0,7 (rango, 0-6) de exacerbaciones en el período de seguimiento, normalizado según su duración.

En cuanto al $6 \mathrm{MWT}$, se produjo un descenso de un $10,6 \%$ en la distancia alcanzada en la prueba en comparación con la medición post-entrenamiento $(-49,8 \pm 23$ m; $95 \%$ IC. $(-57,6 ;-40)$; $\mathrm{p}=0,000$ ). Igualmente, se incrementaron de forma significativa la disnea y fatiga percibidas durante la prueba, así como la desaturación, mientras que no se produjeron cambios relevantes en la frecuencia cardiaca (ver detalle en Tabla 6a).

Por otro lado, se produjo un incremento del $36,8 \%$ en la puntuación de la Escala mMRC $(0,7 \pm 0,4 ; 95 \%$ IC. $(0,6 ; 0,8) ; p=0,000)$.

Por último, el rango de hora semanales de ejercicio físico fue de 0-6 en el registro pre-intervención y de 0-7 en el registro post-seguimiento. No se observaron cambios en el nivel de actividad física de los sujetos $(0 \pm 1,4$ horas; $95 \% I C$. $(-0,4 ; 0,4) ; p=1,000)$.

En las Gráficas 4, 5 y 6 se representa la evolución de estas variables a lo largo del estudio, para la muestra completa y segmentada segúnel tipode entrenamiento.

\subsection{Resultados comparativos según el tipo de entrenamiento (Tabla 6b) ${ }^{1}$}

\section{Post-entrenamiento}

El grupo con mayor incremento en el número de metros alcanzados en la prueba de marcha fue el sometido al PRR $2\left(66,3 \pm 23,6\right.$ m; $95 \%$ IC. $\left.(53,7 ; 78,9) ; p=0,000^{f}\right)$, que mejoró un 15,6\% en relación a los datos pre-intervención. El grupo con peores resultados fue el del PRR 1 $(46,1 \pm 40 \mathrm{~m} ; 95 \% \mathrm{IC}$. $(1,4 ; 79,8) ; \mathrm{p}=0,015 \mathrm{e})$, que aumentó la distancia en un $11,7 \%$. El grupo de 
Tabla 6a. Efectos de los programas de Rehabilitación Respiratoria a corto y largo plazo (muestra completa).

\begin{tabular}{|c|c|c|c|c|c|}
\hline \multirow[b]{2}{*}{$\mathrm{n}=35$} & \multirow[b]{2}{*}{ Pre-Intervención } & \multirow[b]{2}{*}{ Post-Intervención } & \multirow[b]{2}{*}{ Post-Seguimiento } & \multicolumn{2}{|c|}{ Intragrupo p } \\
\hline & & & & $\begin{array}{l}\text { Post }_{\text {Int }} \\
\text { vs } \\
\text { Pre }_{\text {Int }}\end{array}$ & $\begin{array}{l}\text { Post }_{\text {seg }} \\
\text { vs } \\
\text { Post| }\end{array}$ \\
\hline Distancia 6MWT (m) & $408,5 \pm 64,9$ & $467,5 \pm 74,8$ & $417,5 \pm 69,6$ & $0,000^{(f)}$ & $0,000^{(f)}$ \\
\hline$\Delta$ Disnea - 6MWT (Borg) & $3,9 \pm 1,5$ & $3,2 \pm 1,3$ & $3,9 \pm 1,2$ & $0,013^{(f)}$ & $0,027^{(f)}$ \\
\hline$\Delta$ Fatiga - 6MWT (Borg) & $1,5 \pm 0,9$ & $0,7 \pm 0,7$ & $1,5 \pm 0,9$ & $0,000^{(f)}$ & $0,000^{(f)}$ \\
\hline$\Delta \mathrm{FC}-6 \mathrm{MWT}(\mathrm{lpm})$ & $32,2 \pm 14$ & $34,3 \pm 10,6$ & $33,3 \pm 15,3$ & $0,152^{(\mathrm{e})}$ & $0,649^{(e)}$ \\
\hline$\Delta$ SatO $_{2}-6 M W T(\%)$ & $-7,6 \pm 5,8$ & $-6 \pm 4,7$ & $-7,9 \pm 5,5$ & $0,009^{(f)}$ & $0,002^{(f)}$ \\
\hline Disnea basal (mMRC) & $1,9 \pm 0,7$ & $1,2 \pm 0,4$ & $1,9 \pm 0,7$ & $0,000^{(f)}$ & $0,000^{(f)}$ \\
\hline $\mathrm{FEV}_{1}(\mathrm{~L})$ & $1,31 \pm 0,58$ & - & $1,19 \pm 0,51$ & - & $0,840^{(f)}$ \\
\hline $\mathrm{FEV}_{1}(\%)$ & $41 \pm 19$ & - & $40,3 \pm 19,5$ & - & $0,193^{(f)}$ \\
\hline FVC (L) & $2,52 \pm 0,68$ & - & $2,49 \pm 0,61$ & - & $0,580^{(f)}$ \\
\hline FVC (\%) & $50,8 \pm 11,6$ & - & $42,1 \pm 30,8$ & - & $0,129^{(f)}$ \\
\hline $\mathrm{FEV}_{1} / \mathrm{FVC}(\%)$ & $46,1 \pm 10,8$ & - & $45,9 \pm 10,7$ & - & $0,801^{(f)}$ \\
\hline $\mathrm{N}^{\circ}$ exacerbaciones & $0,6 \pm 0,5$ & - & $0,7 \pm 1$ & - & $0,721^{(f)}$ \\
\hline Horas semanales de ejercicio & $1,6 \pm 2,1$ & - & $1,6 \pm 2,2$ & - & $1,000^{(f)}$ \\
\hline
\end{tabular}

Los datos se presentan como media y desviación estándar para los tres momentos de medición: previa al inicio de los programas, al finalizar éstos y tras un período de seguimiento sin mantenimiento.

Distancia 6MWT (m): distancia alcanzada en la Prueba de Marcha de Seis Minutos; $\Delta$ Disnea - 6 MWT (Borg), $\Delta$ Fatiga - 6MWT (Borg), $\Delta \mathrm{FC}$ - 6MWT (lpm) y $\Delta \mathrm{SatO}_{2}-6 \mathrm{MWT}$ (\%): variación en las variables recogidas durante el desarrollo de la Prueba de Marcha de Seis Minuto (disnea y fatiga según Escala de Borg modificada, frecuencia cardiaca y saturación de oxígeno, respectivamente); Disnea basal (mMRC): disnea basal según Escala Modificada del Medical Research Council; FEV : volumen espiratorio forzado en el primer segundo; FVC: capacidad vital forzada; $\mathrm{FEV}_{1}(\%)$ y FVC (\%)): porcentaje del FEV y de la FVC en relación al valor teórico; $\mathrm{N}^{\circ}$ exacerbaciones: número de exacerbaciones sufridas en los 12 meses anteriores (medición Pre-Intervención) o durante el período de seguimiento (medición Post-Seguimiento).

Los valores de $p$ para las comparaciones intragrupo de las variables han sido obtenidos mediante: (e) T para datos pareados y (f) Wilcoxon.

PRR 3 también obtuvo resultados positivos $\left(57,9 \pm 20 \mathrm{~m} ; 95 \% \mathrm{IC} .(55,2 ; 61,1) ; \mathrm{p}=0,003^{\mathrm{e}}\right)$, con un incremento porcentual del $14,7 \%$. Sin embargo, estas diferencias entre los grupos no fueron estadísticamente significativas. En cuanto al resto de variables recogidas durante el desarrollo del 6MWT, sólo se encontraron diferencias significativas entre los grupos en la variación en la saturación de oxígeno (ver detalle de ésta y el resto de variables en la Tabla 6b).

En relación a la disnea basal, el grupo que más redujo la puntuación en la Escala mMRC fue el que recibió el PRR $3\left(-0,8 \pm 0,4 ; 95 \%\right.$ IC. $\left.(-1,1 ;-0,5) ; p=0,003^{f}\right)$, seguido del grupo de PRR $2\left(-0,7 \pm 0,5 ; 95 \%\right.$ IC. $\left.(-1 ;-0,4) ; p=0,001^{\dagger}\right)$ y el que menos, el que recibió el PRR 1 $\left(-0,6 \pm 0,5 ; 95 \%\right.$ IC. $\left.(-1,1 ;-0,1) ; p=0,025^{f}\right)$. Sin embargo, realizando un análisis porcentual del cambio en relación a los datos pre-intervención, el grupo de PRR 2 redujo su disnea en un $36,8 \%$, seguido muy de cerca del grupo de PRR 3, con una reducción del $36,4 \%$ y por último, el grupo de PRR 1, con un 33,3\%. Estas diferencias entre los grupos no fueron estadísticamente significativas.

Por otro lado, en el grupo en que se evaluaron las presiones respiratorias (PRR 3), se observó un incremento significativo de la PIM $\left(11,7 \pm 2,7 \mathrm{cmH}_{2} \mathrm{O} ; 95 \% \mathrm{IC} .(9,8 ; 13,6) ; \mathrm{p}=0,000^{\circ}\right)$ y de la 
PEM $\left(16,4 \pm 1,9 \mathrm{cmH} 20 ; 95 \% \mathrm{IC} .(14,6 ; 18,2) ; p=0,000^{\circ}\right)$, lo que representaba una mejoría de un $19 \%$ y de un $21 \%$, respectivamente, en relación a los datos pre-intervención.

\section{Post-seguimiento}

Tras el período de seguimiento, los pacientes que recibieron el PRR 3 fueron los que presentaron una menor pérdida en la distancia alcanzada en el 6MWT tanto en valor absoluto $\left(-43,3 \pm 10,4 \mathrm{~m} ; 95 \% \mathrm{IC}\right.$. $\left.(-53,2 ;-33,6) ; p=0,003^{f}\right)$ como en valor porcentual (reducción

Gráfica 4. Efecto del entrenamiento sobre la tolerancia al ejercicio, evaluada mediante la Prueba de Marcha de Seis Minutos, para la muestra completa y segmentada según tipo de entrenamiento.

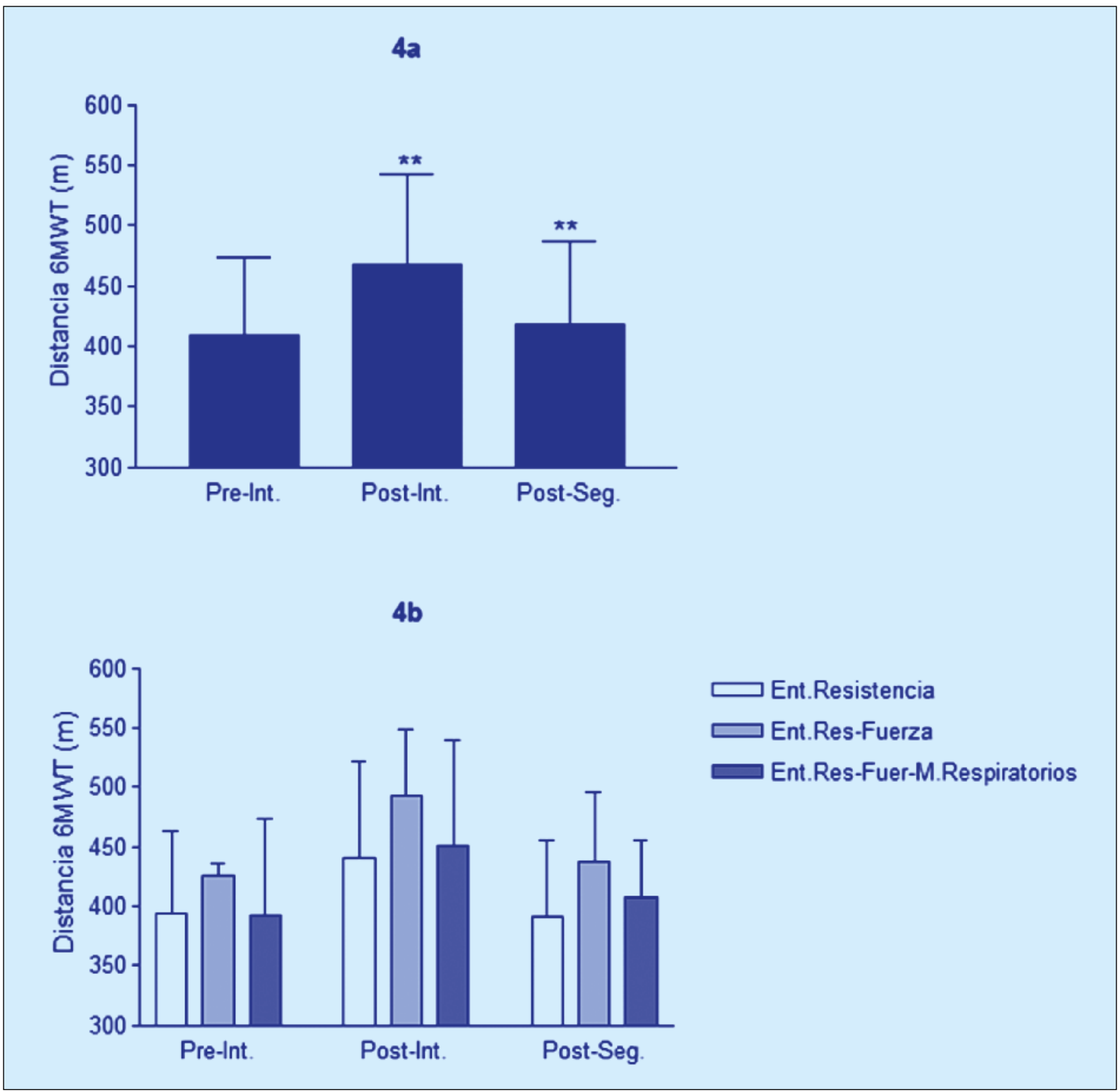

Se representa la media y la desviación estándar de la distancia alcanzada en la Prueba de Marcha de Seis Minutos (6MWT) en los distintos momentos de medición analizados: Previo al comienzo de los programas (Pre-Int.), a la finalización de los mismos (Post-Int.) y tras un período de seguimiento sin mantenimiento (Post-Seg.).

4 a representa los resultados para la muestra completa, siendo. $(* *), p<0,01$ para la comparación postintervención vs pre-intervención y post-seguimiento vs post-intervención.

4b representa los resultados para los distintos grupos de entrenamiento: Entrenamiento de resistencia (Ent.Resistencia), entrenamiento de resistencia y fuerza de miembros (Ent.Res-Fuerza) y entrenamiento de resistencia-fuerza y específico de músculos respiratorios (Ent.Res-Fuer-M.Respiratorios). No se hallaron diferencias significativas entre los grupos para la comparación post-intervención vs pre-intervención ni postseguimiento vs post-intervención. 
del 9,7\% en relación a los resultados post-entrenamiento). El grupo que recibió el PRR 1 fue en el que se observó una pérdida mayor $(-50 \pm 28 \mathrm{~m} ; 95 \% \mathrm{IC}$. $(-73,7 ;-26,3) ; p=0,001 \mathrm{e})$, que constituyó una reducción del 11,3\%. Por su parte, los sujetos incluidos en el PRR 2 también perdieron distancia caminada $(-54,7 \pm 26,1 \mathrm{~m}$; 95\% IC. $(-69 ;-40,4) ; p=0,000)$, un $11,1 \%$ de decremento porcentual. Estas diferencias entre grupos en el período de seguimiento, no fueron estadísticamente significativas. En cuanto al resto de variables recogidas durante

Gráfica 5. Efecto del entrenamiento sobre la disnea basal para la muestra completa y segmentada según el tipo de entrenamiento.

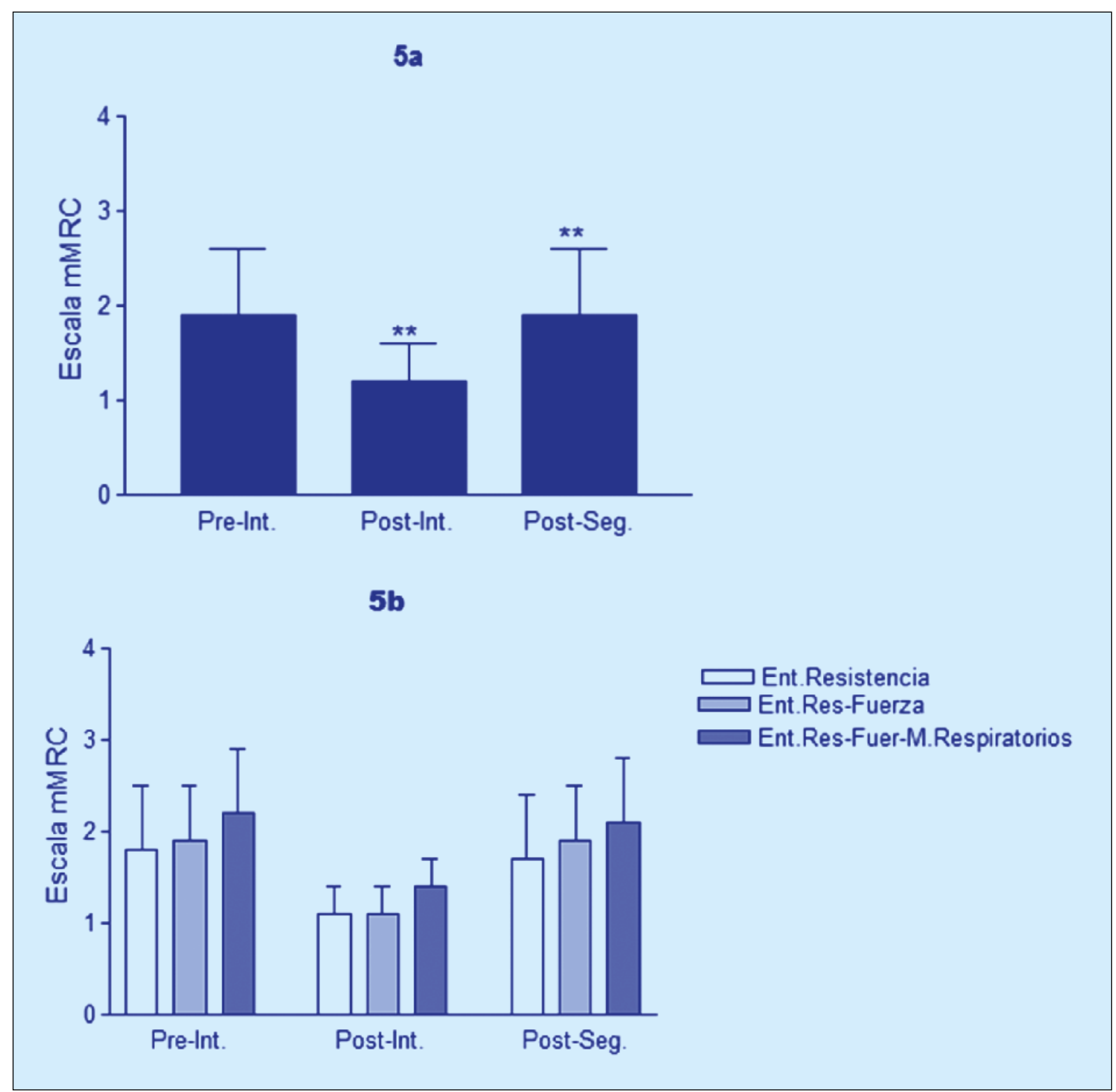

Se representa la media y la desviación estándar de la puntuación obtenida en la Escala Modificada del Medical Research Council (mMRC) en los distintos momentos de medición analizados: Previo al comienzo de los programas (Pe-Int.), a la finalización de los mismos (Post-Int.) y tras un período de seguimiento sin mantenimiento (Post-Seg.).

5 a representa los resultados para la muestra completa, siendo. $(* *), p<0,01$ para la comparación postintervención vs pre-intervención y post-seguimiento vs post-intervención.

$5 \mathrm{~b}$ representa los resultados para los distintos grupos de entrenamiento: Entrenamiento de resistencia (Ent.Resistencia), entrenamiento de resistencia y fuerza de miembros (Ent.Res-Fuerza) y entrenamiento de resistencia-fuerza y específico de músculos respiratorios (Ent.Res-Fuer-M.Respiratorios). No se hallaron diferencias significativas entre los grupos para la comparación post-intervención vs pre-intervención ni postseguimiento vs post-intervención. 
el desarrollo del 6MWT, sólo se encontraron diferencias significativas entre los grupos en la variación en la saturación de oxígeno (ver detalle de ésta y el resto de variables en la Tabla 6b).

En relación a la disnea basal, ésta se incrementó en todos los grupos prácticamente en la misma medida en que se redujo tras el entrenamiento. El grupo de PRR 1 en un 35,3\% $\left(0,6 \pm 0,5 ; 95 \%\right.$ IC. $\left.(0,2 ; 1) ; p=0,025^{f}\right)$, en el grupo de PRR 2 en un $36,8 \%(0,7 \pm 0,5 ; 95 \%$ IC. $(0,2 ;$ $\left.1,2) ; p=0,001^{f}\right)$ y en el grupo de PRR 3 en un $36,4 \%\left(0,8 \pm 0,4 ; 95 \% I C .(0,5 ; 1,1) ; p=0,003^{f}\right)$. No se hallaron diferencias significativas entre los grupos para esta variable.

Gráfica 6. Efecto del entrenamiento sobre el nivel de actividad física habitual para la muestra completa y segmentada según el tipo de entrenamiento.

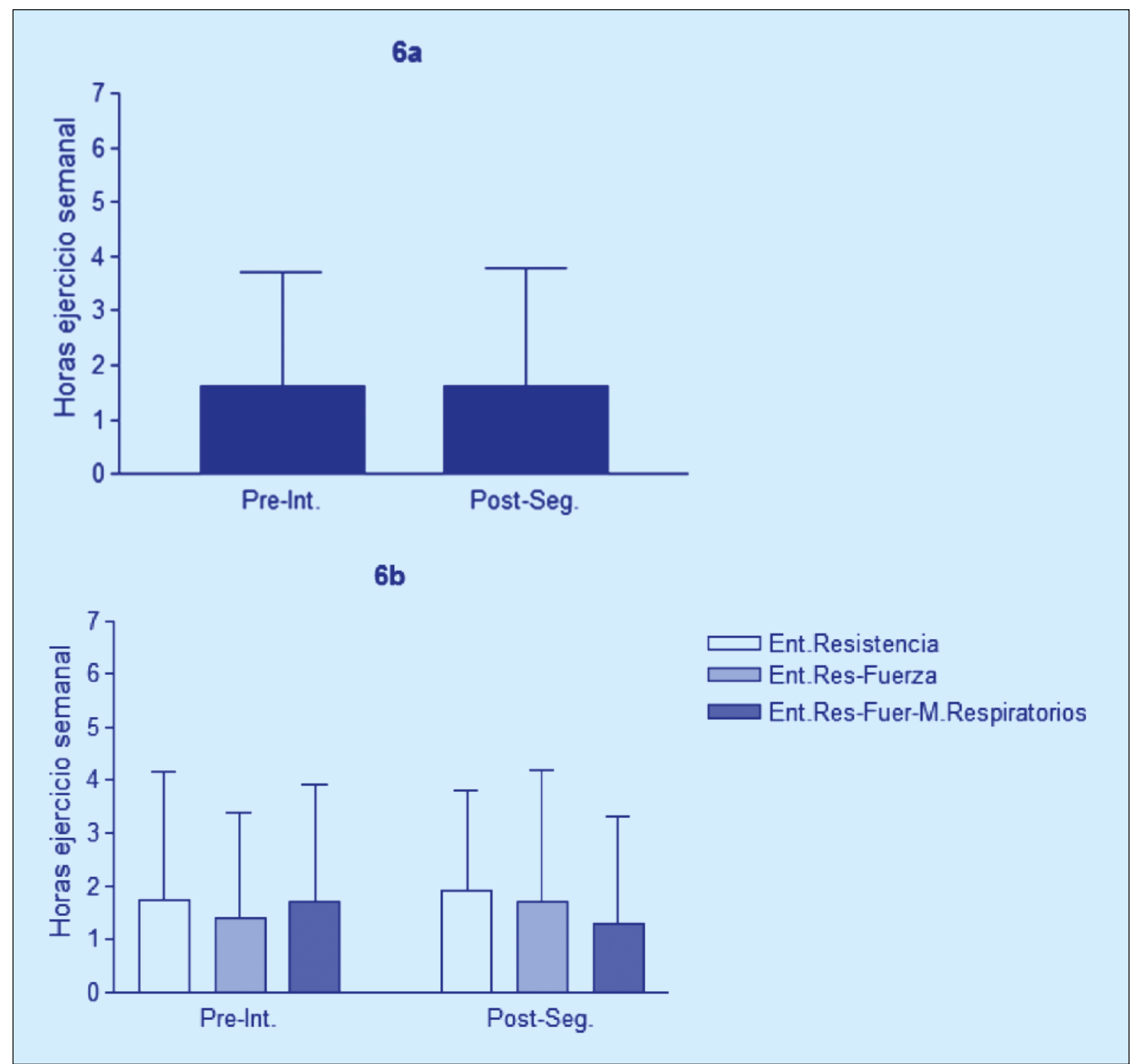

Se representa la media y la desviación estándar del número de horas de ejercicio físico semanal que realizaban los sujetos incluidos en el estudio, antes de comenzar los programas (Pre-Int.) y tras el período de seguimiento (Post-Seg.).

6 a representa los resultados para la muestra completa. No se encontraron diferencias significativas postseguimiento vs pre-intervención.

$6 \mathrm{~b}$ representa los resultados para los distintos grupos de entrenamiento: Entrenamiento de resistencia (Ent.Resistencia), entrenamiento de resistencia y fuerza de miembros (Ent.Res-Fuerza) y entrenamiento de resistencia-fuerza y específico de músculos respiratorios (Ent.Res-Fuer-M.Respiratorios). No se hallaron diferencias significativas entre los grupos para la comparación post-seguimiento vs pre-intervención. 


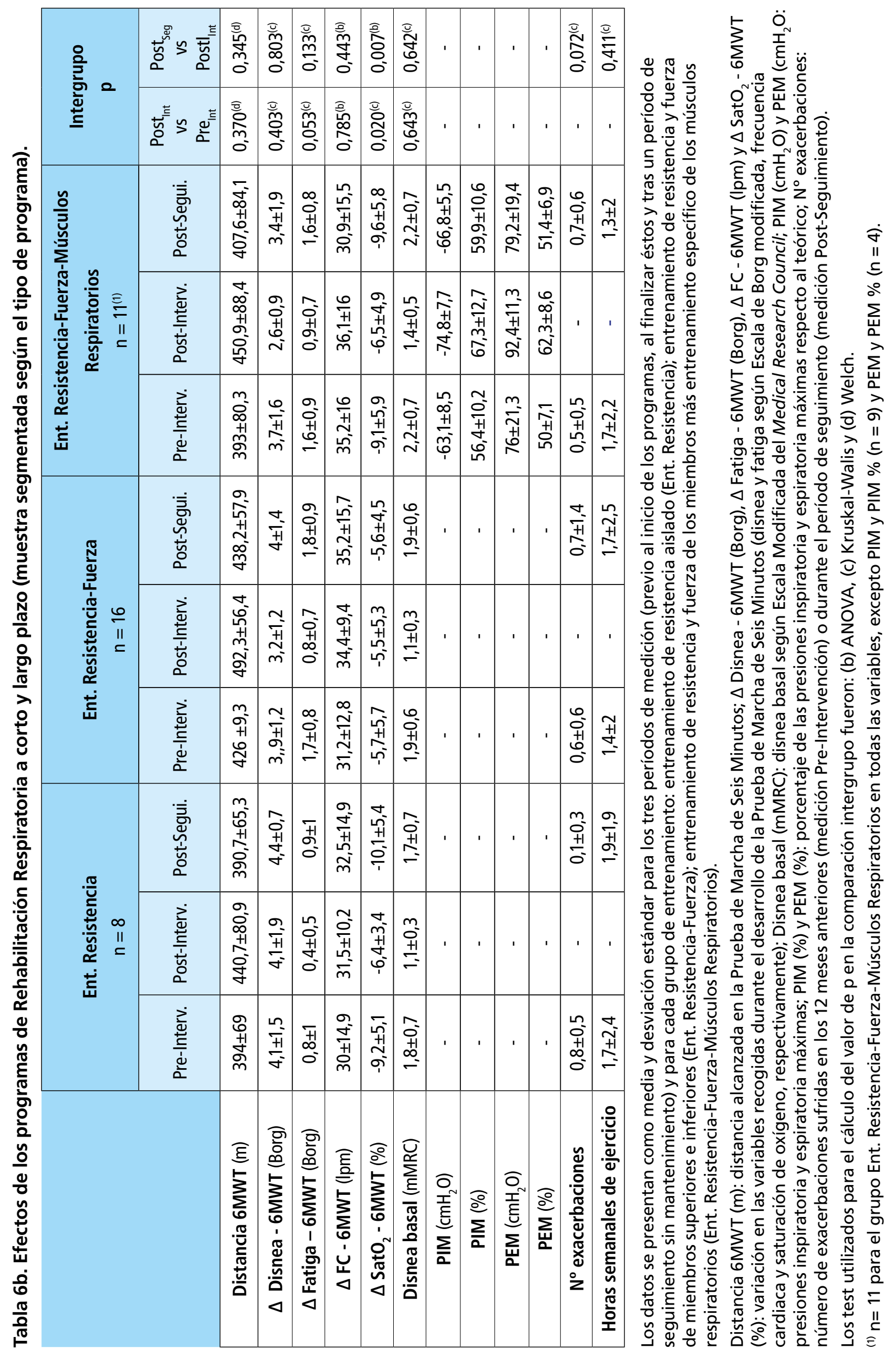


Por otro lado, tras el período de seguimiento, se observó un descenso significativo de la PIM $\left(-8 \pm 2 \mathrm{cmH}_{2} \mathrm{O} ; 95 \%\right.$ IC. $\left.(-9,3 ;-6,7) ; p=0,00^{\circ}\right)$ y de la PEM $\left(-13,2 \pm 1,6 \mathrm{cmH}_{2} \mathrm{O} ; 95 \%\right.$ IC. $(-16,5 ;-9,9) ; p=0,007)$, lo que representaba un empeoramiento de un $12 \%$ y de un $16,7 \%$, respectivamente, en relación a los datos post-intervención. En la Gráfica 7 se representan los valores de PIM y PEM durante los tres momentos de registro, para el grupo que entrenó músculos respiratorios.

Por último, indicar que en ningún grupo se produjo una modificación relevante ni en el número de horas de ejercicio físico semanal ni en las exacerbaciones, en comparación con los datos previos a la participación en los programas.

\section{Análisis de subgrupos ${ }^{1}$}

Se evaluó la existencia de diferencias significativas en los resultados de la variable principal (tolerancia al ejercicio), según distintos niveles de segmentación de la muestra que se detallan en la Tabla 7.

Sólo se hallaron diferencias significativas en la variación en la distancia alcanzada en el 6MWT según dos niveles de agrupamiento: número de exacerbaciones en los 12 meses previos a la realización del PRR e incremento en la distancia del 6MWT tras el entrenamiento. En la Tabla 8a se presentan las características de la muestra y en la Tabla 8b, los resultados detallados de la distancia alcanzada en el 6MWT post-entrenamiento y post-seguimiento para cada grupo.

Gráfica 7. Efecto del entrenamiento específico de los músculos respiratorios sobre las presiones respiratorias máximas.

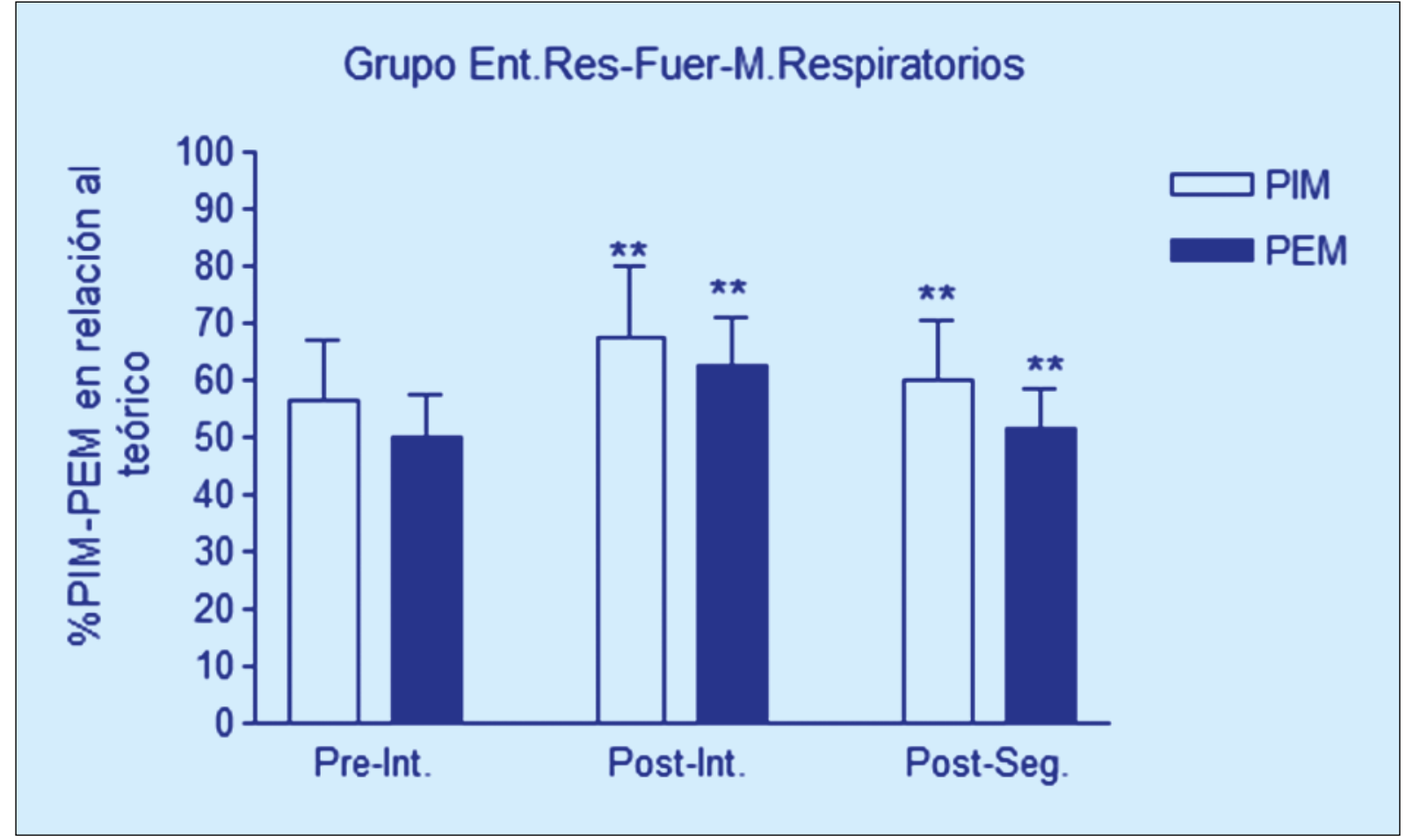

Se representa la media y la desviación estándar de las presiones inspiratoria y espiratoria máximas (porcentajes en relación al valor teórico obtenido según la ecuación de Morales (256)), para el grupo de tratamiento que recibió entrenamiento específico de músculos respiratorios, en los distintos momentos de medición analizados: Previo al comienzo de los programas (Pre-Int.), a la finalización de los mismos (Post-Int.) y tras un período de seguimiento sin mantenimiento (Post-Seg.).

(**, $p<0,01$ para la comparación post-intervención vs pre-intervención y post-seguimiento vs post-intervención. 
El grupo que no había sufrido exacerbaciones previas obtuvo un incremento del $17,3 \%$ en la distancia caminada en la prueba $\left(70,5 \pm 19,1 \mathrm{~m} ; 95 \% \mathrm{IC}\right.$. $\left.(60,9 ; 80,1) ; \mathrm{p}=0,000^{\mathrm{e}}\right)$. Por su parte, el grupo con 1-2 exacerbaciones también obtuvo una mejoría, aunque menor que el grupo anterior $(12,2 \%)$ y con una mayor dispersión de los resultados $(50 \pm 29,7 \mathrm{~m} ; 95 \% \mathrm{IC}$. $(37,4 ; 63,4)$; $\left.\mathrm{p}=0.000^{f}\right)$.

Tabla 7. Análisis de subgrupos: niveles de segmentación de la muestra.

\begin{tabular}{|c|c|}
\hline Grupos & Categorías de los grupos \\
\hline Sexo & $\begin{array}{l}\text { - Hombre } \\
\text { - Mujer }\end{array}$ \\
\hline Severidad de la EPOC según clasificación GOLD ${ }^{(1)}$ & $\begin{array}{l}\text { - Grados I-II } \\
\text { - Grados III-IV }\end{array}$ \\
\hline Grupo de edad & $\begin{array}{l}<50 \text { años } \\
50-65 \text { años } \\
>65 \text { años }\end{array}$ \\
\hline Índice de masa corporal(2) & $\begin{array}{l}\text { - Bajo peso }\left(<18,5 \mathrm{Kg} / \mathrm{m}^{2}\right) \\
\text { - Normopeso }\left(18,5-24,9 \mathrm{Kg} / \mathrm{m}^{2}\right) \\
\text { Sobrepeso }\left(\geq 25 \mathrm{Kg} / \mathrm{m}^{2}\right)\end{array}$ \\
\hline Hábito tabáquico & $\begin{array}{l}\text { Ex--fumador } \\
\text { - No fumador }\end{array}$ \\
\hline Uso de OCD & $\begin{array}{l}\text { - Sí } \\
\text { - No }\end{array}$ \\
\hline $\begin{array}{l}\text { Número de exacerbaciones en los } 12 \text { meses previos al inicio de los } \\
\text { programas y durante el seguimiento }{ }^{(3)}\end{array}$ & $\begin{array}{l}0 \text { exacerbaciones } \\
\text { 1-2 exacerbaciones } \\
-\geq 3 \text { exacerbaciones }\end{array}$ \\
\hline $\begin{array}{l}\text { Nivel de actividad física habitual previo al inicio de los programas y } \\
\text { durante el período de seguimiento( }{ }^{(4)}\end{array}$ & $\begin{array}{l}\text { - Bajo (<30 min/día) } \\
\text { - Moderado (30-120 min/día) } \\
\text { - Alto (>120 min/día) }\end{array}$ \\
\hline Período de seguimiento & $\begin{array}{l}\text { 9-12 meses } \\
>12 \text { meses }\end{array}$ \\
\hline Cambios en la función pulmonar durante el período de seguimiento & $\begin{array}{l}-\downarrow \mathrm{FEV}_{1}<10 \% \\
-\downarrow \mathrm{FEV}_{1} 10-30 \% \\
-\downarrow \mathrm{FEV}_{1}>30 \%\end{array}$ \\
\hline Incremento en la distancia del 6MWT tras el entrenamiento(5) & $\begin{array}{l}<30 \text { metros } \\
\geq 30 \text { metros }\end{array}$ \\
\hline
\end{tabular}

(1) Global Iniciatve Obstructive Lung Disease (43).

(2)Según la clasificación de la Organización Mundial de la Salud (272).

${ }^{(3)}$ Siguiendo la estratificación del índice BODEx (Body Mass Index, Obstruction, Dyspnea and Exacerbations) (45).

(4)Según los criterios descritos por la Guía GesEPOC de 2012 (2).

${ }^{(5)}$ Según distancia mínima clínicamente importante. Se establecen 30 metros como punto de corte para la categorización, a pesar de que no existe un consenso al respecto, encontrándose en la literatura valores de 25 m (274), 30 m (276) y 35 m (275). Por este motivo, actualmente se habla más de un intervalo entre 25 y 35 $\mathrm{m}$, que de un valor puntual (273). En nuestro análisis establecimos $30 \mathrm{~m}$ siguiendo las recomendaciones del documento de consenso sobre pruebas de marcha publicado por la ATS/ERS en 2014 (249). 
Tabla 8a. Características basales de la muestra segmentada según el número de exacerbaciones previas y según el incremento en la distancia del 6MWT tras el entrenamiento.

\begin{tabular}{|c|c|c|c|c|c|c|c|c|c|}
\hline & \multicolumn{2}{|c|}{$\begin{array}{c}\text { Grupo: } \\
0 \text { exacerbaciones } \\
\text { previas } \\
n=15\end{array}$} & \multicolumn{2}{|c|}{$\begin{array}{c}\text { Grupo: } \\
\text { 1-2 exacerbaciones } \\
\text { previas } \\
n=20\end{array}$} & \multicolumn{2}{|c|}{$\begin{array}{c}\text { Grupo: } \\
\Delta \text { Distancia 6MWT } \\
<30 \mathrm{~m} \\
n=5\end{array}$} & \multicolumn{2}{|c|}{$\begin{array}{c}\text { Grupo: } \\
\Delta \text { Distancia 6MWT } \geq \\
30 \mathrm{~m} \\
\mathrm{n}=30\end{array}$} & \\
\hline Sexo & \multicolumn{2}{|c|}{11 hombres (73,3\%) } & \multicolumn{2}{|c|}{16 hombres (80\%) } & \multicolumn{2}{|c|}{5 hombres (100\%) } & \multicolumn{2}{|c|}{22 hombres $(73,3 \%)$} & \\
\hline \multirow{4}{*}{$\begin{array}{c}\text { Clasificación EPOC } \\
\text { (GOLD) }\end{array}$} & \multicolumn{2}{|c|}{4 Grado I (26,7\%) } & \multicolumn{2}{|c|}{1 Grado I (5\%) } & \multicolumn{2}{|c|}{0 Grado I (0\%) } & \multicolumn{2}{|c|}{5 Grado I (16,7\%) } & \\
\hline & \multicolumn{2}{|c|}{1 Grado II (6,7\%) } & \multicolumn{2}{|c|}{7 Grado II (35\%) } & \multicolumn{2}{|c|}{3 Grado II (60\%) } & \multicolumn{2}{|c|}{5 Grado II $(16,7 \%)$} & \\
\hline & \multirow{2}{*}{\multicolumn{2}{|c|}{$\begin{array}{l}1 \text { Grado III (6,7\%) } \\
9 \text { Grado IV (60\%) }\end{array}$}} & \multirow{2}{*}{\multicolumn{2}{|c|}{$\begin{array}{l}3 \text { Grado III (15\%) } \\
9 \text { Grado IV (45\%) }\end{array}$}} & \multirow{2}{*}{\multicolumn{2}{|c|}{$\begin{array}{l}1 \text { Grado III (20\%) } \\
1 \text { Grado IV (20\%) }\end{array}$}} & \multirow{2}{*}{\multicolumn{2}{|c|}{$\begin{array}{c}3 \text { Grado III (10\%) } \\
17 \text { Grado IV (56,7\%) }\end{array}$}} & \\
\hline & & & & & & & & & \\
\hline \multirow{3}{*}{ Tipo de PRR } & \multicolumn{2}{|c|}{2 PRR 1(13,3\%) } & \multicolumn{2}{|c|}{6 PRR 1 (30\%) } & \multicolumn{2}{|c|}{3 PRR 1 (60\%) } & \multicolumn{2}{|c|}{5 PRR 1 (16, 7\%) } & \\
\hline & \multicolumn{2}{|c|}{7 PRR 2(46,7\%) } & \multicolumn{2}{|c|}{9 PRR 2 (45\%) } & \multicolumn{2}{|c|}{1 PRR 2 (20\%) } & \multicolumn{2}{|c|}{15 PRR 2 (60\%) } & \\
\hline & \multicolumn{2}{|c|}{6 PRR 3 (40\%) } & \multicolumn{2}{|c|}{5 PRR 3 (25\%) } & \multicolumn{2}{|c|}{1 PRR $3(20 \%)$} & 10 PRR 3 & $(33,3 \%)$ & \\
\hline Hábito tabáquico & $\begin{array}{r}12 \text { exfur } \\
(80\end{array}$ & ladores & 19 exfumad & ores $(91 \%)$ & 5 exfumadc & res (100\%) & $\begin{array}{r}26 \text { exf } \\
(8 \\
\end{array}$ & $\begin{array}{l}\text { adores } \\
\%)\end{array}$ & ),(a) \\
\hline Uso de OCD & 3 con $\mathrm{OCL}$ & $D(20 \%)$ & $2 \operatorname{con} \mathrm{OCl}$ & D (20\%) & $3 \operatorname{con} 0 c$ & $D(60 \%)$ & $2 \operatorname{con} 0$ & $(6,7 \%)$ & (a) \\
\hline & Media $\pm D E$ & Mín-Max & Media $\pm D E$ & Mín-Max & Media $\pm D E$ & Mín-Max & Media $\pm D E$ & Mín-Max & \\
\hline Edad & $64,1 \pm 12,8$ & $41-79$ & $59,5 \pm 9,5$ & $42-78$ & $56,7 \pm 8,7$ & $43-67$ & $62,2 \pm 11,6$ & $41-79$ & (g),(g) \\
\hline $\begin{array}{l}\text { Evol. enfermedad } \\
\qquad \text { (años) }\end{array}$ & $7,7 \pm 5,9$ & $0-18$ & $5,1 \pm 3,9$ & $0-13$ & $5 \pm 2,6$ & $1-8$ & $6,3 \pm 5,3$ & $0-18$ & $(g),(h)$ \\
\hline $\begin{array}{l}\text { Período sin fumar } \\
\quad \text { (años) }\end{array}$ & $8,1 \pm 5,9$ & $1-18$ & $6,8 \pm 4,1$ & $1-15$ & $4,8 \pm 2,5$ & $1-8$ & $7 \pm 5,2$ & $1-18$ & (g),(h) \\
\hline Paq/año & $51,3 \pm 16,6$ & $20,5-77$ & $39,5 \pm 13,5$ & $18-62$ & $55,5 \pm 14,5$ & $20,5-57,8$ & $45,7 \pm 18,8$ & $18-77$ & (g),(g) \\
\hline IMC $\left(\mathrm{Kg} / \mathrm{m}^{2}\right)$ & $27,1 \pm 5,1$ & $21,3-38,3$ & $26,8 \pm 3,9$ & $21,1-33,5$ & $28 \pm 3,2$ & $24,7-33,3$ & $26,5 \pm 4,5$ & $21,3-38,3$ & (g) \\
\hline $\mathrm{FEV}_{1}(\mathrm{~L})$ & $1,09 \pm 0,35$ & $0,71-1,97$ & $1,29 \pm 0,55$ & $0,68-2,44$ & $1,81 \pm 0,48$ & $1,13-2,22$ & $1,11 \pm 0,41$ & $0,68-2,44$ & (h) \\
\hline $\mathrm{FEV}_{1}(\%)$ & $40,3 \pm 22,2$ & $18,5-77,5$ & $41,5 \pm 16,7$ & $19,4-75,8$ & $45,9 \pm 11,9$ & $28,6-66,1$ & $40,2 \pm 20$ & $18,5-77,5$ & \\
\hline FVC (L) & $2,33 \pm 0,54$ & $0,9-3,17$ & $2,65 \pm 0,74$ & $1,5-3,69$ & $3,45 \pm 0,35$ & $3,03-3,69$ & $2,37 \pm 0,57$ & $0,9-3,51$ & (g),(g) \\
\hline FVC (\%) & $50,6 \pm 13,1$ & $33,4-70,8$ & $51 \pm 10,7$ & $31,9-66,7$ & $57,3 \pm 5$ & $50-62,2$ & $49,8 \pm 12,2$ & $31,9-70,8$ & (g), (g) \\
\hline $\mathrm{FEV}_{1} / \mathrm{FVC}(\%)$ & $43,9 \pm 12,9$ & $27,4-78,5$ & $47,6 \pm 9,1$ & $34,5-69,5$ & $51,8 \pm 9,9$ & $37,3-60,2$ & $45,1 \pm 10,9$ & $27,4-78,5$ & (h),(h) \\
\hline Índice BODE & $3,3 \pm 1,6$ & $0-5$ & $2,9 \pm 1,4$ & $0-6$ & $2,8 \pm 1,9$ & $1-6$ & $3,2 \pm 1,4$ & $0-5$ & (h),(h) \\
\hline
\end{tabular}

Los datos se presentan para la muestra segmentada según el número de exacerbaciones sufridas en los 12 meses previos a recibir los programas de Rehabilitación Respiratoria y según el incremento en la distancia alcanzada en la Prueba de Marcha de Seis Minutos tras el entrenamiento.

Las variables cualitativas sexo, clasificación de la EPOC según los criterios de la Iniciativa GOLD (Global Obstructive Lung Disease) de 2001, tipo de PRR recibido (PRR1, entrenamiento de resistencia; PRR 2, entrenamiento de resistencia-fuerza; PRR 3, entrenamiento de resistencia-fuerza-músculos respiratorios); hábito tabáquico (exfumadores / no fumadores) y la utilización o no de oxigenoterapia continua domiciliaria $(O C D)$, se expresan con sus frecuencias absolutas y relativas (\%).

Para las variables cuantitativas, los datos se exponen como media y desviación estándar (DE) y con el rango (Mín-Máx): Evol. de la enfermedad: años de evolución de la enfermedad desde su diagnóstico; Período sin fumar: años transcurridos desde el cese del hábito tabáquico en los sujetos exfumadores; Paq/año: valor de paquetes/año para los sujetos exfumadores; IMC: índice de masa corporal; FEV : volumen espiratorio forzado

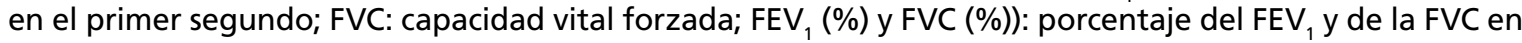
relación al valor teórico.

Se verifica la homogeneidad intergrupo en todas las variables en ambos grupos, evaluado mediante (a) Prueba Exacta de Fisher; (g) T para 2 muestras y $(h) \cup$ de Mann-Whitney $(p>0,05)$. A la izquierda, el test utilizado para el agrupamiento según número de exacerbaciones y a la derecha, para el agrupamiento según la distancia alcanzada en el 6MWT. 
Tabla 8b. Distancia alcanzada en el 6MWT tras el entrenamiento y tras el período de seguimiento para la muestra segmentada según el número de exacerbaciones previas y según el incremento en dicha distancia.

\begin{tabular}{|c|c|c|c|c|c|}
\hline & Pre-Intervención & Post-Intervención & Post-Seguimiento & \multicolumn{2}{|c|}{$\begin{array}{c}\text { Intergrupo } \\
\mathbf{p}\end{array}$} \\
\hline \multicolumn{4}{|c|}{ Segmentación según el número de exacerbaciones previas } & $\begin{array}{c}\text { Post }_{\text {Int }} \\
\text { vs } \\
\text { Pre }_{\text {Int }}\end{array}$ & $\begin{array}{c}\text { Post }_{\text {Seg }} \\
\text { vs } \\
\text { Postl }_{\text {Int }}\end{array}$ \\
\hline 0 exacerbaciones & $407,6 \pm 73,3$ & $478,1 \pm 77,2$ & - & \multirow{2}{*}{$0,029^{(g)}$} & \multirow{2}{*}{-} \\
\hline 1-2 exacerbaciones & $409,1 \pm 59,8$ & $459,6 \pm 73,9$ & - & & \\
\hline \multicolumn{4}{|c|}{ Segmentación según el incremento en la distancia del 6MWT } & & \\
\hline Incremento en la distancia $<30 \mathrm{~m}$ & $367,4 \pm 96,2$ & $378 \pm 85,3$ & $349 \pm 91,2$ & \multirow{2}{*}{$0,000^{(h)}$} & \multirow{2}{*}{$0,034^{(h)}$} \\
\hline Incremento en la distancia $\geq 30 \mathrm{~m}$ & $415,3 \pm 57,6$ & $482,4 \pm 62,8$ & $429,2 \pm 59,8$ & & \\
\hline
\end{tabular}

Los datos se presentan como media y desviación estándar para los tres períodos de medición (previo al inicio de los programas, al finalizar éstos y tras un período de latencia sin mantenimiento) para la variable distancia alcanzada en la Prueba de Marcha de Seis Minutos.

Los valores de p para la comparación intergrupo fueron obtenidos mediante (g) T para 2 muestras y (h) U de Mann-Whitney.

En cuanto a la variación en la distancia del 6MWT post-seguimiento, se observó que el grupo que había mejorado por encima de la distancia mínima clínicamente importante, tuvo una pérdida del $11,1 \%\left(-53,2 \pm 21,3 \mathrm{~m} ; 95 \% \mathrm{IC}\right.$. $\left.(-60,8 ;-46,6) ; p=0,000^{f}\right)$, inferior al $16,6 \%$ de incremento en esta variable tras el entrenamiento $\left(67,1 \pm 19,5 \mathrm{~m} ; 95 \% \mathrm{IC} .(60 ; 74,1) ; p=0,000^{f}\right)$. El otro grupo tuvo una pérdida del $7,7 \%\left(-29 \pm 4,1 \mathrm{~m} ; 95 \% \mathrm{IC}\right.$. $\left.(-50 ;-7,9) ; \mathrm{p}=0,043^{f}\right)$, superior a la mejoría tras el entrenamiento que fue sólo del 2,8\% (10,6 $\pm 12,1 \mathrm{~m} ; 95 \% \mathrm{IC}$. $\left.(0 ; 21,2) ; p=0,080^{\mathrm{e}}\right)$.

Gráfica 8. Efecto del entrenamiento sobre la tolerancia al ejercicio, evaluada mediante la Prueba de Marcha de Seis Minutos, para la muestra segmentada según el número de exacerbaciones sufridas en los 12 meses anteriores al comienzo del programa.

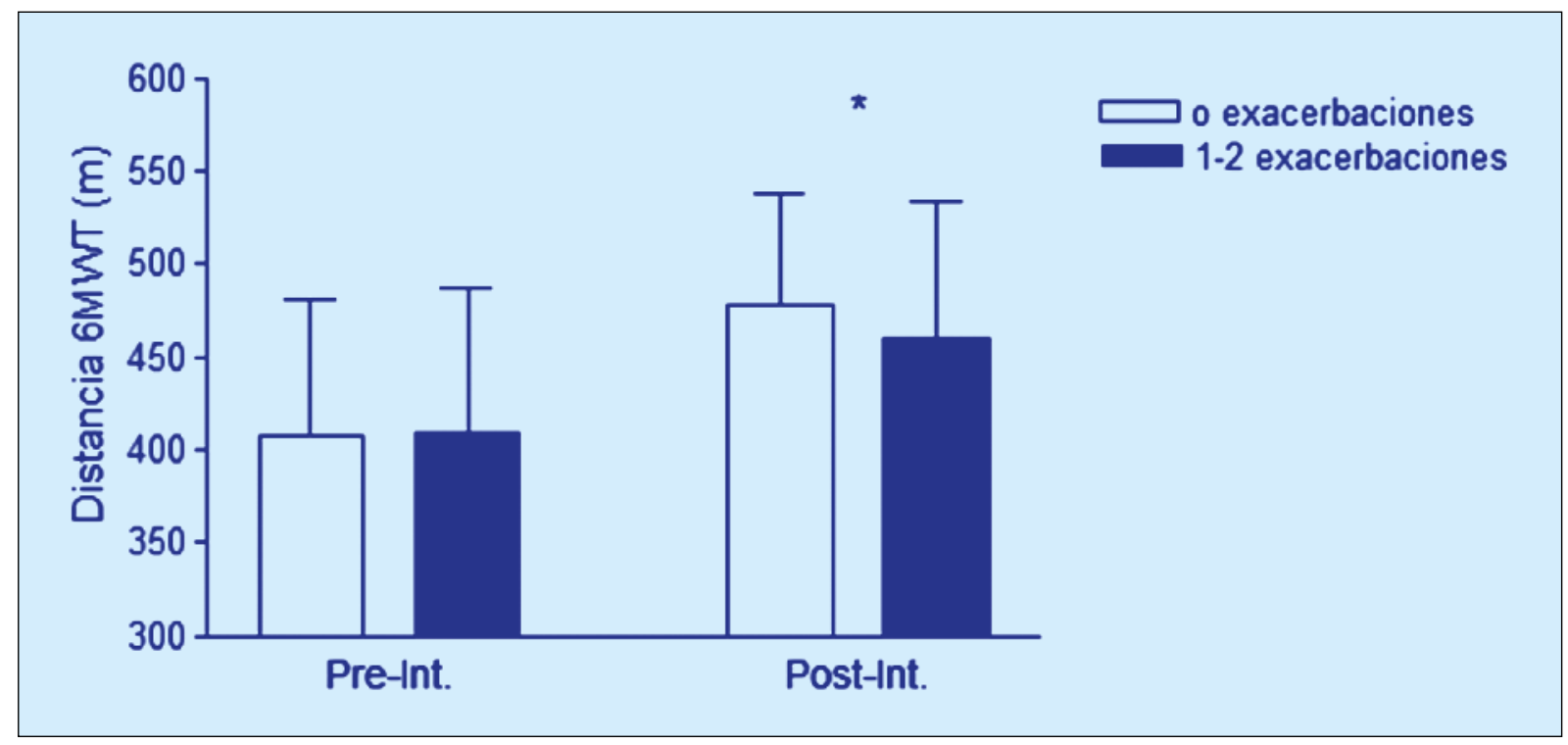

Se representa la media y la desviación estándar de la distancia alcanzada en la Prueba de Marcha de Seis Minutos (6MWT) previo y posterior al entrenamiento (Pre-Int. y Post-Int., respectivamente), para la muestra segmentada según el número de exacerbaciones sufridas en los 12 meses anteriores.

(*), p<0,05 para la comparación entre los grupos post-intervención vs pre-intervención. 
En las Gráficas 8 y 9 se muestran los resultados de la variable distancia alcanzada en el 6MWT post-entrenamiento y post-seguimiento, para la muestra segmentada según el número de exacerbaciones y según el nivel de incremento alcanzado en la prueba de marcha tras la realización de los programas.

\section{Factores de influencia sobre el deterioro en la tolerancia al ejercicio tras el período de seguimiento}

En el cálculo de correlaciones, efectuado controlando por factores de confusión (edad, sexo, IMC, FEV $(\%)$, Índice BODE, uso o no de OCD, horas de ejercicio físico semanal y número de exacerbaciones), sólo se encontraron resultados significativos y relevantes entre la variación en la dinstancia alcanzada en el 6MWT tras el entrenamiento y tras el seguimiento. Se obtuvo una asociación inversa y moderada (coeficiente de correlación de Pearson, r=-0,52 (95\%IC. $(-0,75 ;-0,20) ; p=0,001)$.

En la Gráfica 10 se representa la correlación entre la variación en la distancia del 6MWT tras el entrenamiento y tras el período de seguimiento.

A continuación, con el objetivo de determinar la influencia de la mejoría en la distancia del 6MWT obtenida tras el entrenamiento, sobre el deterioro de esta variable tras el período de seguimiento, se aplicó un modelo de regresión lineal simple. Se verificaron las condiciones de aplicabilidad del modelo mediante el estudio de normalidad de los residuos ( $p=0,462$ para los residuos estandarizados y no estandarizados en el Test de Shapiro-Wilk). El resultado fue estadísticamente significativo $\left(r=0,521 ; r^{2}=0,249 ; p=0,001\right)$, aunque débil

Gráfica 9. Efecto del entrenamiento sobre la tolerancia al ejercicio, evaluada mediante la Prueba de Marcha de Seis Minutos, para la muestra segmentada según el incremento alcanzado en la prueba tras el entrenamiento.

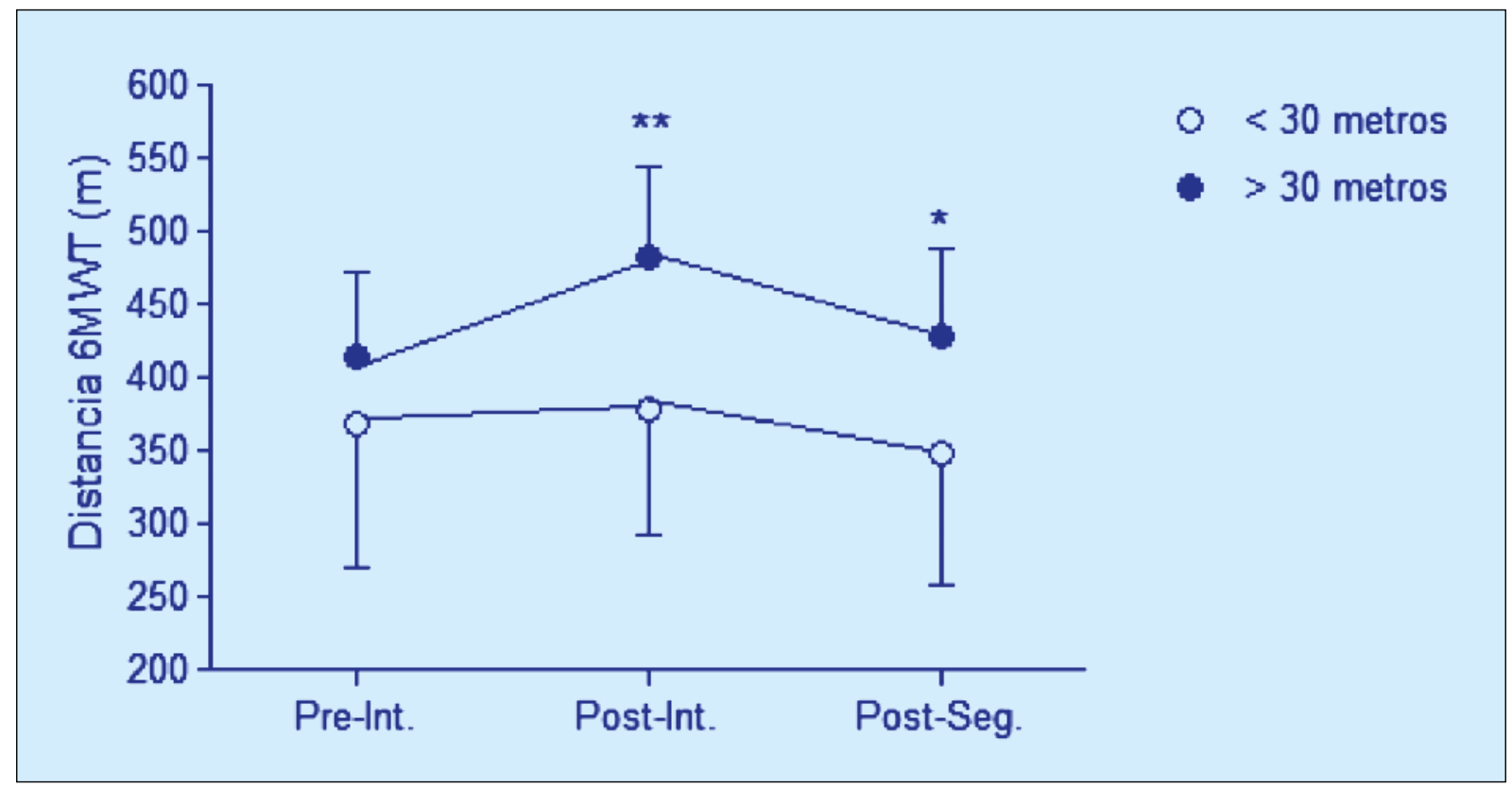

Se representa la media y la desviación estándar de la distancia total alcanzada en la Prueba de Marcha de Seis Minutos (6MWT) en los distintos momentos de medición analizados: Previo al comienzo de los programas (Pre-Int.), a la finalización de los mismos (Post-Int.) y tras un período de seguimiento sin mantenimiento (Post-Seg.). Se muestran los resultados para la muestra segmentada según el incremento obtenido en dicha distancia tras el entrenamiento (no alcanza la distancia mínima clínicamente importante, < 30 metros, o bien, sí alcanza esta distancia, > 30 metros).

$(* *), p<0,01$ y $(*), p<0,05$, para la comparación entre los grupos post-intervención vs pre-intervención y postseguimiento vs pre-intervención. 
$\left(r<0,7\right.$ y $\left.r^{2}<0,5\right)$. La ecuación de predicción obtenida a partir del modelo de regresión fue la siguiente:

$\Delta$ Distancia 6MWT Post-Seg. $(m)=-23,841-(0,439 \times \Delta$ Distancia 6MWT Post-Entr. $)$

Esta ecuación permite predecir la pérdida en la distancia de la prueba de marcha de seis minutos tras un período de entre 9 y 18 meses tras la finalización de los programas de entrenamiento aplicados a la muestra.

Gráfica 10. Asociación entre la variación en la distancia alcanzada en la Prueba de Marcha de Seis Minutos tras el entrenamiento y tras el seguimiento.

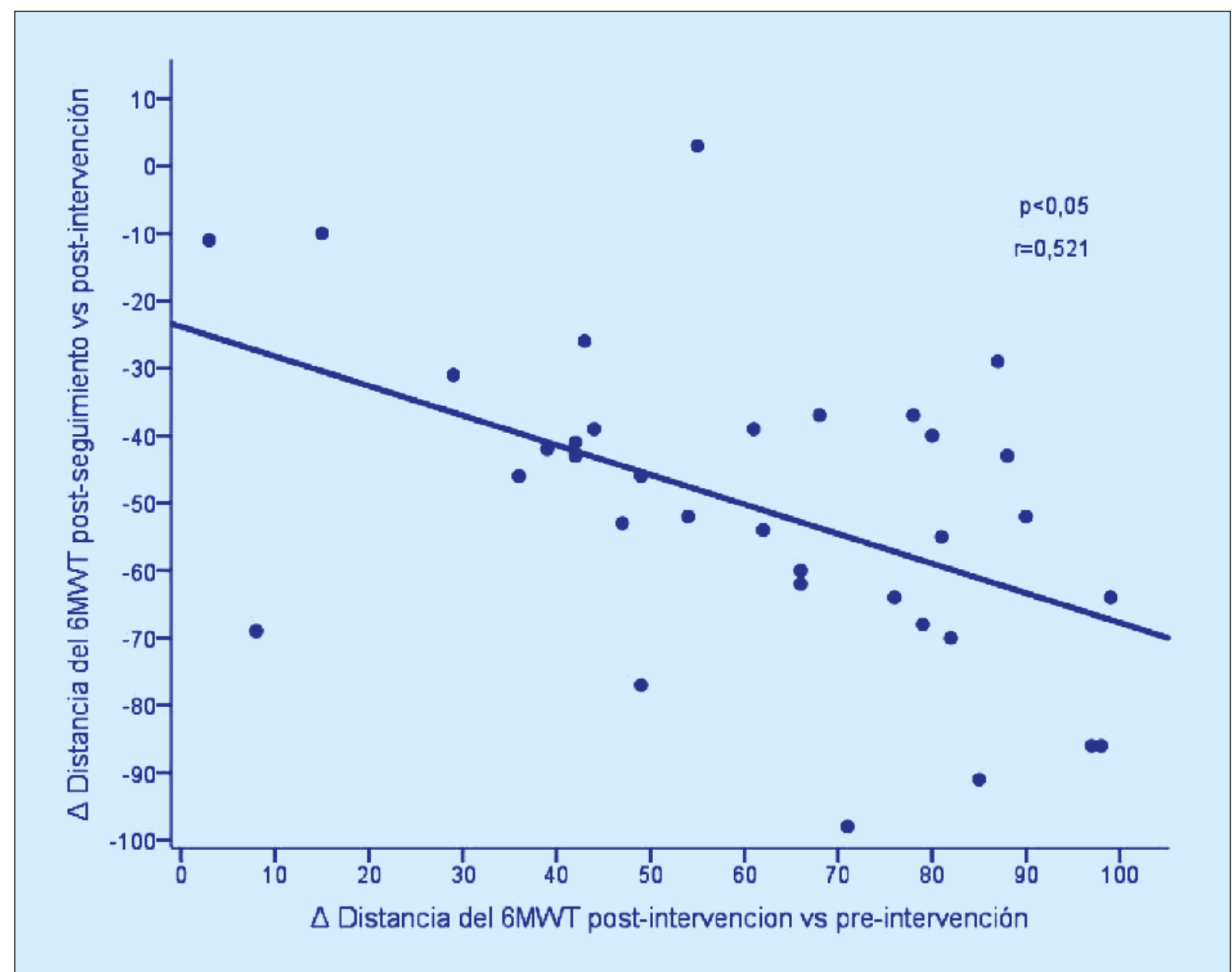

La línea representa la relación existente entre la variación en la distancia alcanzada en la Prueba de Marcha de Seis Minutos (6MWT) tras el entrenamiento (eje $\mathrm{X}$ ) y tras el período de seguimiento (eje $\mathrm{Y}$ ).

(1) Las pruebas utilizadas para el cálculo del valor de $p$ en la comparación itnragupo de las variables descritas en los apartados 2.2. y 3, se indican codificadas dentro de los paréntesis de resultados, siendo: (e),T para datos pareados y (f), Wilcoxon. 


\title{
PARTE III
}

\author{
ESTUDIO 2
}

\section{EFICACIA DE UN PROGRAMA DE ENTRENAMIENTO AUTOADMINISTRADO E INCREMENTO DEL NIVEL DE ACTIVIDAD FÍSICA GUIADO MEDIANTE PODÓMETRO EN PACIENTES CON EPOC}

\section{MATERIAL Y MÉTODOS}

\section{Diseño del estudio}

Ensayo clínico aleatorizado doble ciego, en el que se compara un programa de entrenamiento físico autoadministrado asociado a un plan de incremento del nivel de actividad para pacientes EPOC, con un grupo control que recibe cuidados habituales y recomendación de práctica de ejercicio. El estudio se ha efectuado en la Escuela Universitaria de Fisioterapia de la ONCE (Universidad Autónoma de Madrid) entre 2013 y 2015.

\section{Población de estudio}

La muestra está constituida por 33 pacientes con criterios diagnósticos de EPOC según la Normativa GOLD (43) ( $\mathrm{FEV}_{1}$ inferior al $80 \%$ del valor predictivo, así como una relación $\mathrm{FEV}_{1} /$ FVC menor a 0,7, evaluados en espirometría post-broncodilatación), que cumplían además, los siguientes criterios:

- Estabilidad clínica, sin exacerbaciones en las cuatro semanas previas y con un tratamiento farmacológico adecuadamente pautado y controlado.

- Presentar disnea de esfuerzo.

- Bajo nivel de actividad física habitual, referido por el paciente (menos de 30 minutos diarios de ejercicio físico moderado, como caminar) (2).

Por su parte, fueron excluidos los sujetos que:

- Presentaran enfermedades neurológicas y/o del aparato locomotor, que imposibilitasen la realización de actividad física por parte del paciente.

- Estuvieran diagnosticados de enfermedad cardiovascular concomitante, a excepción de la hipertensión arterial.

- Hubieran participado en un programa de Rehabilitación Respiratoria en los últimos doce meses, previos al estudio.

\section{Muestreo y cálculo del tamaño muestral}

El muestreo se llevó a cabo según un sistema no probabilístico, intencional y de casos consecutivos, incluyendo en el estudio a pacientes susceptibles de formar parte del mismo y que acudieran a consulta en los Servicios de Neumología de los hospitales participantes en el 
proceso de reclutamiento (Hospital Universitario La Paz, Hospital Universitario La Princesa y Fundación Jiménez Díaz), entre los meses de abril de 2013 y marzo de 2014.

El cálculo del tamaño muestral se realizó para la comparación entre dos medias en muestras independientes para las dos variables principales, la tolerancia al ejercicio evaluada mediante una prueba de esfuerzo a carga constante y el nivel de actividad física, evaluado mediante el número de pasos/día. Se consideró una mejoría de 186 segundos (diferencia mínima clínicamente importante) (277) en la prueba de esfuerzo y de 1200 pasos/día (238), así como una desviación estándar de 178 segundos (277) y de 1956 pasos/día (170), respectivamente. Según un riesgo alfa (tipo l) de 0,05 (nivel de confianza del 95\%), un riesgo beta (tipo II) de 0,20 y una tasa de reposición del $20 \%$, se obtuvo un tamaño muestral de 14 sujetos por rama para la variable de tolerancia al ejercicio y de 41 para el nivel de actividad física.

En el protocolo inicial del estudio se estableció que se recuperarían las pérdidas de sujetos ocasionadas por:

- Fallecimiento.

- Aparición de aspectos que imposibilitaran la continuación del individuo en el estudio (algún criterio de exclusión que no se encontraba presente en el inicio).

- Fallos en el proceso de intervención y/o registro de las variables.

No se recuperaron las pérdidas producidas por abandono. Éstas se cuantificaron para el análisis estadístico. Del mismo modo, se consideraron abandono aquellos casos del grupo experimental que incumplieran el programa durante más de tres semanas.

\section{Asignación de los sujetos a los grupos de estudio}

Para la distribución de los sujetos a los grupos, a cada paciente se le asignó un código numérico de cuatro dígitos en orden correlativo según la fecha de ingreso en el proyecto. Cada código había sido previamente asignado al grupo experimental o control mediante aleatorización simple, utilizando un sistema de números aleatorios generados por el programa informático Microsoft@ Excel 2010. La correspondencia entre códigos numéricos y grupo de estudio sólo estuvo disponible a los investigadores encargados de realizar el tratamiento de los pacientes, mientras que los responsables de la medición y registro de las variables desconocían esta información (enmascaramiento de los evaluadores)

\section{Variables}

Para asegurar la calidad de los resultados y reducir los sesgos, la evaluación se realizó por investigadores adiestrados en el manejo de las herramientas de medida y que desconocían si los sujetos pertenecían al grupo experimental o al control. Se entrenó a todas las personas encargadas de la recogida de información antes de comenzar el estudio, con el fin de garantizar que se seguían los mismos procedimientos por todos los miembros del equipo.

Las variables del estudio se registraron en cuatro períodos. En la Tabla 9 se muestra la distribución de las variables recogidas en cada período y a continuación, se describe el procedimiento de medición. 
Tabla 9. Distribución temporal de las variables analizadas.

\begin{tabular}{|c|c|c|}
\hline Registro & Temporalidad & Variables \\
\hline Pre-Intervención & $\begin{array}{c}\text { Semana previa al inicio de la } \\
\text { intervención }\end{array}$ & $\begin{array}{l}\text { Variables de caracterización de la muestra. } \\
\text { Tolerancia al ejercicio (Prueba de Lanzadera a } \\
\text { Carga Constante). } \\
\text { Nivel de actividad física (Cuestionario Baecke } \\
\text { Modificado y pasos caminados por día). } \\
\text { Disnea basal (Escala Modificada del Medical } \\
\text { Research Council). } \\
\text { Calidad de vida (Cuestionario St. George's). } \\
\text { Número de exacerbaciones en los } 12 \text { meses } \\
\text { anteriores. }\end{array}$ \\
\hline Post-Intervención 1 & $\begin{array}{c}\text { A las } 10 \text { semanas del registro } \\
\text { pre-intervención }\end{array}$ & $\begin{array}{l}\text { Tolerancia al ejercicio. } \\
\text { Nivel de actividad física. } \\
\text { Disnea basal. } \\
\text { Calidad de vida. }\end{array}$ \\
\hline Post-Intervención 2 & $\begin{array}{c}\text { A los } 3 \text { meses del registro post- } \\
\text { intervención } 1\end{array}$ & $\begin{array}{l}\text { Tolerancia al ejercicio. } \\
\text { Nivel de actividad física. } \\
\text { Disnea basal. } \\
\text { Calidad de vida. }\end{array}$ \\
\hline Post-Intervención 3 & $\begin{array}{c}\text { A los } 12 \text { meses del registro post- } \\
\text { intervención } 1\end{array}$ & $\begin{array}{l}\text { Tolerancia al ejercicio. } \\
\text { Nivel de actividad física. } \\
\text { Disnea basal. } \\
\text { Calidad de vida. } \\
\text { Número de exacerbaciones en los } 12 \text { meses } \\
\text { anteriores. }\end{array}$ \\
\hline
\end{tabular}

La tabla muestra las variables recogidas en cada uno de los cuatro momentos de registro llevados a cabo durante el estudio.

\subsection{Variables de caracterización de la muestra}

Para la caracterización de la muestra al inicio del estudio, se han analizado las siguientes variables sociodemográficas y clínicas:

- Sexo.

- Edad.

- Evolución de la enfermedad: años transcurridos desde el diagnóstico.

- IMC, en $\mathrm{Kg} / \mathrm{m}^{2}$.

- Hábito tabáquico (fumador/ exfumador/ no fumador), años sin fumar y valor de paquetes/año en los sujetos exfumadores y fumadores.

- Uso de OCD (Sí/No).

- Función pulmonar: $\mathrm{FEV}_{1}, \mathrm{FEV}_{1}(\%), \mathrm{FVC}, \mathrm{FVC}(\%)$ y relación $\mathrm{FEV}_{1} / \mathrm{FVC}$. Se evaluó mediante espirometría forzada, empleando un espirómetro modelo MicroLab (Micromedical; Kent, UK). La prueba se efectuó siguiendo los principios de aplicación descritos en la Normativa SEPAR sobre espirometría, publicada en 2013 (251). 
- Grado de severidad e la EPOC según la clasificación GOLD (43) (Anexo I).

- Puntuación en el Índice BODE (44) (Anexo I).

- Índice de comorbilidades de Charlson (278). Según este índice, una puntuación de 0-1 se considera ausencia de comorbilidades, 2 puntos indica comorbilidad baja y 3 puntos o más, comorbilidad alta.

- Período de participación en el estudio: otoño-invierno (de octubre a marzo) o primavera-verano (de abril a septiembre).

\subsection{Tolerancia al ejercicio}

\section{Prueba de Lanzadera Incremental}

La Prueba de Lanzadera Incremental (268) sólo se realizó en el registro pre-intervención, con el objetivo de extrapolar la velocidad de caminata para la Prueba de Lanzadera a Carga Constante (279), que se utilizaría para evaluar la tolerancia al ejercicio. No obstante, la distancia total alcanzada y el último nivel completado en la prueba, también se utilizaron como variables en la caracterización inicial de la muestra.

El IST es una prueba incremental, estructurada en 12 niveles de dificultad creciente, con un minuto de duración por nivel. El sujeto debe caminar en un circuito de 10 metros a una velocidad prefijada, que va aumentando en cada nivel con respecto al anterior. La regulación de la velocidad de caminata se establece mediante señales acústicas (un pitido simple indica que el sujeto debe encontrarse en uno de los extremos del circuito y un pitido triple marca el cambio de nivel). La prueba finaliza cuando el sujeto no es capaz de caminar a la velocidad o bien, ante la aparición de síntomas.

Para su realización en el estudio se utilizó el procedimiento descrito por la SEPAR (255), acorde con el último establecido por la ATS/ERS (249), efectuando dos pruebas, con un período de descanso de 30 minutos entre ellas y anotando los datos de la de mejores resultados.

Las pruebas se realizaron con oxígeno suplementario a un flujo de 2-3 l/min, en aquellos sujetos con una saturación en reposo inferior al $90 \%$. Así mismo, en los que durante la primera prueba desaturaban por debajo del $85 \%$, la segunda se realizaba con oxígeno.

\section{Prueba de Lanzadera a Carga Constante}

La tolerancia al esfuerzo se evaluó mediante la Prueba de Lanzadera a Carga Constante (279). A pesar de que el gold standard son las pruebas incrementales en cicloergómetro, los test de campo son sencillos, válidos y fácilmente aplicables y aportan información relevante de los cambios tras las intervenciones (280).

La prueba se llevó a cabo siguiendo las directrices establecidas por la SEPAR (255), acorde con el Documento de Consenso sobre Pruebas de Marcha de la ATS/ERS de 2014 (249). El paciente debía caminar a lo largo de un circuito de 10 metros a una velocidad prefijada, que correspondía a un $85 \%$ del $\mathrm{VO}_{2}$ max obtenido en el IST realizado previamente (2-3 días antes). La velocidad se regulaba mediante señales acústicas y se finalizaba la prueba cuando los sujetos eran incapaces de mantener dicha velocidad o bien, ante la aparición de síntomas (disnea o fatiga intolerables, desaturación por debajo del $80 \%$, incremento de la frecuencia cardiaca hasta la frecuencia cardiaca teórica máxima (220 - edad), dolor precordial, mareos, etc.). 
Las pruebas se realizaron con oxígeno suplementario a un flujo de 2-3 l/min en aquellos sujetos con una saturación en reposo inferior al $90 \%$, así como en los que se requirió oxígeno durante el IST previo.

En cada prueba se recogieron las siguientes variables:

- Tiempo total alcanzado.

- Distancia total recorrida.

- Variación durante la prueba (post vs pre) en la disnea percibida y en la fatiga en los MMII, según la Escala de Borg Modificada (253).

- Variación en la FC durante la prueba (post vs pre).

- Variación en la $\mathrm{SaO}_{2}$ durante la prueba (post vs pre). Este parámetro y el anterior se midieron mediante un pulsioxímetro modelo Nonin-8500 (Nonin Medical; Minesota, EEUU).

Se ha determinado la MCID para esta prueba, en 82 metros (281) y 186 segundos (277).

Prueba de Marcha de Seis Minutos

Al igual que el IST, la Prueba de Marcha de Seis Minutos se realizó únicamente al inicio del estudio, con el objetivo de clasificar a los sujetos según el índice BODE (44).

Ésta es una prueba de esfuerzo submáxima en la que el sujeto debe caminar en un circuito recto y sin irregularidades, de al menos 30 metros, durante un tiempo de seis minutos, con el objetivo de alcanzar la máxima distancia posible en dicho período (se debe caminar lo más deprisa posible, sin llegar a correr). A lo largo de la prueba, el individuo puede modificar su ritmo e incluso pararse, en cuyo caso, el cronómetro seguirá transcurriendo y se contabilizará el tiempo total de descanso.

Esta prueba se realizó siguiendo el protocolo establecido por la ATS en 2002 (115), concordante con la actualización de 2014 (249). Cada sujeto debía efectuar dos pruebas con 30 minutos de descanso entre ambas, quedando registrados sólo los datos del test con mejores resultados.

En cuanto a las condiciones de utilización de oxígeno suplementario, fueron las mismas que se han descrito para el IST.

\subsection{Nivel de actividad física}

Los niveles de actividad física se midieron mediante:

- El registro podométrico del número de pasos caminados a lo largo de siete días.

- El Modified Baecke Physical Activity Questionaire (Baecke Modificado).

Se emplearon una herramienta objetiva y otra subjetiva para obtener una información más completa $(153,154)$.

\section{Registro de pasos caminados en siete días}

Se utilizó el podómetro digital OMRON Walking Style $X$ Pocket $\mathrm{HJ}-320 \mathrm{e}$ (Omron Healthcare, Inc.; Illinois, EEUU). Este modelo, de fácil manejo y fiabilidad probada en pacientes con EPOC $(239,246)$ y en sanos $(282,283)$, permitía el registro de los pasos caminados por día y su almacenamiento en la memoria durante siete días. A la hora de elegir el modelo de podómetro, 
buscamos uno que combinara sencillez de manejo, bajo coste y validez para la población de estudio, dado que la fiabilidad de los podómetros varía según el modelo (245).

Los pacientes portaban este dispositivo en el cinturón o cintura del pantalón/falda, a nivel de la cadera derecha. Debían llevarlo durante todo el día, desde que se levantaban hasta que se acostaban, retirándolo sólo en la ducha o si descansaban tumbados, y a lo largo de siete días consecutivos. El objetivo era registrar todos los pasos caminados, incluso en domicilio.

Previo al comienzo del primer período de registro, todos los sujetos realizaban una sesión en la que se les adiestraba en el manejo del dispositivo y se verificaba su correcta utilización. Además, se evaluaba la fiabilidad del podómetro para cada sujeto haciéndole caminar en un circuito de 20 metros durante un minuto a su velocidad habitual y con el podómetro activado; simultáneamente, un observador externo contabilizaba los pasos que daba el sujeto y posteriormente, se contrastaba el registro podométrico con el conteo manual. Se estableció excluir a aquellos sujetos en que la variabilidad entre ambas medidas superara un $10 \%$. Sistemas similares de verificación de la fiabilidad del dispositivo en la muestra de estudio han sido utilizados previamente $(170,239,284)$.

Los sujetos debían anotar el número total de pasos que marcaba el podómetro al final de cada día durante el período de registro y proporcionar estos datos en la consulta de evaluación. A su vez, el evaluador contrastaba el registro recogido por cada sujeto con los datos almacenados en la memoria del dispositivo. Se estableció descartar aquellos registros con una diferencia superior al $10 \%$ entre los dos sistemas. En los registros válidos, pero con ligeras discrepancias, se utilizó como dato válido el almacenado en la memoria del podómetro.

En el análisis se utilizó el promedio de pasos/día, excluyendo los días en que el registro de pasos fue inferior a 200, considerándose días sin medición válida (170).

\section{Cuestionario de Actividad Física Baecke Modificado}

El Baecke Modificado es un cuestionario específico para evaluar las actividades de la vida diaria en individuos de más de 60 años, validado para pacientes EPOC en español (285). Su elección vino determinada por ser considerado uno de los cuestionarios más fiables para evaluar AF en este tipo de pacientes (153).

El cuestionario debe ser cumplimentado mediante entrevista personal y consta de tres dimensiones: actividades caseras, de ocio y actividad física y deportiva. En cada dimensión se contempla la intensidad y el tiempo promedio de cada actividad. De la puntuación obtenida se extrae el grado de sedentarismo del entrevistado, mediante una puntuación específica ( $<9$ puntos, sedentarismo; 9-16, nivel de actividad física moderada; $>16$ puntos, individuo activo) (285).

\subsection{Calidad de vida}

La calidad de vida se midió mediante el St. George's Respiratory Questionaire, validado y específico para medir calidad de vida relacionada con la salud en pacientes con EPOC (286). En concreto, se empleó la versión adaptada al español (287).

El SGRQ es un cuestionario autoadministrado constituido por 50 ítems distribuidos en tres dimensiones: síntomas relacionados con la enfermedad (8 ítems), actividad diaria y sus limitaciones producidas por la disnea (16 ítems) e impacto de la enfermedad en la esfera psicosocial (26 ítems). 
La puntuación obtenida en el cuestionario va de 0 a 100. A mayor valor, má impacto de la enfermedad en la calidad de vida. Una diferencia de 4 puntos se considera clínicamente significativa (288).

\subsection{Disnea basal}

Para evaluar la percepción de disnea basal por parte de los pacientes, se empleó la Escala Modificada del Medical Research Council (254). Se trata de una escala numérica de cinco niveles (del 0 al 4), cada uno de los cuales se corresponde con una definición breve de la sensación de disnea percibida. El sujeto debe indicar al entrevistador, a qué nivel se asemeja más su sensación de disnea habitual en las actividades de la vida cotidiana.

\subsection{Número de exacerbaciones}

Se recogió el número de exacerbaciones que había sufrido cada paciente en los 12 meses anteriores al momento del registro. Se consideró episodio de exacerbación al empeoramiento en al menos dos de los síntomas habituales (disnea, cantidad de expectoración y color del esputo) durante más de 24 horas (252), con necesidad de tratamiento antibiótico y/o ingreso hospitalario.

\section{Intervención}

Todos los pacientes derivados al proyecto pasaron una entrevista en la que se establecía o no el cumplimiento de los criterios de inclusión. Aquellos individuos que aceptaban integrarse en el estudio, tras haber sido informados del propósito del mismo y firmar el consentimiento informado (Anexo IV), eran asignados aleatoriamente a un grupo de estudio y comenzaban el protocolo correspondiente. En la Figura 29 se resume, mediante un diagrama de flujo, el protocolo completo del estudio.

La intervención en ambos grupos fue llevada a cabo por una fisioterapeuta especializada en Fisioterapia Respiratoria. Ésta se dividió en dos partes, siendo la primera parte, común a ambos grupos de estudio.

\subsection{Primera parte}

Esta fase incluía cinco sesiones de Fisioterapia Respiratoria de carácter educativo, en las que se adiestraba a los pacientes en el desarrollo de las siguientes técnicas:

- Reeducación ventilatoria (Figuras 6, 7, 8, 9 y 10): Ventilación dirigida diafragmática y costal (257), ventilación con labios fruncidos (258), etc. El objetivo era optimizar la ventilación de los pacientes, instruirles en el empleo habitual de un patrón ventilatorio eficaz y reducir el atrapamiento aéreo (259). Las técnicas empleadas en cada caso dependían de la situación clínica de cada paciente, evitando el adiestramiento diafragmático en pacientes con hiperinsuflación importante, tal y como indican las guías de práctica clínica $(78,81,260)$. No se consideró la influencia de estas técnicas en los resultados del estudio, ya que no se ha demostrado que su aplicación en el contexto de la RR produzca ningún efecto sobre la capacidad de ejercicio, la disnea o la calidad de vida (261).

- Drenaje de secreciones (Figura 11): Se llevaban a cabo técnicas como la ELTGOL (262) y las TEF (263), así como el adiestramiento de una tos eficaz. La decisión de emplear unas técnicas u otras o ninguna de ellas, venía determinada por los hallazgos de una auscultación pulmonar previa, que se practicaba al inicio de la sesión a todos los pacientes. 


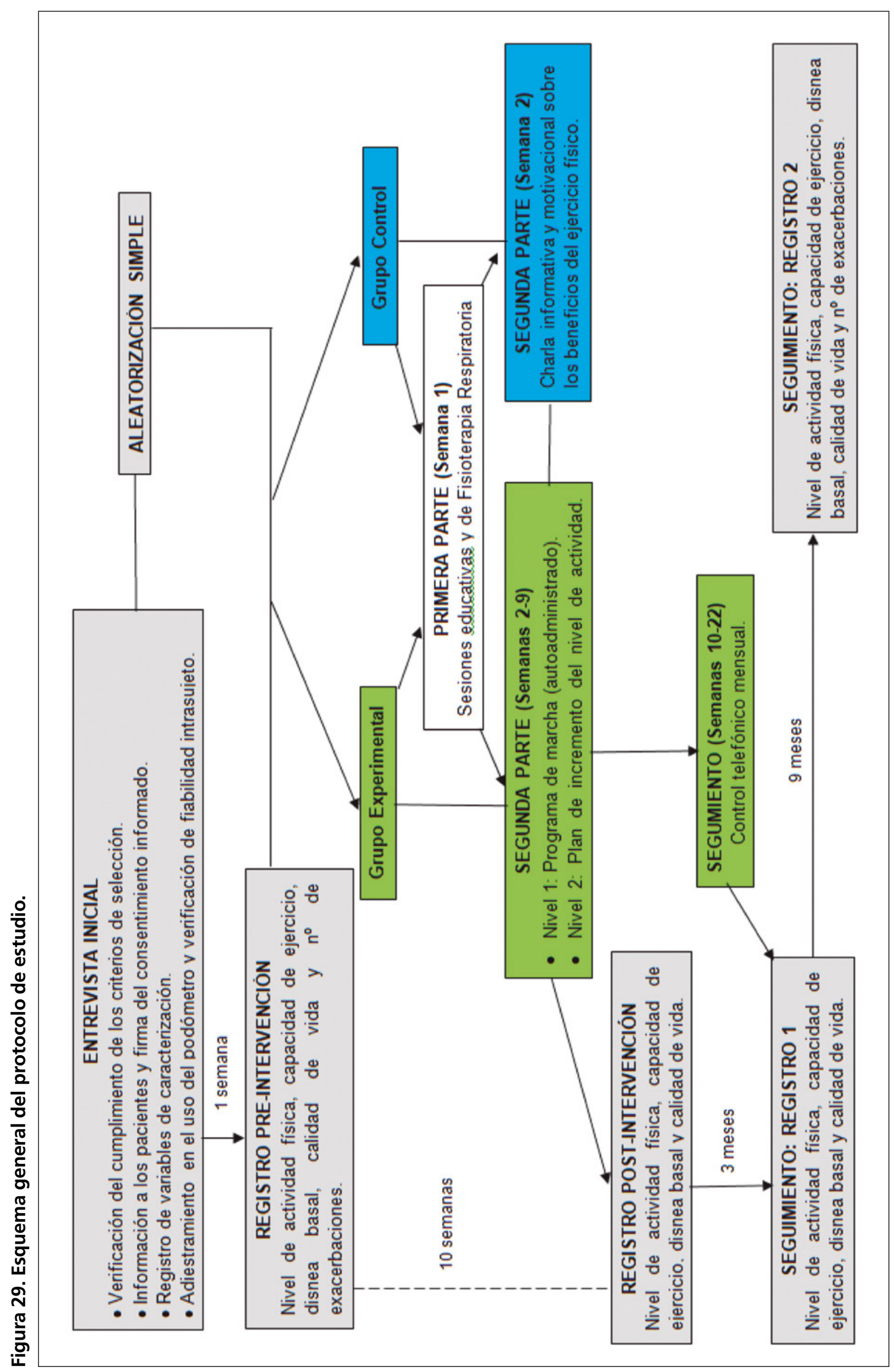


Todas las técnicas seguían los principios de aplicación que se describen en el Manual de Procedimientos publicado por SEPAR en 2013, sobre técnicas manuales e instrumentales para el manejo de secreciones bronquiales en el adulto (266).

- Movilización del tórax: Ejercicios de cinesiterapia activa libre del tórax combinados con respiración, con el objetivo de flexibilizar la caja torácica (Figuras 15, 16 y 17).

- Adiestramiento en el manejo de los dispositivos de inhalación. Se enseñó a los pacientes la técnica de administración de fármacos inhalados mediante inhaladores presurizados, cámaras espaciadoras e inhaladores de polvo seco, y se comprobó su correcta utilización (según las directrices establecidas por el Consenso SEPAR y la Asociación Latinoamericana del Tórax de 2013) (289).
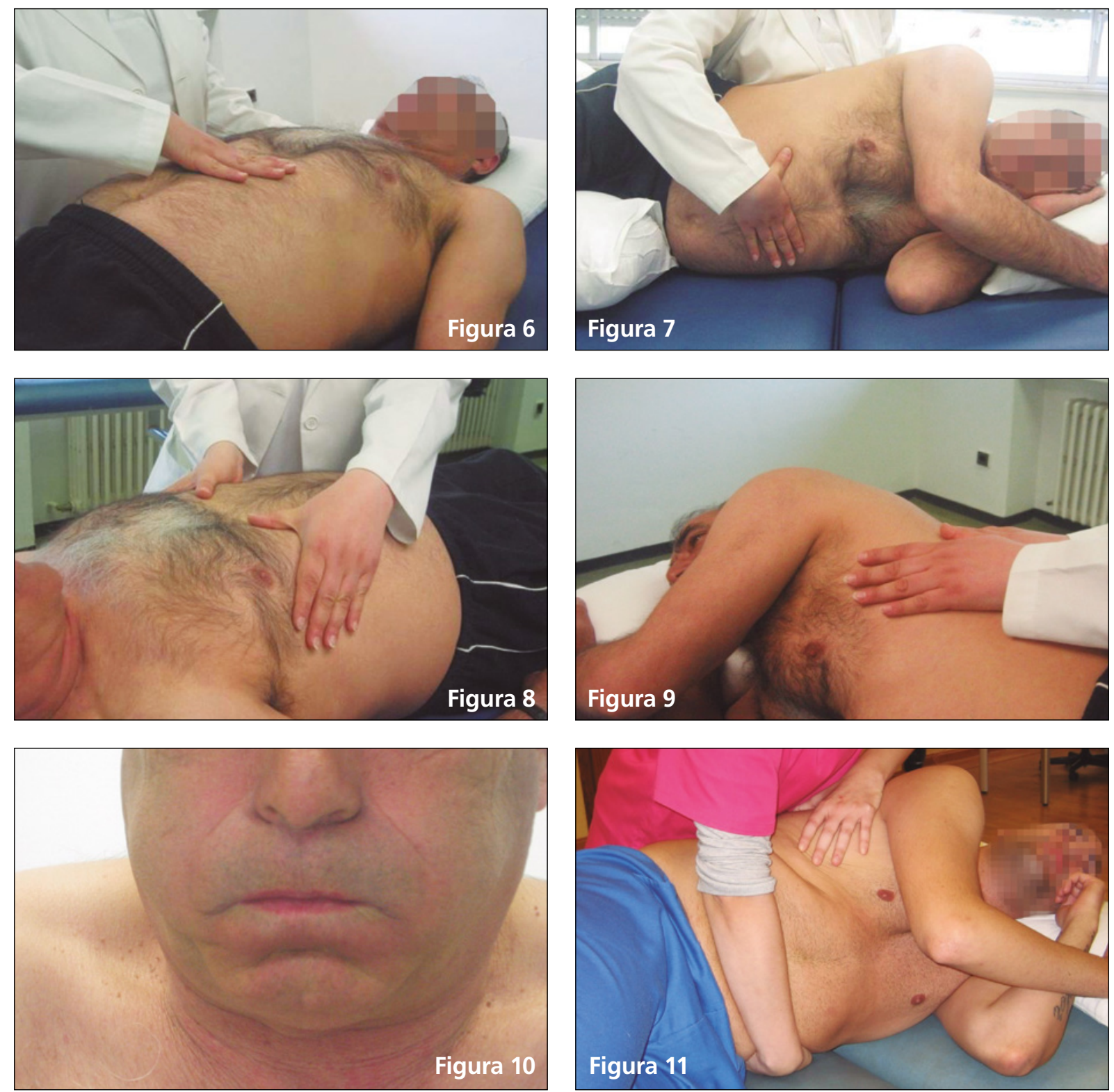

Figura 6. Técnica de ventilación dirigida diafragmática en posición de decúbito supino.

Figura 7. Técnica de ventilación dirigida diafragmática en posición de decúbito lateral.

Figura 8. Técnica de ventilación dirigida costal basal bilateral.

Figura 9. Técnica de ventilación dirigida costal basal unilateral.

Figura 10. Ventilación con "labios fruncidos".

Figura 11. Técnica de ELTGOL. 
Las sesiones se llevaban a cabo de forma grupal (3-4 pacientes máximo), en días consecutivos, con una hora de duración para cada sesión y aplicando de forma individualizada cada técnica en función de las necesidades de cada paciente, a criterio del fisioterapeuta responsable, pero siguiendo los consensos mínimos que se acordaron previamente por el equipo. Estas sesiones grupales se programaron de tal modo que no hubo contacto entre los pacientes del grupo experimental y el control, con el fin de garantizar el enmascaramiento de los sujetos, que en todo momento desconocían en que consistía la intervención en el otro grupo.
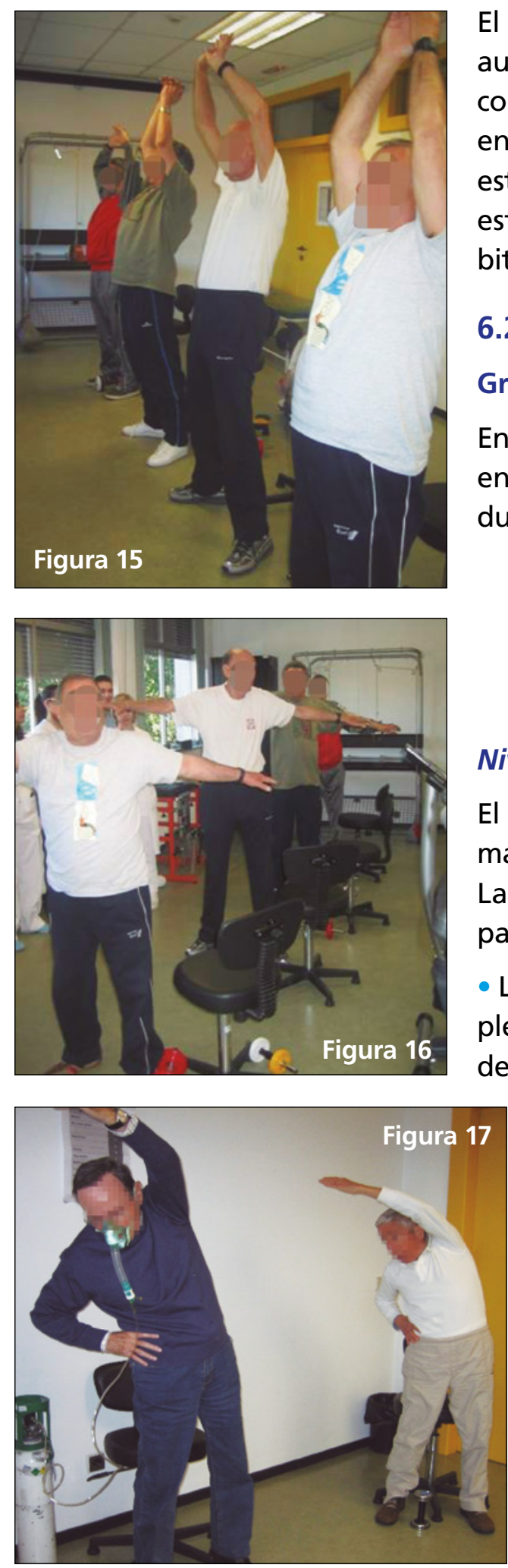

El objetivo de esta primera parte de la intervención fue aumentar la adherencia de los pacientes al proyecto, así como mejorar el control consciente y el manejo de su enfermedad. No se pretendía en ningún momento que esta actuación tuviera otro efecto sobre la tolerancia al esfuerzo o la modificación de conducta en cuanto a hábitos de práctica de ejercicio físico se refiere.

\subsection{Segunda parte}

\section{Grupo experimental}

En el grupo experimental se efectuó un programa de entrenamiento autoadministrado de ocho semanas de duración, desarrollado a dos niveles:

- Nivel 1: Entrenamiento físico mediante un programa de marcha progresivo.

- Nivel 2: Plan de incremento de la actividad física diaria.

\section{Nivel 1: Programa de marcha}

El entrenamiento consistía en caminar cinco días por semana durante un tiempo y a una velocidad prefijadas. La progresión del programa se estableció en base a dos parámetros (Tabla 10):

- La velocidad, que se basaba en la del último nivel completado en el IST realizado en la exploración inicial. Se ha demostrado que este valor corresponde a la denominada "velocidad crítica", a la que se alcanza un $90 \%$ del $\mathrm{VO}_{2}$ max, siendo éste el nivel máximo de esfuerzo sostenido (290). En el programa, comenzaban caminando al $80 \%$ de dicha velocidad e iba incrementándose este parámetro hasta el $110 \%$, a partir de la cuarta semana.

- El tiempo de caminata: Se comenzaba por $30 \mathrm{mi}-$ nutos diarios, en dos ciclos de 15 minutos cada uno

Figura 15. Ejercicios de flexibilización del tórax en flexo-extensión.

Figura 16. Ejercicios de flexibilización del tórax en rotación.

Figura 17. Ejercicios de flexibilización del tórax en lateralidad. 
Tabla 10. Progresión del programa de marcha aplicado al grupo experimental.

\begin{tabular}{|c|c|c|c|c|}
\hline Semana & Días/ semana & $\begin{array}{l}\text { Tiempo total de } \\
\text { caminata/ día }\end{array}$ & Ciclos de caminata/ día & $\mathrm{N}^{\circ}$ pasos/ ciclo \\
\hline 1 & 5 & $30 \mathrm{~min}$. & 2 ciclos de $15 \mathrm{~min}$. & $0,8 \times \mathrm{n} \times \mathrm{t}$ \\
\hline 2 & 5 & $30 \mathrm{~min}$. & 2 ciclos de $15 \mathrm{~min}$. & $0,9 \times n \times t$ \\
\hline 3 & 5 & $30 \mathrm{~min}$. & 2 ciclos de $15 \mathrm{~min}$. & $\mathrm{n} \times \mathrm{t}$ \\
\hline 4 & 5 & $40 \mathrm{~min}$. & 2 ciclos de 20 min. & $\mathrm{n} \times \mathrm{t}$ \\
\hline 5 & 5 & $40 \mathrm{~min}$. & 2 ciclos de $20 \mathrm{~min}$. & $1,1 \times n \times t$ \\
\hline 6 & 5 & $50 \mathrm{~min}$. & 1 ciclo de $20 \mathrm{~min} .+2$ ciclos de $15 \mathrm{~min}$. & $1,1 \times n \times t$ \\
\hline 7 & 5 & $55 \mathrm{~min}$. & 2 ciclos de $20 \mathrm{~min} .+1$ ciclo de $15 \mathrm{~min}$. & $1,1 \times n \times t$ \\
\hline 8 & 5 & $60 \mathrm{~min}$. & 3 ciclos de $20 \mathrm{~min}$. & $1,1 \times n \times t$ \\
\hline
\end{tabular}

La tabla muestra la progresión del programa, semana a semana, en cuanto al tiempo total de entrenamiento diario y su distribución en ciclos. Así mismo se describe el número de pasos que debían conseguir durante cada ciclo de entrenamiento, siendo n el número de pasos alcanzados en 1 minuto caminando a la velocidad del último nivel completado en la Prueba de Lanzadera (velocidad crítica) y t la duración de cada ciclo.

y se iba incrementando hasta alcanzar 60 minutos en 3 ciclos de 20 minutos, durante la octava semana. El tiempo de entrenamiento se fragmentó en períodos más cortos para garantizar que todos los sujetos pudieran cumplir las pautas de intensidad, puesto que se sabe que ancianos y pacientes crónicos, hasta que se consigue efecto entrenamiento, pueden no soportar intensidades elevadas durante 30 minutos seguidos (88).

Para el control de la velocidad de marcha, los pacientes utilizaban un podómetro OMRON Walking Style $X$ Pocket HJ-320e (Omron Healthcare, Inc.; Illinois, EEUU). Consideramos que el podómetro era una herramienta eficaz para la monitorización durante el entrenamiento, puesto que se ha demostrado su elevada fiabilidad caminando a velocidades cercanas a la alcanzada en un 6MWT (245), que era similar a la de entrenamiento en el programa.

Se calculaba previamente el número de pasos que cada sujeto daba en un minuto caminando a la velocidad de entrenamiento y a partir de ahí, se extrapolaba el número de pasos que debían alcanzar en cada ciclo de caminata. Este control indirecto permitía a los pacientes, regular su velocidad para ajustarla al valor óptimo.

Los pacientes podían realizar sus caminatas en el momento del día y el lugar que prefiriesen (recomendándoles que evitaran las horas posteriores a las ingestas alimentarias, así como las de más calor del día, en caso de períodos veraniegos).

La duración del programa y la frecuencia de las sesiones de entrenamiento fueron consistentes con las recomendaciones de las principales guías de práctica clínica $(47-49,72,81,83)$. Se diseñó un entrenamiento a alta intensidad, puesto que el ejercicio por encima del $80 \%$ del Wmax consigue mejores resultados en la tolerancia al esfuerzo (291) y entrenamientos por debajo del $60 \%$ no alcanzan adaptaciones musculares (88). No se añadió entrenamiento de fuerza ni ningún otro tipo de ejercicio aeróbico para reducir la complejidad del programa y evitar así, incumplimientos o abandonos. El entrenamiento mediante marcha era sencillo y de fácil realización.

\section{Nivel 2: Plan de incremento del nivel de actividad física}

Además del programa de marcha, se estableció otra actuación destinada a modificar los hábitos de actividad física, incrementando progresivamente el número de pasos caminados 
a lo largo de todo el día. Esta intervención de cambio comportamental, se llevó a cabo simultáneamente al programa de entrenamiento y se inspiró en la "Teoría de establecimiento de metas u objetivos", descrita por Locke (234), basada en:

- La fijación de una meta clara y alcanzable.

- El establecimiento de objetivos parciales que permitan llegar, progresivamente, a la meta final.

- El compromiso de los sujetos en llevar a cabo las acciones necesarias para la consecución de los objetivos marcados.

- El empleo de sistemas de feed-back que permitan al individuo conocer el nivel de alcance de los objetivos.

- La retroalimentación por parte del profesional y el establecimiento de nuevos objetivos.

Basándonos en estas premisas, desarrollamos un plan que consistía en lo siguiente:

- Durante las ocho semanas del programa, los pacientes debían llevar consigo el podómetro, desde que se levantaban hasta que se acostaban. Al final de cada jornada, anotaban en un diario de actividad (Anexo $\mathrm{V}$ ) el número de pasos caminados a lo largo de todo el día, diferenciando los realizados en los ciclos de entrenamiento de los efectuados durante el resto del día.

- Cada semana se marcaba como objetivo aumentar en un $10-20 \%$ el número total de pasos con respecto a la semana anterior, excluyendo en la cuantificación los realizados durante las caminatas del programa de marcha. En la primera semana se tomaba como referencia el número de pasos obtenidos en la medición pre-intervención.

- Se llevaba a cabo un control telefónico semanal para supervisar el cumplimiento del programa. En estas llamadas se solicitaba el registro de pasos, se analizaba con cada paciente las posibles causas de incumplimiento y se pautaba el nuevo objetivo para la semana siguiente. En las conversaciones se intentaba mantener un ambiente positivo y motivador para favorecer la adherencia al programa

Consideramos que el podómetro podía ser una herramienta muy útil para incentivar a los pacientes en una actividad tan simple y accesible como es caminar. La visualización directa de un dato objetivo, como es el número de pasos, facilitaría el control de cuál es la "meta" a alcanzar en días consecutivos.

Previo a comenzar el programa, cada paciente recibía una sesión individualizada de adiestramiento en la velocidad óptima de marcha durante los ciclos de entrenamiento y en el registro de actividad en el diario.

Al finalizar el programa, se indicó a los pacientes que debían mantener el nivel de actividad física alcanzado en la última semana. Se les recomendó que continuaran utilizando el podómetro como sistema de feed-back.

Durante los tres meses siguientes, se mantuvo un control telefónico mensual, aunque se suprimió la obligatoriedad del registro sistemático de los pasos diarios. Estas llamadas telefónicas tenían como objetivo actuar de recordatorio de la importancia de permanecer activos, animando y motivando a los pacientes a continuar con los hábitos adquiridos en el programa. 


\section{Grupo control}

Los pacientes integrantes del grupo control solamente recibían una charla acerca de los beneficios del ejercicio físico, en la que se insistía en la importancia de incrementar el nivel de actividad física habitual. Se les proporcionó el mismo podómetro que a los sujetos del grupo experimental, pero no se aportaron pautas específicas ni recomendaciones de uso.

\section{Almacenamiento de los datos}

Todos los datos fueron recogidos inicialmente en unas fichas (Anexos VI y VII) y posteriormente, almacenados en una hoja de cálculo elaborada para tal fin, empleando el programa informático Microsoft@ Excel 2010.

A lo largo del estudio se desarrollaron mecanismos de verificación de los datos y de control de calidad, con el objetivo de garantizar en todo momento que la información se recogía de acuerdo a lo establecido y que se seguían en todos los procesos, las normas acordadas.

\section{Análisis estadístico}

Para el análisis estadístico se utilizó el paquete informático SPSS® Statistics versión 20.0 (SPSS Incorporation; Chicago, EEUU).

Se llevó a cabo un análisis descriptivo para todas las variables en la muestra segmentada según el grupo de intervención. Para las cualitativas se calcularon las frecuencias absolutas y relativas de cada una de sus categorías. Para las cuantitativas se obtuvieron medidas de tendencia central (media) y de dispersión (desviación estándar), así como el rango. Se analizó la homogeneidad de los grupos al inicio del estudio mediante la prueba Exacta de Fisher y la prueba de Chi-cuadrado (variables cualitativas), la prueba T para 2 muestras y la prueba U de Mann-Whitney (variables cuantitativas, según distribución normal o no).

Se realizó un análisis comparativo de los resultados post-entrenamiento vs pre-entrenamiento y post-seguimiento (a los 3 y a los 12 meses) vs pre-entrenamiento, para cada grupo de estudio, reportando la media, la desviación estándar, el intervalo de confianza al 95\% y el porcentaje de variación observado, así como el valor de $\mathrm{p}$ calculado a partir de pruebas paramétricas ( $T$ para datos pareados) y no paramétricos (Wilcoxon). Así mismo, se analizó la existencia de diferencias significativas entre los grupos en estos resultados, mediante la prueba T para 2 muestras y la prueba $U$ de Mann-whitney (según distribución de la muestra). En aquellas variables en que se obtuvieron diferencias significativas entre los grupos, se aplicó un análisis de covarianza para determinar el efecto de la intervención ajustado según valores basales (previa verificación de las condiciones de aplicabilidad del modelo de regresión mediante el análisis de normalidad de los residuos utilizando el Test de Shapiro-Wilk).

El procedimiento anterior siguió un análisis por protocolo. No obstante, para reducir sesgos, se llevó a cabo un análisis de sensibilidad por intención de tratar en las variables principales, tolerancia al esfuerzo y nivel de actividad física.

Posteriormente, se evaluó la existencia de diferencias en los resultados observados en cada grupo de estudio, según el sexo, la edad (hasta 60 años o mayor de 60 años), el nivel de severidad de la EPOC según clasificación GOLD (43), el período estacional en el que se desarrolló el programa (otoño-invierno o primavera-verano) y el uso de OCD. Se aplicó el mismo procedimiento descrito anteriormente, para grupo control y experimental por separado, analizando las diferencias intra e intergrupo en los niveles de segmentación indicados. 
Por último, se calcularon los coeficientes de correlación de Pearson y Spearman (según distribución de la muestra) para determinar la asociación entre la modificación en el nivel de actividad física de los sujetos y los cambios producidos en la tolerancia al ejercicio, la calidad de vida, la disnea basal y el número de exacerbaciones, durante el período de seguimiento en relación a los niveles previos a la intervención. El análisis se realizó controlando por los factores de confusión que en la literatura se describen como influyentes sobre el nivel de actividad física (edad, $\mathrm{FEV}_{1}$, comorbilidades, Índice BODE, distancia alcanzada en el 6MWT) $(169,172,174,188,198)$ y por los valores basales delas variables resultado.

Para el análisis de normalidad de todas las variables incluidas en el estudio se utilizó la prueba de Shapiro-Wilk. En todos los análisis se asumió un nivel de confianza $(1-\alpha)$ de 0,95 (nivel de significación $\alpha=0,05$ ). Así mismo, se especifica cuando se producen resultados muy significativos (nivel de significación $\alpha=0,01$ ).

\section{Consideraciones éticas}

El equipo investigador ha tenido en cuenta los aspectos éticos a la hora de diseñar el proyecto, contemplando los principios de beneficencia, respeto y justicia. El desarrollo del proyecto se fundamentó en las Declaraciones de la Asociación Médica Mundial del Helsinki (1964) y en las revisiones posteriores, así como en el Código Deontológico de la Asociación de Colegios Médicos de España.

Las actuaciones que se han llevado a cabo respetaron siempre las reglas de buena práctica clínica y tuvieron como exclusiva finalidad, la investigación clínica con el objeto de mejorar la asistencia sanitaria. A los sujetos participantes en el estudio no se les sometió a ningún procedimiento innecesario y tampoco dejaron de recibir las atenciones necesarias en el tratamiento de su enfermedad. El protocolo experimental no tuvo implicaciones nocivas o riesgos, más bien al contrario.

En relación a la selección de la muestra, como ya se ha descrito, se siguieron una serie de principios totalmente compatibles con la ética científica:

- Distribución aleatoria en los grupos.

- Información completa del desarrollo del estudio.

- Garantía del derecho a la intimidad y la privacidad de sus datos, en los términos establecidos en la Ley 15/1999 de Protección de Datos de Carácter General y en la Ley 41/2000, reguladora de la autonomía del paciente y de derechos y obligaciones en materia de información y documentación clínica. Se utilizaron códigos numéricos que se asignaron a cada sujeto, empleando éstos para su identificación en lugar de sus datos personales.

- Posibilidad de abandonar el proyecto en cualquier momento sin dar explicaciones y sin que por ello se viera afectado el tratamiento de los mismos.

- Inclusión de los individuos únicamente tras la firma del consentimiento informado.

Este proyecto fue aprobado por cada uno de los Comités de Ética e Investigación Clínica de los hospitales colaboradores en el reclutamiento de los sujetos (Hospital Universitario La Paz (Cod. PI-1244), Hospital Universitario La Princesa (Cod. PI-484) y Fundación Jiménez Díaz (Cod. $\mathrm{PI}-46)$ ). 


\section{RESULTADOS}

\section{Características de la población de estudio}

Se reclutó un total de 40 pacientes, de los que fueron excluidos $7(17,5 \%)$ por abandono o incumplimiento de los programas. De éstos, 21 fueron asignados al grupo experimental y 19 al control, 31 eran hombres y 9 mujeres, con edades comprendidas entre 41 y 79 años. Los 7 sujetos excluidos eran varones, 4 de ellos pertenecían al grupo experimental y 3 al control. En las Tablas 11a y 11b se presentan las características basales de la muestra según el grupo de estudio.

Durante el proceso de verificación de la fiabilidad del registro de pasos mediante el podómetro, en ningún caso se produjo una variabilidad en relación al conteo manual superior al $10 \%$, por lo que ningún sujeto fue excluido del estudio por esta causa. Tampoco por discrepancias entre los datos recogidos por los sujetos y el registro almacenado en el podómetro, así como no se produjeron pérdidas o abandonos durante el período de seguimiento.

Finalmente, completaron el estudio 33 sujetos (16 en el grupo control y 17 en el experimental), 24 hombres y 9 mujeres, con una edad comprendida entre los 47 y los 79 años. En la Figura 30 se muestra un diagrama de flujo con la evolución de los sujetos participantes desde el reclutamiento hasta la finalización del estudio.

\section{Efectos de las intervenciones a corto, medio y largo plazo'}

\subsection{Análisis pro protocolo}

Post-intervención vs pre-intervención (Tabla 12)

Tabla 11a. Características basales de la población de estudio (variables sociodemográficas).

\begin{tabular}{|c|c|c|c|c|}
\hline & \multicolumn{2}{|c|}{$\begin{array}{l}\text { Grupo experimental } \\
\qquad n=21\end{array}$} & \multicolumn{2}{|c|}{$\begin{array}{l}\text { Grupo control } \\
\qquad n=19\end{array}$} \\
\hline Sexo & \multicolumn{2}{|c|}{18 hombres $(85,7 \%)$} & \multicolumn{2}{|c|}{13 hombres $(68,4 \%)$} \\
\hline Período & \multicolumn{2}{|c|}{15 en octubre-marzo $(71,6 \%)$} & \multicolumn{2}{|c|}{14 en octubre-marzo $(73,7 \%)$} \\
\hline \multirow[t]{2}{*}{ Hábito tabáquico } & \multicolumn{2}{|c|}{$\begin{array}{c}1 \text { fumadores }(4,8 \%) \\
17 \text { exfumadores }(81 \%) \\
3 \text { no fumadores }(14,3 \%)\end{array}$} & \multicolumn{2}{|c|}{$\begin{array}{c}2 \text { fumadores }(10,5 \%) \\
15 \text { exfumadores }(78,9 \%) \\
2 \text { no fumadores }(10,5 \%)\end{array}$} \\
\hline & Media $\pm D E$ & Mín-Max & Media $\pm D E$ & Mín-Max \\
\hline Edad & $69,5 \pm 7,4$ & $47-78$ & $64,8 \pm 9,1$ & $41-79$ \\
\hline Período sin fumar (años) & $15,5 \pm 13,3$ & $0-42$ & $11,6 \pm 12,3$ & $0-40$ \\
\hline Paq/año & $38,7 \pm 23,3$ & $0-78$ & $39,9 \pm 29,1$ & $0-122$ \\
\hline IMC $\left(\mathrm{Kg} / \mathrm{m}^{2}\right)$ & $26,3 \pm 4,9$ & $18,3-41,6$ & $26,6 \pm 5$ & $20-38,3$ \\
\hline
\end{tabular}

Las variables cualitativas sexo, período de participación en el estudio (de octubre a marzo / de abril a septiembre) y hábito tabáquico se expresan con sus frecuencias absolutas y relativas (\%).

Para las variables cuantitativas, los datos se exponen como media y desviación estándar (DE) y con el rango (Mín-Máx): Período sin fumar: años transcurridos desde el cese del hábito tabáquico en los sujetos exfumadores; Paq/año: valor de paquetes/año para los sujetos fumadores y exfumadores; IMC: índice de masa corporal.

Se verifica la homogeneidad intergrupo en todas las variables, evaluada mediante (a) Prueba Exacta de Fisher, (i) Chi-Cuadrado, (g) T para 2 muestras y (h) U de Mann-Whitney ( $p>0,05)$. 


\section{Tolerancia al ejercicio}

En el grupo experimental se produjo una mejora en los resultados del EST, tanto en el tiempo total de la prueba $\left(6,1 \pm 5,7 \mathrm{~min} ; 95 \% \mathrm{IC} .(3,3 ; 8,9) ; p=0,000^{\circ}\right)$ como en la distancia total recorrida $\left(491,4 \pm 486,5 \mathrm{~m} ; 95 \% \mathrm{IC}\right.$. $\left.(232,5 ; 714,3) ; \mathrm{p}=0,000^{\circ}\right)$. Esto supuso un incremento del $\approx 103 \%$ en el tiempo y del $\approx 90 \%$ en la distancia. Por su parte, en el grupo control no se observaron diferencias significativas tras la intervención, ni en el tiempo total alcanzado en la prueba $\left(-0,9 \pm 2,1 \mathrm{~min} ; 95 \%\right.$ IC. $\left.(-1,9 ; 0,1) ; p=0,152^{\mathrm{e}}\right)$ ni en la distancia $(-70,3 \pm 163,4 \mathrm{~m} ; 95 \% \mathrm{IC} .(-153,7 ;$ $\left.13,1) ; p=0,152^{e}\right)$. Estas diferencias entre los grupos fueron estadísticamente significativas. Sin embargo, el resto de variables recogidas en la prueba (disnea, fatiga, frecuencia cardiaca y saturación de oxígeno) no sufrieron cambios.

\section{Nivel de actividad física}

En relación a la puntuación total obtenida en el Cuestionario Baecke Modificado también presentó diferencias significativas entre los grupos. En el grupo experimental se incrementó

Tabla 11b. Características basales de la población de estudio (variables clínicas).

\begin{tabular}{|c|c|c|c|c|}
\hline & \multicolumn{2}{|c|}{$\begin{array}{l}\text { Grupo experimental } \\
\qquad n=21\end{array}$} & \multicolumn{2}{|c|}{$\begin{array}{c}\text { Grupo control } \\
n=19\end{array}$} \\
\hline Clasificación EPOC (GOLD) & \multicolumn{2}{|c|}{$\begin{array}{c}7 \text { Grado II }(33,3 \%) \\
11 \text { Grado III }(52,4 \%) \\
3 \text { Grado IV }(14,3 \%)\end{array}$} & \multicolumn{2}{|c|}{$\begin{array}{l}11 \text { Grado II (57,9\%) } \\
6 \text { Grado III (31,6\%) } \\
2 \text { Grado IV (10,5\%) }\end{array}$} \\
\hline \multirow[t]{2}{*}{ Uso de OCD } & \multicolumn{2}{|c|}{3 con OCD $(14,3 \%)$} & \multicolumn{2}{|c|}{3 con OCD $(15,8 \%)$} \\
\hline & Media $\pm D E$ & Mín-Max & Media $\pm D E$ & Mín-Max \\
\hline Evol. enfermedad (años) & $10,2 \pm 7,4$ & $0-27$ & $8 \pm 6,9$ & $0-30$ \\
\hline $\mathrm{FEV}_{1}(\mathrm{~L})$ & $1,44 \pm 0,48$ & $0,64-2,75$ & $1,52 \pm 0,6$ & $0,59-2,88$ \\
\hline $\mathrm{FEV}_{1}(\%)$ & $45,8 \pm 16,5$ & $16,7-76$ & $52,3 \pm 15,7$ & $23,8-75,5$ \\
\hline FVC (L) & $2,51 \pm 0,55$ & $1,86-3,94$ & $2,57 \pm 0,88$ & $1,36-4,26$ \\
\hline FVC (\%) & $50,7 \pm 13,3$ & $31,2-72,9$ & $55,3 \pm 16,9$ & $38,7-73$ \\
\hline $\mathrm{FEV}_{1} / \mathrm{FVC}(\%)$ & $55,5 \pm 10,6$ & $32,8-69,8$ & $54,9 \pm 15,6$ & $39,6-68,6$ \\
\hline Índice BODE & $2,3 \pm 1,4$ & $0-5$ & $2 \pm 1,2$ & $1-5$ \\
\hline Índice de Charlson & $2,7 \pm 2,7$ & $1-10$ & $1,5 \pm 0,9$ & $1-4$ \\
\hline 6 MWT - Distancia (m) & $462 \pm 125,2$ & $175-690$ & $496,7 \pm 125,3$ & $308-725$ \\
\hline IST - Último nivel & $5,9 \pm 1,5$ & $2-9$ & $6,3 \pm 1,6$ & $4-9$ \\
\hline IST - Distancia (m) & $353,8 \pm 107,9$ & $110-660$ & $397,4 \pm 140$ & $180-650$ \\
\hline
\end{tabular}

Las variables cualitativas clasificación de la EPOC según los criterios de la Iniciativa GOLD (Global Obstructive Lung Disease) 2001 y utilización o no de oxigenoterapia continua domiciliaria (OCD), se expresa con sus frecuencias absoluta y relativas (\%).

Para las variables cuantitativas, los datos se exponen como media y desviación estándar (DE) y con el rango

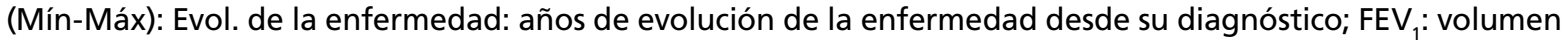
espiratorio forzado en el primer segundo; FVC: capacidad vital forzada; FEV 1 (\%) y FVC (\%)): porcentaje del $\mathrm{FEV}_{1}$ y de la FVC en relación al valor teórico; 6MWT: Distancia alcanzada en la Prueba de Marcha de Seis Minutos; IST: Prueba de Lanzadera Incremental, último nivel completado y distancia total alcanzada.

Se verifica la homogeneidad intergrupo en todas las variables, evaluada mediante (a) Prueba Exacta de Fisher, (g) T para 2 muestras y (h) U de Mann-Whitney ( $p>0,05)$. 
Figura 30. Diagrama de flujo de participación CONSORT (Consolidated Standards of Reporting Trials)

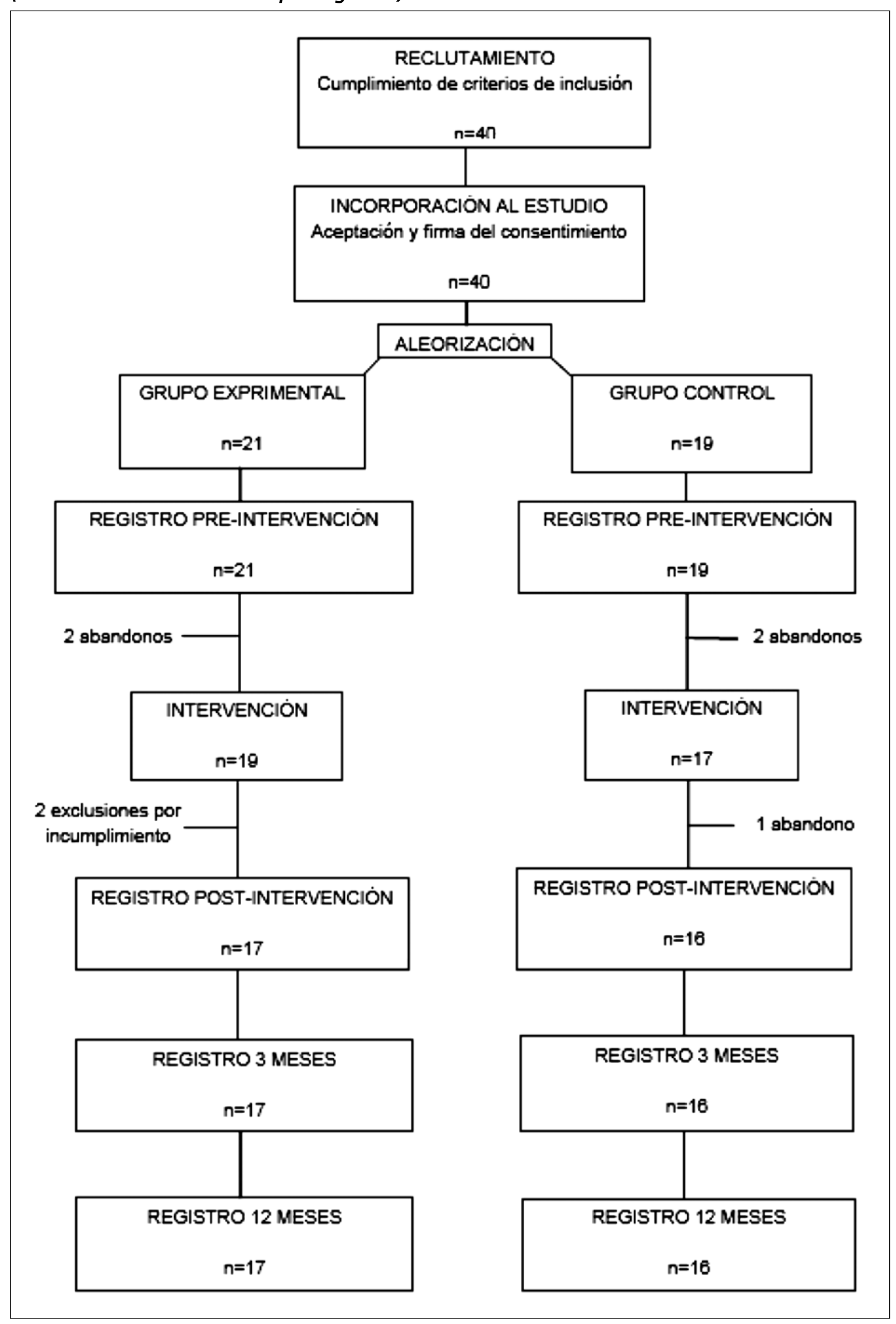


en un $\approx 28 \%(1,7 \pm 2,2$ puntos; $95 \%$ IC. $(0,6 ; 2,8) ; p=0,006)$, mientras que en el grupo control no acontecieron apenas cambios $\left(0 \pm 1,4\right.$ puntos; $\left.95 \% I C(-0,7 ; 0,7) ; p=0,790^{\mathrm{e}}\right)$. En el análisis de las puntuaciones obtenidas para cada dimensión del cuestionario (actividades domésticas, ocio y deporte), no se obtuvieron diferencias significativas entre los grupos.

Por su parte, en relación a los pasos/día, en el grupo experimental se produjo un incremento del $\approx 45 \%$ (3.158 \pm 2.191 pasos/día; 95\%IC. (2.092; 4.264); $\left.p=0,000^{\circ}\right)$. Sin embargo, en el grupo control se observó una ligera reducción, no significativa (-163 \pm 2.795 pasos/día; 95\%IC. (-1.590; 1.264); $\left.p=0,819^{f}\right)$. Estas diferencias intergrupo fueron estadísticamente significativas.

\section{Calidad de vida y disnea basal}

En el grupo experimental se redujo la puntuación total del SGRQ en un $\approx 11 \%(-4,4 \pm 3,6$ puntos; $95 \%$ IC. $\left.(-6,2 ;-2,6) ; p=0,000^{f}\right)$, así como la correspondiente a la dimensión de impacto de

Tabla 12. Efectos inmediatos tras la realización de los programas.

\begin{tabular}{|c|c|c|c|c|c|}
\hline & \multicolumn{2}{|c|}{$\begin{array}{l}\text { Grupo experimental } \\
\qquad \mathrm{n}=17\end{array}$} & \multicolumn{2}{|c|}{$\begin{array}{c}\text { Grupo control } \\
n=16\end{array}$} & \multirow{2}{*}{$\begin{array}{c}\begin{array}{c}\text { Inter } \\
\text { grupo }\end{array} \\
p\end{array}$} \\
\hline & Pre-Interv. & Post-Interv. & Pre-Interv. & Post-Interv. & \\
\hline EST - Tiempo (min) & $5,9 \pm 4,5$ & $12 \pm 8,4$ & $8,4 \pm 5.4$ & $7,5 \pm 6,1$ & $0,000^{(h)}$ \\
\hline EST - Distancia (m) & $588,7 \pm 576,3$ & $1.062 \pm 759,9$ & $702,2 \pm 559,7$ & $631,9 \pm 623,4$ & $0,000^{(g)}$ \\
\hline EST - $\Delta$ Disnea (Borg) & $4,3 \pm 2,6$ & $3,3 \pm 2,2$ & $4,7 \pm 2$ & $4,3 \pm 2,1$ & $0,168^{(g)}$ \\
\hline EST - $\Delta$ Fatiga (Borg) & $1,8 \pm 1,5$ & $1,2 \pm 0,9$ & $2,2 \pm 1,8$ & $2,4 \pm 2,1$ & $0,179^{(h)}$ \\
\hline EST - $\Delta$ FC (lpm) & $39,3 \pm 13,6$ & $33,8 \pm 15,3$ & $39,9 \pm 15,8$ & $36,2 \pm 20,7$ & $0,620^{(g)}$ \\
\hline EST - $\Delta$ SatO $_{2}(\%)$ & $-5,2 \pm 5,1$ & $-4,5 \pm 4,1$ & $-6,8 \pm 6,5$ & $-6,9 \pm 4,8$ & $0,453^{(g)}$ \\
\hline Pasos/día & $7.121 \pm 3.689$ & $10.299 \pm 4.392$ & $5.777 \pm 2.112$ & $5.615 \pm 3.136$ & $0,000^{(\mathrm{h})}$ \\
\hline Baecke - Total & $6,3 \pm 3$ & $8 \pm 4,2$ & $4,6 \pm 2,7$ & $4,6 \pm 2,7$ & $0,002^{(h)}$ \\
\hline Baecke - Act.Domésticas & $1,3 \pm 0,8$ & $1,4 \pm 0,8$ & $1,7 \pm 0,6$ & $1,7 \pm 0,6$ & $0,449^{(g)}$ \\
\hline Baecke - Ocio & $2,3 \pm 1,5$ & $2,3 \pm 1,7$ & $1,1 \pm 0,6$ & $1,1 \pm 0,8$ & $0,857^{(\mathrm{h})}$ \\
\hline Baecke - Deporte & $3,9 \pm 2,9$ & $5,3 \pm 3,5$ & $1,7 \pm 2,6$ & $1,7 \pm 2,8$ & $0,087^{(\mathrm{h})}$ \\
\hline SGRQ - Total & $41,2 \pm 13,2$ & $36,9 \pm 12,5$ & $44,6 \pm 15,6$ & $45,6 \pm 14,5$ & $0,000^{(\mathrm{h})}$ \\
\hline SGRQ - Síntomas & $38,2 \pm 13,2$ & $34,2 \pm 16,6$ & $44,1 \pm 21,6$ & $47,9 \pm 23,4$ & $0,024^{(\mathrm{h})}$ \\
\hline SGRQ - Actividad & $56,1 \pm 20,5$ & $53,8 \pm 19,1$ & $59,8 \pm 18,3$ & $61,5 \pm 20,1$ & $0,058^{(h)}$ \\
\hline SGRQ - Impacto & $31,5 \pm 15,1$ & $25,7 \pm 12,6$ & $36,3 \pm 15,5$ & $37,7 \pm 15,2$ & $0,000^{(h)}$ \\
\hline Disnea basal (mMRC) & $1,4 \pm 0,8$ & $1,1 \pm 0,7$ & $1,5 \pm 0,7$ & $1,5 \pm 0,5$ & $0.444^{(\mathrm{h})}$ \\
\hline
\end{tabular}

Los datos se presentan como media y desviación estándar para los registros pre-intervención (Pre-Interv.) y post-intervención inmediata (Post-Interv.), para cada grupo de estudio.

EST - Tiempo (min), EST -Distancia (m), EST - $\Delta$ Disnea (Borg), EST - $\Delta$ Fatiga (Borg), EST - $\Delta$ FC (lpm), EST - $\Delta$ $\mathrm{SatO}_{2}(\%)$ : tiempo en minutos, distancia en metros, variación en la disnea y fatiga según Escala de Borg modificada, en la frecuencia cardiaca y en la saturación de oxígeno, durante la Prueba de Lanzadera a Carga Constante (EST); Pasos/día: número de pasos al día registrados durante siete días consecutivos; Baecke Total, Baecke - Act.Domésticas, Baecke - Ocio y Baecke - Deporte: puntuación total y de cada una de sus dimensiones en el Cuestionario de Actividad Física Baecke Modificado; SGRQ - Total, SGRQ - Síntomas, SGRQ - Actividad y SGRQ - Impacto: puntuación total y de cada una de las dimensiones del Cuestionario de Calidad de Vida St. George's; Disnea basal (mMRC): disnea basal según Escala Modificada del Medical Research Council.

Los valores de p para la comparación entre los grupos de estudio fueron obtenidos mediante (g) T para 2 muestras y (h) U de Mann Whitney. 
la enfermedad en un $\approx 18 \%(-5,8 \pm 5,2$ puntos; $95 \% \mid C .(-8,4 ;-3,2) ; p=0,000)$. Por su parte, estas puntuaciones aumentaron ligera aunque no significativamente, en el grupo control $((1 \pm 4,3$ puntos; $95 \%$ IC. $(-1,2 ; 3,2) ; p=0,349 f)$ para la puntuación total y $(1,4 \pm 5,6$ puntos; $95 \%$ IC. $(-1,5$; $4,3) ; p=0,315^{f}$ ) para la dimensión de impacto). Estas diferencias entre los grupos fueron estadísticamente significativas, mientras que no se observaron cambios en las dimensiones de síntomas y actividad.

En relación a la disnea, en el grupo experimental se produjo una ligera reducción en la puntuación de la Escala mMRC $\left(-0,2 \pm 0,4 ; 95 \% \mathrm{IC} .(-0,4 ; 0) ; p=0,046^{f}\right)$, mientras que en el grupo control no se observaron cambios $\left(0 \pm 0,6 ; 95 \% \mathrm{IC}\right.$. $\left.(-0,3 ; 0,3) ; p=1,000^{\mathrm{e}}\right)$. En la comparación intergrupo, estas diferencias no resultaron estadísticamente significativas.

A los 3 y 12 meses tras la intervención vs pre-intervención (Tabla 13)

\section{Tolerancia al ejercicio}

Durante el seguimiento, el grupo experimental mantuvo la mejoría alcanzada en los resultados del EST. A los 3 meses en relación al registro pre-intervención, el incremento era del $\approx 112 \%$ en el tiempo total de la prueba $\left(6,9 \pm 6,7 \mathrm{~min} ; 95 \%\right.$ IC. $\left.(3,4 ; 10,3) ; p=0,001^{\mathrm{e}}\right)$ y del $\approx 94 \%$ en la distancia $\left(592,6 \pm 595,6 \mathrm{~m} ; 95 \% \mathrm{IC}\right.$. $\left.(256,4 ; 858,9) ; p=0,000^{\mathrm{e}}\right)$. A los 12 meses, el aumento del tiempo era del $\approx 95 \%(5,5 \pm 6,3 \mathrm{~min} ; 95 \% \mathrm{IC}$. $(2,3 ; 8,8) ; \mathrm{p}=0,001 \mathrm{e})$ y del $\approx 81 \%$ en la distancia $(472 \pm 576,6 \mathrm{~m} ; 95 \% \mathrm{IC}$. $(175,6 ; 768,5) ; p=0,001 \%)$. Por su parte, en el grupo control se produjo un empeoramiento significativo, tanto a los 3 meses $\left(\left(-1,4 \pm 2,4\right.\right.$ min; $\left.95 \% I C . ~(-2,7 ; 0) ; p=0,030^{\circ}\right)$ en el tiempo y $(-57,3 \pm 262,1 \mathrm{~m} ; 95 \%$ IC. $(-197 ; 82,3) ; p=0,014$ e) en la distancia), como a los $12 \mathrm{me}-$ ses $\left(\left(-2 \pm 2,2 \mathrm{~min} ; 95 \%\right.\right.$ IC. $\left.(-2,2 ;-0,8) ; p=0,000^{c}\right)$ en el tiempo y $(-152,4 \pm 164,9 \mathrm{~m} ; 95 \%$ IC. $(-240,3$; $-64,5) ; p=0,000^{\mathrm{e}}$ ) en la distancia). Estas diferencias entre los grupos fueron estadísticamente significativas, mientras que en el resto de variables recogidas en la prueba, sólo se detectaron cambios significativos en la disnea a los 3 meses (reducción en el grupo experimental, ver Tabla 13).

En la Gráfica 11 se representan el tiempo y la distancia alcanzados en el EST a lo largo del estudio, para cada grupo.

\section{Nivel de actividad física}

En la primera parte del período de seguimiento, el grupo experimental continuó aumentando el número de pasos/día, y posteriormente, comenzó un ligero declive, aunque a los 12 meses aún se mantenían valores superiores al basal. A los 3 meses, el aumento en relación al registro pre-intervención era del $\approx 49 \%$ (3.525 \pm 2.404 pasos/día; $95 \%$ IC. $\left.(2.289 ; 4.761) ; \mathrm{p}=0,000^{\mathrm{e}}\right)$ y a los 12 meses, del $\approx 25 \%$ (1.797 \pm 2.947 pasos/día; $95 \%$ IC. (2.82; 3.312); $\left.\mathrm{p}=0,023^{\mathrm{f}}\right)$. Por su parte, en el grupo control se observó un descenso no significativo a los 3 meses $(-316 \pm 2.333$ pasos/ día; $95 \%$ IC. (-1.559; 926$) ; p=0,556$ ) y a los 12 meses $(-750 \pm 1.659$ pasos/día; 95\%IC. (-1.634; 134$)$; $\left.\mathrm{p}=0,091^{\mathrm{f}}\right)$. Las diferencias entre grupos fueron estadísticamente significativas.

En cuanto a la puntuación total del Cuestionario Baecke Modificado, continuó aumentando significativamente en el grupo experimental, $\approx 52 \%$ a los 3 meses $(3,3 \pm 3,1$ puntos; $95 \%$ IC. $\left.(1,7 ; 4,9) ; p=0,000^{f}\right)$ y $\approx 33 \%$ a los 12 meses $\left(2 \pm 2,6\right.$ puntos; $\left.95 \% I C .(0,7 ; 3,3) ; p=0,005^{f}\right)$. El grupo control no presentó cambios ni a los 3 meses $\left(-0,1 \pm 0,6\right.$ puntos; $95 \%$ IC. $\left.(-0,7 ; 0,9) ; p=0,248^{e}\right)$ ni a los 12 meses $(-0,2 \pm 0,8$ puntos); $95 \% \mid C$. $(-0,6 ; 0,2) ; p=0,099)$. Estas diferencias intergrupo fueron estadísticamente significativas. Sin embargo, en el análisis diferenciado de las dimensiones del cuestionario, sólo se observaron diferencias significativas en la dimensión de actividades deportivas a los 3 meses, con un incremento del $\approx 36 \%$ en el grupo experimental 


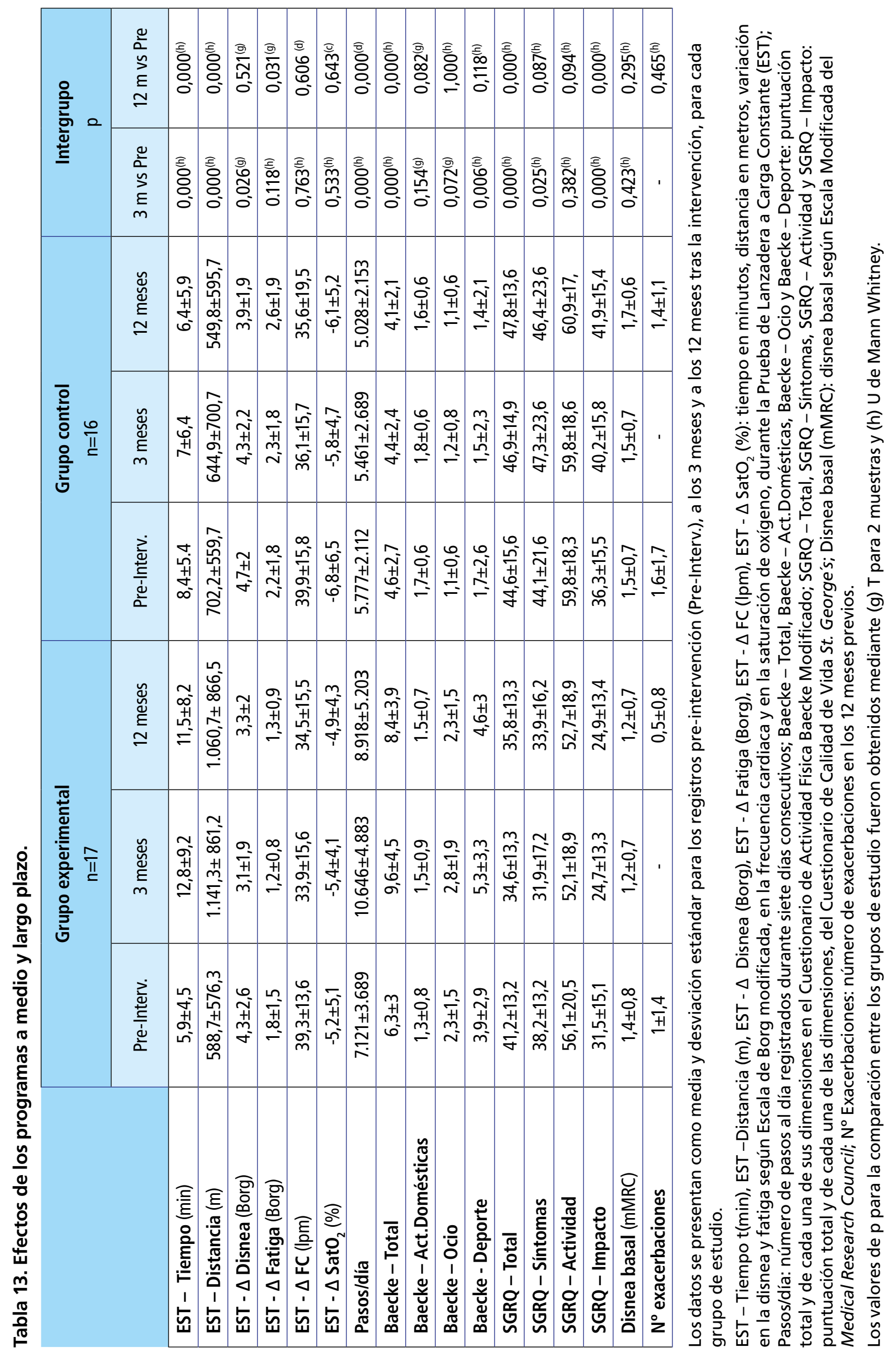


$(1,4 \pm 2$ puntos; $95 \%$ IC. $(0,4 ; 2,5) ; p=0,024)$ frente a la ausencia de cambios en el grupo control $(-0,1 \pm 1,5$ puntos; $95 \%$ IC. $(-0,9 ;-0,7) ; p=0,499)$.

En la Gráfica 12 se representa el número de pasos/día y la puntuación total del Cuestionario Baecke Modificado para los grupos a lo largo del estudio.

\section{Calidad de vida, disnea y exacerbaciones}

Las puntuaciones total y de la dimensión de impacto del SGRQ se mantuvieron con tendencia decreciente durante el período de seguimiento en el grupo experimental. Se observó una reducción del $\approx 16 \%$ en la puntuación total y del $\approx 22 \%$ en la dimensión de impacto, a los 3

Gráfica 11. Efecto de los programas sobre la tolerancia al ejercicio evaluada mediante la Prueba de Lanzadera a Carga Constante (Endurance Shuttle Test).

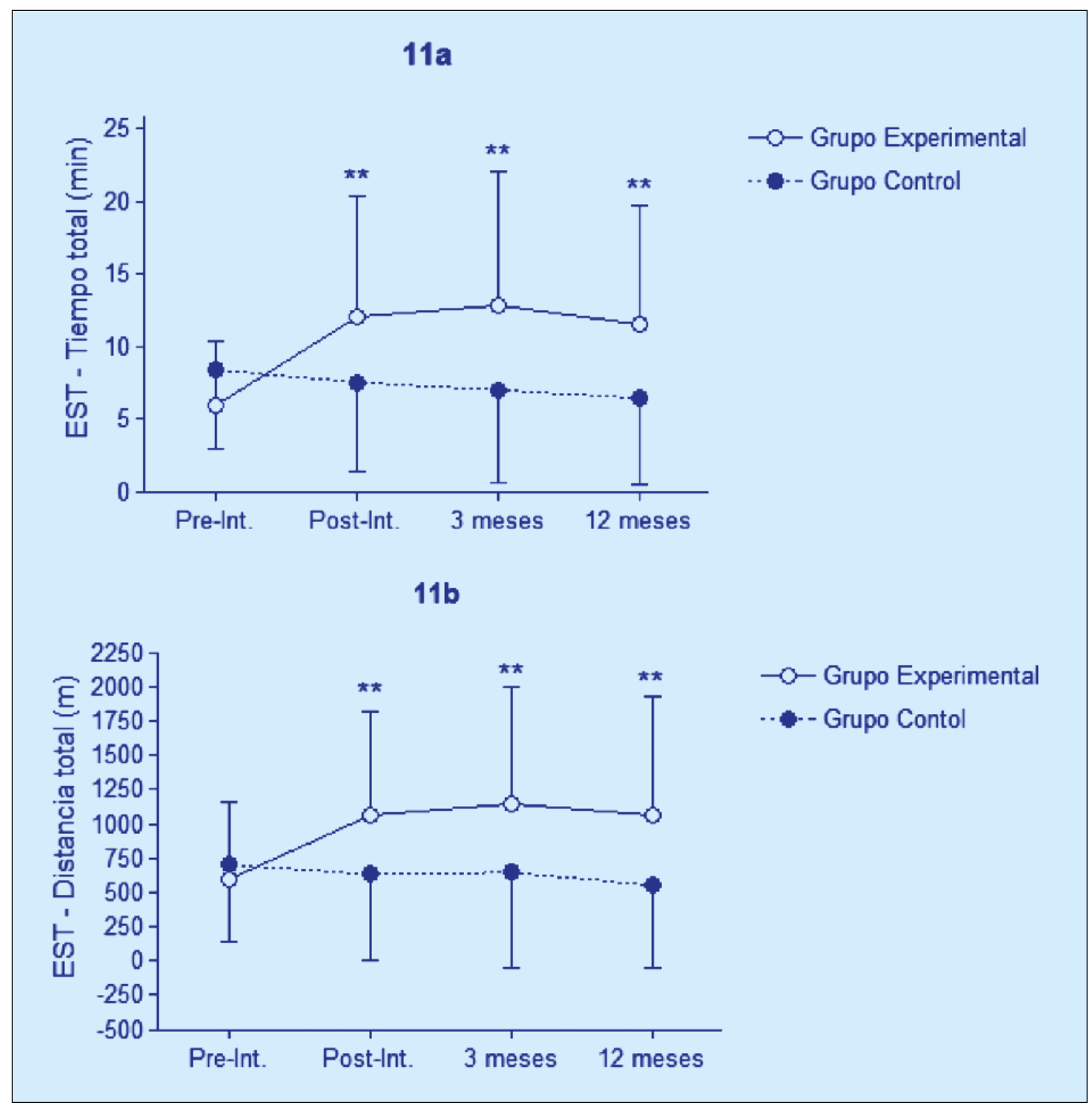

Se representa la media y la desviación estándar del tiempo (11a) y la distancia (11b) alcanzados en la Prueba de Lanzadera a Carga Constante (EST) para cada grupo de estudio, en los distintos períodos de registro: Previo a la intervención (Pre-Int.), inmediatamente tras la finalización de los programas (Post-Int.), a los 3 meses (3 meses) y a los 12 meses (12 meses) del registro post-intervención.

$(* *), p<0,01$ para la comparación intergrupo post-intervención vs pre-intervención y a los 3 y 12 meses vs pre-intervención. 
meses en relación al registro pre-intervención ((-6,7 $\pm 5,2$ puntos; $\left.95 \% \mathrm{IC} .(-9,5 ;-3,8) ; p=0,000^{f}\right)$ y $\left(-6,8 \pm 7,8\right.$ puntos; $95 \%$ IC. $\left.(-10,8 ;-2,8) ; p=0,002^{f}\right)$, respectivamente) y del $\approx 13 \%$ y $\approx 21 \%$ a los 12 meses $\left(\left(-5,4 \pm 5\right.\right.$ puntos; $95 \%$ IC. $\left.(-8 ; 2,8) ; p=0,000^{b}\right)$ y $(-6,6 \pm 6,8$ puntos; $95 \%$ IC. $(-10,2 ;-3,1)$; $\left.\mathrm{p}=0,001^{\mathrm{f}}\right)$, respectivamente). Por su parte, en el grupo control continuaron aumentando estas puntuaciones significativamente, tanto a los 3 meses $((2,3 \pm 3,8$ puntos; $95 \% \mathrm{IC} .(0,3 ; 4,3)$; $\left.\mathrm{p}=0,028^{f}\right)$ en la puntuación total y $\left(4 \pm 5,8\right.$ puntos; $95 \% \mathrm{IC}$. $\left.(0,8 ; 7) ; \mathrm{p}=0,016^{f}\right)$ en la dimensión de impacto) como a los 12 meses ( $\left(3,2 \pm 4,1\right.$ puntos; $95 \%$ IC. $\left.(1 ; 5,4) ; p=0,006^{f}\right)$ y $(5,7 \pm 6$ puntos; $95 \%$ IC. $\left.(2,5,8) ; p=0,002^{f}\right)$, respectivamente). Las diferencias intergrupo fueron estadísticamente significativas, mientras que no se observaron cambios relevantes en las dimensiones de actividad y síntomas.

Gráfica 12. Efecto de los programas sobre el nivel de actividad física.

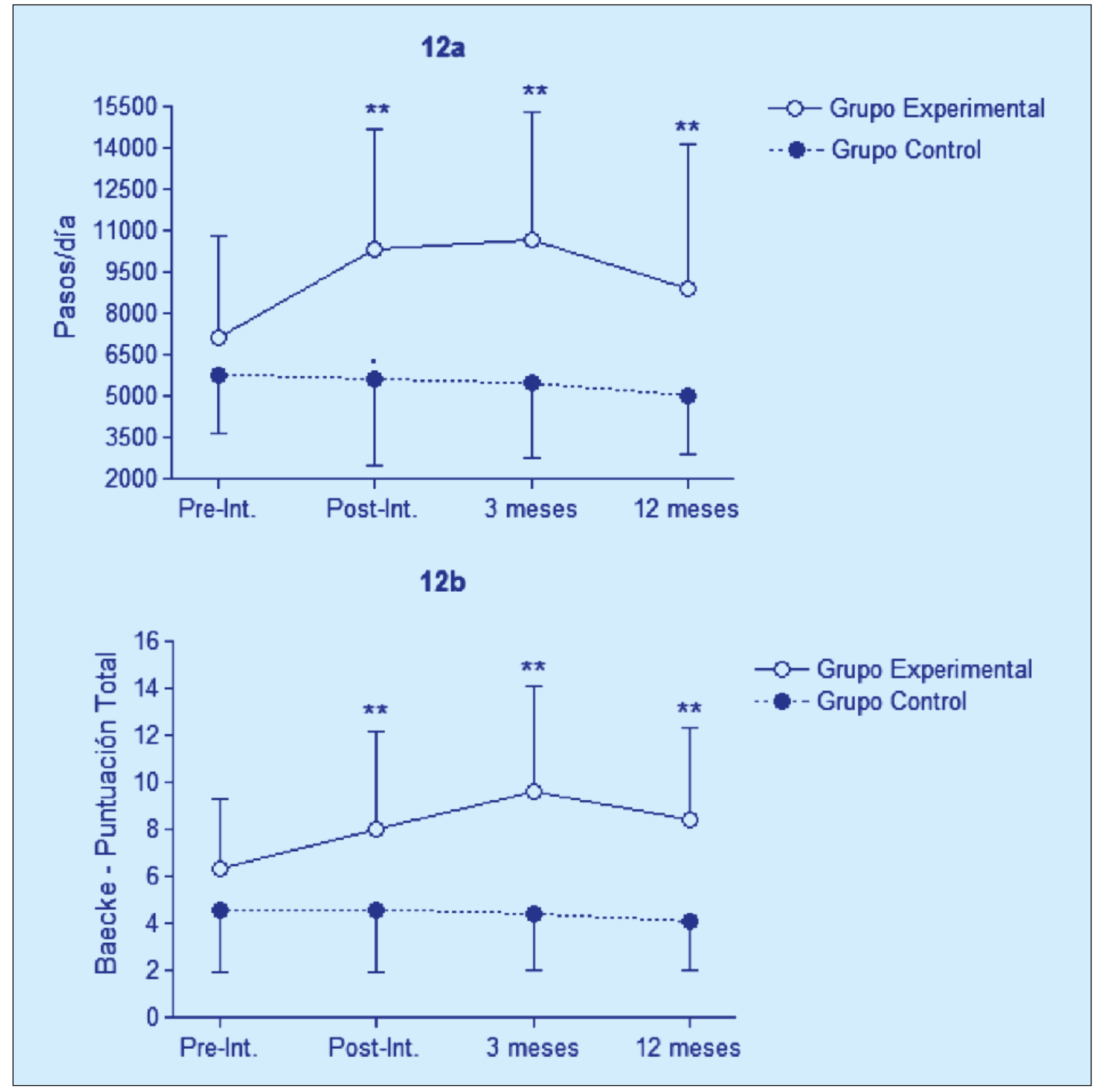

Se representa la media y la desviación estándar del número de pasos al día (12a) y la puntuación total del Cuestionario Baecke Modificado (12b) para cada grupo de estudio, en los distintos períodos de registro: Previo a la intervención (Pre-Int.), inmediatamente tras la finalización de los programas (Post-Int.), a los 3 meses (3 meses) y a los 12 meses (12 meses) del registro post-intervención.

$\left.{ }^{* *}\right), p<0,01$ para la comparación intergrupo post-intervención vs pre-intervención y a los 3 y 12 meses vs pre-intervención. 
La disnea basal se mantuvo en valores muy similares al registro post-intervención en ambos grupos. Frente a los valores pre-intervención, la puntuación en la Escala mMRC no presentó cambios significativos ni a los 3 ni a los 12 meses ni en el grupo experimental ni en el grupo control.

En cuanto al número de exacerbaciones sufridas en los 12 meses anteriores al inicio del estudio, fue similar en ambos grupos (rango, 0-5) y se redujo ligeramente en los 12 meses de seguimiento (rango, 0-2 en el grupo experimental y 0-4, en el control). Esta reducción no fue significativa ni en el grupo experimental ni en el control.

En las Gráficas 13, 14 y 15 se representa la evolución a lo largo del estudio, de la puntuación total del SGRQ, la disnea basal según la Escala mMRC y el número de exacerbaciones, para cada grupo.

\section{Análisis de covarianza}

Se aplicó el análisis de covarianza en las variables en las que se obtuvieron diferencias significativas entre los grupos (tolerancia al ejercicio, pasos/día, puntuación en el Cuestionario de Actividad Física Baecke Modificado y en el de calidad de vida). De éstas, el modelo de regresión cumplía las condiciones de aplicabilidad en los pasos/día y en el cuestionario de calidad de vida, obteniéndose resultados significativos para ambas variables en los tres momentos de medición. En la Tabla 14 se muestra el efecto de la intervención no ajustado y ajustado según valores basales para las variables anteriores.

\subsection{Análisis de sensibilidad}

Se llevó a cabo un análisis por intención de tratar en las variables distancia y tiempo alcanzados en el EST, pasos/día y la puntuación total del Cuestionario Baecke Modificado. Los datos perdidos se completaron con los valores basales de cada sujeto excluido. En el análisis

Gráfica 13. Efecto de los programas sobre la calidad de vida.

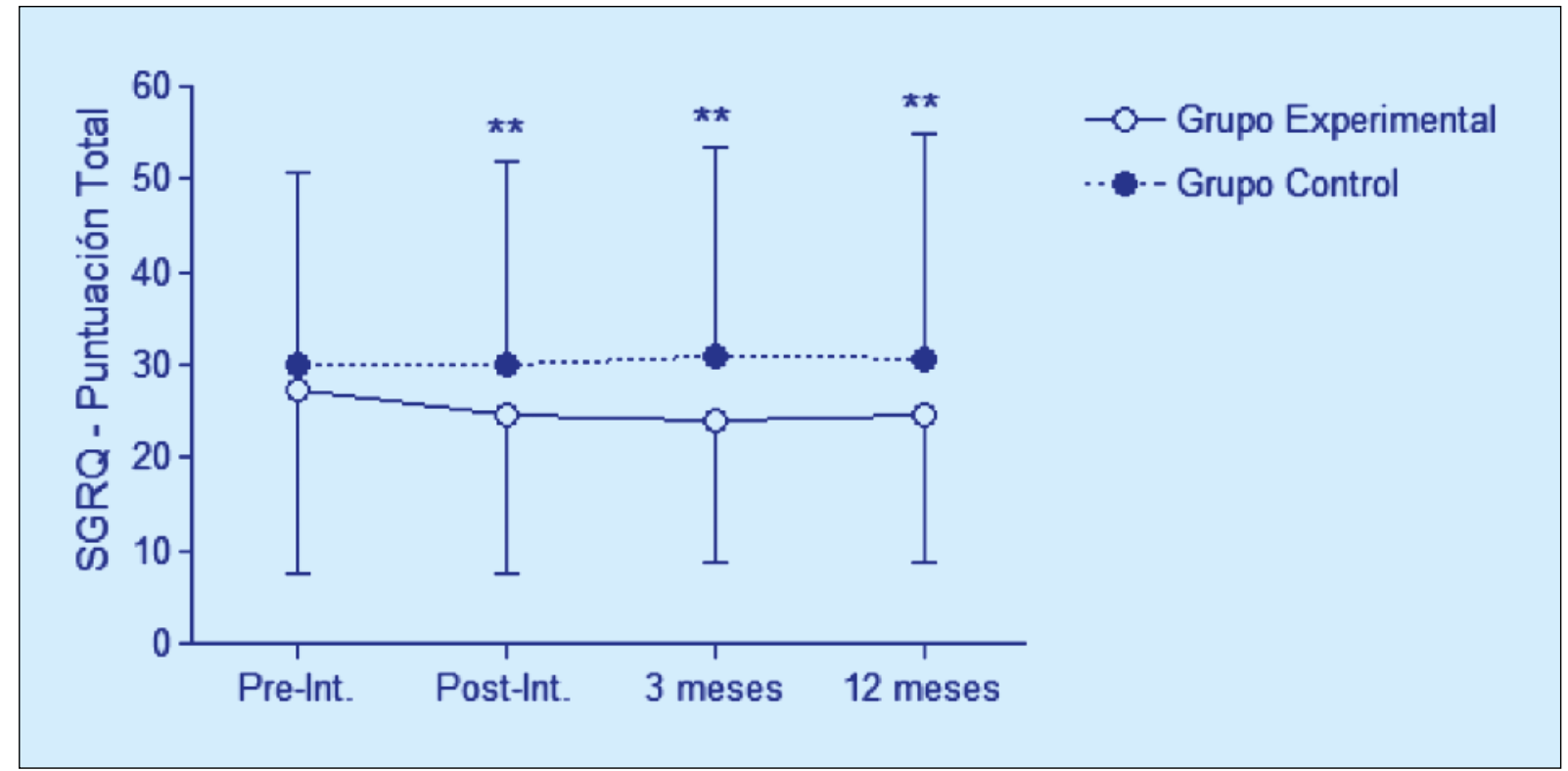

Se representa la media y la desviación estándar de la puntuación total obtenida en el Cuestionario St. George's (SGRQ) para cada grupo de estudio, en los distintos períodos de registro: Previo a la intervención (Pre-Int.), inmediatamente tras la finalización de los programas (Post-Int.), a los 3 meses (3 meses) y a los 12 meses (12 meses) del registro post-intervención.

$\left.{ }^{* *}\right), p<0,01$ para la comparación intergrupo post-intervención vs pre-intervención y a los 3 y 12 meses vs pre-intervención. 
de sensibilidad se mantuvieron unos resultados similares a los obtenidos en el análisis por protocolo (Tabla 15).

Gráfica 14. Efecto de los programas sobre la disnea basal.

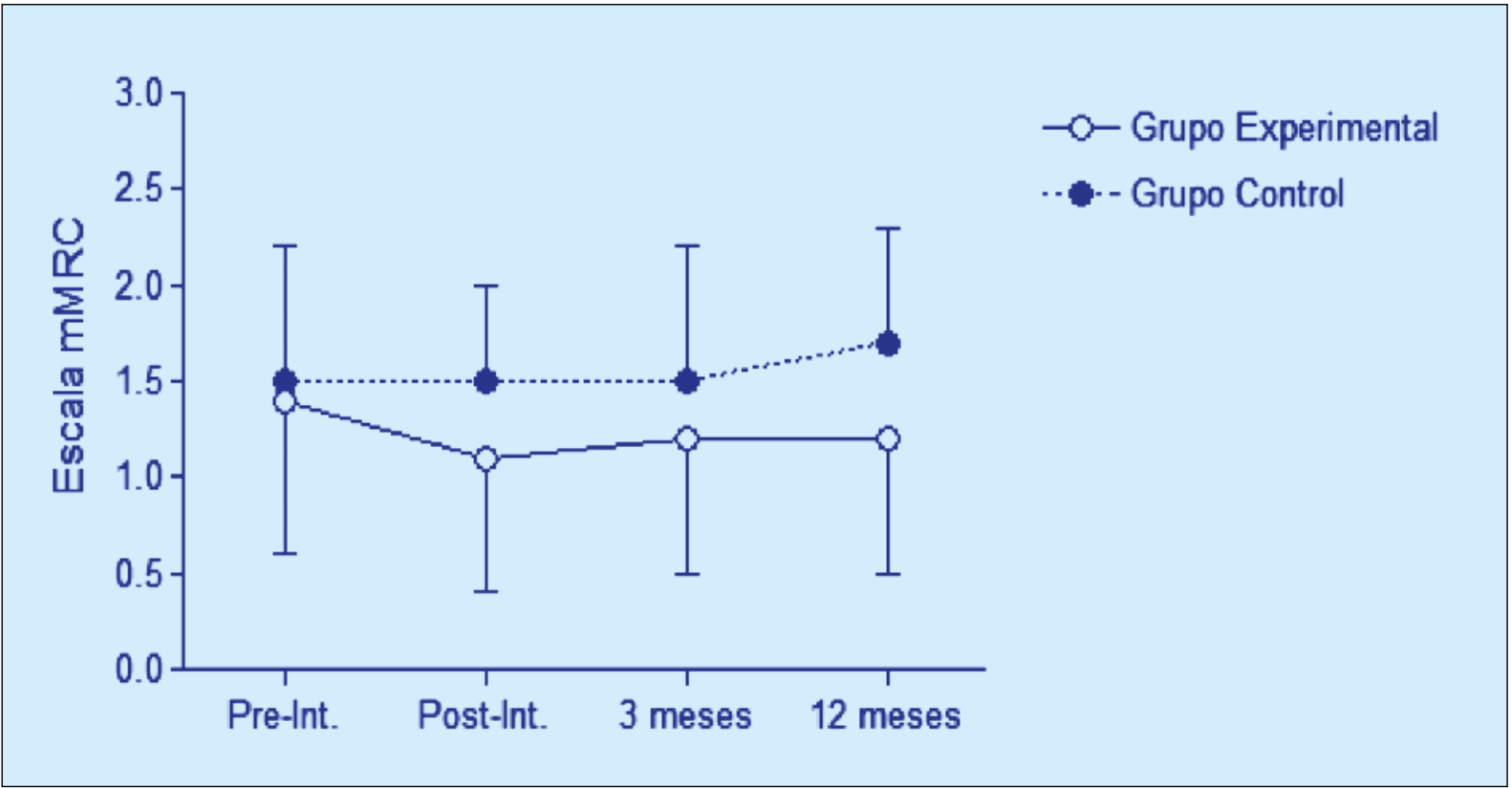

Se representa la media y la desviación estándar de la puntuación obtenida en la Escala Modificada del Medical Research Council (mMRC) para ambos grupos de estudio, en los distintos períodos de registro: Previo a la intervención (Pre-Int.), inmediatamente tras la finalización de los programas (Post-Int.), a los 3 meses (3 meses) y a los 12 meses (12 meses) del registro post-intervención.

No se encontraron diferencias significativas en la comparación intergrupo post-intervención vs preintervención, ni a los 3 y 12 meses vs pre-intervención.

\section{Gráfica 15. Efecto de los programas sobre las exacerbaciones.}

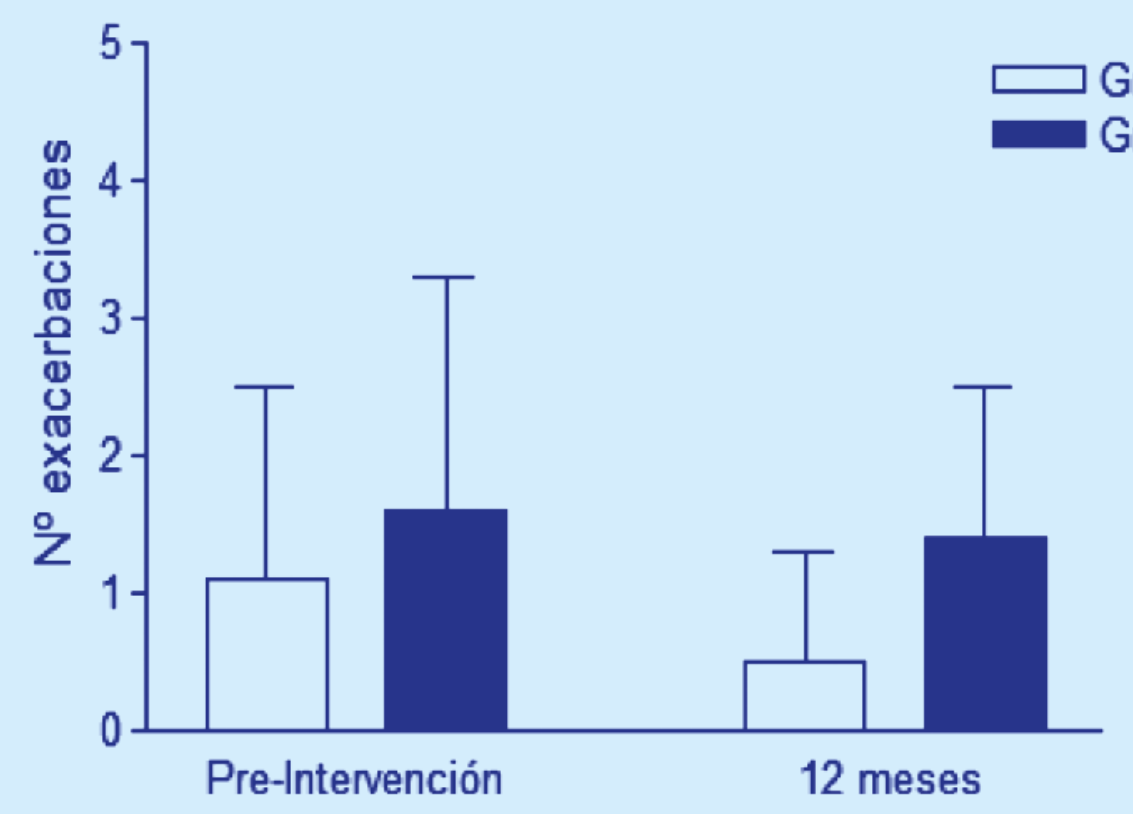

Se representa la media y la desviación estándar del número de exacerbaciones sufridas en los 12 meses de seguimiento frente a los 12 meses anteriores al comienzo de la intervención (Pre-Intervención) en cada grupo de estudio.

No se encontraron diferencias significativas en la comparación intergrupo a los 12 meses vs pre-intervención. 

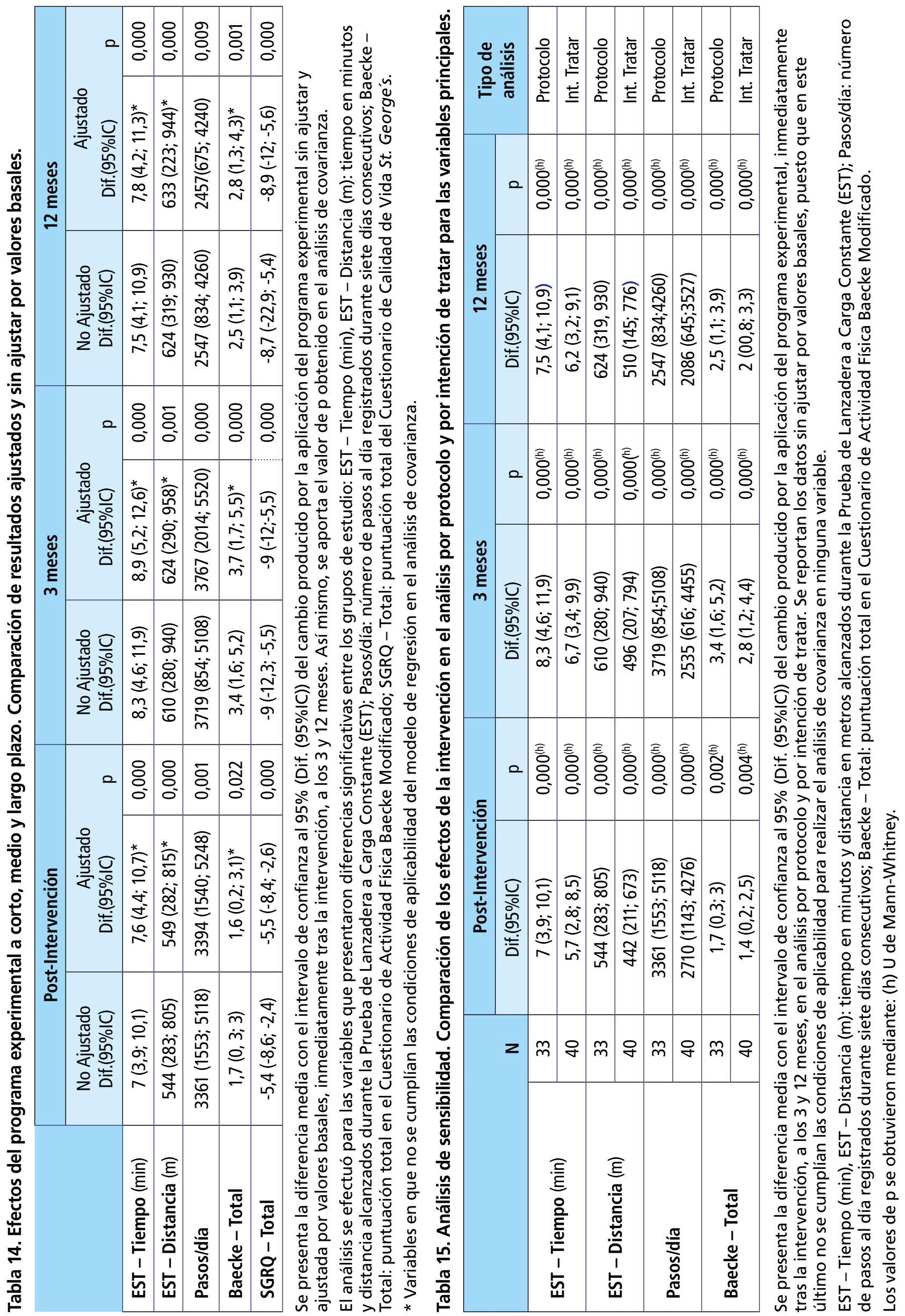


\section{Diferencias en los resultados según distintos factores ${ }^{1}$}

Se evaluó si existían diferencias en los resultados, segmentando cada grupo de estudio por el sexo, la edad (hasta 60 años o superior a 60 años), la severidad de la EPOC (Grados I-II y Grupos III-IV de la clasificación GOLD(43)), el uso o no de OCD y el período en que se realizó la intervención (otoño-invierno o primavera-verano). Sólo se encontraron diferencias relevantes y significativas para el tiempo alcanzado en el EST en el grupo experimental segmentado según la severidad de la EPOC (Tabla 16).

En este caso, tras la intervención apenas se hallaron diferencias entre los dos subgrupos en relación al registro pre-intervención $((6,1 \pm 6,9 \mathrm{~min} ; 95 \% \mathrm{IC}$. $(-1,2 ; 13,5) ; \mathrm{p}=0,028)$ en el grupo con GOLD I-II y $\left(6 \pm 5,4 \mathrm{~min} ; 95 \% \mathrm{IC}\right.$. $\left.(2,5 ; 9,7) ; p=0,003^{\mathrm{e}}\right)$ para el grupo con GOLD III-IIV). Sin embargo, a los 3 meses vs pre-intervención se observó que los sujetos con GOLD I-II presentaban un mayor incremento en la variable $\left(7,6 \pm 8,4 \mathrm{~min} ; 95 \% \mathrm{IC}\right.$. $\left.(-0,9 ; 15,9) ; p=0,028^{\mathrm{e}}\right)$ que los sujetos con GOLD III-IV $\left(6,5 \pm 6,3\right.$ min; $95 \%$ IC. $\left.(2,3 ; 9,7) ; p=0,03^{e}\right)$. A los 12 meses vs pre-intervención se invirtió la tendencia y fue el grupo de sujetos más severos el que alcanzó mejores resultados $\left(5,8 \pm 6,2 \mathrm{~min} ; 95 \%(1,7 ; 10) ; p=0,005^{e}\right)$ frente a $\left(4,9 \pm 7,1 \mathrm{~min} ; 95 \% \mathrm{IC} .(-2,5 ; 12,4) ; p=0,043^{e}\right)$.

\section{Asociación entre la modificación del nivel de actividad física y la tolerancia al esfuerzo, la calidad de vida, la disnea basal y las exacerbaciones}

\section{A los 3 meses tras la intervención}

En el total de sujetos de la muestra se produjo un incremento de $1.290 \pm 3.515$ pasos/día en re-

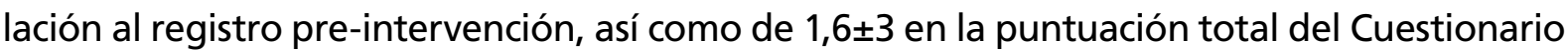
de Actividad Física Baecke Modificado. Por su parte, el tiempo total alcanzado y la distancia recorrida en el EST aumentaron $(2,9 \pm 6,5 \mathrm{~min}$. y $256,9 \pm 552,5 \mathrm{~m}$, respectivamente), la puntuación total del SGRQ se redujo $(-2,3 \pm 6,5)$, mientras que la disnea basal (Escala mMRC) apenas se modificó $(-0,1 \pm 0,4)$.

En el análisis de asociación controlando por factores confusores, se encontró una correlación significativa entre el incremento en el nivel de actividad física y la mejora en los resultados del EST, así como en la calidad de vida (reducción en la puntuación total del SGRQ). Por otro lado, en el caso de la disnea basal, la asociación no fue significativa (Tabla 17)

\section{A los 12 meses tras la intervención}

Al final del período de seguimiento, en el total de sujetos de la muestra se observó un incremento de $532 \pm 2.702$ pasos/día en relación al registro pre-intervención, así como de $0,8 \pm 2,4$ en la puntuación total del Cuestionario Baecke Modificado. Por su parte, el tiempo total y la

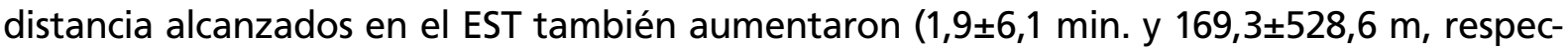
tivamente), la puntuación total del SGRQ se redujo $(-1,2 \pm 6,3)$, mientras que la disnea basal (Escala mMRC) no se modificó $(0 \pm 0,5)$.

En el análisis de la asociación entre las variables, se observó el mismo comportamiento descrito para el registro de 3 meses post-intervención (Tabla 17).

En cuanto al número de exacerbaciones sufridas durante los 12 meses de seguimiento por la totalidad de los sujetos de estudio, fue de $0,9 \pm 1,1$. No se encontró asociación significativa entre esta variable y la variación en el número de pasos/día o en la puntuación total del Cuestionario Baecke (Tabla 17).

En las Gráficas 16 y 17 se representa la asociación entre el incremento en el nivel de actividad física (evaluado mediante pasos/día y puntuación total del Cuestionario Baecke) a los 12 meses tras la intervención, con la tolerancia al ejercicio y la calidad de vida. 

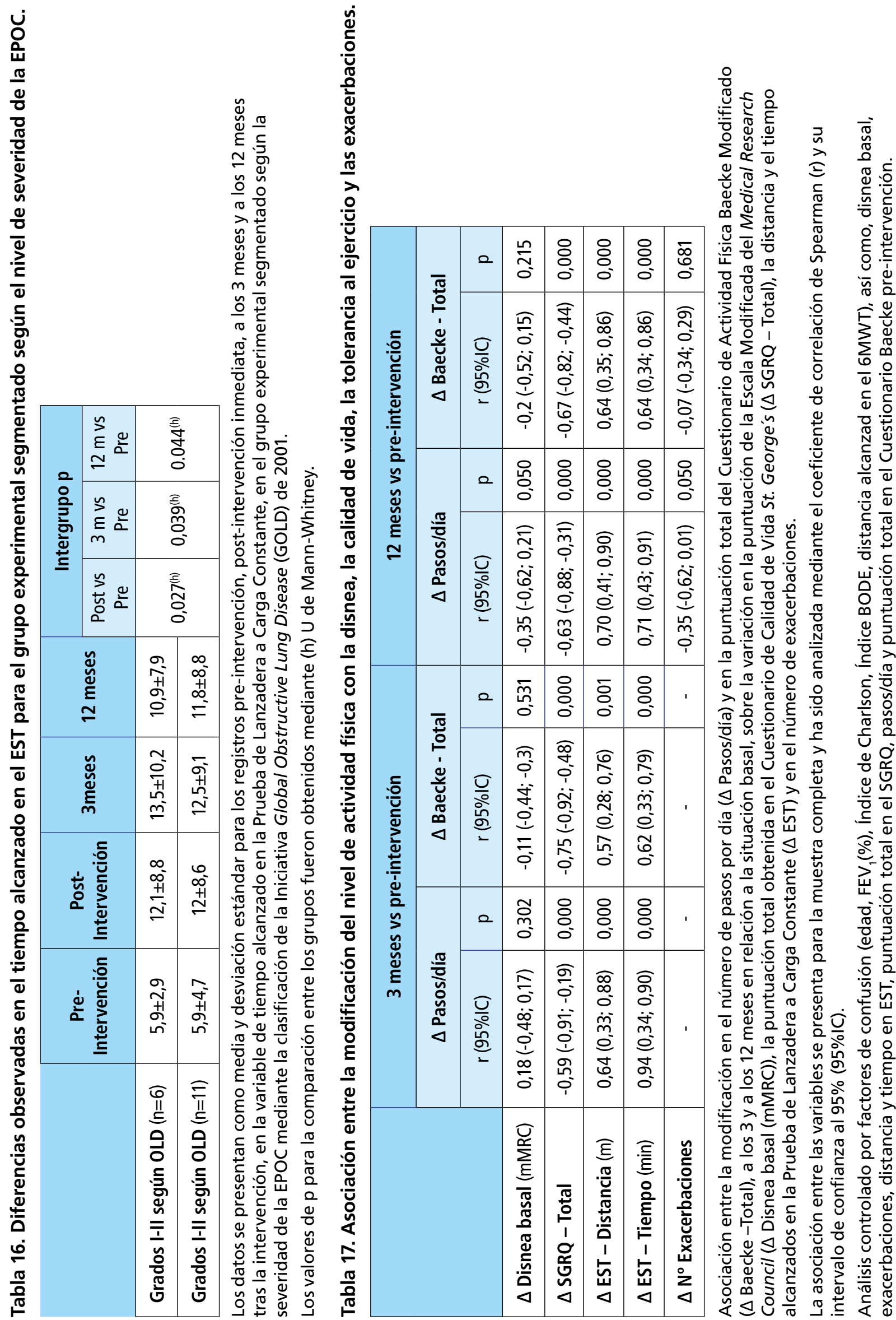
Gráfica 16. Asociación entre el incremento en el nivel de actividad física y la tolerancia al ejercicio.
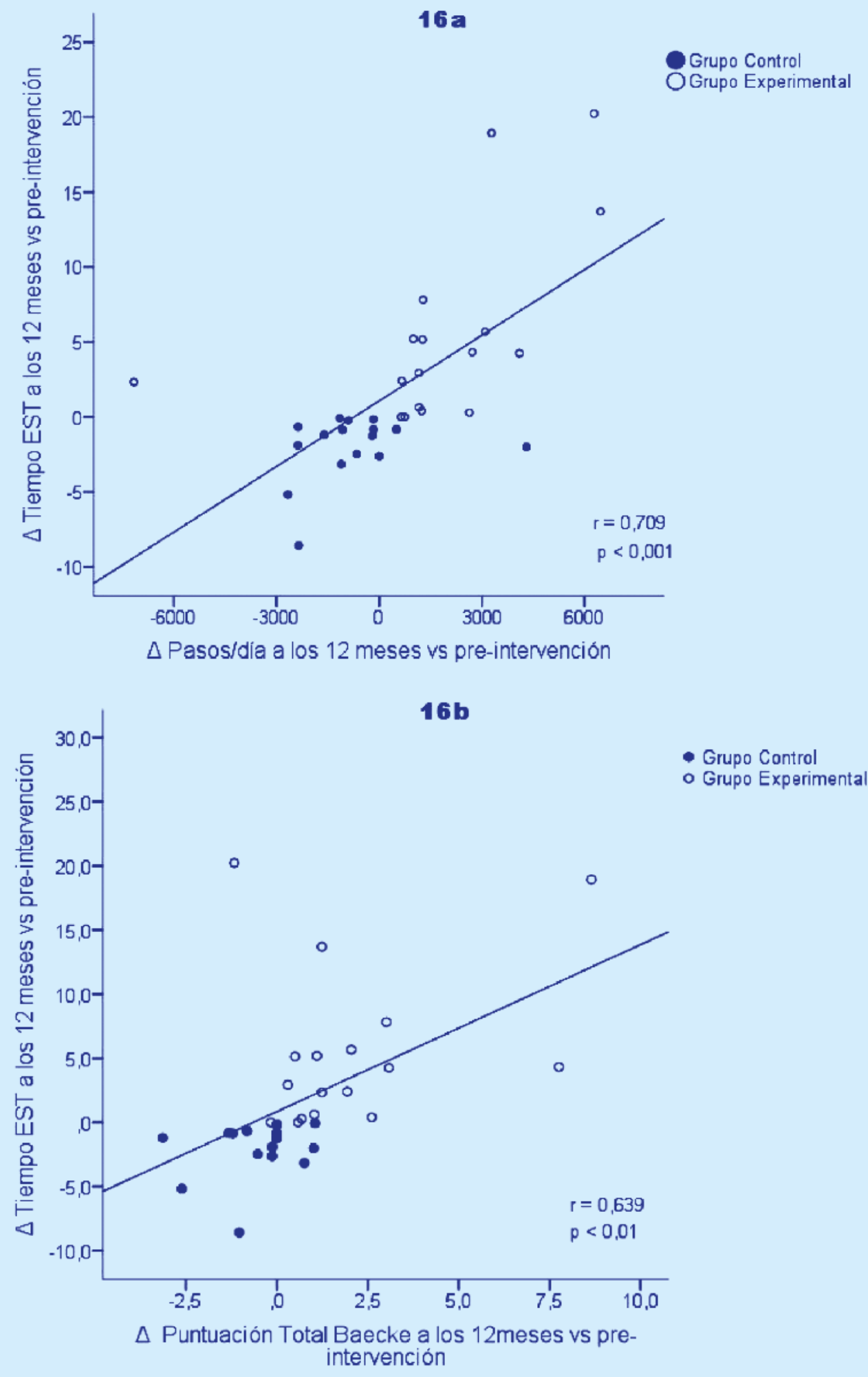

La línea representa la relación existente entre la variación en el número de pasos diarios (16a) y en la puntuación total del Cuestionario de Actividad Física Baecke Modificado (16b) a los 12 meses de finalizar la intervención vs previo a la intervención, con el cambio en el tiempo total alcanzado en la Prueba de Lanzadera a Carga Constante (EST). 
Gráfica 17. Asociación entre el incremento en el nivel de actividad física y la calidad de vida.
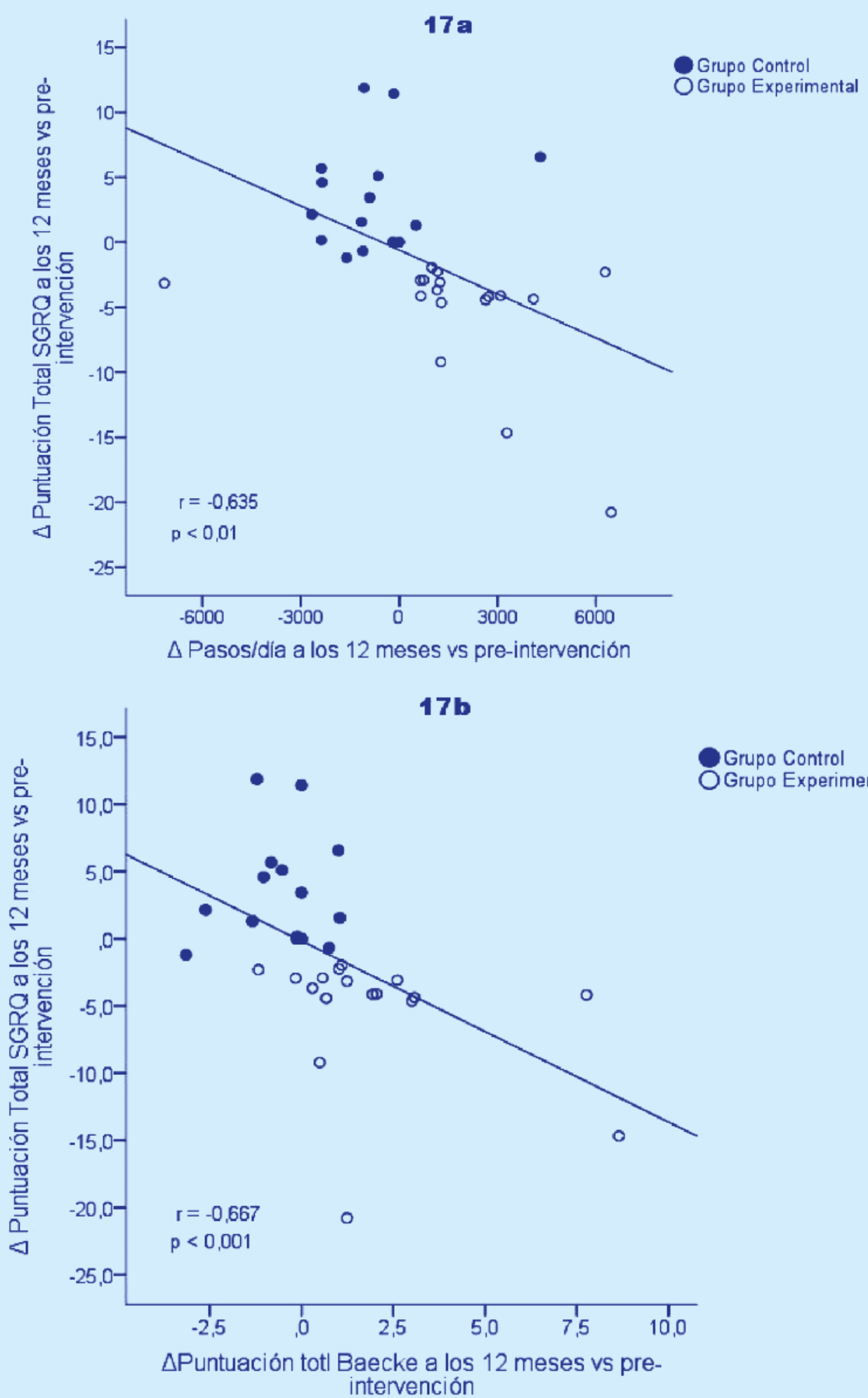

La línea representa la relación existente entre la variación en el número de pasos diarios (17a) y en la puntuación total del Cuestionario de Actividad Física Baecke Modificado (17b) a los 12 meses de finalizar la intervención vs previo a la intervención, con el cambio en la puntuación total del Cuestionario de Calidad de Vida St. George's (SGRQ).

(1) Las pruebas utilizadas para el cálculo del valor de $p$ en la comparación itnragupo de las variables descritas en los apartados 2. y 3, se indican codificadas dentro de los paréntesis de resultados, siendo: (e),T para datos pareados y (f), Wilcoxon. 


\section{PARTE IV \\ DISCUSIÓN Y CONCLUSIONES}

\section{DISCUSIÓN}

\section{Efectos de la aplicación de programas ambulatorios de Rehabilitación Respiratoria: Su duración y los factores influyentes}

En el Estudio 1 de esta tesis se demostró que las tres modalidades de programas ambulatorios de RR aplicados en la Escuela Universitaria de Fisioterapia de la ONCE, conseguían aumentar la distancia de la prueba de 6MWT y reducir la puntuación en la escala de disnea mMRC. Además, en aquellos que entrenaron específicamente los músculos respiratorios, también incrementaron sus presiones respiratorias máximas. Sin embargo, sin la incorporación de estrategias de mantenimiento, estos resultados se perdieron durante el período de seguimiento, sin diferencias entre los distintos programas.

\section{Efectos tras la aplicación de los programas}

El incremento en la distancia del 6MWT en los tres programas superó la MCID de 30 o 35 metros (249,273-275)), lo que significa que fue percibido como una mejoría en la capacidad de ejercicio por parte de los pacientes. Por otro lado, la reducción en la Escala mMRC indica que los pacientes experimentaron una disminución en su percepción de disnea habitual. Estos resultados ponen de manifiesto la efectividad de la RR ambulatoria en cuanto a la mejora en la capacidad ejercicio y la disnea basal, manteniendo una coherencia con las revisiones publicadas por Lacasse y col. (73) y Puhan y col. (74), así como con las conclusiones de las principales guías de práctica clínica de manejo de estos pacientes $(1,2,34,48,49,72,81)$. Sin embargo, no se produjo una reducción en las exacerbaciones tras la RR, como sí se observó en investigaciones precedentes $(73,292)$. Este hecho se puede asociar probablemente a que el tamaño muestral no fue suficiente para obtener diferencias significativas o bien, porque el sistema de registro de las exacerbaciones no fue lo bastante fiable, ya que se basó en la información autorreportada por los pacientes.

Estos resultados no fueron diferentes según el tipo de programa aplicado. Aunque parece que la incorporación de entrenamiento de fuerza de los miembros superiores e inferiores, así como específico de los músculos respiratorios, mejoró los resultados en mayor medida que el entrenamiento aeróbico aislado, estas diferencias no alcanzaron significación estadística, probablemente debido al bajo tamaño muestral.

Por otro lado, en el grupo que recibió entrenamiento específico de los músculos respiratorios, se observó una mejoría en las presiones respiratorias máximas de en torno a un $20 \%$. Esto viene a confirmar las conclusiones reportadas en varias revisiones sobre el incremento en la fuerza muscular respiratoria tras el IMT (293-295) o combinado IMT + EM) (296). Según Gosselink y col. (293), el IMT asociado a entrenamiento aeróbico general en pacientes con debilidad inspiratoria, aumenta los beneficios sobre la capacidad de ejercicio y la disnea. En el programa que analizamos se añadió IMT y EMT en los pacientes que presentaban debilidad en los músculos respiratorios, lo que puede justificar que se produjeran mejores resultados en cuanto al 6MWT y la disnea basal en comparación con los pacientes que sólo recibieron entrenamiento aeróbico, a pesar de que las diferencias entre grupos no fueran significativas. 
Otra posible explicación de la no significación de estos resultados puede achacarse a que en comparación con estudios anteriores $(296,297)$, la mejoría en PIM y PEM fue menor en nuestro grupo. Las características de los sujetos no justifican las diferencias con nuestro programa, ya que en todos los casos se analizaron fundamentalmente pacientes EPOC moderados-severos. Tampoco, los resultados pueden ser atribuidos a la intensidad del entrenamiento, que superaba el $50 \%$ de la PIM-PEM en ambos programas. Sin embargo, consideramos que quizás pudo influir la duración del entrenamiento, ya que en nuestro caso fue de ocho semanas, mientras que en los anteriormente referidos se realizaron al menos 12 semanas.

Por último, al evaluar qué factores pudieron influir en los resultados de los programas sobre la tolerancia al ejercicio, sólo se hallaron diferencias significativas al segmentar la muestra según el número de exacerbaciones en los 12 meses previos a la inclusión en los programas ( 0 vs $1-2$ exacerbaciones). Tanto en los pacientes que no habían presentado exacerbaciones previas como los que reportaron 1-2 cuadros de reagudización, la distancia del 6MWT mejoró por encima de la MCID, pero en mayor medida en los individuos sin exacerbaciones previas. Los sujetos no presentaban diferencias en cuanto a severidad de la enfermedad, edad u otros factores que pudieran afectar a los resultados del entrenamiento, por lo que pensamos que éstos se pueden atribuir a factores individuales y/o psicológicos no controlados (una mejor respuesta al ejercicio en los sujetos que no "temían" sufrir una reagudización porque no tenían recuerdos recientes de episodios similares). Por otro lado, no se tuvo en cuenta la duración de las exacerbaciones, si bien, ésta puede ser un condicionante más importante en la capacidad de ejercicio que su frecuencia (123).

\section{Duración de los efectos}

La pérdida progresiva de los efectos alcanzados tras la RR que se observa en la muestra del Estudio 1 es coherente con la evidencia actual, que afirma que los beneficios de la RR se pierden entre los 6 y los 12 meses (112). A pesar de que algunos estudios han reportado que este declive está relacionado con la evolución de la enfermedad (111), esto no parece haber influido en los sujetos de nuestro estudio, ya que no se produjeron cambios significativos en la función pulmonar durante el seguimiento. Del mismo modo, el número de exacerbaciones en este período no varió en relación a los 12 meses previos, por lo que contrario a lo que indican algunas investigaciones $(121,123)$, las exacerbaciones no fueron un factor influyente en la pérdida de la capacidad de ejercicio en nuestro caso.

La ausencia de diferencias significativas en estos hallazgos entre los tres programas aplicados confirma las conclusiones publicadas en la revisión de Ochman y col. (114), en la que afirman que la pérdida de los efectos tras la RR es independiente del tipo de programa utilizado siempre que no se incorporen estrategias para el cambio comportamental de los pacientes. No obstante, en nuestra muestra parece que los individuos que además de realizar entrenamiento aeróbico, llevaron a cabo entrenamiento de fuerza de los miembros y específico de los músculos respiratorios, tuvieron una disminución menor en la distancia del 6MWT. Estos resultados podrían atribuirse al efecto sumatorio que se produce cuando se combinan diferentes modalidades de ejercicio. Sería necesario analizar a un mayor número de sujetos para determinar si estas diferencias pueden ser significativas.

Por otro lado, en el grupo que entrenaron los músculos respiratorios también se observó una pérdida casi total del incremento alcanzado en las presiones respiratorias máximas tras el entrenamiento. Esto es coherente con el estudio de Weiner y col. (297), en el que hallaron que tras un programa de IMT de 12 semanas al $60 \%$ de la PIM, el declive comenzaba a partir del 
tercer mes de la finalización del programa y era completo a los 12 meses. Por su parte, Güell y col. (107), aplicando un IMT al $40 \%$ de la PIM, tres días por semana, asociado a un programa ambulatorio y a uno domiciliario, no encontraron una reducción significativa de los beneficios en los seis meses siguientes, pero sí una tendencia decreciente, lo que hace pensar que si el período de seguimiento hubiera sido más largo también se hubieran alcanzado pérdidas significativas. En cuanto al EMT, no hemos encontrado estudios que evalúen específicamente la duración de sus efectos a medio y largo plazo.

Por último, al evaluar la influencia de determinados factores en la duración de los efectos sobre la distancia alcanzada en el 6MWT, sólo se hallaron diferencias significativas entre los pacientes que habían mejorado en esta variable por encima de la MCID y los que no la superaron. En el primer grupo, al final del período de seguimiento aún mantenían un incremento de alrededor del 5\% sobre sus niveles basales. Así mismo, el hallazgo de una correlación significativa e inversa aunque moderada, entre estas variables, viene a confirmar lo anterior. Es decir, en los sujetos del estudio, cuanto mayor fue la mejoría en la capacidad de ejercicio, más lento fue el deterioro subsiguiente. Mediante un modelo de regresión lineal se ha obtenido la ecuación de predicción, que aporta una estimación de la pérdida en la distancia del 6MWT a partir de la ganancia obtenida tras el entrenamiento. El tamaño muestral no permite extrapolar el modelo a la población diana, sin embargo, el interés de nuestra investigación radica en que es el primer estudio que analiza y reporta resultados acerca de la asociación entre efectos post-entrenamiento y mantenimiento de los mismos.

Por tanto y en resumen, podemos afirmar que en la muestra analizada, los efectos tras la RR se perdieron prácticamente en su totalidad alrededor de 12 meses tras la finalización de los programas. Esto no se vio claramente influenciado ni por las características de los programas ni por factores adicionales como el sexo, la edad, el IMC, si habían sido fumadores o no habían fumado nunca, si necesitaban OCD o por la severidad y evolución de la enfermedad, si bien, sería necesaria una muestra mayor para poder establecer afirmaciones concluyentes. Parece que el único factor relevante fue el hecho de optimizar los resultados sobre la capacidad de ejercicio tras los programas de entrenamiento. Esto pone de manifiesto la importancia de diseñar programas de RR eficaces, que consigan cambios en la tolerancia al ejercicio de los pacientes, clínicamente significativos.

\section{Eficacia del entrenamiento domiciliario en los pacientes EPOC}

En el Estudio 2 de esta tesis se ha demostrado que el programa de RR autoadministrado aplicado mejoró significativamente la distancia y el tiempo alcanzados en el EST, así como la calidad de vida evaluada mediante el Cuestionario SGRQ, manteniéndose estos resultados a medio y largo plazo (3 y 12 meses tras la intervención). Por el contrario, no se obtuvieron cambios en la puntuación de la escala de disnea mMRC ni en el número de exacerbaciones.

\section{Efectos a corto plazo}

En nuestro estudio, tanto la distancia como el tiempo alcanzados en el EST se duplicaron en relación al valor basal y superaron ampliamente la MCID $(277,281)$. Estos resultados significan que los cambios obtenidos fueron percibidos como una mejoría clínica por los pacientes. No se han encontrado investigaciones anteriores que evalúen la capacidad de ejercicio mediante el EST tras un PRR domiciliario similar al nuestro. Sin embargo, varios autores utilizaron el IST $(102,299)$ o el 6MWT $(99,103,105)$. 
Liu y col. (102) reportaron un incremento significativo en la distancia alcanzada en el IST tras la aplicación de un programa de marcha de 12 semanas, en el que los pacientes debían caminar un mínimo de cuatro días por semana a una velocidad correspondiente al $80 \%$ del $\mathrm{VO}_{2}$ max. El entrenamiento se realizaba siguiendo el ritmo de un programa musical que llevaban instalado en el teléfono móvil. Por el contrario, en el estudio de Dias y col. (298) no se alcanzaron cambios significativos en esta prueba, tras un programa también de caminata a alta intensidad $\left(85 \%\right.$ del $\mathrm{VO}_{2}$ max) durante 12 semanas, tres días por semana durante 30-40 minutos al día. La gran diferencia entre ambos programas fue el autocontrol de la intensidad del entrenamiento, ya que en este último los pacientes eran adiestrados previamente en la velocidad de caminata, pero no contaban con ningún sistema de control específico durante las mismas, por lo que es probable que no se entrenara a la intensidad pautada durante la mayor parte del tiempo de ejercicio (298). En este sentido, el empleo del podómetro en nuestro estudio, permite garantizar que los sujetos entrenaban al nivel previsto.

Por su parte, van Wetering y col. (99) y Pradella y col. (103), utilizando como pauta de intensidad, la frecuencia cardiaca (60-80\% de la FC teórica máxima), entrenando mediante marcha durante 30 minutos, tres días por semana, 12 u 8 semanas, respectivamente, obtuvieron una mejoría superior a la MCID del 6MWT. Así mismo, Pomidori y col. (105) también alcanzaron un incremento clínica y estadísticamente significativo en la distancia del 6MWT tras un PRR domiciliario de larga duración (seis meses). Éste se basó en caminar a una intensidad moderada ( $50 \%$ del $\mathrm{VO}_{2}$ max) durante 20-30 minutos, cuatro días a la semana. La velocidad de caminata se regulaba mediante un metrómetro o a través del objetivo de completar una distancia en un tiempo determinado por los investigadores.

Los resultados en el EST son comparables a los obtenidos en algunos programas ambulatorios de alta intensidad $(130,131)$. Incluso, el efecto de la intervención obtenido en nuestro estudio fue de mayor magnitud que en el estudio de Wootton y col. (299). En éste se aplicó un programa ambulatorio de 20-24 sesiones, basado exclusivamente en marcha a una velocidad correspondiente al $80 \%$ de la alcanzada en un 6MWT. Consideramos que la clave podría estar en la intensidad del entrenamiento, ya que en el programa aplicado por Wootton ésta era ligeramente inferior a nuestra intervención.

Del mismo modo, en el programa COPE-Active, llevado a cabo en Dinamarca, los cambios en el EST no fueron tan relevantes. Se trataba de un programa basado en la comunidad, de larga duración, dirigido además a modificar los hábitos de actividad de los pacientes. Los sujetos incluidos acudían a sesiones presenciales de entrenamiento en cicloergómetro, dos días a la semana, durante seis meses, completando con una tercera sesión domiciliaria mediante caminata, todo ello al $85 \%$ del $\mathrm{VO}_{2}$ max. Effing y col. (132) analizaron este programa y hallaron un incremento significativo pero modesto, en los sujetos del grupo experimental (106 m). Posteriormente, Zwerink y col. (218) evaluaron este mismo protocolo añadiendo tras los primeros seis meses, una segunda fase de cinco meses de duración, con las mismas características pero reduciendo las sesiones presenciales a una semanal. Al final de estos dos períodos, el incremento en la distancia del EST seguía siendo moderado (146 m). Las características de los sujetos no justifican las diferencias con nuestros hallazgos, ya que presentaban un FEV (\%) similar a nuestra muestra- Es probable que el hecho de no utilizar un sistema concreto de monitorización de la velocidad de marcha durante el entrenamiento domiciliario mediante caminata, pudo hacer que no se alcanzara la intensidad prescrita, al igual que en el estudio de Dias y col., antes mencionado (298). 
En cuanto a la calidad de vida, en nuestro estudio también se redujo la puntuación total del SGRQ por encima de la MCID establecida en 4 puntos (288). Este hallazgo es coherente con los reportados por otros investigadores que analizaron calidad de vida mediante el SGRQ tras la aplicación de RR domiciliaria (103-105). En todos ellos se observa una reducción en la puntuación total del cuestionario de más de 5 puntos. A diferencia de lo observado en el EST, parece que la intensidad de los programas no tuvo influencia en la calidad de vida, ya que mejoras relevantes fueron observadas en programas de baja intensidad, como el de Pomidori y col., antes indicado (105), o el de Sousa y col. (104), en el que los individuos entrenaban dos días por semana, 30 minutos al día, durante 12 semanas, a una intensidad correspondiente a un nivel de disnea moderada.

Por último, indicar que la ausencia de cambios en la percepción de disnea basal en nuestro estudio, no es coincidente con lo reportado en otras investigaciones que analizaban programas domiciliarios de RR $(101,104,105)$. Las discrepancias pueden achacarse a las características de los sujetos o a la duración de los programas. En el caso del estudio de Resqueti y col. (101), los sujetos analizados eran EPOC severos y muy severos que recibieron un PRR que incluía entrenamiento inspiratorio, hecho que también pudo influir. Por su parte, los estudios de Sousa (104) y Pomidori (105), utilizaron programas de más larga duración que el nuestro, aunque de menor intensidad, pero reportaron diferencias significativas en la disnea basal tras las intervenciones. En cualquier caso, la escala de disnea empleada pudo no representar adecuadamente los cambios, ya que según un reciente Consenso de Expertos sobre evaluación de disnea/síntomas en la EPOC (300), la Escala MRC es demasiado simple y dependiente del nivel de actividad, lo que hace que pueda no representar cambios tras el tratamiento.

\section{Efectos a medio y largo plazo}

A los tres meses de finalizar el programa, los sujetos del grupo experimental seguían mejorando ligeramente los resultados del EST. Esto puede deberse a que los pacientes continuaran con el entrenamiento establecido. En los nueve meses siguientes comenzó un declive lento en la distancia y el tiempo alcanzados en el EST, y al final del seguimiento, los resultados eran prácticamente iguales a los obtenidos tras la intervención.

No hemos encontrado estudios que analicen la duración de los efectos tras programas de RR domiciliaria similares al nuestro, evaluados mediante el EST. No obstante, podemos resaltar el estudio de Liu y col. (102), mencionado previamente, en el que se aplicaba un programa basado en marcha regulada mediante una aplicación musical. En éste se realizó un seguimiento de nueve meses tras la finalización del programa, efectuándose un control telefónico trimestral. No se observó una pérdida significativa en la distancia del IST, lo cual no puede achacarse a la mínima intervención efectuada, sino más bien al hecho de que los sujetos continuaban entrenando por su cuenta (la adhesión fue del 92\%). A pesar de que el protocolo de Liu fue distinto al nuestro, creemos que en ambos casos, el aporte de unas pautas claras de ejercicio a los pacientes y el empleo de un sistema de feed-back (aplicación musical o podómetro), fueron determinantes para que los sujetos mantuvieran el entrenamiento autónomo.

Nuestros resultados nos llevan a considerar que la prescripción de ejercicio mediante caminata pautada pudo ser útil para el mantenimiento de los efectos, como también demostraron otras investigaciones. En el estudio de du Moulin y col. (133), aplicando un mantenimiento de seis meses en el que los sujetos debían caminar a diario tres series de 15 minutos, a una velocidad equivalente al $125 \%$ de la alcanzada en un $6 \mathrm{MWT}$, utilizando un podómetro como sistema de 
feed-back y con control telefónico mensual, observaron que la distancia en el 6MWT mejoró durante este período y se mantuvo en los seis meses siguientes.

Por tanto, parece que el hecho de establecer objetivos concretos de cómo y cuánto deben salir a caminar con finalidad de entrenamiento es importante para aumentar la adhesión de los pacientes. Investigaciones que incorporaban recomendaciones genéricas de caminar a diario no reportaron tan buenos resultados en cuanto al mantenimiento de la capacidad de ejercicio $(130,134,135)$. En el estudio de Steele y col. (135) aplicaron un mantenimiento de 12 semanas tras un programa ambulatorio de RR de ocho semanas a pacientes EPOC severos, consistente en la recomendación de caminar lo máximo posible, sin pauta concreta de intensidad y tiempo. Se llevó a cabo un control telefónico semanal y se les aportó un podómetro. En el período de seguimiento se observó un declive en la distancia del 6MWT y a los 12 meses, la pérdida de los beneficios era casi completa. Igualmente, Linneberg y col. (130), incluyendo sesiones presenciales periódicas y registro en un diario de actividad durante un seguimiento de nueve meses tras su programa ambulatorio, reportaron que los resultados sobre la distancia en el EST declinaban a partir de los seis meses. Por su parte, Heppner y col. (134) tampoco consiguieron el mantenimiento de los resultados en la distancia del 6MWT tras 12 meses de un programa ambulatorio de nueve semanas. Este programa consistió en la simple prescripción de caminar a diario, control telefónico semanal y en consulta una vez al mes, durante seis meses. Observaron además, que en los individuos que caminaban más a diario tampoco se incrementaba la duración de los efectos sobre la capacidad de ejercicio, por lo que el hecho de mantenerse más activos no es suficiente para la permanencia del efecto entrenamiento, sino que es necesario combinarlo con la realización de ejercicio a la intensidad adecuada. Esta doble actuación fue la que pretendimos llevar a cabo en nuestro estudio.

Otro hallazgo interesante reportado en el Estudio 2 fueron las diferencias obtenidas al segmentar el grupo experimental según la clasificación GOLD (43). Los sujetos con GOLD I-II presentaron más incremento en el EST a los tres meses en relación al registro basal, pero el declive hasta los 12 meses fue mayor. Esto hace pensar que los individuos más leves mantuvieron la adhesión al programa a medio plazo, pero probablemente, el hecho de presentar un mejor estado funcional y no percibir los beneficios de la actividad física regular, hiciera que la adherencia se perdiera más en los meses siguientes. Por el contrario, en los sujetos más severos, al percibir los beneficios reportados por el entrenamiento, mantuvieron a largo plazo la continuidad en las pautas prescritas. En cualquier caso, sería necesario analizar una muestra más amplia para establecer conclusiones.

En cuanto a la calidad de vida, observamos que continuó mejorando en el grupo experimental en los tres primeros meses del seguimiento y se mantuvo hasta el final. Pensamos que esta mejoría puede deberse a que realmente la percepción de cambios no es tan inmediata y se materializa más claramente un poco después de finalizar una intervención. En el estudio de Spencer y col. (129) , en el que se evaluó la calidad de vida mediante el SGRQ, también observaron un mantenimiento de los efectos tras la RR durante su seguimiento de 12 meses, al aplicar pautas concretas de caminar y la cumplimentación de un diario de actividad.

Por otro lado, destacar que en el estudio de Pomidori y col. (105), al que nos hemos referido con anterioridad, tras seis meses de la finalización de los programas sin estrategias de mantenimiento específicas, los resultados sobre la capacidad de ejercicio, la calidad de vida y la disnea, perduraron sólo en el grupo que utilizó un metrómetro como sistema de feed-back 
para el control del ritmo de caminata durante el entrenamiento. Esto refuerza la efectividad del uso de sistemas que permitan a los pacientes llevar a cabo un control preciso de la intensidad del entrenamiento.

Por último, indicar que en el grupo experimental se observó una ligera tendencia decreciente en el número de exacerbaciones en el período de seguimiento en relación a los 12 meses previos, aunque estas diferencias no fueron significativas. Van Wettering y col. (99) tampoco hallaron cambios en las exacerbaciones durante un periodo de 20 meses tras un PRR domiciliario de cuatro meses, que incluía una sesión de mantenimiento mensual. Los autores afirman que puede deberse a que las sesiones educativas aplicadas al grupo control mejoraron las estrategias de manejo de la enfermedad de los pacientes, lo que les permitió detectar precozmente y afrontar de forma más eficaz las reagudizaciones (99). En nuestro estudio, este efecto también pudo ser responsable de la ausencia de diferencias entre los grupos. Por otro lado, el registro del número de episodios de reagudización mediante la información aportada por los pacientes pudo no ser suficientemente discriminativo, así como el hecho de que los sujetos eran estables (la mayoría presentaron menos de 2 exacerbaciones al año) e incluso, el tamaño de la muestra, pudieron ser determinantes.

A la vista de estos resultados, podemos establecer que el programa de RR domiciliaria aplicado fue eficaz para mejorar la tolerancia al ejercicio y la calidad de vida en los sujetos de la muestra. Parece que el entrenamiento mediante caminata a alta intensidad, el aporte de pautas precisas y el empleo de podómetros como sistema de feed-back pudieron tener un efecto en que los pacientes continuaran entrenando, puesto que la intervención durante el período de seguimiento fue mínima. Del mismo modo, la actuación asociada al programa, dirigida a modificar los hábitos de actividad física de los pacientes, pudo influir positivamente. El tamaño de la muestra del estudio superó el tamaño muestral óptimo para la variable principal, la tolerancia al ejercicio, lo que aumenta la fortaleza de nuestros resultados. Así mismo, en el análisis de sensibilidad llevado a cabo, no hubo diferencias en relación al análisis por protocolo, lo que indica que las pérdidas no tuvieron influencia en los hallazgos.

\section{La modificación del nivel de actividad física en la EPOC y su influencia sobre el estado funcional de los pacientes}

En el Estudio 1 de esta tesis no se observaron cambios en el número de horas de ejercicio semanal que indicaban realizar los pacientes, tras la aplicación de los tres programas de RR ambulatoria.

Por el contrario, en el Estudio 2, se ha demostrado un incremento en el número de pasos/día y en la puntuación del Cuestionario de Actividad Física Baecke Modificado tras la aplicación del protocolo experimental, manteniéndose estos resultados a los 3 y 12 meses. Así mismo, se halló una asociación significativa del incremento en estas dos variables con la mejoría en la distancia y el tiempo alcanzados en el eST, así como con la reducción en la puntuación total del cuestionario de calidad de vida SGRQ.

\section{Eficacia de las intervenciones en la modificación} del nivel de actividad física en la EPOC

La ausencia de cambios en el número de horas que los pacientes indicaban que realizaban de ejercicio físico moderado por semana en el Estudio 1, es coherente con otros estudios que tampoco hallaron cambios en el nivel de AF tras programas ambulatorios de RR 
$(150,155,208,217,301)$. En nuestro caso, el añadir entrenamiento de fuerza o específico de los músculos respiratorios no aportó nada adicional al entrenamiento aeróbico aislado. No obstante, la herramienta para medir el nivel de AF no fue la más apropiada, al tratarse de un sistema subjetivo $(152,153,302,303)$.

En el Estudio 2, el número de pasos/día aumentó alrededor de un 45\% tras la intervención, continuó incrementándose modestamente a los tres meses y presentó una ligera tendencia descendente en los nueve meses siguientes, (esta variable permanecía aún un $25 \%$ más alta al final del estudio que en el registro basal). De este modo, en el grupo experimental se alcanzó la meta de los 10.000 pasos/día tras la intervención y a los tres meses, y de casi 9.000 pasos/día a los 12 meses. Del mismo modo, la puntuación total del Cuestionario Baecke Modificado se incrementó moderadamente tras la intervención en el grupo experimental y siguió aumentando en los tres meses siguientes, de forma más relevante, lo que refleja el cambio comportamental acontecido. En los nueve meses posteriores, se observa un cierto declive, similar a lo ocurrido con el número de pasos/día, pero aún la variable se mantenía en valores superiores a los alcanzados post-intervención. En el momento de máximo incremento de la puntuación del cuestionario (3 meses post-intervención), se superó la barrera de los 9 puntos, que determina la diferencia entre perfil sedentario y con actividad moderada (285).

El incremento en los pasos/día en nuestro estudio fue mayor al reportado por Bravata y col. (225) en su metanálisis sobre estrategias de incremento de AF en la población general (hallaron un incremento medio de 2.490 pasos/día) y se mantuvo prácticamente en este nivel tras el seguimiento. Así mismo, los resultados fueron similares a los reportados por Chan y col. (230), al aplicar un programa de incremento de AF en sujetos sanos. Estos autores también basaron su intervención en el establecimiento de logros individualizados y la utilización de un podómetro como feed-back. Hallaron un incremento del $48 \%$ en el número de pasos/ día y del $21 \%$ tras un periodo de 12 meses de seguimiento. Por tanto, podemos afirmar que los pacientes EPOC de nuestro estudio fueron capaces de alcanzar resultados similares a la población sana.

En pacientes EPOC también se han realizado algunas investigaciones con resultados similares $(241,248)$. Cruz y col. (248) aplicaron un plan de incremento de actividad asociado a un programa ambulatorio de RR de 12 semanas, que consistía en el establecimiento de logros individuales y secuenciales dirigidos a alcanzar un objetivo final de caminar 10.000 pasos/día, aumentando su pauta de caminata semanal en unos 800 pasos/día. Utilizaron como sistema de retroalimentación un podómetro y evaluaban lo alcanzado semanalmente en una sesión presencial. En este estudio, además se añade un período de mantenimiento de tres meses tras la finalización del PRR, en el que se llevaba a cabo un control telefónico semanal durante el primer mes y quincenal durante los dos siguientes. Los sujetos analizados tenían un número de pasos/día basales similar a nuestra muestra y reportaron una mejoría parecida (incremento de 2.900 pasos/día al finalizar el programa y de 2.600 tras el período de mantenimiento). Por su parte, De Blok y col. (241) aplicaron un programa equivalente a sujetos que estaban recibiendo un PRR ambulatorio de ocho semanas, con establecimiento individualizado de logros, retroalimentación mediante un podómetro y sesiones motivacionales a lo largo del programa. En este estudio, el incremento en el número de pasos fue del 69\%, aunque no alcanzó los 2.000 pasos/día puesto que se trataba de sujetos severos con menor nivel de actividad previa que los del estudio de Cruz o el nuestro. 
Otras investigaciones han reportado resultados menos importantes al aplicar programas de incremento de actividad basados en el uso de podómetros, independientes de la realización de entrenamiento físico (148,236-239). La adición del programa de modificación comportamental a un programa de RR ambulatorio como los de Cruz y de Blok antes indicados $(241,248)$, o autoadministrados como el nuestro, puede ser un factor a tener en cuenta para optimizar los resultados. Esto podría deberse a que los pacientes reducen el miedo a la práctica de ejercicio al realizar un PRR (211) o a que el aumento en la capacidad de ejercicio y la reducción de los síntomas asociados al esfuerzo, como consecuencia del entrenamiento, favorece que los pacientes hagan más actividad por su cuenta, ya que el miedo a la práctica deportiva y la aparición de disnea con el ejercicio son unas de las principales barreras de limitación de actividad autorreportadas por los pacientes (97). Esta reflexión es coincidente con las conclusiones de la reciente revisión de Mantoani y col. (212), que indican que para aumentar la eficacia de las intervenciones de modificación del nivel de AF en pacientes EPOC, es recomendable asociar entrenamiento físico de alta intensidad y utilizar un sistema de monitorización objetivo.

En la publicación de Altenburg y col. (236), los sujetos del grupo experimental aumentaron el número de pasos/día en alrededor de 500 y en el estudio de Hospes y col. (148) en unos 800. En ambos estudios, la muestra analizada fue muy similar a la nuestra, especialmente en cuanto al número de pasos basales, por lo que las diferencias deberían achacarse más bien a las características de los programas, que a pesar de que tenían similitudes con el nuestro (utilizaban podómetros como sistema de feed-back y una estrategia de cambio comportamental basada en el establecimiento de logros), las sesiones de control eran menos frecuentes (cada dos o tres semanas), no establecían un objetivo final claro y los objetivos parciales eran totalmente individualizados, sin un estándar general orientativo. Esta mayor flexibilidad, junto con el no añadir entrenamiento físico al programa de incremento de actividad, pudieron influir en obtener resultados más modestos. Además, en el estudio de Altenburg y col. (236) se produjo una pérdida de los resultados durante el período de seguimiento, por lo que su estrategia no provocó cambios en los hábitos de los pacientes a largo plazo, a diferencia de lo observado en nuestro estudio.

Por su parte, Moy y col. desarrollaron un programa de 16 semanas basado en un registro podométrico y el uso de una herramienta informática (página web) que diseñaron específicamente y aplicaron en diversas poblaciones de EPOC estadounidenses (237-239). En estos programas, los pacientes descargaban semanalmente el registro de pasos en su ordenador y la aplicación informática les devolvía información sobre el nuevo objetivo para la semana siguiente, junto con mensajes motivacionales. En estos estudios, carentes de grupo control, los pacientes aumentaron su nivel de caminata habitual, ligeramente por debajo de 1.000 pasos/ día, excepto en el publicado en 2012 (238), en que se alcanzó un incremento en 1.200 pasos/ día, al añadir control telefónico semanal. Estos resultados son inferiores a los nuestros, pero hay que indicar que la población de estudio era más inactiva, ya que en ningún caso superó los 3.500 pasos/día de media en el registro basal. Otra posible explicación de las diferencias podría ser el menor control de los sujetos, puesto que al añadir las llamadas telefónicas se potencian los efectos (239).

El hecho de dar indicaciones de ejercicio precisas a los pacientes de nuestro estudio, también pudo ser un factor relevante en los buenos resultados obtenidos, puesto que el aporte de un podómetro y la recomendación genérica de caminar más puede no ser suficiente 
$(135,148,241)$. Steele y col. (135), al proporcionar un podómetro y recomendaciones generales durante 12 semanas a sujetos que habían realizado un PRR ambulatorio, no hallaron cambios en el nivel de AF evaluado mediante acelerometría. Así mismo, en el estudio de De Blok y col. (241) y en el de Hospes y col. (148), aportaron un podómetro al grupo control, observando que esta actuación no supuso cambios significativos en el número de pasos/día. En contraposición, Mendoza y col. (242) sí encontraron un aumento significativo y relevante (3.000 pasos/día) al aplicar un programa de incremento de actividad de tres meses, en el que la única diferencia entre los grupos era que los sujetos del grupo experimental contaban con un podómetro y debían anotar sus pasos diarios. No obstante, la muestra analizada en este estudio presentaba poca afectación ventilatoria ( $\mathrm{FEV}_{1}$ del $66 \%$ del valor teórico) y no se evaluó la permanencia del efecto a más largo plazo.

Por último, podemos pensar que los sujetos de nuestro estudio no sólo aumentaron su nivel de actividad habitual, sino que mantuvieron el hábito de caminar deprisa durante cierto tiempo al día, tal y como debían realizar durante el programa de RR autoadministrado, lo que pudo influir en el mantenimiento de la capacidad de ejercicio que se ha descrito en el apartado anterior. Puesto que se ha reportado que un adulto caminando a velocidad moderada alcanza 1.000 pasos en 10 minutos (304), si consideramos que los sujetos del grupo experimental aumentaron en alrededor de 3.000 pasos/día, es esperable que parte de éstos correspondieran a una caminata a cierta velocidad durante 30 minutos. A diferencia de nuestros hallazgos, en el estudio COPE-Active (218), el incremento en 1.190 pasos/día que mantenían los pacientes tras un periodo de seguimiento de 12 meses, no consiguió evitar el declive en el EST. Esto refuerza la afirmación de que caminar más no es suficiente, sino que hay que garantizar que además, realicen algún tipo de actividad moderada (154), lo que puede justificar los resultados de nuestra doble intervención.

\section{Factores que pueden determinar el efecto de las intervenciones} de incremento de actividad en los pacientes EPOC

Las condiciones climatológicas pueden ser un factor limitante para la práctica de ejercicio por los pacientes EPOC $(97,305)$ y la población general $(306)$, pero en el Estudio 2 no hallamos diferencias significativas entre los sujetos que habían recibido el programa en primavera-verano y los que lo habían hecho en otoño-invierno. Esto puede deberse a que los períodos más fríos no son lo suficientemente duros en Madrid para que los pacientes permanezcan sin salir de casa o bien, el tamaño muestral no permitió establecer discrepancias. Otros estudios, con muestras más grandes y realizados en zonas geográficas con peores condiciones climatológicas, sí hallaron un menor número de pasos en los meses de otoño e invierno $(168,171)$. En el estudio de Moy y col. (168) observaron una tendencia de mayor actividad en primavera que en verano, en verano que en otoño y en otoño que en invierno, pero las diferencias no fueron significativas.

El tamaño muestral también puede justificar el no haber encontrado resultados distintos al segmentar la muestra por edad o severidad de la enfermedad, a pesar de que se ha demostrado que el nivel de AF se reduce con la edad $(157,172-176)$ y en los estadios GOLD III y IV $(151,157,168,183-185)$. Así mismo, el bajo número de sujetos con OCD en nuestra muestra no permitió obtener diferencias significativas en los resultados de actividad entre éstos y los que no necesitaban oxígeno, lo que es coherente con el estudio de Nyssen y col. (174), aunque también se ha reportado que la OCD puede reducir el número de pasos en los pacientes EPOC (171), así como las horas fuera de casa (176). 


\section{Asociación entre la modificación del nivel de actividad física y la capacidad funcional de los pacientes EPOC}

En el Estudio 2 hallamos una correlación significativa entre el incremento en el número de pasos/día y en la puntuación del Cuestionario Baecke, con el aumento en la distancia y el tiempo alcanzados en el EST y la reducción en la puntuación del SGRQ, tanto a los 3 como a los 12 meses de seguimiento. Esto indica que el cambio en el nivel de AF que experimentaron los sujetos tuvo influencia en la mejora en la capacidad de ejercicio y la calidad de vida a medio y largo plazo. Sin embargo, no se demostró asociación con la disnea ni las exacerbaciones.

Existe una fuerte evidencia acerca de la correlación entre el nivel de AF en los pacientes EPOC y su capacidad de ejercicio $(152,159,160,162,163,169-172,179,181,182,184,188-190)$, pero la asociación entre la variación en estas variables ha sido analizada en un número menor de investigaciones, obteniendo resultados similares a los nuestros en todos los casos $(172,307)$. En el estudio de Zwerink y col, realizado sobre la cohorte COPE-Active (172), hallaron una correlación significativa entre la modificación en el número de pasos/día y en los resultados del EST, aunque menos potente que la nuestra $(r<0,5)$. Las diferencias podrían deberse a que el incremento en el número de pasos y en la capacidad de ejercicio también fue más modesto que en nuestro estudio, tal y como se ha expuesto antes.

En cuanto a calidad de vida, como sea expuesto anteriormente, se ha encontrado una correlación significativa entre ésta y el número de pasos/día $(164,169,170)$ o el tiempo dedicado a caminar deprisa durante el día (206). No obstante, tampoco hay muchos estudios que analicen la asociación entre el cambio de estas variables. Moy y col., en su publicación de 2015 (236), hallaron una correlación significativa entre el incremento en los pasos experimentado por los sujetos que siguieron el programa Training Health Steps (THS) y la reducción en la puntuación del SGRQ, aunque no ofrecen resultados a largo plazo. Por su parte, Esteban y col. (207) también encontraron una asociación significativa e inversa entre el cambio en el tiempo dedicado a caminar a la semana y la puntuación del SGRQ durante un período de seguimiento de cinco años. Así mismo, Waschki y col. (173) han demostrado una correlación potente e inversa entre el incremento en el PAL a lo largo de tres años y la puntuación total del SGRQ.

Aunque la evidencia mayoritaria parece ser consistente con nuestros hallazgos, en el estudio del Altenburg y col. (236) no encontraron asociación entre el aumento en el número de pasos que alcanzaron los sujetos que realizaron su programa de incremento de actividad y los cambios en el 6MWT o el SGRQ. El hecho de que los cambios acontecidos tras el programa fueron leves aunque significativos puede ser determinante. Esto nos lleva a pensar que para influir en la capacidad funcional de los sujetos, hay que aplicar intervenciones que optimicen los resultados sobre el nivel de actividad fisica.

En relación a la disnea basal, hay cierta controversia a la hora de establecer su asociación con el nivel de AF en la EPOC, encontrándose estudios con correlación significativa $(162,163,168,171,179,184,189)$ y otros sin correlación $(161,170,174,175)$. Por otro lado, no hemos hallado publicaciones que analicen la variación entre ambas variables, por lo que no podemos establecer comparaciones con nuestros resultados. No obstante, la ausencia de asociación entre la variación en AF y en disnea en nuestra muestra puede achacarse a la herramienta utilizada para evaluar la disnea basal.

Por último, la ausencia de asociación entre la variación en el nivel de AF y las exacerbaciones durante el período de seguimiento en nuestro estudio, no es coherente con las numerosas 
investigaciones que hallaron una correlación entre el nivel de actividad de los pacientes EPOC y los ingresos hospitalarios por reagudización en los meses consecutivos $(170,178,182,202-204)$. Tampoco con los estudios que evaluaron, como nosotros, la influencia del cambio en el nivel de ejercicio en la aparición de episodios de este tipo $(186,197)$. Esteban y col. (197) encontraron una correlación significativa entre el incremento en el tiempo que dedicaban a caminar durante dos años consecutivos y la aparición de exacerbaciones en los tres años siguientes. Durheim y col. (186) reportaron unos resultados similares en los sujetos de una cohorte de más de 300 pacientes con EPOC que aumentaron el número de pasos a lo largo de cuatro años. Las diferencias con nuestros resultados podrían radicar en el hecho del menor tamaño muestral de nuestro estudio en relación a estos dos y el período de seguimiento, también menos prolongado. Creemos que para alcanzar resultados concluyentes acerca de una variable tan relacionada con la evolución de la enfermedad, es necesario llevar a cabo seguimientos más largos a un año y analizar muestras más numerosas.

En resumen, podemos afirmar que el programa aplicado en el Estudio 2 fue eficaz para aumentar el nivel de AF de los sujetos y mantenerlo hasta 12 meses. El establecer objetivos secuenciales y claros, el control frecuente del nivel de cumplimiento y el sistema de monitorización sencillo mediante un podómetro, modificaron los hábitos de los pacientes, y el asociar un programa de entrenamiento autoadministrado de alta intensidad pudo potenciar los efectos de adhesión. Así mismo, el incremento en el nivel de AF tuvo influencia en los cambios en la capacidad de ejercicio y la calidad de vida. Los buenos resultados obtenidos con la intervención parecen magnificar esta asociación en relación a otras investigaciones. Sin embargo, el bajo tamaño muestral, la herramienta de evaluación utilizada y el período de seguimiento no demasiado prolongado, pudieron contribuir a que no se hallaran asociaciones con la disnea basal y las exacerbaciones. Estos resultados no se vieron afectados por las pérdidas (el análisis de sensibilidad no ofreció diferencias con el análisis por protocolo).

\section{Limitaciones de los estudios}

Una de las principales limitaciones de los estudios incluidos en esta tesis es el tamaño muestral, especialmente en el Estudio 1 y en el análisis de la variable nivel de AF en el Estudio 2. En ambos casos, el número de sujetos analizados se encuentra por debajo del tamaño muestral óptimo, por lo que los resultados no pueden extrapolarse. En el caso del estudio observacional, Estudio 1, se analizaron a todos los sujetos de los que disponíamos de datos completos y que cumplían los criterios de inclusión. Es importante recordar que se trató de un análisis retrospectivo de pacientes incluidos en los programas de Rehabilitación Respiratoria de nuestro Centro entre 2010 y 2012. En el ECA, la aparición de problemas en el proceso de reclutamiento nos obligó a reducir el número de sujetos incluidos para no demorar en exceso la finalización del trabajo de campo. No obstante, se superó el tamaño muestral adecuado para la variable de capacidad de ejercicio, por lo que la potencia de los resultados en esta variable está garantizada. En este sentido y comparando con investigaciones similares, muchos estudios cuentan con muestras equivalentes.

Otra limitación de ambos estudios es la herramienta utilizada para evaluar la disnea basal, la Escala mMRC, ya que, como se ha indicado, parece que no es suficientemente sensible para detectar cambios, especialmente con muestras pequeñas.

Así mismo, el sistema de registro del nivel de AF en el Estudio 1 no era el más adecuado, pero era la única información de la que disponíamos. 
Por otro lado, en el Estudio 2, a pesar de que el período de seguimiento de 12 meses es un valor añadido frente a otros estudios, hubiera sido interesante haberlo prolongado para analizar la duración del cambio comportamental a más largo plazo y observar si se modifican los resultados sobre exacerbaciones. De todos modos, éste es un período habitual de seguimiento a largo plazo, que en la mayoría de los estudios ha permitido demostrar cambios en el comportamiento de los pacientes con EPOC. 


\section{CONCLUSIONES}

1. Los PRR ambulatorios de ocho semanas, aplicados en el tratamiento habitual de pacientes EPOC en nuestro Centro, mejoran la capacidad de ejercicio, la disnea basal y la PIM-PEM (cuando se incluye IMT + EMT). Los resultados se potencian al incorporar entrenamiento de fuerza y específico de músculos respiratorios al entrenamiento aeróbico, aunque no se hallaron diferencias significativas entre los programas. No se observó efecto en la reducción de las exacerbaciones posteriores ni en el incremento del número de horas semanales de actividad física.

2. Estos efectos se pierden prácticamente en su totalidad tras un período de seguimiento de 12 meses sin estrategias de mantenimiento, independientemente de factores como el sexo, la edad, el hábito tabáquico, el uso de OCD, la severidad de la enfermedad o el IMC. Se detectó un declive más lento, no significativo, al combinar con entrenamiento de fuerza periférica y de los músculos respiratorios.

3. El incremento en la distancia del 6MWT tras la RR puede ser un factor pronóstico de declive en la capacidad de ejercicio de los pacientes.

4. Un programa de RR domiciliario de ocho semanas basado en caminatas a alta velocidad, asociado a un plan de incremento de actividad, utilizando un podómetro como sistema de retroalimentación, es eficaz en mejorar la capacidad de ejercicio, el nivel de AF y la calidad de vida en pacientes EPOC y mantener los resultados durante 12 meses.

5. Estos resultados pueden achacarse a cambios relacionados con el incremento de los niveles de actividad física y al mantenimiento a largo plazo del entrenamiento. Los factores que han podido propiciar el cambio son: la aplicación simultánea de la doble actuación, la optimización de los resultados del PRR al emplear un entrenamiento de alta intensidad y basado en caminar, el establecimiento de pautas concretas de progresión en ambas intervenciones y el empleo de un sistema de monitorización objetiva y fácil de utilizar por los pacientes.

6. El cambio en el nivel de actividad física a lo largo del tiempo presentó una correlación significativa con la mejora en la capacidad de ejercicio y la calidad de vida, aunque no se encontró asociación con la disnea o el número de exacerbaciones.

7. Sería necesario realizar estudios con un seguimiento más prolongado para determinar si el cambio comportamental se mantiene a más largo plazo y observar si hay variación en las exacerbaciones. En este sentido, sería interesante explorar qué factores pueden influir en el cambio de comportamiento. 


\section{PERSPECTIVAS DE FUTURO}

Los resultados positivos obtenidos en el Estudio 2 inducen a evaluar el programa sobre una muestra más amplia y utilizando sistemas complementarios de registro de las variables, así como el análisis de otros aspectos, como la respuesta inflamatoria sistémica. Sería interesante evaluar la tolerancia al esfuerzo mediante una prueba incremental en cicloergómetro para determinar el efecto del programa en parámetros como el $\mathrm{VO}_{2}$ max. El registro de otros indicadores de movimiento y actividad además de los pasos, y el empleo de los nuevos cuestionarios validados que analizan el grado de dificultad percibida por los pacientes al realizar actividad física, también aportaría valor añadido.

Del mismo modo, realizar un seguimiento más prolongado permitiría confirmar si el cambio comportamental es estable y su influencia en las exacerbaciones. Todos los estudios actuales insisten en la importancia del cambio de comportamiento para que los pacientes incorporen estilos de vida más saludables. De este modo, sería interesante ahondar en la identificación de los factores que propician el cambio de hábitos en los pacientes

Por otro lado, de las conclusiones de esta tesis se extraen algunos retos que pueden servir como líneas de investigaciones futuras. Entre ellos, es necesario ampliar la evidencia acerca de:

- Las diferencias en la duración de los efectos tras la aplicación de diversas modalidades de programas de RR ambulatoria.

- La influencia de los PRR autoadministrados en las exacerbaciones de los pacientes EPOC.

- Las estrategias más eficaces para mantener los efectos tras la RR y conseguir cambios comportamentales duraderos en los pacientes EPOC, dirigidos a aumentar su nivel de actividad física habitual y la adhesión por la práctica de ejercicio. 


\section{BIBLIOGRAFÍA}

1. Global Strategy for Diagnosis, Management, and Prevention of COPD - 2016 [Internet]. Global Initiative for Chronic Obstructive Lung Disease - GOLD. [citado 8 de octubre de 2016]. Disponible en: http://goldcopd. org/global-strategy-diagnosis-management-prevention-copd-2016/

2. Miravitlles M, Soler-Cataluña JJ, Calle M, Molina J, Almagro P, Quintano JA, et al. Guía Española de la EPOC (GesEPOC). Tratamiento farmacológico de la EPOC estable. Arch Bronconeumol [Internet]. 2012 [citado 1 de octubre de 2016]; 48(7):247-57. Disponible en: http://www.archbronconeumol.org/es/ guia-espanolaepgesepoctratamiento/articulo/\$0300289612001159/

3. Celli BR, MacNee W, ATS/ERS Task Force. Standards for the diagnosis and treatment of patients with COPD: a summary of the ATS/ERS position paper. Eur Respir J. 2004;23(6):932-46.

4. Forey BA, Thornton AJ, Lee PN. Systematic review with meta-analysis of the epidemiological evidence relating smoking to COPD, chronic bronchitis and emphysema. BMC Pulm Med [Internet]. 2011 [citado 12 de marzo de 2017]; 11:36. Disponible en: http://dx.doi.org/10.1186/1471-2466-11-36

5. Fletcher C, Peto R. The natural history of chronic airflow obstruction. Br Med J. 1977;1(6077):1645-8.

6. Kohansal R, Martinez-Camblor P, Agustí A, Buist AS, Mannino DM, Soriano JB. The natural history of chronic airflow obstruction revisited: an analysis of the Framingham offspring cohort. Am J Respir Crit Care Med. 2009;180(1):3-10.

7. Løkke A, Lange P, Scharling H, Fabricius P, Vestbo J. Developing COPD: a 25 year follow up study of the general population. Thorax. 2006;61(11):935-9.

8. Yin P, Jiang CQ, Cheng KK, Lam TH, Lam KH, Miller MR, et al. Passive smoking exposure and risk of COPD among adults in China: the Guangzhou Biobank Cohort Study. Lancet Lond Engl. 2007;370(9589):751-7.

9. McCloskey SC, Patel BD, Hinchliffe SJ, Reid ED, Wareham NJ, Lomas DA. Siblings of patients with severe chronic obstructive pulmonary disease have a significant risk of airflow obstruction. Am J Respir Crit Care Med. 2001;164(8 Pt 1):1419-24.

10. Sørheim I-C, Johannessen A, Gulsvik A, Bakke PS, Silverman EK, DeMeo DL. Gender differences in COPD: are women more susceptible to smoking effects than men? Thorax. 2010;65(6):480-5.

11. Lawlor DA, Ebrahim S, Davey Smith G. Association of birth weight with adult lung function: findings from the British Women's Heart and Health Study and a meta-analysis. Thorax. de 2005;60(10):851-8.

12. Svanes C, Sunyer J, Plana E, Dharmage S, Heinrich J, Jarvis D, et al. Early life origins of chronic obstructive pulmonary disease. Thorax. 2010;65(1):14-20.

13. de Marco R, Accordini S, Marcon A, Cerveri I, Antó JM, Gislason T, et al. Risk factors for chronic obstructive pulmonary disease in a European cohort of young adults. Am J Respir Crit Care Med. 2011;183(7):891-7.

14. Hu G, Zhou Y, Tian J, Yao W, Li J, Li B, et al. Risk of COPD from exposure to biomass smoke: a metaanalysis. Chest. 2010;138(1):20-31.

15. Eisner MD, Anthonisen N, Coultas D, Kuenzli N, Perez-Padilla R, Postma D, et al. An official American Thoracic Society public policy statement: Novel risk factors and the global burden of chronic obstructive pulmonary disease. Am J Respir Crit Care Med. 2010;182(5):693-718.

16. Rodríguez E, Ferrer J, Martí S, Zock J-P, Plana E, Morell F. Impact of occupational exposure on severity of COPD. Chest. 2008;134(6):1237-43.

17. Prescott $E$, Lange $P$, Vestbo J. Socioeconomic status, lung function and admission to hospital for COPD: results from the Copenhagen City Heart Study. Eur Respir J. 1999;13(5):1109-14.

18. Silva GE, Sherrill DL, Guerra S, Barbee RA. Asthma as a risk factor for COPD in a longitudinal study. Chest. 2004;126(1):59-65.

19. Vos T, Flaxman AD, Naghavi M, Lozano R, Michaud C, Ezzati M, et al. Years lived with disability (YLDs) for 1160 sequelae of 289 diseases and injuries 1990-2010: a systematic analysis for the Global Burden of Disease Study 2010. Lancet Lond Engl. 2012;380(9859):2163-96. 
20. Buist AS, McBurnie MA, Vollmer WM, Gillespie S, Burney P, Mannino DM, et al. International variation in the prevalence of COPD (the BOLD Study): a population-based prevalence study. Lancet Lond Engl. 2007;370(9589):741-50.

21. Halbert RJ, Natoli JL, Gano A, Badamgarav E, Buist AS, Mannino DM. Global burden of COPD: systematic review and meta-analysis. Eur Respir J. 2006;28(3):523-32.

22. Atsou K, Chouaid C, Hejblum G. Variability of the chronic obstructive pulmonary disease key epidemiological data in Europe: systematic review. BMC Med [Internet]. [citado 6 de noviembre de 2016]; 9(1):7. Disponible en: http://bmcmedicine.biomedcentral.com/articles/10.1186/1741-7015-9-7

23. Miravitlles M, Soriano JB, García-Río F, Muñoz L, Duran-Tauleria E, Sanchez G, et al. Prevalence of COPD in Spain: impact of undiagnosed COPD on quality of life and daily life activities. Thorax. 2009;64(10):863-8.

24. Ancochea J, Miravitlles M, García-Río F, Muñoz L, Sánchez G, Sobradillo V, et al. Underdiagnosis of chronic obstructive pulmonary disease in women: quantification of the problem, determinants and proposed actions. Arch Bronconeumol. 2013;49(6):223-9.

25. WHO | Burden of COPD [Internet]. WHO. [citado 8 de octubre de 2016]. Disponible en: http://www. who.int/respiratory/copd/burden/en/

26. Lozano R, Naghavi M, Foreman K, Lim S, Shibuya K, Aboyans V, et al. Global and regional mortality from 235 causes of death for 20 age groups in 1990 and 2010: a systematic analysis for the Global Burden of Disease Study 2010. Lancet Lond Engl. 2012;380(9859):2095-128.

27. Patrones de mortalidad en España, 2008 - PatronesMortalidadEspana2013.pdf [Internet]. [citado 6 de noviembre de 2016]. Disponible en: http://www.msssi.gob.es/estadEstudios/estadisticas/estadisticas/ estMinisterio/mortalidad/docs/PatronesMortalidadEspana2013.pdf

28. Indicadores clave del Sistema Nacional de Salud [Internet]. [citado 8 de octubre de 2016]. Disponible en: http://inclasns.msssi.es/main.html

29. ERS - Respiratory health and disease in Europe [Internet]. [citado 6 de noviembre de 2016]. Disponible en: http://www.erswhitebook.org/

30. CUB EPOC - EstrategiaEPOCSNS.pdf [Internet]. [citado 1 de octubre de 2016]. Disponible en: http://www. msc.es/organizacion/sns/planCalidadSNS/docs/EstrategiaEPOCSNS.pdf

31. Agusti A, Vestbo J. Chronic Obstructive Pulmonary Disease Phenotypes: Current Rather than Future Perspectives. Am J Respir Crit Care Med [Internet]. 2012 [citado 2 de enero de 2017];185(5):590-590. Disponible en: http://www.atsjournals.org/doi/full/10.1164/ajrccm.185.5.590

32. Casanova C, de Torres JP, Aguirre-Jaíme A, Pinto-Plata V, Marin JM, Cordoba E, et al. The progression of chronic obstructive pulmonary disease is heterogeneous: the experience of the BODE cohort. Am J Respir Crit Care Med. 2011;184(9):1015-21.

33. de-Torres JP, Marín JM, Pinto-Plata V, Divo M, Sanchez-Salcedo P, Zagaceta J, et al. Is COPD a Progressive Disease? A Long Term Bode Cohort Observation. PLoS ONE [Internet]. 2016 [citado 12 de marzo de 2017];11(4). Disponible en: http://www.ncbi.nlm.nih.gov/pmc/articles/PMC4839642/

34. Miravitlles M, Soler-Cataluña JJ, Calle M, Molina J, Almagro P, Quintano JA, et al. Spanish guideline for COPD (GesEPOC). Update 2014. Arch Bronconeumol. 2014;50 Suppl 1:1-16.

35. Miravitlles M. Cough and sputum production as risk factors for poor outcomes in patients with COPD. Respir Med. 2011;105(8):1118-28.

36. Andreassen $\mathrm{H}$, Vestbo J. Chronic obstructive pulmonary disease as a systemic disease: an epidemiological perspective. Eur Respir J Suppl. 2003;46:2s-4s.

37. Agustí AGN. Systemic effects of chronic obstructive pulmonary disease. Proc Am Thorac Soc. 2005;2(4):367-370; discussion 371-372.

38. Barnes PJ, Celli BR. Systemic manifestations and comorbidities of COPD. Eur Respir J. 2009;33(5):1165-85. 
39. Divo M, Cote C, de Torres JP, Casanova C, Marin JM, Pinto-Plata V, et al. Comorbidities and risk of mortality in patients with chronic obstructive pulmonary disease. Am J Respir Crit Care Med. 2012;186(2):155-61.

40. Chatila WM, Thomashow BM, Minai OA, Criner GJ, Make BJ. Comorbidities in chronic obstructive pulmonary disease. Proc Am Thorac Soc. 2008;5(4):549-55.

41. Vanfleteren LEGW, Spruit MA, Groenen M, Gaffron S, van Empel VPM, Bruijnzeel PLB, et al. Clusters of comorbidities based on validated objective measurements and systemic inflammation in patients with chronic obstructive pulmonary disease. Am J Respir Crit Care Med. 2013;187(7):728-35.

42. García-Olmos L, Alberquilla Á, Ayala V, García-Sagredo P, Morales L, Carmona M, et al. Comorbidity in patients with chronic obstructive pulmonary disease in family practice: a cross sectional study. BMC Fam Pract [Internet]. 2013 [citado 2 de enero de 2017]; 14:11. Disponible en: http://dx.doi.org/10.1186/1471-2296-14-11

43. Pauwels RA, Buist AS, Calverley PM, Jenkins CR, Hurd SS, GOLD Scientific Committee. Global strategy for the diagnosis, management, and prevention of chronic obstructive pulmonary disease. NHLBI/WHO Global Initiative for Chronic Obstructive Lung Disease (GOLD) Workshop summary. Am J Respir Crit Care Med. 2001;163(5):1256-76.

44. Celli BR, Cote CG, Marin JM, Casanova C, Montes de Oca M, Mendez RA, et al. The body-mass index, airflow obstruction, dyspnea, and exercise capacity index in chronic obstructive pulmonary disease. N Engl J Med. 2004;350(10):1005-12.

45. Soler-Cataluña JJ, Martínez-García MÁ, Sánchez LS, Tordera MP, Sánchez PR. Severe exacerbations and BODE index: Two independent risk factors for death in male COPD patients. Respir Med [Internet]. 2009 [citado 27 de julio de 2016]; 103(5):692-9. Disponible en: /article/S0954-6111(08)00449-6/abstract

46. Vestbo J, Hurd SS, Rodriguez-Roisin R. The 2011 revision of the global strategy for the diagnosis, management and prevention of COPD (GOLD)--why and what? Clin Respir J. 2012;6(4):208-14.

47. Nici L, Donner C, Wouters E, Zuwallack R, Ambrosino N, Bourbeau J, et al. American Thoracic Society/ European Respiratory Society statement on pulmonary rehabilitation. Am J Respir Crit Care Med. 2006;173(12):1390-413.

48. Barreiro E, Bustamante V, Cejudo P, Gáldiz JB, Gea J, de Lucas P, et al. Normativa SEPAR sobre disfunción muscular de los pacientes con enfermedad pulmonar obstructiva crónica. Arch Bronconeumol [Internet]. 2015 [citado 1 de octubre de 2016]; 51(8):384-95. Disponible en: https://medes.com/publication/101511

49. Maltais F, Decramer M, Casaburi R, Barreiro E, Burelle $Y$, Debigaré R, et al. An official American Thoracic Society/European Respiratory Society statement: update on limb muscle dysfunction in chronic obstructive pulmonary disease. Am J Respir Crit Care Med. 2014;189(9):e15-62.

50. Swallow EB, Reyes D, Hopkinson NS, Man WD-C, Porcher R, Cetti EJ, et al. Quadriceps strength predicts mortality in patients with moderate to severe chronic obstructive pulmonary disease. Thorax. 2007;62(2):115-20.

51. Bernard S, LeBlanc P, Whittom F, Carrier G, Jobin J, Belleau R, et al. Peripheral muscle weakness in patients with chronic obstructive pulmonary disease. Am J Respir Crit Care Med. 1998;158(2):629-34

52. Gosker HR, Zeegers MP, Wouters EFM, Schols AMWJ. Muscle fibre type shifting in the vastus lateralis of patients with COPD is associated with disease severity: a systematic review and meta-analysis. Thorax. 2007;62(11):944-9.

53. Kim HC, Mofarrahi M, Hussain SNA. Skeletal muscle dysfunction in patients with chronic obstructive pulmonary disease. Int J Chron Obstruct Pulmon Dis. 2008;3(4):637-58.

54. Jobin J, Maltais F, Doyon JF, LeBlanc P, Simard PM, Simard AA, et al. Chronic obstructive pulmonary disease: capillarity and fiber-type characteristics of skeletal muscle. J Cardpulm Rehabil. 1998;18(6):432-7.

55. Gosker HR, Hesselink MKC, Duimel H, Ward KA, Schols AMWJ. Reduced mitochondrial density in the vastus lateralis muscle of patients with COPD. Eur Respir J. 2007;30(1):73-9.

56. Naimi Al, Bourbeau J, Perrault H, Baril J, Wright-Paradis C, Rossi A, et al. Altered mitochondrial regulation in quadriceps muscles of patients with COPD. Clin Physiol Funct Imaging. 2011;31(2):124-31. 
57. Puente-Maestu L, Pérez-Parra J, Godoy R, Moreno N, Tejedor A, Torres A, et al. Abnormal transition pore kinetics and cytochrome $C$ release in muscle mitochondria of patients with chronic obstructive pulmonary disease. Am J Respir Cell Mol Biol. 2009;40(6):746-50.

58. Jakobsson $\mathrm{P}$, Jorfeldt $\mathrm{L}$, Henriksson J. Metabolic enzyme activity in the quadriceps femoris muscle in patients with severe chronic obstructive pulmonary disease. Am J Respir Crit Care Med. 1995;151(2 Pt 1):374-7.

59. Doucet M, Russell AP, Léger B, Debigaré R, Joanisse DR, Caron M-A, et al. Muscle atrophy and hypertrophy signaling in patients with chronic obstructive pulmonary disease. Am J Respir Crit Care Med. 2007;176(3):261-9.

60. Marin-Corral J, Minguella J, Ramírez-Sarmiento AL, Hussain SNA, Gea J, Barreiro E. Oxidised proteins and superoxide anion production in the diaphragm of severe COPD patients. Eur Respir J. 2009;33(6):1309-19.

61. Barreiro E, Gea J, Corominas JM, Hussain SNA. Nitric oxide synthases and protein oxidation in the quadriceps femoris of patients with chronic obstructive pulmonary disease. Am J Respir Cell Mol Biol. 2003;29(6):771-8.

62. Koechlin C, Maltais F, Saey D, Michaud A, LeBlanc P, Hayot M, et al. Hypoxaemia enhances peripheral muscle oxidative stress in chronic obstructive pulmonary disease. Thorax. 2005;60(10):834-41.

63. Couillard A, Prefaut C. From muscle disuse to myopathy in COPD: potential contribution of oxidative stress. Eur Respir J. 2005;26(4):703-19.

64. Schakman O, Gilson H, Kalista S, Thissen JP. Mechanisms of muscle atrophy induced by glucocorticoids. Horm Res. 2009;72 Suppl 1:36-41.

65. Man WD-C, Hopkinson NS, Harraf F, Nikoletou D, Polkey MI, Moxham J. Abdominal muscle and quadriceps strength in chronic obstructive pulmonary disease. Thorax. 2005;60(9):718-22.

66. Seymour JM, Ward K, Sidhu PS, Puthucheary Z, Steier J, Jolley CJ, et al. Ultrasound measurement of rectus femoris cross-sectional area and the relationship with quadriceps strength in COPD. Thorax. 2009;64(5):418-23.

67. Hopkinson NS, Tennant RC, Dayer MJ, Swallow EB, Hansel TT, Moxham J, et al. A prospective study of decline in fat free mass and skeletal muscle strength in chronic obstructive pulmonary disease. Respir Res. 2007;8:25.

68. van den Borst B, Slot IGM, Hellwig VACV, Vosse BAH, Kelders MCJM, Barreiro E, et al. Loss of quadriceps muscle oxidative phenotype and decreased endurance in patients with mild-to-moderate COPD. J Appl Physiol Bethesda Md 1985. 2013;114(9):1319-28.

69. Gosselink R, Troosters T, Decramer M. Peripheral muscle weakness contributes to exercise limitation in COPD. Am J Respir Crit Care Med. 1996;153(3):976-80.

70. Saey D, Debigare R, LeBlanc P, Mador MJ, Cote $\mathrm{CH}$, Jobin J, et al. Contractile leg fatigue after cycle exercise: a factor limiting exercise in patients with chronic obstructive pulmonary disease. Am J Respir Crit Care Med. 2003;168(4):425-30.

71. Casaburi R, Patessio A, Ioli F, Zanaboni S, Donner CF, Wasserman K. Reductions in exercise lactic acidosis and ventilation as a result of exercise training in patients with obstructive lung disease. Am Rev Respir Dis. 1991;143(1):9-18.

72. Spruit MA, Singh SJ, Garvey C, ZuWallack R, Nici L, Rochester C, et al. An official American Thoracic Society/European Respiratory Society statement: key concepts and advances in pulmonary rehabilitation. Am J Respir Crit Care Med. 2013;188(8):e13-64.

73. Lacasse Y, Goldstein R, Lasserson TJ, Martin S. Pulmonary rehabilitation for chronic obstructive pulmonary disease. Cochrane Database Syst Rev. 2006;(4):CD003793.

74. Puhan MA, Lareau SC. Evidence-based outcomes from pulmonary rehabilitation in the chronic obstructive pulmonary disease patient. Clin Chest Med. 2014;35(2):295-301. 
75. Garvey C, Bayles MP, Hamm LF, Hill K, Holland A, Limberg TM, et al. Pulmonary Rehabilitation Exercise Prescription in Chronic Obstructive Pulmonary Disease: Review of Selected Guidelines: AN OFFICIAL STATEMENT FROM THE AMERICAN ASSOCIATION OF CARDIOVASCULAR AND PULMONARY REHABILITATION. J Cardiopulm Rehabil Prev. 2016;36(2):75-83.

76. Laviolette L, Bourbeau J, Bernard S, Lacasse Y, Pepin V, Breton M-J, et al. Assessing the impact of pulmonary rehabilitation on functional status in COPD. Thorax [Internet]. 2008 [citado 1 de diciembre de 2016];63(2):115-21. Disponible en: http://thorax.bmj.com/content/63/2/115

77. Burtin C, Decramer M, Gosselink R, Janssens W, Troosters T. Rehabilitation and acute exacerbations. Eur Respir J. 2011;38(3):702-12.

78. Langer D, Hendriks E, Burtin C, Probst V, van der Schans C, Paterson W, et al. A clinical practice guideline for physiotherapists treating patients with chronic obstructive pulmonary disease based on a systematic review of available evidence. Clin Rehabil. 2009;23(5):445-62.

79. Criner GJ, Bourbeau J, Diekemper RL, Ouellette DR, Goodridge D, Hernandez P, et al. Executive summary: prevention of acute exacerbation of COPD: American College of Chest Physicians and Canadian Thoracic Society Guideline. Chest. 2015;147(4):883-93.

80. Puhan MA, Gimeno-Santos E, Cates CJ, Troosters T. Pulmonary rehabilitation following exacerbations of chronic obstructive pulmonary disease. Cochrane Database Syst Rev. 2016;12:CD005305.

81. Rous G, Rosa M, Díaz Lobato S, Rodríguez Trigo G, Morante Vélez F, San Miguel M, et al. Rehabilitación respiratoria. Arch Bronconeumol [Internet]. 2014 [citado 1 de octubre de 2016]; 50(8):332-44. Disponible en: http://www.archbronconeumol.org/es/rehabilitacion-respiratoria/articulo/S0300289614000878/

82. Peces-Barba G, Albert Barberà J, Agustí À, Casanova C, Casas A, Luis Izquierdo J, et al. Guía clínica SEPAR-ALAT de diagnóstico y tratamiento de la EPOC. Arch Bronconeumol [Internet]. 2008 [citado 1 de octubre de 2016]; 44(5):271-81. Disponible en: http://www.archbronconeumol.org/es/ guia-clinica-separ-alat-diagnostico-tratamiento/articulo/S0300289608704307/

83. Bolton CE, Bevan-Smith EF, Blakey JD, Crowe P, Elkin SL, Garrod R, et al. British Thoracic Society guideline on pulmonary rehabilitation in adults. Thorax. 2013;68 Suppl 2:ii1-30.

84. Man WD-C, Kemp P, Moxham J, Polkey MI. Exercise and muscle dysfunction in COPD: implications for pulmonary rehabilitation. Clin Sci Lond Engl 1979. 2009;117(8):281-91.

85. Vogiatzis I, Terzis G, Stratakos G, Cherouveim E, Athanasopoulos D, Spetsioti S, et al. Effect of pulmonary rehabilitation on peripheral muscle fiber remodeling in patients with COPD in GOLD stages II to IV. Chest. 2011;140(3):744-52.

86. Vogiatzis I, Simoes DCM, Stratakos G, Kourepini E, Terzis G, Manta P, et al. Effect of pulmonary rehabilitation on muscle remodelling in cachectic patients with COPD. Eur Respir J. 2010;36(2):301-10.

87. Ortega F, Toral J, Cejudo P, Villagomez R, Sánchez H, Castillo J, et al. Comparison of effects of strength and endurance training in patients with chronic obstructive pulmonary disease. Am J Respir Crit Care Med. 2002;166(5):669-74.

88. Garber CE, Blissmer B, Deschenes MR, Franklin BA, Lamonte MJ, Lee I-M, et al. American College of Sports Medicine position stand. Quantity and quality of exercise for developing and maintaining cardiorespiratory, musculoskeletal, and neuromotor fitness in apparently healthy adults: guidance for prescribing exercise. Med Sci Sports Exerc. 2011;43(7):1334-59.

89. Rochester CL, Vogiatzis I, Holland AE, Lareau SC, Marciniuk DD, Puhan MA, et al. An Official American Thoracic Society/European Respiratory Society Policy Statement: Enhancing Implementation, Use, and Delivery of Pulmonary Rehabilitation. Am J Respir Crit Care Med. 2015;192(11):1373-86.

90. Johnston K, Grimmer-Somers K. Pulmonary rehabilitation: overwhelming evidence but lost in translation? Physiother Can Physiother Can. 2010;62(4):368-73.

91. Güell MR, Cejudo P, Rodríguez-Trigo G, Gàldiz JB, Casolive V, Regueiro M, et al. Standards for quality care in respiratory rehabilitation in patients with chronic pulmonary disease. Quality Healthcare Committee. Spanish Society of Pneumology and Thoracic Surgery (SEPAR). Arch Bronconeumol. 2012;48(11):396-404. 
92. Güell-Rous MR, Diez-Betoret JL. Is respiratory rehabilitation really implemented today?. Clin Pulm Med. 2010;17(2):57-60.

93. Spruit MA, Pitta F, Garvey C, ZuWallack RL, Roberts CM, Collins EG, et al. Differences in content and organisational aspects of pulmonary rehabilitation programmes. Eur Respir J. 2014;43(5):1326-37.

94. Keating A, Lee A, Holland AE. What prevents people with chronic obstructive pulmonary disease from attending pulmonary rehabilitation? A systematic review. Chron Respir Dis. 2011;8(2):89-99.

95. Sabit R, Griffiths TL, Watkins AJ, Evans W, Bolton CE, Shale DJ, et al. Predictors of poor attendance at an outpatient pulmonary rehabilitation programme. Respir Med. 2008;102(6):819-24.

96. Fischer MJ, Scharloo M, Abbink JJ, Thijs-Van A, Rudolphus A, Snoei L, et al. Participation and drop-out in pulmonary rehabilitation: a qualitative analysis of the patient's perspective. Clin Rehabil. 2007;21(3):212-21.

97. Thorpe O, Johnston K, Kumar S. Barriers and enablers to physical activity participation in patients with COPD: a systematic review. J Cardiopulm Rehabil Prev. 2012;32(6):359-69.

98. Vieira DSR, Maltais F, Bourbeau J. Home-based pulmonary rehabilitation in chronic obstructive pulmonary disease patients. Curr Opin Pulm Med. 2010;16(2):134-43.

99. van Wetering CR, Hoogendoorn M, Mol SJM, Rutten-van Mölken MPMH, Schols AM. Short- and longterm efficacy of a community-based COPD management programme in less advanced COPD: a randomised controlled trial. Thorax. 2010;65(1):7-13.

100. Maltais F, Bourbeau J, Shapiro S, Lacasse Y, Perrault H, Baltzan M, et al. Effects of home-based pulmonary rehabilitation in patients with chronic obstructive pulmonary disease: a randomized trial. Ann Intern Med. 2008;149(12):869-78.

101. Resqueti V, Gorostiza A, Gáldiz JB, López de Santa María E, Casan Clarà P, Güell R. [Benefits of a homebased pulmonary rehabilitation program for patients with severe chronic obstructive pulmonary disease]. Arch Bronconeumol. 2007;43(11):599-604.

102. Liu W-T, Wang C-H, Lin H-C, Lin S-M, Lee K-Y, Lo Y-L, et al. Efficacy of a cell phone-based exercise programme for COPD. Eur Respir J. 2008;32(3):651-9.

103. Pradella CO, Belmonte GM, Maia MN, Delgado CS, Luise APT, Nascimento OA, et al. Home-Based Pulmonary Rehabilitation for Subjects With COPD: A Randomized Study. Respir Care. 2015;60(4):526-32.

104. de Sousa Pinto JM, Martín-Nogueras AM, Calvo-Arenillas Jl, Ramos-González J. Clinical benefits of home-based pulmonary rehabilitation in patients with chronic obstructive pulmonary disease. J Cardiopulm Rehabil Prev. 2014;34(5):355-9.

105. Pomidori L, Contoli M, Mandolesi G, Cogo A. A simple method for home exercise training in patients with chronic obstructive pulmonary disease: one-year study. J Cardiopulm Rehabil Prev. 2012;32(1):53-7.

106. Mendes de Oliveira JC, Studart Leitão Filho FS, Malosa Sampaio LM, Negrinho de Oliveira AC, Hirata $\mathrm{RP}$, Costa D, et al. Outpatient vs. home-based pulmonary rehabilitation in COPD: a randomized controlled trial. Multidiscip Respir Med. 2010;5(6):401-8.

107. Güell MR, de Lucas P, Gáldiz JB, Montemayor T, Rodríguez González-Moro JM, Gorostiza A, et al. [Home vs hospital-based pulmonary rehabilitation for patients with chronic obstructive pulmonary disease: a Spanish multicenter trial]. Arch Bronconeumol. 2008;44(10):512-8.

108. Kawagoshi A, Kiyokawa N, Sugawara K, Takahashi H, Sakata S, Satake M, et al. Effects of low-intensity exercise and home-based pulmonary rehabilitation with pedometer feedback on physical activity in elderly patients with chronic obstructive pulmonary disease. Respir Med. 2015;109(3):364-71.

109. Wang C-H, Chou P-C, Joa W-C, Chen L-F, Sheng T-F, Ho S-C, et al. Mobile-phone-based home exercise training program decreases systemic inflammation in COPD: a pilot study. BMC Pulm Med. 2014;14:142.

110. Ries AL, Kaplan RM, Myers R, Prewitt LM. Maintenance after pulmonary rehabilitation in chronic lung disease: a randomized trial. Am J Respir Crit Care Med. 2003;167(6):880-8. 
111. Berry MJ, Rejeski WJ, Adair NE, Ettinger WH, Zaccaro DJ, Sevick MA. A randomized, controlled trial comparing long-term and short-term exercise in patients with chronic obstructive pulmonary disease. J Cardpulm Rehabil. 2003;23(1):60-8.

112. Troosters T, Casaburi R, Gosselink R, Decramer M. Pulmonary rehabilitation in chronic obstructive pulmonary disease. Am J Respir Crit Care Med. 2005;172(1):19-38.

113. Troosters T, Gosselink R, Decramer M. Short- and long-term effects of outpatient rehabilitation in patients with chronic obstructive pulmonary disease: a randomized trial. Am J Med. 2000;109(3):207-12.

114. Ochmann U, Jörres RA, Nowak D. Long-term efficacy of pulmonary rehabilitation: a state-of-the-art review. J Cardiopulm Rehabil Prev. 2012;32(3):117-26.

115. ATS Committee on Proficiency Standards for Clinical Pulmonary Function Laboratories. ATS statement: guidelines for the six-minute walk test. Am J Respir Crit Care Med. 2002;166(1):111-7.

116. Rabinovich RA, Vilaró J, Roca J. [Evaluation exercise tolerance in COPD patients: the 6-minute walking test]. Arch Bronconeumol. 2004;40(2):80-5.

117. Spruit MA, Polkey MI, Celli B, Edwards LD, Watkins ML, Pinto-Plata V, et al. Predicting outcomes from 6-minute walk distance in chronic obstructive pulmonary disease. J Am Med Dir Assoc. 2012;13(3):291-7.

118. Casanova C, Cote CG, Marin JM, de Torres JP, Aguirre-Jaime A, Mendez R, et al. The 6-min walking distance: long-term follow up in patients with COPD. Eur Respir J. 2007;29(3):535-40.

119. Pinto-Plata VM, Cote C, Cabral H, Taylor J, Celli BR. The 6-min walk distance: change over time and value as a predictor of survival in severe COPD. Eur Respir J. 2004;23(1):28-33.

120. Oga T, Nishimura K, Tsukino M, Sato S, Hajiro T, Mishima M. Exercise capacity deterioration in patients with COPD: longitudinal evaluation over 5 years. Chest. 2005;128(1):62-9.

121. Ramon MA, Gimeno-Santos E, Ferrer J, Balcells E, Rodríguez E, de Batlle J, et al. Hospital admissions and exercise capacity decline in patients with COPD. Eur Respir J. 2014;43(4):1018-27.

122. Celli BR, Barnes PJ. Exacerbations of chronic obstructive pulmonary disease. Eur Respir J. 2007;29(6):1224-38.

123. Bu XN, Yang T, Thompson MA, Hutchinson AF, Irving LB. Changes in the BODE index, exacerbation duration and hospitalisation in a cohort of COPD patients. Singapore Med J. 2011;52(12):894-900.

124. Nici L. Can we make it last? Maintaining benefits achieved with pulmonary rehabilitation. Lung. 2007;185(5):241-2.

125. Celli BR, Decramer M, Wedzicha JA, Wilson KC, Agustí AA, Criner GJ, et al. An official American Thoracic Society/European Respiratory Society statement: research questions in COPD. Eur Respir Rev Off J Eur Respir Soc. 2015;24(136):159-72.

126. Busby AK, Reese RL, Simon SR. Pulmonary rehabilitation maintenance interventions: a systematic review. Am J Health Behav. 2014;38(3):321-30.

127. Beauchamp MK, Evans R, Janaudis-Ferreira T, Goldstein RS, Brooks D. Systematic review of supervised exercise programs after pulmonary rehabilitation in individuals with COPD. Chest. 2013;144(4):1124-33.

128. Ringbaek T, Brondum E, Martinez G, Thogersen J, Lange P. Long-term effects of 1-year maintenance training on physical functioning and health status in patients with COPD: A randomized controlled study. J Cardiopulm Rehabil Prev. 2010;30(1):47-52.

129. Spencer LM, Alison JA, McKeough ZJ. Maintaining benefits following pulmonary rehabilitation: a randomised controlled trial. Eur Respir J [Internet]. 2010 [citado 1 de enero de 2017];35(3):571-7. Disponible en: http://erj.ersjournals.com/content/35/3/571

130. Linneberg A, Rasmussen M, Buch TF, Wester A, Malm L, Fannikke G, et al. A randomised study of the effects of supplemental exercise sessions after a 7-week chronic obstructive pulmonary disease rehabilitation program. Clin Respir J. 2012;6(2):112-9. 
131. Wilson AM, Browne P, Olive S, Clark A, Galey P, Dix E, et al. The effects of maintenance schedules following pulmonary rehabilitation in patients with chronic obstructive pulmonary disease: a randomised controlled trial. BMJ Open. 2015;5(3):e005921.

132. Effing $T$, Zielhuis $G$, Kerstjens $H$, van der Valk $P$, van der Palen J. Community based physiotherapeutic exercise in COPD self-management: a randomised controlled trial. Respir Med. 2011;105(3):418-26.

133. du Moulin M, Taube K, Wegscheider K, Behnke M, van den Bussche H. Home-based exercise training as maintenance after outpatient pulmonary rehabilitation. Respir Int Rev Thorac Dis. 2009;77(2):139-45.

134. Heppner PS, Morgan C, Kaplan RM, Ries AL. Regular walking and long-term maintenance of outcomes after pulmonary rehabilitation. J Cardpulm Rehabil. 2006;26(1):44-53.

135. Steele BG, Belza B, Cain KC, Coppersmith J, Lakshminarayan S, Howard J, et al. A randomized clinical trial of an activity and exercise adherence intervention in chronic pulmonary disease. Arch Phys Med Rehabil. 2008;89(3):404-12.

136. Waterhouse JC, Walters SJ, Oluboyede Y, Lawson RA. A randomised $2 \times 2$ trial of community versus hospital pulmonary rehabilitation, followed by telephone or conventional follow-up. Health Technol Assess Winch Engl. 2010;14(6):i-v, vii-xi, 1-140.

137. Moullec G, Ninot G, Varray A, Desplan J, Hayot M, Prefaut C. An innovative maintenance follow-up program after a first inpatient pulmonary rehabilitation. Respir Med. 2008;102(4):556-66.

138. Barberan-Garcia A, Vogiatzis I, Solberg HS, Vilaró J, Rodríguez DA, Garåsen HM, et al. Effects and barriers to deployment of telehealth wellness programs for chronic patients across 3 European countries. Respir Med. 2014;108(4):628-37.

139. Norton K, Norton L, Sadgrove D. Position statement on physical activity and exercise intensity terminology. J Sci Med Sport. 2010;13(5):496-502.

140. Nelson ME, Rejeski WJ, Blair SN, Duncan PW, Judge JO, King AC, et al. Physical activity and public health in older adults: recommendation from the American College of Sports Medicine and the American Heart Association. Med Sci Sports Exerc. 2007;39(8):1435-45.

141. Schutz Y, Weinsier RL, Hunter GR. Assessment of free-living physical activity in humans: an overview of currently available and proposed new measures. Obes Res. 2001;9(6):368-79.

142. Tudor-Locke C, Bassett DR. How many steps/day are enough? Preliminary pedometer indices for public health. Sports Med AuckI NZ. 2004;34(1):1-8.

143. Hallal PC, Andersen LB, Bull FC, Guthold R, Haskell W, Ekelund U, et al. Global physical activity levels: surveillance progress, pitfalls, and prospects. Lancet Lond Engl. 2012;380(9838):247-57.

144. Bohannon RW. Number of pedometer-assessed steps taken per day by adults: a descriptive metaanalysis. Phys Ther. 2007;87(12):1642-50.

145. Ministerio de Sanidad, Servicios Sociales e Igualdad - Portal Estadístico del SNS - Sistema de Información Sanitaria: Portal Estadístico del SNS - Informe anual del Sistema Nacional de Salud. [citado 8 de octubre de 2016]; Disponible en: http://www.msssi.gob.es/estadEstudios/estadisticas/sisInfSanSNS/ tablasEstadisticas/InfAnSNS.htm

146. Owen N, Salmon J, Koohsari MJ, Turrell G, Giles-Corti B. Sedentary behaviour and health: mapping environmental and social contexts to underpin chronic disease prevention. Br J Sports Med. 2014;48(3):174-7.

147. Energy and protein requirements [Internet]. [citado 6 de octubre de 2016]. Disponible en: http://www. fao.org/docrep/003/aa040e/aa040e00.htm

148. Hospes G, Bossenbroek L, Ten Hacken NHT, van Hengel P, de Greef MHG. Enhancement of daily physical activity increases physical fitness of outclinic COPD patients: results of an exercise counseling program. Patient Educ Couns. 2009;75(2):274-8.

149. Casaburi R. Activity promotion: a paradigm shift for chronic obstructive pulmonary disease therapeutics. Proc Am Thorac Soc. 2011;8(4):334-7. 
150. Troosters T, van der Molen T, Polkey M, Rabinovich RA, Vogiatzis I, Weisman I, et al. Improving physical activity in COPD: towards a new paradigm. Respir Res. 2013;14:115.

151. Rabe KF, Hurd S, Anzueto A, Barnes PJ, Buist SA, Calverley P, et al. Global strategy for the diagnosis, management, and prevention of chronic obstructive pulmonary disease: GOLD executive summary. Am J Respir Crit Care Med. 2007;176(6):532-55.

152. Bossenbroek L, de Greef MHG, Wempe JB, Krijnen WP, Ten Hacken NHT. Daily physical activity in patients with chronic obstructive pulmonary disease: a systematic review. COPD. 2011;8(4):306-19.

153. Pitta F, Troosters T, Probst VS, Spruit MA, Decramer M, Gosselink R. Quantifying physical activity in daily life with questionnaires and motion sensors in COPD. Eur Respir J. 2006;27(5):1040-55.

154. Hill K, Gardiner PA, Cavalheri V, Jenkins SC, Healy GN. Physical activity and sedentary behaviour: applying lessons to chronic obstructive pulmonary disease. Intern Med J. 2015;45(5):474-82.

155. Watz H, Pitta F, Rochester CL, Garcia-Aymerich J, ZuWallack R, Troosters T, et al. An official European Respiratory Society statement on physical activity in COPD. Eur Respir J. 2014;44(6):1521-37.

156. Vorrink SNW, Kort HSM, Troosters T, Lammers J-WJ. Level of daily physical activity in individuals with COPD compared with healthy controls. Respir Res. 2011;12:33.

157. Troosters T, Sciurba F, Battaglia S, Langer D, Valluri SR, Martino L, et al. Physical inactivity in patients with COPD, a controlled multi-center pilot-study. Respir Med. 2010;104(7):1005-11.

158. Eliason G, Zakrisson A-B, Piehl-Aulin K, Hurtig-Wennlöf A. Physical activity patterns in patients in different stages of chronic obstructive pulmonary disease. COPD. 2011;8(5):369-74.

159. Pitta F, Troosters T, Spruit MA, Probst VS, Decramer M, Gosselink R. Characteristics of physical activities in daily life in chronic obstructive pulmonary disease. Am J Respir Crit Care Med. 2005;171(9):972-7.

160. Van Remoortel H, Hornikx M, Demeyer H, Langer D, Burtin C, Decramer M, et al. Daily physical activity in subjects with newly diagnosed COPD. Thorax. 2013;68(10):962-3.

161. Vitorasso R, Camillo CA, Cavalheri V, Aparecida Hernandes N, Cortez Verceze A, Sant'Anna T, et al. Is walking in daily life a moderate intensity activity in patients with chronic obstructive pulmonary disease? Eur J Phys Rehabil Med. 2012;48(4):587-92.

162. Pitta F, Troosters T, Probst VS, Lucas S, Decramer M, Gosselink R. Potential consequences for stable chronic obstructive pulmonary disease patients who do not get the recommended minimum daily amount of physical activity. J Bras Pneumol Publicacao Of Soc Bras Pneumol E Tisilogia. 2006;32(4):301-8.

163. Waschki B, Spruit MA, Watz H, Albert PS, Shrikrishna D, Groenen M, et al. Physical activity monitoring in COPD: compliance and associations with clinical characteristics in a multicenter study. Respir Med. 2012;106(4):522-30.

164. McGlone S, Venn A, Walters EH, Wood-Baker R. Physical activity, spirometry and quality-of-life in chronic obstructive pulmonary disease. COPD. 2006;3(2):83-8.

165. Tudor-Locke C, Washington TL, Hart TL. Expected values for steps/day in special populations. Prev Med. 2009;49(1):3-11.

166. Arne M, Janson C, Janson S, Boman G, Lindqvist U, Berne C, et al. Physical activity and quality of life in subjects with chronic disease: chronic obstructive pulmonary disease compared with rheumatoid arthritis and diabetes mellitus. Scand J Prim Health Care. 2009;27(3):141-7.

167. Gouzi F, Préfaut C, Abdellaoui A, Vuillemin A, Molinari N, Ninot G, et al. Evidence of an early physical activity reduction in chronic obstructive pulmonary disease patients. Arch Phys Med Rehabil. 2011;92(10):1611-1617.e2.

168. Moy ML, Danilack VA, Weston NA, Garshick E. Daily step counts in a US cohort with COPD. Respir Med. 2012;106(7):962-9. 
169. Altenburg WA, Bossenbroek L, de Greef MHG, Kerstjens HAM, ten Hacken NHT, Wempe JB. Functional and psychological variables both affect daily physical activity in COPD: a structural equations model. Respir Med. 2013;107(11):1740-7.

170. Moy ML, Teylan M, Weston NA, Gagnon DR, Garshick E. Daily step count predicts acute exacerbations in a US cohort with COPD. PloS One. 2013;8(4):e60400.

171. Hartman JE, Boezen HM, de Greef MH, Ten Hacken NH. Physical and psychosocial factors associated with physical activity in patients with chronic obstructive pulmonary disease. Arch Phys Med Rehabil. 2013;94(12):2396-2402.e7.

172. Zwerink M, van der Palen J, van der Valk P, Brusse-Keizer M, Effing T. Relationship between daily physical activity and exercise capacity in patients with COPD. Respir Med. 2013;107(2):242-8.

173. Waschki B, Kirsten AM, Holz O, Mueller K-C, Schaper M, Sack A-L, et al. Disease Progression and Changes in Physical Activity in Patients with Chronic Obstructive Pulmonary Disease. Am J Respir Crit Care Med. 2015;192(3):295-306.

174. Nyssen SM, Santos JG dos, Barusso MS, Oliveira Jr AD de, Lorenzo VAPD, Jamami M. Levels of physical activity and predictors of mortality in COPD. J Bras Pneumol Publicacao Of Soc Bras Pneumol E Tisilogia. 2013;39(6):659-66.

175. Mador MJ, Patel AN, Nadler J. Effects of pulmonary rehabilitation on activity levels in patients with chronic obstructive pulmonary disease. J Cardiopulm Rehabil Prev. 2011;31(1):52-9.

176. Garcia-Aymerich J, Félez MA, Escarrabill J, Marrades RM, Morera J, Elosua R, et al. Physical activity and its determinants in severe chronic obstructive pulmonary disease. Med Sci Sports Exerc. 2004;36(10):1667-73.

177. Waschki B, Kirsten A, Holz O, Müller K-C, Meyer T, Watz H, et al. Physical activity is the strongest predictor of all-cause mortality in patients with COPD: a prospective cohort study. Chest. 2011;140(2):331-42.

178. Garcia-Rio F, Rojo B, Casitas R, Lores V, Madero R, Romero D, et al. Prognostic value of the objective measurement of daily physical activity in patients with COPD. Chest. 2012;142(2):338-46.

179. Garcia-Aymerich J, Serra I, Gómez FP, Farrero E, Balcells E, Rodríguez DA, et al. Physical activity and clinical and functional status in COPD. Chest. 2009;136(1):62-70.

180. Pitta F, Takaki MY, Oliveira NH de, Sant'anna TJP, Fontana AD, Kovelis D, et al. Relationship between pulmonary function and physical activity in daily life in patients with COPD. Respir Med. 2008;102(8):1203-7.

181. Garcia-Rio F, Lores V, Mediano O, Rojo B, Hernanz A, López-Collazo E, et al. Daily physical activity in patients with chronic obstructive pulmonary disease is mainly associated with dynamic hyperinflation. Am J Respir Crit Care Med. 2009;180(6):506-12.

182. Gimeno-Santos E, Frei A, Steurer-Stey C, de Batlle J, Rabinovich RA, Raste Y, et al. Determinants and outcomes of physical activity in patients with COPD: a systematic review. Thorax. agosto de 2014;69(8):731-9.

183. Watz H, Waschki B, Meyer T, Magnussen H. Physical activity in patients with COPD. Eur Respir J. 2009;33(2):262-72.

184. Jehn M, Schmidt-Trucksäss A, Meyer A, Schindler C, Tamm M, Stolz D. Association of daily physical activity volume and intensity with COPD severity. Respir Med. 2011;105(12):1846-52.

185. Watz H, Waschki B, Boehme C, Claussen M, Meyer T, Magnussen H. Extrapulmonary effects of chronic obstructive pulmonary disease on physical activity: a cross-sectional study. Am J Respir Crit Care Med. 2008;177(7):743-51.

186. Durheim MT, Smith PJ, Babyak MA, Mabe SK, Martinu T, Welty-Wolf KE, et al. Six-minute-walk distance and accelerometry predict outcomes in chronic obstructive pulmonary disease independent of Global Initiative for Chronic Obstructive Lung Disease 2011 Group. Ann Am Thorac Soc. 2015;12(3):349-56.

187. Mantoani LC, Hernandes NA, Guimarães MM, Vitorasso RL, Probst VS, Pitta F. Does the BODE index reflect the level of physical activity in daily life in patients with COPD? Rev Bras Fisioter Sao Carlos Sao Paulo Braz. 2011;15(2):131-7. 
188. Moy ML, Teylan M, Danilack VA, Gagnon DR, Garshick E. An index of daily step count and systemic inflammation predicts clinical outcomes in chronic obstructive pulmonary disease. Ann Am Thorac Soc. 2014;11(2):149-57.

189. Barriga S, Rodrigues F, Bárbara C. Factors that influence physical activity in the daily life of male patients with chronic obstructive pulmonary disease. Rev Port Pneumol. 2014;20(3):131-7.

190. Hill K, Dolmage TE, Woon L, Coutts D, Goldstein R, Brooks D. Defining the relationship between average daily energy expenditure and field-based walking tests and aerobic reserve in COPD. Chest. 2012;141(2):406-12.

191. Polkey MI, Moxham J. Attacking the disease spiral in chronic obstructive pulmonary disease. Clin Med Lond Engl. 2006;6(2):190-6.

192. Pitta F, Troosters T, Probst VS, Spruit MA, Decramer M, Gosselink R. Physical activity and hospitalization for exacerbation of COPD. Chest. 2006;129(3):536-44.

193. Alahmari AD, Patel ARC, Kowlessar BS, Mackay AJ, Singh R, Wedzicha JA, et al. Daily activity during stability and exacerbation of chronic obstructive pulmonary disease. BMC Pulm Med. 2014;14:98.

194. Hopkinson NS, Polkey MI. Does physical inactivity cause chronic obstructive pulmonary disease? Clin Sci Lond Engl 1979. 2010;118(9):565-72.

195. Marín Royo M, Pellicer Císcar C, González Villaescusa C, Bueso Fabra MJ, Aguar Benito C, Andreu Rodríguez AL, et al. [Physical activity and its relationship with the state of health of stable COPD patients]. Arch Bronconeumol. 2011;47(7):335-42.

196. Watz H, Waschki B, Kirsten A, Müller K-C, Kretschmar G, Meyer T, et al. The metabolic syndrome in patients with chronic bronchitis and COPD: frequency and associated consequences for systemic inflammation and physical inactivity. Chest. 2009;136(4):1039-46.

197. Esteban C, Arostegui I, Aburto M, Moraza J, Quintana JM, Aizpiri S, et al. Influence of changes in physical activity on frequency of hospitalization in chronic obstructive pulmonary disease. Respirol Carlton Vic. 2014;19(3):330-8.

198. Sievi NA, Senn O, Brack T, Brutsche MH, Frey M, Irani S, et al. Impact of comorbidities on physical activity in COPD. Respirol Carlton Vic. 2015;20(3):413-8.

199. Gimeno-Santos E, Raste Y, Demeyer H, Louvaris Z, de Jong C, Rabinovich RA, et al. The PROactive instruments to measure physical activity in patients with chronic obstructive pulmonary disease. Eur Respir J. 2015;46(4):988-1000.

200. Montemayor T, Llorca E, López JL, Marcos PJ, Marcos P, Marín A, Marín JM, martínez C, Mayoral S, Muñoz L, Ortega F. EPOC: consenso de expertos EXPERT 10. Ejercicio físico/calidad de vida. Monog Arch Bronconeumol. 2014;1(1):460-9.

201. Nguyen HQ, Chu L, Amy Liu I-L, Lee JS, Suh D, Korotzer B, et al. Associations between physical activity and 30-day readmission risk in chronic obstructive pulmonary disease. Ann Am Thorac Soc. 2014;11(5):695-705.

202. Benzo RP, Chang C-CH, Farrell MH, Kaplan R, Ries A, Martinez FJ, et al. Physical activity, health status and risk of hospitalization in patients with severe chronic obstructive pulmonary disease. Respir Int Rev Thorac Dis. 2010;80(1):10-8.

203. Garcia-Aymerich J, Lange P, Benet M, Schnohr P, Antó JM. Regular physical activity reduces hospital admission and mortality in chronic obstructive pulmonary disease: a population based cohort study. Thorax. 2006;61(9):772-8.

204. Garcia-Aymerich J, Farrero E, Félez MA, Izquierdo J, Marrades RM, Antó JM, et al. Risk factors of readmission to hospital for a COPD exacerbation: a prospective study. Thorax. 2003;58(2):100-5.

205. Vaes AW, Garcia-Aymerich J, Marott JL, Benet M, Groenen MTJ, Schnohr P, et al. Changes in physical activity and all-cause mortality in COPD. Eur Respir J. 2014;44(5):1199-209. 
206. Jehn M, Schindler C, Meyer A, Tamm M, Schmidt-Trucksäss A, Stolz D. Daily walking intensity as a predictor of quality of life in patients with chronic obstructive pulmonary disease. Med Sci Sports Exerc. 2012;44(7):1212-8.

207. Esteban C, Quintana JM, Aburto M, Moraza J, Egurrola M, Pérez-Izquierdo J, et al. Impact of changes in physical activity on health-related quality of life among patients with COPD. Eur Respir J. 2010;36(2):292-300.

208. Troosters T, Gosselink R, Janssens W, Decramer M. Exercise training and pulmonary rehabilitation: new insights and remaining challenges. Eur Respir Rev Off J Eur Respir Soc. 2010;19(115):24-9.

209. Cindy Ng LW, Mackney J, Jenkins S, Hill K. Does exercise training change physical activity in people with COPD? A systematic review and meta-analysis. Chron Respir Dis. 2012;9(1):17-26.

210. Burtin C, Langer D, van Remoortel H, Demeyer H, Gosselink R, Decramer M, et al. Physical Activity Counselling during Pulmonary Rehabilitation in Patients with COPD: A Randomised Controlled Trial. PLoS ONE [Internet]. 2015 [citado 7 de abril de 2017];10(12). Disponible en: http://www.ncbi.nlm.nih.gov/pmc/ articles/PMC4689370/

211. Williams V, Bruton A, Ellis-Hill C, McPherson K. The effect of pulmonary rehabilitation on perceptions of breathlessness and activity in COPD patients: a qualitative study. Prim Care Respir J J Gen Pract Airw Group. 2010;19(1):45-51.

212. Mantoani LC, Rubio N, McKinstry B, MacNee W, Rabinovich RA. Interventions to modify physical activity in patients with COPD: a systematic review. Eur Respir J. 2016;48(1):69-81.

213. Spruit MA, Pitta F, McAuley E, ZuWallack RL, Nici L. Pulmonary Rehabilitation and Physical Activity in Patients with Chronic Obstructive Pulmonary Disease. Am J Respir Crit Care Med. 2015;192(8):924-33.

214. Soicher JE, Mayo NE, Gauvin L, Hanley JA, Bernard S, Maltais F, et al. Trajectories of endurance activity following pulmonary rehabilitation in COPD patients. Eur Respir J [Internet]. 2012 [citado 1 de enero de 2017];39(2): 272-8. Disponible en: http://erj.ersjournals.com/content/39/2/272

215. Pitta F, Troosters T, Probst VS, Langer D, Decramer M, Gosselink R. Are patients with COPD more active after pulmonary rehabilitation? Chest. 2008;134(2):273-80.

216. Pleguezuelos E, Pérez ME, Guirao L, Samitier B, Ortega P, Vila X, et al. Improving physical activity in patients with COPD with urban walking circuits. Respir Med. 2013;107(12):1948-56.

217. Donesky-Cuenco D, Janson S, Neuhaus J, Neilands TB, Carrieri-Kohlman V. Adherence to a homewalking prescription in patients with chronic obstructive pulmonary disease. Heart Lung J Crit Care. 2007;36(5):348-63.

218. Zwerink M, van der Palen J, Kerstjens HAM, van der Valk P, Brusse-Keizer M, Zielhuis G, et al. A community-based exercise programme in COPD self-management: two years follow-up of the COPE-II study. Respir Med. 2014;108(10):1481-90.

219. Wilson JJ, O'Neill B, Collins EG, Bradley J. Interventions to Increase Physical Activity in Patients with COPD: A Comprehensive Review. COPD. 2015;12(3):332-43.

220. Heath GW, Parra DC, Sarmiento OL, Andersen LB, Owen N, Goenka S, et al. Evidence-based intervention in physical activity: lessons from around the world. Lancet Lond Engl. 2012;380(9838):272-81.

221. Leidy NK, Kimel M, Ajagbe L, Kim K, Hamilton A, Becker K. Designing trials of behavioral interventions to increase physical activity in patients with COPD: Insights from the chronic disease literature. Respir Med [Internet]. 2014 [citado 15 de octubre de 2016];108(3):472-81. Disponible en: / article/S0954-6111(13)00452-6/abstract

222. Vaes AW, Cheung A, Atakhorrami M, Groenen MTJ, Amft O, Franssen FME, et al. Effect of «activity monitor-based» counseling on physical activity and health-related outcomes in patients with chronic diseases: A systematic review and meta-analysis. Ann Med. 2013;45(5-6):397-412.

223. Turner LJ, Houchen L, Williams J, Singh SJ. Reliability of pedometers to measure step counts in patients with chronic respiratory disease. J Cardiopulm Rehabil Prev. 2012;32(5):284-91. 
224. Ewald B, Duke J, Thakkinstian A, Attia J, Smith W. Physical activity of older Australians measured by pedometry. Australas J Ageing. 2009;28(3):127-33.

225. Bravata DM, Smith-Spangler C, Sundaram V, Gienger AL, Lin N, Lewis $R$, et al. Using pedometers to increase physical activity and improve health: a systematic review. JAMA. 2 2007;298(19):2296-304.

226. Greef KPD, Deforche BI, Ruige JB, Bouckaert JJ, Tudor-Locke CE, Kaufman J-M, et al. The effects of a pedometer-based behavioral modification program with telephone support on physical activity and sedentary behavior in type 2 diabetes patients. Patient Educ Couns [Internet]. 2011 [citado 14 de junio de 2016]; 84(2):275-9. Disponible en: http://www.pec-journal.com/article/S0738399110004118/abstract

227. Snyder A, Colvin B, Gammack JK. Pedometer use increases daily steps and functional status in older adults. J Am Med Dir Assoc. 2011;12(8):590-4.

228. Velthuis MJ, May AM, Koppejan-Rensenbrink RA, Gijsen BCM, van Breda E, de Wit GA, et al. Physical Activity during Cancer Treatment (PACT) Study: design of a randomised clinical trial. BMC Cancer. 2010;10:272.

229. Furber S, Butler L, Phongsavan P, Mark A, Bauman A. Randomised controlled trial of a pedometerbased telephone intervention to increase physical activity among cardiac patients not attending cardiac rehabilitation. Patient Educ Couns [Internet]. 2010 [citado 14 de junio de 2016]; 80(2):212-8. Disponible en: http://www.pec-journal.com/article/S0738399109005898/abstract

230. Chan CB, Tudor-Locke C. Real-world evaluation of a community-based pedometer intervention. J Phys Act Health. 2008;5(5):648-64.

231. Bjørgaas MR, Vik JT, Stølen T, Lydersen S, Grill V. Regular use of pedometer does not enhance beneficial outcomes in a physical activity intervention study in type 2 diabetes mellitus. Metabolism. 2008;57(5):605-11.

232. Murphy TP, Hirsch AT, Ricotta JJ, Cutlip DE, Mohler E, Regensteiner JG, et al. The Claudication: Exercise Vs. Endoluminal Revascularization (CLEVER) study: rationale and methods. J Vasc Surg. 2008;47(6):1356-63.

233. Crowther RG, Spinks WL, Leicht AS, Sangla K, Quigley F, Golledge J. Effects of a long-term exercise program on lower limb mobility, physiological responses, walking performance, and physical activity levels in patients with peripheral arterial disease. J Vasc Surg. 2008;47(2):303-9.

234. Locke EA, Lathman GP, Smith KJ. A theory of goal stting and task performance. Prentice Hall: Englewood ClifsN.J.; 1990.

235. Hartman JE, Boezen HM, Zuidema MJ, de Greef MHG, Ten Hacken NHT. Physical activity recommendations in patients with chronic obstructive pulmonary disease. Respir Int Rev Thorac Dis. 2014;88(2):92-100.

236. Altenburg WA, ten Hacken NHT, Bossenbroek L, Kerstjens HAM, de Greef MHG, Wempe JB. Shortand long-term effects of a physical activity counselling programme in COPD: a randomized controlled trial. Respir Med. 2015;109(1):112-21.

237. Moy ML, Collins RJ, Martinez CH, Kadri R, Roman P, Holleman RG, et al. An Internet-Mediated Pedometer-Based Program Improves Health-Related Quality-of-Life Domains and Daily Step Counts in COPD: A Randomized Controlled Trial. Chest. 2015;148(1):128-37.

238. Moy ML, Weston NA, Wilson EJ, Hess ML, Richardson CR. A pilot study of an Internet walking program and pedometer in COPD. Respir Med. 2012;106(9):1342-50.

239. Moy ML, Janney AW, Nguyen HQ, Matthess KR, Cohen M, Garshick E, et al. Use of pedometer and Internet-mediated walking program in patients with chronic obstructive pulmonary disease. J Rehabil Res Dev. 2010;47(5):485-96.

240. Nguyen HQ, Gill DP, Wolpin S, Steele BG, Benditt JO. Pilot study of a cell phone-based exercise persistence intervention post-rehabilitation for COPD. Int J Chron Obstruct Pulmon Dis. 2009;4:301-13.

241. de Blok BMJ, de Greef MHG, ten Hacken NHT, Sprenger SR, Postema K, Wempe JB. The effects of a lifestyle physical activity counseling program with feedback of a pedometer during pulmonary rehabilitation in patients with COPD: a pilot study. Patient Educ Couns. 2006;61(1):48-55. 
242. Mendoza L, Horta P, Espinoza J, Aguilera M, Balmaceda N, Castro A, et al. Pedometers to enhance physical activity in COPD: a randomised controlled trial. Eur Respir J [Internet]. 2015 [citado 19 de marzo de 2017];45(2):347-54. Disponible en: http://erj.ersjournals.com/content/45/2/347

243. Kocks JWH, Asijee GM, Tsiligianni IG, Kerstjens HAM, van der Molen T. Functional status measurement in COPD: a review of available methods and their feasibility in primary care. Prim Care Respir J J Gen Pract Airw Group. 2011;20(3):269-75.

244. Depew ZS, Novotny PJ, Benzo RP. How many steps are enough to avoid severe physical inactivity in patients with chronic obstructive pulmonary disease? Respirol Carlton Vic. 2012;17(6):1026-7.

245. Furlanetto KC, Bisca GW, Oldemberg N, Sant'anna TJ, Morakami FK, Camillo CA, et al. Step counting and energy expenditure estimation in patients with chronic obstructive pulmonary disease and healthy elderly: accuracy of 2 motion sensors. Arch Phys Med Rehabil. 2010;91(2):261-7.

246. Danilack VA, Okunbor O, Richardson CR, Teylan M, Moy ML. Performance of a pedometer to measure physical activity in a U.S. cohort with chronic obstructive pulmonary disease. J Rehabil Res Dev. 2015;52(3):333-42.

247. Puente-Maestu L, Sánz ML, Sánz P, Cubillo JM, Mayol J, Casaburi R. Comparison of effects of supervised versus self-monitored training programmes in patients with chronic obstructive pulmonary disease. Eur Respir J. 2000;15(3):517-25.

248. Cruz J, Brooks D, Marques A. Walk2Bactive: A randomised controlled trial of a physical activity-focused behavioural intervention beyond pulmonary rehabilitation in chronic obstructive pulmonary disease. Chron Respir Dis. 2016;13(1):57-66.

249. Holland AE, Spruit MA, Troosters T, Puhan MA, Pepin V, Saey D, et al. An official European Respiratory Society/American Thoracic Society technical standard: field walking tests in chronic respiratory disease. Eur Respir J. 2014;44(6):1428-46.

250. Puente L, Martín JM, Burgos F, Cobos N, Casanova C, Cueto A, De Miguel J, Villena V, Zamarrón C. Manual SEPAR de Procedimientos. Procedimientos de evaluación de la función pulmonar. Barcelona: LUZAN 5; 2002. 4-15 p.

251. García-Río F, Calle M, Burgos F, Casan P, Del Campo F, Gáldiz JR, Gon'zalez-Mangado F, Ortega F, Puente L. Normativas SEPAR. Normativa sobre la espirometría (revisión 2013). Barcelona: Respira; 2013.

252. Seemungal TA, Donaldson GC, Bhowmik A, Jeffries DJ, Wedzicha JA. Time course and recovery of exacerbations in patients with chronic obstructive pulmonary disease. Am J Respir Crit Care Med. 2000;161(5):1608-13.

253. Borg GA. Psychophysical bases of perceived exertion. Med Sci Sports Exerc. 1982;14(5):377-81.

254. Task group on surveillance for respiratory hazards in the occupational setting, Brooks SM (Chairman). Survei llance for respiratory hazards. ATS News. 1982;12-6.

255. Barreiro E, Burgos F, Calaf N, Casan P, Compte L, Clar RD, Fernández MJ, Gáldiz JB, López E. Manual SEPAR de Procedimientos. Procedimientos de evaluación de la función pulmonar II. Barcelona: Permayer; 2004. 134-145 p.

256. Morales P, Sanchis J, Cordero PJ, Díez JL. [Maximum static respiratory pressures in adults. The reference values for a Mediterranean Caucasian population]. Arch Bronconeumol. 1997;33(5):213-9.

257. Giménez M. La ventilation dirigée au cours des insuffisances respiratories croniques. 1968.

258. Thoman RL, Stoker GL, Ross JC. The efficacy of pursed-lips breathing in patients with chronic obstructive pulmonary disease. Am Rev Respir Dis. 1966;93(1):100-6.

259. Spahija J, de Marchie M, Grassino A. Effects of imposed pursed-lips breathing on respiratory mechanics and dyspnea at rest and during exercise in COPD. Chest. 2005;128(2):640-50.

260. Bott J, Blumenthal S, Buxton M, Ellum S, Falconer C, Garrod R, et al. Guidelines for the physiotherapy management of the adult, medical, spontaneously breathing patient. Thorax. 2009;64 Suppl 1:i1-51. 
261. Holland AE, Hill CJ, Jones AY, McDonald CF. Breathing exercises for chronic obstructive pulmonary disease. Cochrane Database Syst Rev. 2012;10:CD008250.

262. Postiaux G, Lens E, Alsteens G, Portelange P. Efficacité de l'expiration lente totale glotte ouverte en decubitus lateral (ELTGOL): sur la toilette en périphérie de l'arble trachéobronchique. Ann Kinésithérapie. 1990;97-9.

263. Pryor JA, Webber BA. An evaluation of the forced expiration technique as an adjunct to postural drainage. Physiotherapy. 1979;65(10):304-7.

264. Hristara-Papadopoulou A, Tsanakas J, Diomou G, Papadopoulou O. Current devices of respiratory physiotherapy. Hippokratia. 2008;12(4):211-20.

265. Myers TR. Positive expiratory pressure and oscillatory positive expiratory pressure therapies. Respir Care. 2007;52(10):1308-1326; discussion 1327.

266. Manual SEPAR de Procedimientos 27. Técnicas manuales e instrumentales para el drenaje de secreciones [Internet]. Issuu. [citado 7 de octubre de 2016]. Disponible en: https://issuu.com/separ/docs/manual_27/23

267. Giraldo H. EPOC. Diagnóstico y tratamiento integral. Bogotá: Panamericana; 2008. 282-291 p.

268. Singh SJ, Morgan MD, Scott S, Walters D, Hardman AE. Development of a shuttle walking test of disability in patients with chronic airways obstruction. Thorax. 1992;47(12):1019-24.

269. Singh SJ, Morgan MD, Hardman AE, Rowe C, Bardsley PA. Comparison of oxygen uptake during a conventional treadmill test and the shuttle walking test in chronic airflow limitation. Eur Respir J. 1994;7(11):2016-20.

270. Arnardóttir RH, Emtner M, Hedenström H, Larsson K, Boman G. Peak exercise capacity estimated from incremental shuttle walking test in patients with COPD: a methodological study. Respir Res. 2006;7:127.

271. Viel E. Facilitación Neuromuscular Propioceptiva. Principios y técnicas. Buenos Aires: Panamericana; 1984. 82-91 p.

272. OMS | 10 datos sobre la obesidad [Internet]. [citado 1 de enero de 2017]. Disponible en: http://www. who.int/features/factfiles/obesity/facts/es/

273. Holland $A E$, Nici L. The return of the minimum clinically important difference for 6-minute-walk distance in chronic obstructive pulmonary disease. Am J Respir Crit Care Med. 2013;187(4):335-6.

274. Puhan MA, Chandra D, Mosenifar Z, Ries A, Make B, Hansel NN, et al. The minimal important difference of exercise tests in severe COPD. Eur Respir J. 2011;37(4):784-90.

275. Puhan MA, Mador MJ, Held U, Goldstein R, Guyatt GH, Schünemann HJ. Interpretation of treatment changes in 6-minute walk distance in patients with COPD. Eur Respir J. 2008;32(3):637-43.

276. Polkey MI, Spruit MA, Edwards LD, Watkins ML, Pinto-Plata V, Vestbo J, et al. Six-minute-walk test in chronic obstructive pulmonary disease: minimal clinically important difference for death or hospitalization. Am J Respir Crit Care Med. 2013;187(4):382-6.

277. Pepin V, Laviolette L, Brouillard C, Sewell L, Singh SJ, Revill SM, et al. Significance of changes in endurance shuttle walking performance. Thorax. 2011;66(2):115-20.

278. Charlson M, Szatrowski TP, Peterson J, Gold J. Validation of a combined comorbidity index. J Clin Epidemiol. 1994;47(11):1245-51.

279. Revill SM, Morgan MD, Singh SJ, Williams J, Hardman AE. The endurance shuttle walk: a new field test for the assessment of endurance capacity in chronic obstructive pulmonary disease. Thorax. 1999;54(3):213-22.

280. ERS Task Force, Palange P, Ward SA, Carlsen K-H, Casaburi R, Gallagher CG, et al. Recommendations on the use of exercise testing in clinical practice. Eur Respir J. 2007;29(1):185-209.

281. Borel B, Pepin V, Mahler DA, Nadreau É, Maltais F. Prospective validation of the endurance shuttle walking test in the context of bronchodilation in COPD. Eur Respir J. 2014;44(5):1166-76. 
282. Rider BC, Bassett DR, Thompson DL, Steeves EA, Raynor H. Monitoring capabilities of the Omron HJ720ITC pedometer. Phys Sportsmed. 2014;42(1):24-9.

283. Holbrook EA, Barreira TV, Kang M. Validity and reliability of Omron pedometers for prescribed and self-paced walking. Med Sci Sports Exerc. 2009;41(3):670-4.

284. Dallas MI, McCusker C, Haggerty MC, Rochester CL, Zuwallack R, Northeast Pulmonary Rehabilitation Consortium. Using pedometers to monitor walking activity in outcome assessment for pulmonary rehabilitation. Chron Respir Dis. 2009;6(4):217-24.

285. Vilaró J, Gimeno E, Sánchez Férez N, Hernando C, Díaz I, Ferrerc M, et al. [Daily living activity in chronic obstructive pulmonary disease: validation of the Spanish version and comparative analysis of 2 questionnaires]. Med Clin (Barc). 2007;129(9):326-32.

286. Jones PW, Quirk FH, Baveystock CM. The St George's Respiratory Questionnaire. Respir Med. 1991;85 Suppl B:25-31; discussion 33-37.

287. Güell R, Casan P, Sangenís M, Morante F, Belda J, Guyatt GH. Quality of life in patients with chronic respiratory disease: the Spanish version of the Chronic Respiratory Questionnaire (CRQ). Eur Respir J. 1998;11(1):55-60.

288. Jones PW. Interpreting thresholds for a clinically significant change in health status in asthma and COPD. Eur Respir J. 2002;19(3):398-404.

289. Consenso SEPAR-ALAT sobre terapia inhalada. issuu [Internet]. [citado 29 de diciembre de 2016]; Disponible en: https://issuu.com/separ/docs/consenso_separ-alat_sobre_terapia_i/7?reader3=1

290. Casas A, Vilaro J, Rabinovich R, Mayer A, Barberà JA, Rodriguez-Roisin R, et al. Encouraged 6-min walking test indicates maximum sustainable exercise in COPD patients. Chest. 2005;128(1):55-61.

291. Casaburi R, Porszasz J, Burns MR, Carithers ER, Chang RS, Cooper CB. Physiologic benefits of exercise training in rehabilitation of patients with severe chronic obstructive pulmonary disease. Am J Respir Crit Care Med. 1997;155(5):1541-51.

292. Rubí M, Renom F, Ramis F, Medinas M, Centeno MJ, Górriz M, et al. Effectiveness of pulmonary rehabilitation in reducing health resources use in chronic obstructive pulmonary disease. Arch Phys Med Rehabil. 2010;91(3):364-8.

293. Gosselink R, De Vos J, van den Heuvel SP, Segers J, Decramer M, Kwakkel G. Impact of inspiratory muscle training in patients with COPD: what is the evidence? Eur Respir J. 2011;37(2):416-25.

294. Shoemaker MJ, Donker S, Lapoe A. Inspiratory muscle training in patients with chronic obstructive pulmonary disease: the state of the evidence. Cardiopulm Phys Ther J. 2009;20(3):5-15.

295. Geddes EL, O'Brien K, Reid WD, Brooks D, Crowe J. Inspiratory muscle training in adults with chronic obstructive pulmonary disease: an update of a systematic review. Respir Med. 2008;102(12):1715-29.

296. Neves LF, Reis MH, Plentz RDM, Matte DL, Coronel CC, Sbruzzi G. Expiratory and expiratory plus inspiratory muscle training improves respiratory muscle strength in subjects with COPD: systematic review. Respir Care. 2014;59(9):1381-8.

297. Weiner P, Magadle R, Beckerman M, Weiner M, Berar-Yanay N. Maintenance of inspiratory muscle training in COPD patients: one year follow-up. Eur Respir J. 2004;23(1):61-5.

298. Dias FD, Sampaio LMM, da Silva GA, Gomes ÉLFD, do Nascimento ESP, Alves VLS, et al. Home-based pulmonary rehabilitation in patients with chronic obstructive pulmonary disease: a randomized clinical trial. Int J Chron Obstruct Pulmon Dis. 2013;8:537-44.

299. Wootton SL, Ng LWC, McKeough ZJ, Jenkins S, Hill K, Eastwood PR, et al. Ground-based walking training improves quality of life and exercise capacity in COPD. Eur Respir J. 2014;44(4):885-94.

300. Alonso CJLI. EPOC: consenso de expertos EXPERT 10. Disnea/síntomas. Monogr Arch Bronconeumol [Internet]. 2014 [citado 19 de abril de 2016]; 1(1). Disponible en: http://separcontenidos.es/revista/index. php/revista/article/view/54 
301. Cindy Ng LW, Mackney J, Jenkins S, Hill K. Does exercise training change physical activity in people with COPD? A systematic review and meta-analysis. Chron Respir Dis. 2012;9(1):17-26.

302. Pitta F, Troosters T, Spruit MA, Decramer M, Gosselink R. Activity monitoring for assessment of physical activities in daily life in patients with chronic obstructive pulmonary disease. Arch Phys Med Rehabil. 2005;86(10):1979-85.

303. Prince SA, Adamo KB, Hamel ME, Hardt J, Connor Gorber S, Tremblay M. A comparison of direct versus self-report measures for assessing physical activity in adults: a systematic review. Int J Behav Nutr Phys Act. 2008;5:56.

304. Fitzsimons CF, Baker G, Wright A, Nimmo MA, Ward Thompson C, Lowry R, et al. The «Walking for Wellbeing in the West» randomised controlled trial of a pedometer-based walking programme in combination with physical activity consultation with 12 month follow-up: rationale and study design. BMC Public Health. 2008;8:259.

305. O'Shea SD, Taylor NF, Paratz JD. . . . But watch out for the weather: factors affecting adherence to progressive resistance exercise for persons with COPD. J Cardiopulm Rehabil Prev. j2007;27(3):166-174; quiz $175-176$

306. Tucker P, Gilliland J. The effect of season and weather on physical activity: a systematic review. Public Health. 2007;121(12):909-22.

307. Wewel AR, Gellermann I, Schwertfeger I, Morfeld M, Magnussen H, Jörres RA. Intervention by phone calls raises domiciliary activity and exercise capacity in patients with severe COPD. Respir Med. 2008;102(1):20-6. 


\section{ACRÓNIMOS}

ACSM: American College of Sport Medicine.

AF: Actividad física.

AHA: American Heart Association.

ATP: Adenosín-Trifosfato.

ATS: American Thoracic Society.

BODE: Body Mass Index, Obstrucion, Dyspnea and Exercise Index.

BODEX: Body Mass Index, Obstruction, Dyspnea and Exacerbations Index.

ECA: Ensayo clínicoaleatorizado.

ELTGOL: Espiración lenta total con glotis abierta en decúbito lateral.

EPOC: Enfermedad Pulmonar Obstructiva Crónica.

ERS: European Respiratory Society.

EST: Endurance Shuttle Test (Prueba de Lanzadera a Carga Constante).

FC: Frecuencia cardiaca.

$\mathrm{FEV}_{1}$ : Volumen espiratorio forzado en el primer segundo.

$\mathrm{FEV}_{1}$ (\%): Porcentaje del FEV 1 respecto al valor teórico.

FNP: Facilitación Neuromuscular Propioceptiva.

FVC: Capacidad vital forzada.

FVC (\%): Porcentaje de la FVC respecto al valor teórico.

GesEPOC: Guía española para el manejo de la EPOC.

GOLD: Global Iniciative for Obstructive Lung Disease.

IMC: Índice de masa corporal.

Ipm: Latidos por minuto.

IST: Incremental Shuttle Test (Prueba de Lanzadera Incremental).

MET: Equivalente metabólico de consumo energético.

MCID: Diferencia mínima clínicamente importante.

MMII: Miembros inferiores.

mMRC: Escala Modificada del Medical Research Council.

MMSS: Miembros superiores.

OCD: Oxigenoterapia continua domiciliaria.

OMS: Organización Mundial de la Salud.

PAL: Physical Activity Level.

PEM: Presión espiratoria máxima.

PIM: Presión inspiratoria máxima. 
PRR: Programas d Rehabilitación Respiratoria

RR: Rehabilitación Respiratoria.

$\mathrm{SaO}_{2}$ : Saturación de oxígeno.

SEPAR: Sociedad Española de Neumología y Cirugía Torácica.

SGRQ: St. George's Respiratory Questionaire.

TA: Tensión arterial.

TEF: Técnicas de espiración forzada.

$\mathrm{VO}_{2}$ max: Consumo máximo de oxígeno.

Wmax: Carga máxima de trabajo tolerada.

1RM: Resistencia máxima tolerada en una contracción muscular isotónica concéntrica.

6MWT: Prueba de Marcha de Seis Minutos. 
ANEXOS 


\section{ANEXO I}

\section{CLASIFICACIÓN DE LA EPOC SEGÚN DISTINTAS ESCALAS}

CLASIFICACIÓN GOLD 2001

\begin{tabular}{|c|c|}
\hline Grado & \% FEV $_{\mathbf{1}}$ \\
\hline I - Leve & $\geq 80 \%$ \\
\hline II - Moderado & $50-79 \%$ \\
\hline III - Severo & $35-49 \%$ \\
\hline IV - Muy severo & $<35 \%$ \\
\hline
\end{tabular}

ÍNDICE BODE

\begin{tabular}{|c|c|c|c|c|c|}
\hline & Puntuación* & 0 & $\mathbf{1}$ & $\mathbf{2}$ & $\mathbf{3}$ \\
\hline B & IMC (kg/m²) & $>21$ & $\leq 21$ & & \\
\hline O & FEV $(\%)$ & $\geq 65$ & $50-64$ & $36-49$ & $\leq 35$ \\
\hline D & Disnea (mMRC) & $0-1$ & 2 & 3 & 4 \\
\hline E & 6 6MWT (m) & $\geq 350$ & $250-349$ & $150-249$ & $\leq 149$ \\
\hline
\end{tabular}

IMC: índice de masa corporal

mMRC: escala modificada del Medical Research Council.

6MWT: distancia recorrida en la Prueba de Marcha de Seis Minutos.

(*) La puntuación total del índice BODE se obtiene mediante la suma de las puntuaciones individuales de cada componente, entre 0 y 10 . Las puntuaciones más altas representan un peor pronóstico de la enfermedad. 
ÍNDICE BODEX

\begin{tabular}{|c|c|c|c|c|c|}
\hline & Puntuación* & 0 & 1 & $\mathbf{2}$ & $\mathbf{3}$ \\
\hline B & IMC $\left(\mathrm{kg} / \mathrm{m}^{2}\right)$ & $>21$ & $\leq 21$ & \\
\hline $\mathbf{O}$ & FEV $_{\mathbf{1}}(\%)$ & $\geq 65$ & $50-64$ & $36-49$ & $\leq 35$ \\
\hline D & Disnea $(\mathrm{mMRC})$ & $0-1$ & 2 & 3 & 4 \\
\hline Ex & $\mathbf{N}^{\circ}$ Exacerb/año & 0 & $1-2$ & 3 o más & \\
\hline
\end{tabular}

IMC: índice de masa corporal

mMRC: escala modificada de la MRC

Ex: número de exacerbaciones al año.

(*) La puntuación total del índice BODEX se obtiene mediante la suma de las puntuaciones individuales de cada componente, entre 0 y 9 . Las puntuaciones más altas representan un peor pronóstico de la enfermedad.

CLASIFICACIÓN GOLD (ATUALIZACIÓN DE 2011)

\begin{tabular}{|l|c|c|c|c|}
\hline \multicolumn{1}{|c|}{ Grupo } & GOLD & Exac. & CAT & mMRC \\
\hline A - Bajo riesgo, pocos síntomas & \multirow{2}{*}{ I - II } & \multirow{2}{*}{$0-1$} & $<10$ & $0-1$ \\
\cline { 1 - 3 } B - Bajo riesgo, muchos síntomas & & & $\geq 10$ & $\geq 2$ \\
\hline C - Alto riesgo, pocos síntomas & \multirow{2}{*}{ III - IV* } & \multirow{2}{*}{$\geq 2$} & $<10$ & $0-1$ \\
\cline { 1 - 3 } D - Alto riesgo, muchos síntomas & & & $\geq 10$ & $\geq 2$ \\
\hline
\end{tabular}

GOLD: clasificación según Iniciativa Global Obstructive Lung Disease de 2001.

mMRC: escala modificada del Medical Research Council.

CAT: puntuación del el COPD Assesement Test.

Exac:. número de exacerbaciones al año.

*En la Iniciativa GOLD de 2016 se modifica el nivel de corte para la clasificación de los pacientes en Grado III y IV, siendo Grado III aquellos con $\mathrm{FEV}_{1}$ entre 30-49\% del valor teórico y Grado IV, individuos con $\mathrm{FEV}_{1}<30 \%$. 


\section{ANEXO II}

\section{HOJA INFORMATIVA SOBRE LA REALIZACIÓN DE PROGRAMAS DE REHABILITACIÓN RESPIRATORIA EN LA ESCUELA UNIVERSITARIA DE FISIOTERAPIA DE LA ONCE}

Esta documentación ha sido elaborada conforme a la Ley General de Sanidad 14/1986, de 25 de abril y la Ley 41/2002, de 14 de noviembre, reguladora ésta última de los derechos y obligaciones en materia de información y documentación clínica.

Es importante que lea esta información de forma cuidadosa y completa. Por favor, firme el documento que le presentarán indicando así que lo ha leído y comprende su información. Es importante que hayan sido respondidas todas sus preguntas antes de que firme el documento de consentimiento, que expresa su decisión libre y voluntaria de participación en el estudio y/o tratamiento.

La Fisioterapia es la ciencia que estudia el uso de los agentes físicos con fines terapéuticos. La gran mayoría de los elementos que obran en la naturaleza, como la luz, el calor, el sonido, la electricidad o el movimiento entre otros, pueden ser utilizados con éxito en el tratamiento de los trastornos de la salud. El personal sanitario especializado en esta tarea es el fisioterapeuta. Usted va a ser sometido a un Programa de Rehabilitación Respiratoria (PRR), que específicamente para su tratamiento, incluirá:

- Evaluación funcional respiratoria: exploración física torácica, auscultación pulmonar y pruebas de función pulmonar.

- Evaluación de la tolerancia al ejercicio (pruebas de esfuerzo) y el nivel de actividad física habitual.

- Valoración de la calidad de vida y del sueño a través de cuestionarios específicos.

- Técnicas de Fisioterapia Respiratoria: ejercicios ventilatorios, técnicas dirigidas a la movilización y eliminación de secreciones bronquiales, técnicas de relajación y ejercicios de flexibilización torácica.

- Educación sanitaria enfocada al manejo de la enfermedad, al control de síntomas y al empleo de los dispositivos de administración de fármacos.

- Entrenamiento físico global.

- Evaluación y entrenamiento de los músculos respiratorios.

Los objetivos generales de los PRR son:

- Mejorar la calidad de vida, disminuyendo la sintomatología asociada a la enfermedad.

- Reeducar el patrón ventilatorio, alcanzando una ventilación más eficaz y fisiológica.

- Garantizar una adecuada higiene bronquial.

- Flexibilizar la caja torácica, evitando su rigidez y corrigiendo actitudes posturales anómalas.

- Aumentar la fuerza y resistencia de los músculos respiratorios.

- Incrementar la fuerza y resistencia global, lo que redundará en una mayor tolerancia al esfuerzo. 
Las técnicas de reeducación ventilatoria, así como las de higiene bronquial, flexibilización torácica y la evaluación funcional respiratoria, carecen de riesgo alguno ni efectos adversos a corto o largo plazo, siempre que se respeten las contraindicaciones.

Por otro lado, la realización de las pruebas de esfuerzo y del entrenamiento físico, pueden conducir a sensación de disnea transitoria, fatiga muscular y ligero aumento de la tensión arterial posterior al ejercicio, que revertirá a los pocos minutos. Excepcionalmente pueden producirse complicaciones más severas, como dolorimiento articular y muscular más duradero, y accidentes cardiovasculares, en el caso de que el paciente tenga patología cardíaca previa.

Por su parte, el entrenamiento de músculos respiratorios puede conducir a cefalea y sensación de mareo posterior, puesto que en algunos casos se produce un incremento moderado de la presión intracraneal.

No podrán participar en un PRR aquellos pacientes que presenten hemoptisis, cuadros infecciosos en fase activa o enfermedad cardiovascular descompensada. Estos programas no están contraindicados en pacientes con patología cardiorrespiratoria controlada y estable, puesto que tanto las pruebas de esfuerzo como el entrenamiento físico, se adaptarán a su situación clínica.

Por último, la valoración de la fuerza de los músculos respiratorios, así como su entrenamiento, están contraindicados en pacientes con hipertensión craneal u ocular, alteraciones en el oído medio, cirugía digestiva reciente y riesgo de desarrollar neumotórax. 


\section{DECLARACIÓN DE CONSENTIMIENTO}

D./D. ${ }^{a}$

con DNI. mayor de edad.

D./D. ${ }^{a}$

con DNI.

mayor de edad, en calidad de representante legal o familiar

de D./D. ${ }^{a}$

\section{DECLARO:}

Que he leído la información que me ha sido proporcionada en cuanto al procedimiento, los riesgos y los beneficios de la intervención de Fisioterapia que se me va a realizar (a mí mismo/a o a mi representado/a).

Que he tenido la oportunidad de hablar con

D./D. ${ }^{a}$

y hacer preguntas sobre todos los aspectos de la intervención y me han sido contestadas de forma clara y precisa.

Haber facilitado de manera leal y verdadera los datos sobre el estado físico y salud de mi persona (o de mi representado/a) que pudiera afectar a las intervenciones que se van a realizar.

Que no me encuentro (o mi representado/a) en ninguno de los casos de las contraindicaciones que se me han especificado.

Que con mi firma en este documento, consiento que se me aplique (a mí mismo/a o a mi representado/a) la intervención de Fisioterapia que se me ha explicado de forma suficiente y comprensible.

Que entiendo que tengo el derecho de rehusar parte o todo el tratamiento en cualquier momento, sin tener que dar explicaciones y sin que tenga repercusión alguna.

En Madrid, a ..... de de $20 \ldots . .$. 
Por la presente, otorgo mi consentimiento informado y libre para recibir la intervención de Fisioterapia aquí mencionada.

Leído y Conforme: Firma del paciente o representante.

Nombre y apellidos:

DNI.:

He explicado por completo los detalles relevantes de esta intervención de Fisioterapia al paciente nombrado anteriormente.

Firma del fisioterapeuta responsable:

Nombre y apellidos:

N. ${ }^{\circ}$ de Colegiado: 
Revoco el consentimiento prestado en fecha y no deseo seguir con el tratamiento que doy con esta fecha finalizado.

En Madrid, a ..... de .................. de 20......

Firma del paciente o representante:

Nombre y apellidos:

DNI.:

De acuerdo con lo establecido por la Ley Orgánica 15/1999 de Protección de Datos de Carácter Personal, se le comunica que los datos por usted proporcionados en este documento forman parte de un fichero automatizado y/o manual, cuya única finalidad es la observancia de las obligaciones y derechos nacidos de la firma del mismo. El responsable del fichero es la ORGANIZACIÓN NACIONAL DE CIEGOS ESPAÑOLES (ONCE), con domicilio en la calle Prado $n .^{\circ} 24$ Madrid (28014), en cuya Dirección General podrá ejercitar los derechos de acceso, rectificación, cancelación y oposición que legalmente le asisten. 


\section{ANEXO III \\ CONSENTIMIENTO INFORMADO PARA LA UTILIZACIÓN DE DATOS \\ PERSONALES VINCULADOS A ESTUDIOS DE INVESTIGACIÓN EN LA ESCUELA \\ UNIVERSITARIA DE FISIOTERAPIA DE LA ONCE}

\section{Datos del Centro}

Escuela universitaria de Fisioterapia de la O.N.C.E.

C/ Nuria, 42

28034 - Madrid

Tfno.: 915894500 Fax: 915894498

Email: euf@once.es

Página web: www.once.es/euf

\section{Datos del paciente}

Apellidos y nombre:

Domicilio:

C.P:

Localidad:

Teléfonos:

e-mail:

La Escuela Universitaria de Fisioterapia de la O.N.C.E., adscrita a la Universidad Autónoma de Madrid (U.A.M.), le informa que, de conformidad con la Ley Orgánica 15/1999, de 13 de diciembre, de Protección de Datos de Carácter Personal, los datos personales que Vd. facilite a esta entidad, así como los que se pudieran desarrollar en un futuro, serán incluidos en un fichero del que es responsable la Escuela Universitaria de Fisioterapia de la O.N.C.E., cuya dirección figura en el presente documento.

Por tanto, le pedimos su consentimiento para que la Escuela Universitaria de Fisioterapia de la O.N.C.E. pueda utilizar la información obtenida de las intervenciones realizadas sobre Vd., con fines clínicos, educativos y de investigación, dentro del marco legal y sin revelar sus datos identificativos.

Consiento el uso de la información obtenida de las intervenciones recibidas, con fines clínicos, educativos y de investigación, siempre que no se revele mi identidad.

Vd. podrá ejercitar en cualquier momento los derechos de acceso a sus datos de carácter personal, rectificación, cancelación u oposición al tratamiento de los mismos mediante solicitud 
escrita, acompañada de fotocopia del DNI u otro documento oficial de identificación personal, dirigida a la dirección del centro que figura en este documento.

En Madrid, a ....... de ................... de $20 \ldots \ldots$

Firma del Investigador

Nombre y apellidos:

Leído y Conforme: Firma del paciente.

Nombre y apellidos: 


\section{ANEXO IV}

\section{HOJA DE INFORMACIÓN DEL ESTUDIO:}

\section{EFICACIA DE UN PROGRAMA DE ENTRENAMIENTO AUTOADMINISTRADO E INCREMENTO DEL NIVEL DE ACTIVIDAD GUIADO MEDIANTE PODÓMETRO EN PACIENTES CON EPOC}

La Escuela Universitaria de Fisioterapia de la ONCE (Universidad Autónoma de Madrid), el Servicio de Neumología de la Fundación Jiménez Díaz (Madrid), del Hospital La Paz (Madrid) y del Hospital de La Princesa (Madrid), van a realizar un estudio en el cual está usted invitado a participar.

El estudio tiene como objetivo principal determinar los efectos de dos programas de adhesión al ejercicio físico, sobre los niveles de actividad física, la tolerancia al ejercicio, la calidad de vida, el nivel de disnea basal y el número de exacerbaciones, en pacientes con EPOC estable y perfil de sedentarismo. Podrán participar en el estudio todos aquellos pacientes diagnosticados de EPOC que acudan a consulta en los Servicios de los Centros participantes, y que cumplan una serie de criterios preestablecidos.

Todos los participantes en el proyecto serán asignados a un grupo de estudio mediante un sistema aleatorio, sin posibilidad de ser modificada dicha distribución.

La intervención comenzará con 5 sesiones grupales (3-4 pacientes por grupo) de Fisioterapia Respiratoria de carácter educativo. Las sesiones se realizarán en días consecutivos, y en ellas se le adiestrará en el desarrollo de técnicas de reeducación ventilatoria, maniobras destinadas a la eliminación de secreciones, manejo de los dispositivos de inhalación y cinesiterapia activa libre del tórax. Posteriormente, recibirá uno de los dos programas de adhesión al ejercicio que queremos comparar.

Al inicio del estudio se le harán ciertas mediciones y pruebas sencillas:

a. Medición de la disnea basal (Escala Medical Research Council).

b. Cumplimentación del cuestionario de calidad de vida, St. George's Respiratory Questionaire.

c. Prueba de Lanzadera. Se trata de un test de esfuerzo incremental de marcha estructurado en niveles crecientes de dificultad. En cada nivel deberá caminar a una determinada velocidad en un circuito de $10 \mathrm{~m}$. La velocidad será marcada por señales sonoras e irá aumentando progresivamente. El test finaliza cuando Ud. sea incapaz de mantener la velocidad marcada por la grabación.

d. Prueba de Lanzadera a Carga Constante. Se trata de un test de marcha a carga constante, en el que Ud. deberá caminar a una velocidad que corresponderá al $70 \%$ del nivel de esfuerzo máximo alcanzado en la prueba anterior. Finalizará la prueba cuando no pueda mantener la velocidad marcada.

e. Medición de la actividad física diaria mediante el Cuestionario de Actividad Física Baecke Modificado y del registro podométrico del número de pasos caminados a lo largo de una semana. Para ello, se le hará entrega de un podómetro digital, que tendrá que llevar puesto durante toda la semana. En este tiempo, no deberá realizar ninguna actividad fuera de su rutina cotidiana.

Estas mediciones se repetirán tras diez semanas, a los 3 y a los 12 meses. Además, en el último registro, se le preguntará por el número de exacerbaciones que haya sufrido en esos 12 meses. 
No existen efectos secundarios asociados al desarrollo del programa o de las mediciones que se le van a realizar. Todas las intervenciones serán efectuadas por personal sanitario competente, con lo que los riesgos son escasos. Sólo en el caso de las pruebas de esfuerzo, pueden aparecer algunos efectos adversos, tales como cansancio intenso, dolorimiento muscular o articular posterior por fatiga, sensación de mareo si el ejercicio es muy intenso y disnea posterior, que desaparecen a los pocos minutos. Otras consecuencias del ejercicio físico, que aparecen con muy poca probabilidad son: lesión muscular o articular, episodio de isquemia miocárdica de mayor o menor intensidad y pérdida de conocimiento. Para minimizar estos riesgos, se excluirán del estudio a aquellos pacientes con problemas cardiovasculares y/o del aparato locomotor.

Todos los datos recogidos de su participación en este estudio serán considerados confidenciales, de forma que sólo los investigadores del proyecto conozcan su identidad. Ni su nombre ni cualquier otra seña que le pudiera identificar serán divulgados. Del mismo modo, si abandona el proyecto antes de su finalización, todos los datos recogidos hasta ese momento, serán destruidos.

Si decide participar en el estudio, es requisito imprescindible que ponga la fecha y que firme el documento de consentimiento informado, así como el de cesión de datos que le adjuntamos. Su participación desinteresada y voluntaria en este estudio aportará un gran beneficio al conocimiento científico y permitirá la posibilidad de seguir investigando y desarrollando nuevos programas de tratamiento más eficaces para los pacientes con EPOC.

\section{Gracias por su colaboración.}


DOCUMENTO DE CONSENTIMIENTO INFORMADO PARA EL ESTUDIO:

EFICACIA DE UN PROGRAMA DE ENTRENAMIENTO AUTOADMINISTRADO E INCREMENTO DEL NIVEL DE ACTIVIDAD GUIADO MEDIANTE PODÓMETRO EN PACIENTES CON EPOC

Don/Doña.:

de años de edad, con domicilio en:

y DNI:

\section{DECLARO}

Que D/Dña.

de la Escuela Universitaria de Fisioterapia de la ONCE,

con teléfono de contacto

me ha invitado a participar en este estudio.

Que he leído la hoja de información que se me ha entregado, y que he comprendido las explicaciones que se me han facilitado en un lenguaje claro y sencillo, y que el investigador/a que me ha atendido me ha permitido realizar todas las observaciones y me ha aclarado todas las dudas que le he planteado.

Que entiendo que mi participación es voluntaria, y que el hecho de que no quiera participar en este estudio no producirá ningún trato diferente en cuanto a calidad de asistencia médica o en mi tratamiento.

Que si decido participar, podré cambiar de opinión en cualquier momento y retirarme del estudio en cuanto lo desee, sin necesidad de dar ninguna explicación, y por tanto puedo revocar mi consentimiento que ahora presto.

Por ello, manifiesto que estoy satisfecho/a de la información recibida, he podido formular todas las preguntas que he creído conveniente y me han aclarado todas las dudas planteadas. $Y$ en tales condiciones, presto libremente mi conformidad para participar en el estudio.

Asimismo (marque con una x lo que proceda):

Estoy de acuerdo en el uso de los datos para otras investigaciones: Sí ..... NO .....

Estoy de acuerdo en el uso de los datos para la enseñanza: Sí ..... NO .....

En Madrid, a de de $20 \ldots$.

Fdo.: El/la Investigador/a

Fdo.: El/la Paciente 


\section{REVOCACIÓN}

Don/Doña.:

de ........ años de edad, con domicilio en:

y DNI:

REVOCO el consentimiento prestado en fecha y no deseo proseguir el estudio, que doy con esta fecha por finalizado.

En Madrid, a de de $20 \ldots$.

Fdo.: El/la Investigador/a

Fdo.: El/la Paciente 


\section{CONSENTIMIENTO INFORMADO PARA LA UTILIZACIÓN DE DATOS PERSONALES \\ VINCULADOS AL ESTUDIO:}

\section{EFICACIA DE UN PROGRAMA DE ENTRENAMIENTO AUTOADMINISTRADO E INCREMENTO DEL NIVEL DE ACTIVIDAD GUIADO MEDIANTE PODÓMETRO EN PACIENTES CON EPOC}

\section{Datos del Centro}

Escuela universitaria de Fisioterapia de la O.N.C.E.

C/ Nuria, 42

28034 - Madrid

Tfno.: 915894500 Fax: 915894498

Email:euf@once.es

Página web: www.once.es/euf

\section{Datos del paciente}

Apellidos y nombre:

Domicilio:

C.P:

Localidad:

Teléfonos:

e-mail:

La Escuela Universitaria de Fisioterapia de la O.N.C.E., adscrita a la Universidad Autónoma de Madrid (U.A.M.), le informa que, de conformidad con la Ley Orgánica 15/1999, de 13 de diciembre, de Protección de Datos de Carácter Personal, los datos personales que Vd. facilite a esta entidad al inicio del estudio, así como los que se pudieran desarrollar en un futuro, serán incluidos en un fichero del que es responsable la Escuela Universitaria de Fisioterapia de la O.N.C.E., cuya dirección figura en el presente documento.

Por tanto, le pedimos su consentimiento para que la Escuela Universitaria de Fisioterapia de la O.N.C.E. pueda utilizar la información obtenida de las intervenciones realizadas sobre Vd., con fines clínicos, educativos y de investigación, dentro del marco legal y sin revelar sus datos identificativos.

Asimismo, le pedimos su consentimiento para poder usar sus datos personales en el registro de su evolución y el seguimiento del proceso iniciado, así como para que reciba de esta entidad informaciones relativas a nuestros servicios clínicos, por cualquier medio, incluido el correo electrónico o medio electrónico equivalente, aún después de finalizada su participación en el estudio. Si Vd. consiente el uso de sus datos con las finalidades indicadas, marque una $x$ en el apartado correspondiente. 
Consiento el uso de la información obtenida de las intervenciones recibidas, con fines clínicos, educativos y de investigación, siempre que no se revele mi identidad.

Consiento el uso de mis datos con finalidades de información indicadas anteriormente, incluido el uso de medios electrónicos para la recepción de las mismas.

Vd. podrá ejercitar en cualquier momento los derechos de acceso a sus datos de carácter personal, rectificación, cancelación u oposición al tratamiento de los mismos mediante solicitud escrita, acompañada de fotocopia del DNI u otro documento oficial de identificación personal, dirigida a la dirección del centro que figura en este documento.

En Madrid, a

de de $20 \ldots$.

Firma del Investigador

Nombre y apellidos:

Leído y Conforme: Firma del paciente.

Nombre y apellidos: 


\section{ANEXO V \\ REGISTRO DE ACTIVIDAD (ESTUDIO 2) \\ INSTRUCCIONES PARA UTILIZAR ESTE DIARIO}

Los investigadores de este estudio le proponen que use este documento para conseguir un buen control de la actividad llevada a cabo por usted, durante las 8 semanas que dura el programa (una hoja por cada semana).

En esta hoja se detallan una serie de instrucciones que le facilitarán el registro de todos aquellos datos que tendrá que proporcionar a las personas que cada semana contactarán con usted por teléfono para hacer el seguimiento.

1. Anote en la primera fila de la tabla y en su casilla correspondiente, el $n^{\circ}$ de pasos que realiza cada día de la semana. Recuerde que para el control de sus pasos, debe llevar consigo el podómetro que le han proporcionado, durante todo el día.

2. Sume el $n^{\circ}$ de pasos que haya dado durante toda la semana, menos los realizados durante las caminatas del programa de marcha y refleje el total en la casilla " $\mathrm{N}^{\circ}$ de pasos semanales".

3. Compruebe, comparando esta cantidad con la que aparece en la casilla "Pasos semanales esperados", si ha conseguido el objetivo marcado para esta semana.

4. Marque con una $X$, los cinco días de la semana en los que ha llevado a cabo la caminata del programa de marcha, en las casillas de la segunda fila reservadas para ello.

5. El tiempo de caminata y el número de pasos a alcanzar cada día en el programa de marcha, lo tiene indicado en la parte superior derecha de cada hoja. Recuerde que el número de pasos corresponde al total de los ciclos del programa que ha de realizar cada día y que estos ciclos puede efectuarlos en cualquier momento del día, aunque preferiblemente, evitando las horas posteriores a las ingestas alimentarias, así como las de más calor o frío del día.

6. Anote el $n^{\circ}$ total de pasos que haya realizado durante la caminata del programa de marcha en las casillas correspondientes de la tercera fila de la tabla y compare con el $n^{\circ}$ de pasos que debe alcanzar para este programa.

7. Indique, en la cuarta y quinta fila, el nivel de dificultad respiratoria (disnea) que presente antes de realizar la actividad del programa de marcha y la que tenga al terminar éste. Para ello, utilice la escala de Borg que tiene a la derecha de la tabla, señalando el nivel con el $\mathrm{n}^{\circ}$ que coincida con lo que sienta. 
8. Apunte, en la sexta y séptima fila, la sensación de fatiga que tenga en los miembros inferiores antes y después del programa de marcha. Utilice también la escala de Borg.

9. Analice los datos y conteste la pregunta que se le plantea bajo la tabla, indicando si ha cumplido con el $n^{\circ}$ de pasos que se esperaba tanto en la actividad diaria como en el programa de marcha. Si no lo ha conseguido, señale cual es la razón.

La importancia de cumplimentar todos los días este diario es conseguir, de una forma sencilla, recopilar todos aquellos datos que le van a permitir que usted controle la evolución de su programa de marcha y compruebe cómo influye esto en el aumento de su actividad diaria. De igual forma, en estas hojas está recogida toda aquella información que se le va a solicitar semanalmente, para ver su desarrollo y para marcar el nuevo objetivo a conseguir en la semana posterior, que usted mismo anotará en la casilla de "Pasos semanales esperados" de la hoja de la semana siguiente.

Si tiene cualquier duda acerca del desarrollo del programa o de la cumplimentación de este diario, póngase en contacto con nosotros en el teléfono 915894500. 


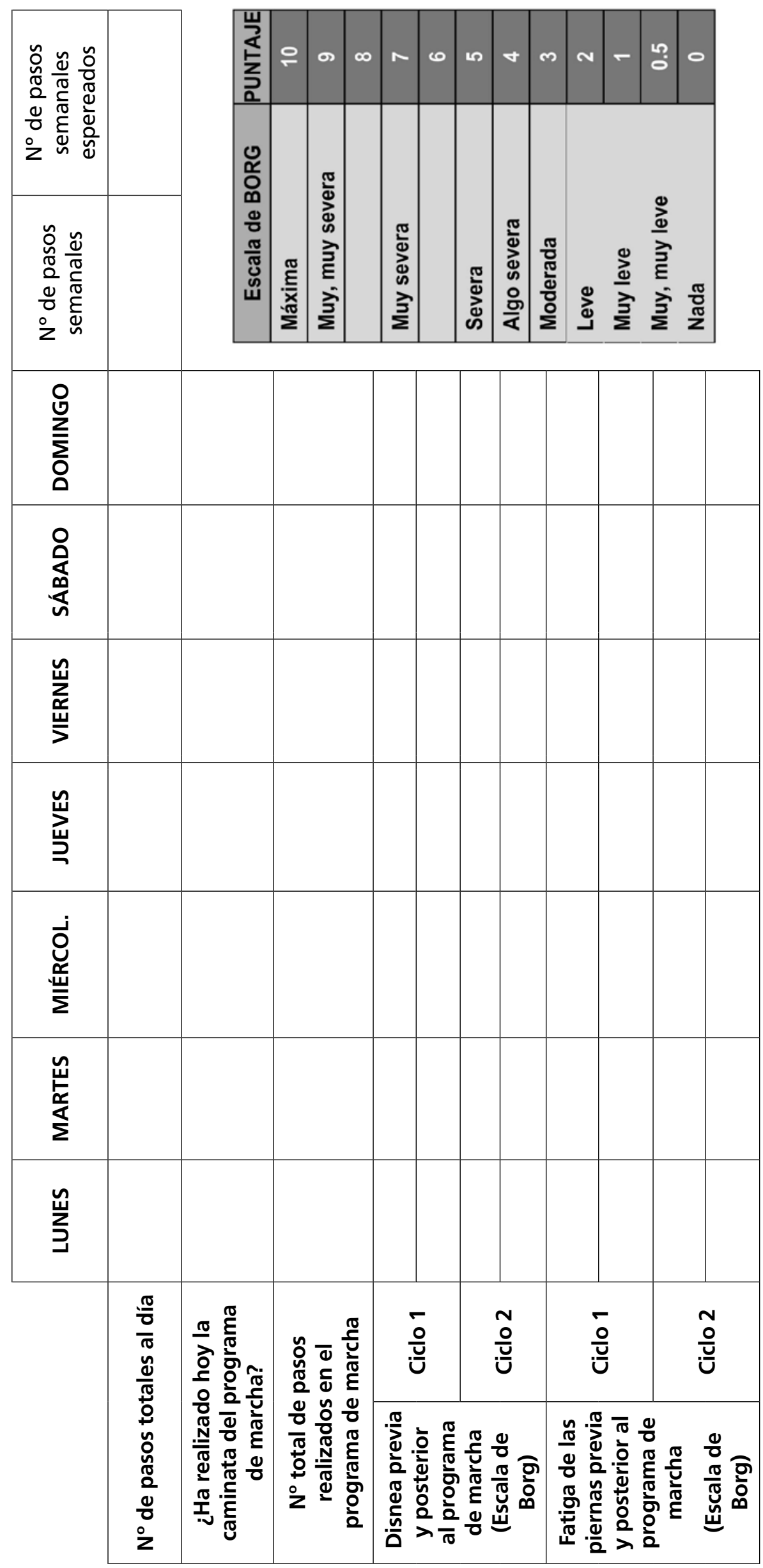

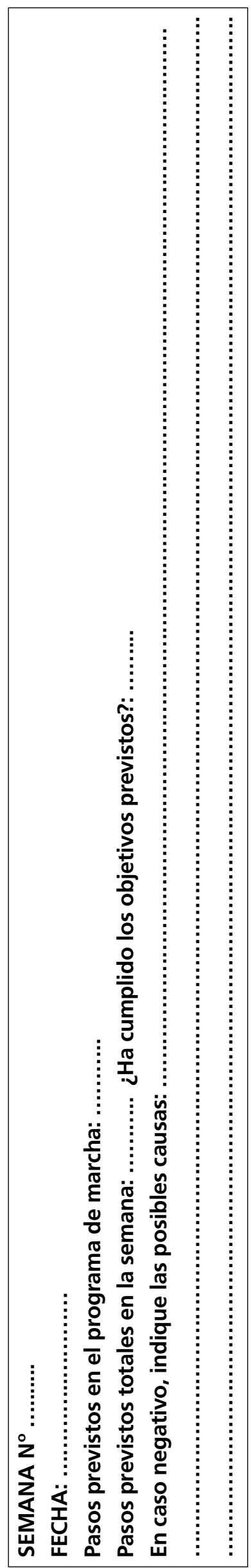




\section{ANEXO VI}

HOJA DE RECOGIDA DE DATOS - ENTREVISTA INICIAL (ESTUDIO 2)

Fecha:

Datos de identificación.

- No Identificación:

- Apellidos:

- Nombre:

- Domicilio:

- Localidad:

- C.P.:

- Teléfonos de contacto:

Datos sociodemográficos y clínicos

\begin{tabular}{|c|c|c|}
\hline \multicolumn{3}{|l|}{ Sexo } \\
\hline \multicolumn{3}{|c|}{ Fecha de nacimiento } \\
\hline \multicolumn{3}{|l|}{ Edad } \\
\hline \multicolumn{3}{|l|}{ Peso } \\
\hline \multicolumn{3}{|l|}{ Altura } \\
\hline \multicolumn{3}{|l|}{ IMC } \\
\hline \multicolumn{3}{|c|}{ Fecha de diagnóstico } \\
\hline $\mathrm{FEV}_{1}$ & $\% \mathrm{FEV}_{1}$ & \\
\hline FVC & $\% F V C$ & \\
\hline $\mathrm{FEV}_{1} / \mathrm{FVC}$ & Clasifica & OLD: \\
\hline
\end{tabular}




\section{Historia tabáquica}

\begin{tabular}{|c|c|c|}
\hline ¿Fumador activo? & Sí ..... & No .... \\
\hline ¿Fumador antiguo? & Sí ..... & No .... \\
\hline \multicolumn{3}{|l|}{ Edad a la que comenzó a fumar } \\
\hline \multicolumn{3}{|l|}{ Edad a la que dejó de fumar } \\
\hline \multirow{8}{*}{$\mathrm{N}^{\circ}$ cigarrillos/día según períodos } & De $10-20$ años & \\
\hline & De $20-30$ años & \\
\hline & De 30-40 años & \\
\hline & De 40-50 años & \\
\hline & De 50-60 años & \\
\hline & De 60-70 años & \\
\hline & De $70-80$ años & \\
\hline & De 80-90 años & \\
\hline Balance paquetes/año & & \\
\hline
\end{tabular}

\section{Comorbilidades - Charlson Index}

Sida

Enfermedad Cerebrovascular EPOC

Insuficiencia Cardiaca Congestiva Enf. Tejido Conectivo

Demencia

Hemiplejia

Leucemia

Linfoma

Infarto de Miocardio

Enf. Vascular periférica

Ulcus péptico

Diabetes

Enf. Hepática leve

Enf. Hepática leve moderada

Enf Renal (mod-sev)

Cáncer (últimos 5 años)

Diabetes con daño orgánico

Metástasis

\section{Si detalles}


Sistemas de soporte ventilatorio

\begin{tabular}{|c|c|}
\hline Sí ....... No ...... & $\mathrm{O}_{2}$ ambulatorio Sí ..... No ...... \\
\hline Sistema de administración & $\begin{array}{l}\text { Depósito gas ....... } \\
\text { Concentrador ........ } \\
\text { Oxígeno líquido ...... }\end{array}$ \\
\hline Horas/día ......... & Dosis ............... \\
\hline
\end{tabular}

Prueba de Marcha de Seis Minutos

\begin{tabular}{|l|l|l|}
\hline \multicolumn{1}{|c|}{ Parámetros de control } & Inicial & Final \\
\hline Grado de disnea (Esc. Borg) & & \\
\hline Fatiga en M.M.I.I. (Esc. Borg) & & \\
\hline Frecuencia cardiaca & & \\
\hline Saturación de oxígeno & & \\
\hline Presión arterial & & \\
\hline
\end{tabular}

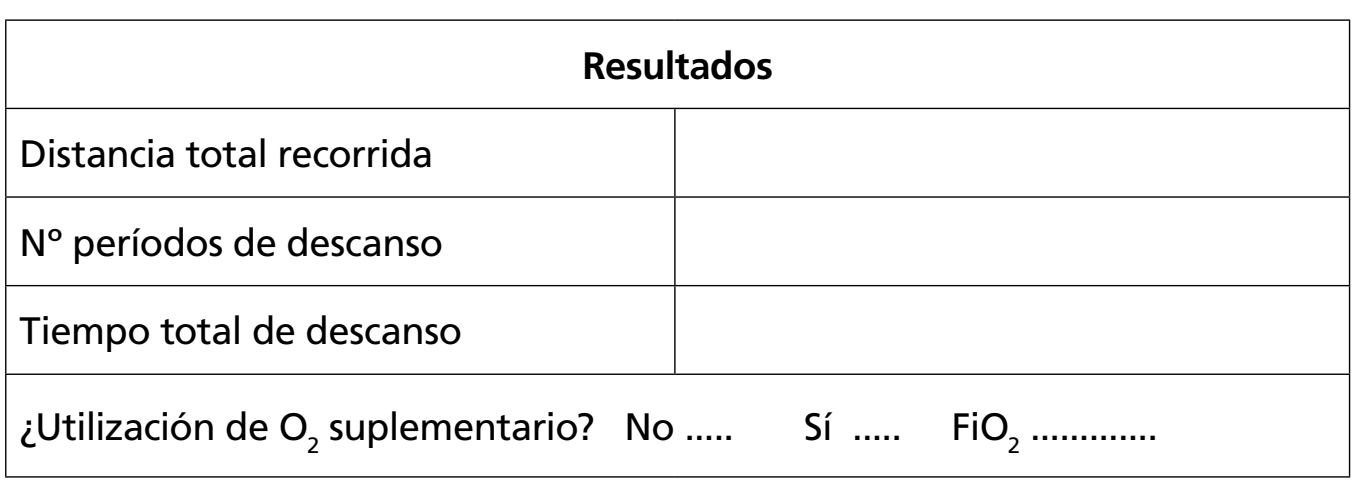

Observaciones: 
ANEXO VII

HOJA DE RECOGIDA DE VARIABLES (ESTUDIO 2)

Nºntificación:

Nombre:

\section{REGISTRO PRE-INTERVENCIÓN}

Fecha:

$\mathrm{N}^{\mathrm{a}}$ exacerbaciones en los 12 meses anteriores:

Disnea basal (Escala mMRC):

Puntuación SGRQ:

\begin{tabular}{|c|c|c|c|}
\hline $\begin{array}{c}\text { Dimensión 1 } \\
\text { Síntomas }\end{array}$ & $\begin{array}{c}\text { Dimensión 2 } \\
\text { Actividad }\end{array}$ & $\begin{array}{c}\text { Dimensión 3 } \\
\text { Impacto }\end{array}$ & TOTAL \\
\hline & & & \\
& & & \\
\hline
\end{tabular}

Puntuación Cuestionario Baecke Modificado:

\begin{tabular}{|c|c|c|c|}
\hline $\begin{array}{c}\text { Dimensión 1 } \\
\text { Actividades de casa }\end{array}$ & $\begin{array}{c}\text { Dimensión 2 } \\
\text { Actividades de ocio }\end{array}$ & $\begin{array}{c}\text { Dimensión 3 } \\
\text { Actividades deportivas }\end{array}$ & TOTAL \\
\hline & & & \\
\hline
\end{tabular}

$\mathrm{N}^{\circ}$ pasos registrados en 7 días:

Test de Shuttle Incremental:

\begin{tabular}{|l|l|l|}
\hline \multicolumn{1}{|c|}{ Parámetros de control } & Inicial & Final \\
\hline Grado de disnea (Esc. Borg) & & \\
\hline Fatiga en M.M.I.I. (Esc. Borg) & & \\
\hline Frecuencia cardiaca & & \\
\hline Saturación de oxígeno & & \\
\hline Presión arterial & & \\
\hline
\end{tabular}




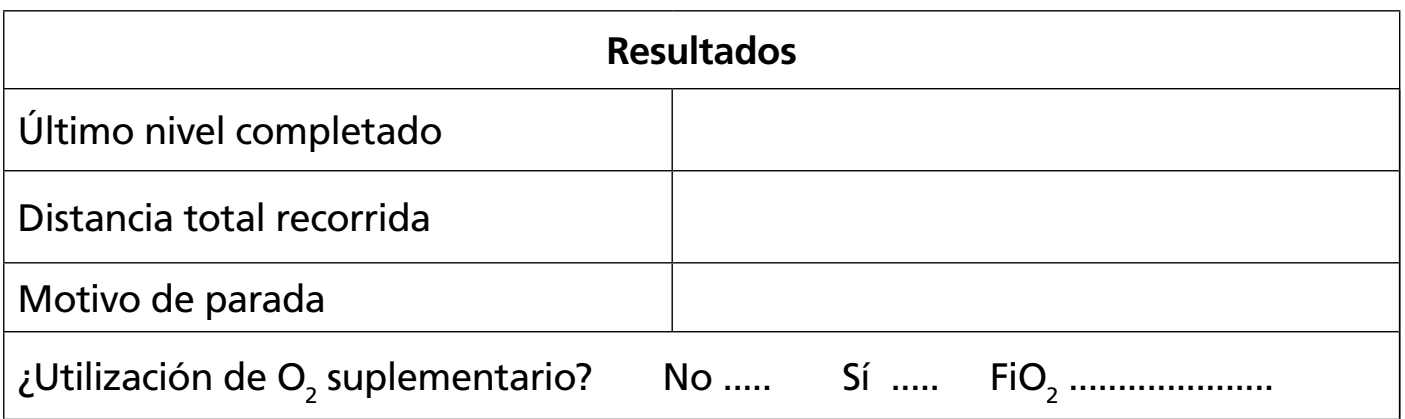

Endurance Shuttle Test:

\begin{tabular}{|l|l|l|}
\hline \multicolumn{1}{|c|}{ Parámetros de control } & Inicial & Final \\
\hline Grado de disnea (Esc. Borg) & & \\
\hline Fatiga en M.M.I.I. (Esc. Borg) & & \\
\hline Frecuencia cardiaca & & \\
\hline Saturación de oxígeno & & \\
\hline Presión arterial & & \\
\hline
\end{tabular}

\begin{tabular}{|c|c|c|c|}
\hline \multicolumn{4}{|c|}{ Resultados } \\
\hline Velocidad de marcha & & & \\
\hline ¿Utilización de $\mathrm{O}_{2}$ suplementario? & No ..... & Sí ...... & $\mathrm{FiO}_{2} \ldots \ldots \ldots \ldots \ldots \ldots \ldots$ \\
\hline \multicolumn{4}{|l|}{ Motivo de parada } \\
\hline Tiempo total caminado ..................... & \multicolumn{3}{|c|}{ Distancia total recorrida.................... } \\
\hline
\end{tabular}

Observaciones: 
REGISTRO 1 POST-INTERVENCIÓN:

Fecha:

Disnea basal (Escala mMRC):

Puntuación SGRQ:

\begin{tabular}{|c|c|c|c|}
\hline $\begin{array}{c}\text { Dimensión 1 } \\
\text { Síntomas }\end{array}$ & $\begin{array}{c}\text { Dimensión 2 } \\
\text { Actividad }\end{array}$ & $\begin{array}{c}\text { Dimensión 3 } \\
\text { Impacto }\end{array}$ & TOTAL \\
\hline & & & \\
& & & \\
\hline
\end{tabular}

Puntuación Cuestionario Baecke Modificado:

\begin{tabular}{|c|c|c|c|}
\hline Dimensión 1 & Dimensión 2 & Dimensión 3 & TOTAL \\
Actividades de casa & Actividades de ocio & Actividades deportivas & . \\
\hline & & & \\
\hline
\end{tabular}

$\mathrm{N}^{\circ}$ pasos registrados en 7 días:

Endurance Shuttle Test:

\begin{tabular}{|l|l|l|}
\hline \multicolumn{1}{|c|}{ Parámetros de control } & Inicial & Final \\
\hline Grado de disnea (Esc. Borg) & & \\
\hline Fatiga en M.M.I.I. (Esc. Borg) & & \\
\hline Frecuencia cardiaca & & \\
\hline Saturación de oxígeno & & \\
\hline Presión arterial & & \\
\hline
\end{tabular}

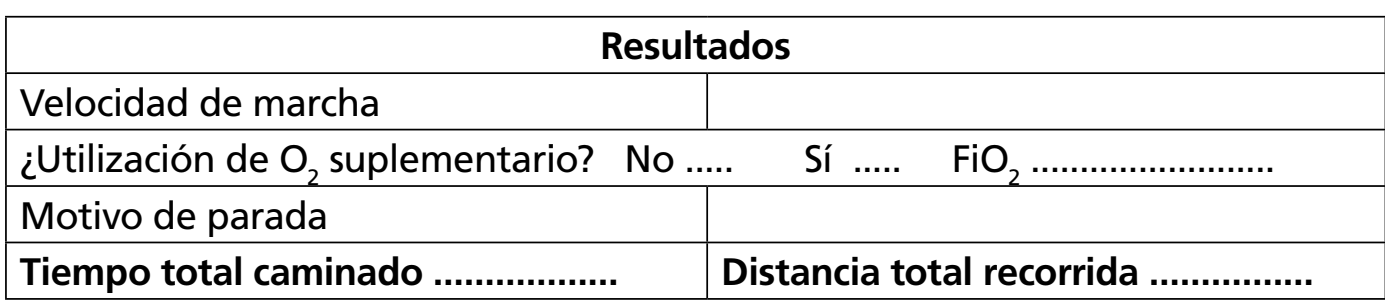

Observaciones: 
REGISTRO 2 POST-INTERVENCIÓN:

Fecha:

Disnea basal (Escala mMRC):

Puntuación SGRQ:

\begin{tabular}{|c|c|c|c|}
\hline $\begin{array}{c}\text { Dimensión 1 } \\
\text { Síntomas }\end{array}$ & $\begin{array}{c}\text { Dimensión 2 } \\
\text { Actividad }\end{array}$ & $\begin{array}{c}\text { Dimensión 3 } \\
\text { Impacto }\end{array}$ & TOTAL \\
\hline & & & \\
& & & \\
\hline
\end{tabular}

Puntuación Cuestionario Baecke Modificado:

\begin{tabular}{|c|c|c|c|}
\hline $\begin{array}{c}\text { Dimensión 1 } \\
\text { Actividades de casa }\end{array}$ & Dimensión 2 & Dimensión 3 & TOTAL \\
\hline & Actividades de ocio & Actividades deportivas & \\
& & & \\
\hline
\end{tabular}

$\mathbf{N}^{\circ}$ pasos registrados en 7 días:

Endurance Shuttle Test:

\begin{tabular}{|l|l|l|}
\hline \multicolumn{1}{|c|}{ Parámetros de control } & Inicial & Final \\
\hline Grado de disnea (Esc. Borg) & & \\
\hline Fatiga en M.M.I.I. (Esc. Borg) & & \\
\hline Frecuencia cardiaca & & \\
\hline Saturación de oxígeno & & \\
\hline Presión arterial & & \\
\hline
\end{tabular}

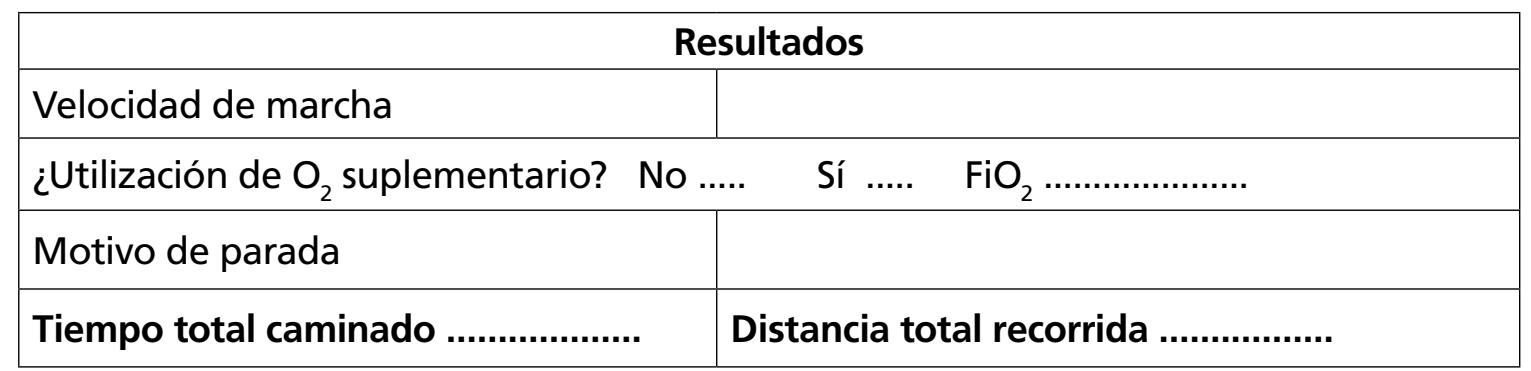

Observaciones: 


\section{REGISTRO 3 POST-INTERVENCIÓN:}

Fecha:

$\mathbf{N}^{\circ}$ exacerbaciones desde el registro 1 post-intervención:

Disnea basal (Escala mMRC):

Puntuación SGRQ:

\begin{tabular}{|c|c|c|c|}
\hline $\begin{array}{c}\text { Dimensión 1 } \\
\text { Síntomas }\end{array}$ & $\begin{array}{c}\text { Dimensión 2 } \\
\text { Actividad }\end{array}$ & $\begin{array}{c}\text { Dimensión 3 } \\
\text { Impacto }\end{array}$ & TOTAL \\
\hline & & & \\
\hline
\end{tabular}

Puntuación Cuestionario Baecke Modificado:

\begin{tabular}{|c|c|c|c|}
\hline $\begin{array}{c}\text { Dimensión 1 } \\
\text { Actividades de casa }\end{array}$ & $\begin{array}{c}\text { Dimensión 2 } \\
\text { Actividades de ocio }\end{array}$ & $\begin{array}{c}\text { Dimensión 3 } \\
\text { Actividades deportivas }\end{array}$ & TOTAL \\
\hline & & & \\
\hline
\end{tabular}

$N^{\circ}$ pasos registrados en 7 días:

Endurance Shuttle Test:

\begin{tabular}{|l|l|l|}
\hline \multicolumn{1}{|c|}{ Parámetros de control } & Inicial & Final \\
\hline Grado de disnea (Esc. Borg) & & \\
\hline Fatiga en M.M.I.I. (Esc. Borg) & & \\
\hline Frecuencia cardiaca & & \\
\hline Saturación de oxígeno & & \\
\hline Presión arterial & & \\
\hline
\end{tabular}

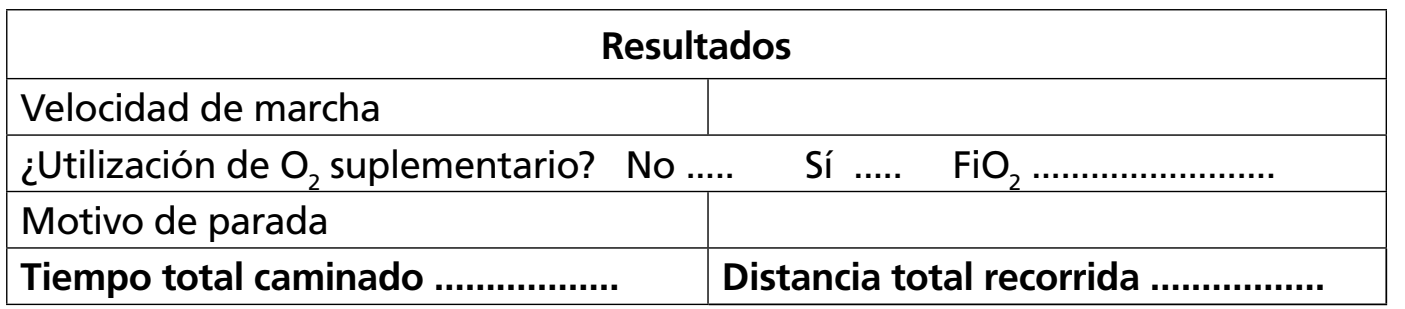

Observaciones: 